\title{
Small fibers, big troubles : diagnosis and implications of small fiber neuropathy
}

Citation for published version (APA):

Bakkers, M. (2015). Small fibers, big troubles : diagnosis and implications of small fiber neuropathy. [Doctoral Thesis, Maastricht University]. Datawyse / Universitaire Pers Maastricht. https://doi.org/10.26481/dis.20150417mb

Document status and date:

Published: 01/01/2015

DOI:

10.26481/dis.20150417mb

Document Version:

Publisher's PDF, also known as Version of record

\section{Please check the document version of this publication:}

- A submitted manuscript is the version of the article upon submission and before peer-review. There can be important differences between the submitted version and the official published version of record.

People interested in the research are advised to contact the author for the final version of the publication, or visit the DOI to the publisher's website.

- The final author version and the galley proof are versions of the publication after peer review.

- The final published version features the final layout of the paper including the volume, issue and page numbers.

Link to publication

\footnotetext{
General rights rights.

- You may freely distribute the URL identifying the publication in the public portal. please follow below link for the End User Agreement:

www.umlib.nl/taverne-license

Take down policy

If you believe that this document breaches copyright please contact us at:

repository@maastrichtuniversity.nl

providing details and we will investigate your claim.
}

Copyright and moral rights for the publications made accessible in the public portal are retained by the authors and/or other copyright owners and it is a condition of accessing publications that users recognise and abide by the legal requirements associated with these

- Users may download and print one copy of any publication from the public portal for the purpose of private study or research.

- You may not further distribute the material or use it for any profit-making activity or commercial gain

If the publication is distributed under the terms of Article $25 \mathrm{fa}$ of the Dutch Copyright Act, indicated by the "Taverne" license above, 


\section{Small fibers, big troubles}

Diagnosis and implications of small fiber neuropathy

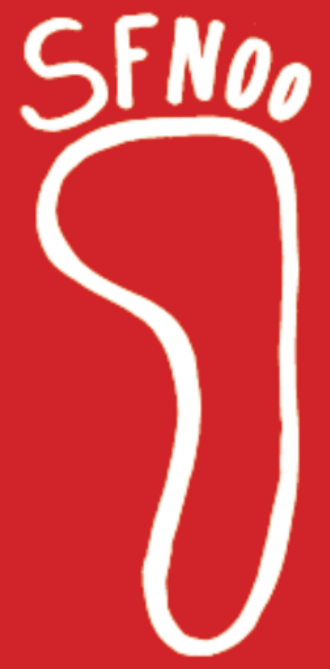

Mayienne Bakkers 
The studies described in this thesis were performed at the Department of Neurology, Maastricht, the Netherlands.

This research project was financially supported by grants from the 'Profileringsfonds azM'.

(c) M. Bakkers, 2015

ISBN 978-94-6159-422-8

Lay-out Jos Hendrix

Printing Universitaire Pers Maastricht 


\title{
Small fibers, big troubles
}

\section{Diagnosis and implications \\ of small fiber neuropathy}

\author{
Proefschrift \\ ter verkrijging van de graad van doctor aan de \\ Universiteit Maastricht \\ op gezag van de Rector Magnificus \\ Prof. dr. L.L.G. Soete \\ volgens besluit van het College van Decanen, \\ in het openbaar te verdedigen op \\ vrijdag 17 april 2015 om 12.00 uur \\ door \\ Mayienne Bakkers
}

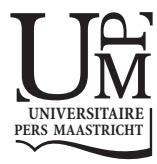




\section{Promotiecommissie}

\section{Promotores:}

Prof. dr. C.G. Faber

Prof. dr. M. De Baets

\section{Co-promotor:}

Dr. I.S.J. Merkies (Spaarne Ziekenhuis Hoofddorp)

\section{Beoordelingscommissie:}

Prof. dr. C.E.M. de Die-Smulders (voorzitter)

Prof. dr. A. zur Hausen

Prof. dr. E.A. Joosten

Dr. A. van der Kooi (Academisch Medisch Centrum)

Dr. B. Kusters (Radboud universitair medisch centrum) 
Aan mijn patiënten, opdat al hun inspanningen hen uiteindelijk verder helpen. 



\section{TABLE OF CONTENTS}

Glossary

Part I General introduction

Chapter 1.1 Clinical aspects of small fiber neuropathy

Chapter 1.2 Outline of the thesis

Part II Diagnosis of small fiber neuropathy 23

Chapter 2.1 Intraepidermal nerve fiber density and its application in sarcoidosis

Chapter 2.2 Intraepidermal nerve fiber density at the distal leg; a worldwide normative reference study

Chapter 3.1 Temperature threshold testing, a systematic review

Chapter 3.2 Optimizing temperature threshold testing in small fiber neuropathy

Chapter 4 Contact heat evoked potentials: Normal values and applicability in small fiber neuropathy

Part III Clinical and clinimetric aspects of small fiber neuropathy

Chapter 5 Incidence and prevalence of small fiber neuropathy:

a survey in the Netherlands

Chapter 6 Pain and autonomic dysfunction in patients with sarcoidosis and small fiber neuropathy

Chapter 7 Small fibers, large impact: quality of life in small fiber neuropathy

Part IV Summary, general discussion and future perspectives

Chapter 8.1 Summary and general discussion

Chapter 8.2 Future perspectives

Chapter 9.1 Nederlandse samenvatting

Chapter 9.2 Toekomst perspectieven

Chapter 10 Valorization

Dankwoord 


\section{Glossary}

$\begin{array}{ll}\text { AUC } & \text { Area Under the Curve } \\ \text { CHEP } & \text { Contact Heat Evoked Potential } \\ \text { CIDP } & \text { Chronic Inflammatory Demyelinating Polyneuropathy } \\ \text { DM } & \text { Diabetes Mellitus } \\ \text { DFNS } & \text { Deutschen Forschungsverbund Neuropathischer Schmerz = German Research } \\ & \text { Network on Neuropathic Pain } \\ \text { GBS } & \text { Guillain-Barré syndrome } \\ \text { IENFD } & \text { Intraepidermal nerve fiber density } \\ \text { LEP } & \text { Laser Evoked Potential } \\ \text { MUMC } & \text { Maastricht University Medical Center } \\ \text { NS } & \text { Not significant } \\ \text { PNQOL97 } & \text { Vickrey Peripheral Neuropathy Quality-of-Life Instrument-97 } \\ \text { QST } & \text { Quantitative Sensory Testing } \\ \text { ROC } & \text { Receiver-Operator Characteristic } \\ \text { SFN } & \text { Small Fiber Neuropathy } \\ \text { SIQ } & \text { Symptoms Inventory Questionnaire } \\ \text { TTT } & \text { Temperature Threshold Testing }\end{array}$




PART I

GENERAL INTRODUCTION 


\section{Chapter 1.1}

\section{CLINICAL ASPECTS OF SMALL FIBER NEUROPATHY}

\section{Symptoms and signs}

Small fiber neuropathy refers to a subtype of polyneuropathies in which (only or predominantly) small nerve fibers are affected: thinly myelinated A-delta fibers (cutaneous cold perception and nociception), unmyelinated C fibers (thermoperception and both chemical, pressure and heat nociception), and thinly myelinated preganglionic and unmyelinated postganglionic autonomic fibers. Patients typically complain of burning, lancinating pain and may have decreased temperature or pinprick sensation as the only sign at examination. Autonomic symptoms can occur, such as dry eyes or mouth, orthostatic intolerance, bowel disturbances (constipation, diarrhea, gastroparesis, cramps), micturation disturbances, sweat changes (hyper- and hypohydrosis), accommodation problems, impotence, ejaculation problems or diminished lubrication, hot flashes, and cardiac palpitations. ${ }^{1-3}$ A symmetrical distal neuropathy seems more prevalent, but small fiber neuropathy may be non-length dependent.4-6.

\section{Diagnosis}

No gold standard exists for the diagnosis of small fiber neuropathy. The combination of typical symptoms, signs, and absence of large fiber involvement is required. Therefore, nerve conduction studies (large fiber function) should be normal, while tests showing small nerve fiber deficit may be abnormal..

Temperature and pain sensation is relayed by small nerve fibers, and several devices and protocols have been developed for temperature threshold testing and (heat) pain detection.7. 8 However, though quantitative measurement is possible, the test result is still considered subjective. ${ }^{9}$ Registration of brain potentials, evoked by laser or thermofoil heat pulses, provides objective results. ${ }^{10}$

Various function tests for the autonomic nervous system exist, examining pupillomotor, cardiac, vasomotor, sudomotor, uro-genital and gastro-intestinal function. ${ }^{1 "}$ However, sensitivity may be low, as has been demonstrated in the past.12-14 
Historically, sural nerve biopsy was necessary to examine (both large and small) peripheral nerve fibers. An invasive test one can only perform twice in one patient, usually resulting in a permanent sensory deficit. With the ongoing development of immunohistochemical techniques, small nerve fiber endings in epidermal layers of the skin and other organs can now be visualized in small tissue biopsies ${ }^{15}$ (Figure 1). Determination of intraepidermal nerve fiber density (IENFD) is considered a reliable technique to assess loss and regeneration of small nerve fibers and is considered the best surrogate marker for the diagnosis SFN. Guidelines have been published on the use of skin biopsies in the diagnosis of SFN. ${ }^{16}$

Figure 1. Skin biopsy.

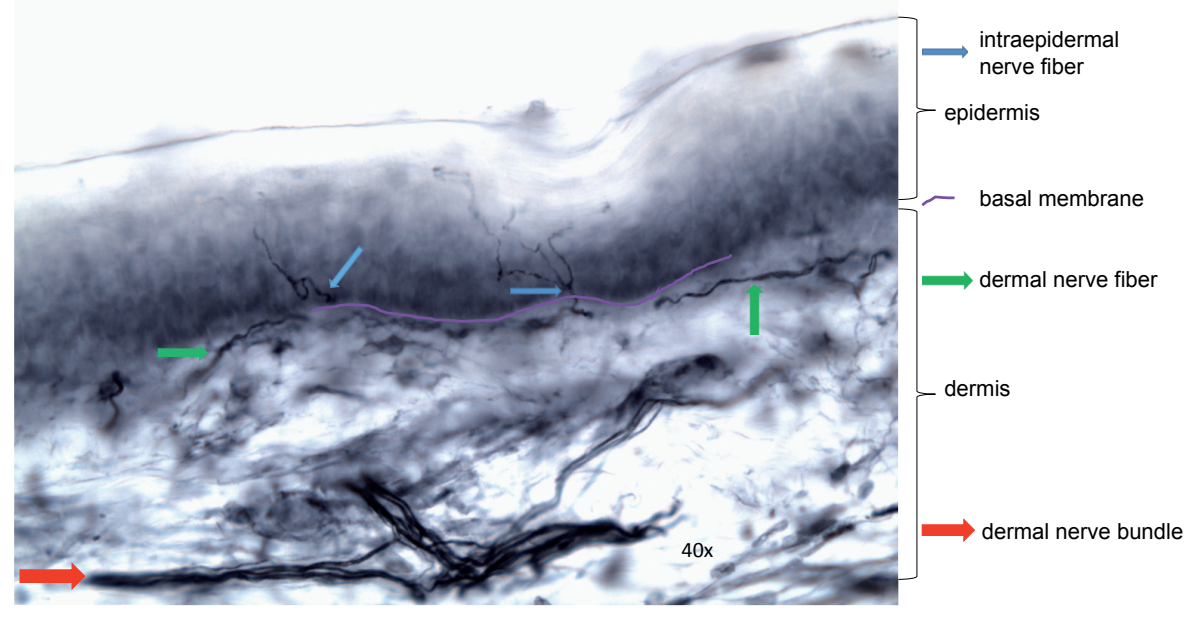

A 3mm punch biopsy of the distal leg, immunostained with PGP 9.5.

For patients with Diabetes Mellitus, a definition of SFN has been proposed, emphasizing clinical features and additional investigations:

- Possible SFN: length-dependent symptoms and/or clinical signs (pinprick and thermal sensory loss and/or hyperalgesia).

- Probable SFN: length-dependent symptoms, clinical signs of small fiber damage and normal nerve conduction studies of sural nerves.

- Definite SFN: length-dependent symptoms, clinical signs of small fiber damage, normal nerve sural nerve conduction studies, and altered intra-epidermal nerve fiber density at the ankle and/or abnormal quantitative sensory testing of thermal thresholds at the foot. ${ }^{17}$

Others strongly recommend using these criteria in SFN of any cause, irrespective of whether the neuropathy is length- or non-length dependent. ${ }^{18,19}$ 


\section{Causes}

SFN is associated with various disorders, such as metabolic, infectious, inflammatory and genetic diseases. Table 1 provides an overview of SFN related disorders. Etiological diagnosis is important in patients with SFN, since underlying causes may need treatment. However, despite a thorough workup, the proportion of patients with idiopathic or cryptogenic SFN remains substantial, ranging from 24\% up to 93\%., 20, 21 In patients with diabetes mellitus, a disease with increasing incidence and prevalence, ${ }^{22}$ an estimated $16-20 \%$ suffers from a painful neuropathy. ${ }^{23,24}$ This is reported to be largely attributed to small fiber involvement. ${ }^{25}$

Voltage gated sodium channels play a cardinal role in neuronal action potentials, ${ }^{26}$ and several syndromes have been described in genetic Nav 1.7 abnormalities. ${ }^{27,}{ }^{28}$ In a recent study, gain-of-function SCN9A gene mutations have been described in SFN patients. ${ }^{29}$ Recently, also mutations in Nav1.8 and Nav1.9 have been found in SFN. ${ }^{30-32}$

Table 1 Conditions associated with small fiber neuropathy.

\begin{tabular}{|c|c|c|c|}
\hline & Pure SFN & $\begin{array}{l}\text { Progression to mixed } \\
\text { polyneuropathy }\end{array}$ & $\begin{array}{l}\text { Reduced IENFD, no } \\
\text { clear SFN }\end{array}$ \\
\hline Metabolic & $\begin{array}{l}\text { Impaired glucose tolerance }{ }^{33,34} \\
\text { Rapid glucose control } \\
\text { Hyperlipidemia }{ }^{36} \text { Hypothyroidism }{ }^{37}\end{array}$ & $\begin{array}{l}\text { Diabetes }{ }^{17,25} \\
\text { Chronic kidney disease }^{38}\end{array}$ & \\
\hline $\begin{array}{l}\text { Immune } \\
\text { mediated }\end{array}$ & $\begin{array}{c}\text { Sarcoidosis }{ }^{39} \\
\text { Sjögrens' syndrome }{ }^{40} \\
\text { Coeliac disease }{ }^{41} \text { Inflammatory } \\
\text { bowel diseases }{ }^{42} \\
\text { Paraneoplastic neuropathy }{ }^{43}\end{array}$ & $\begin{array}{c}\text { Amyloidosis }{ }^{44} \\
\text { Vasculitis }^{45} \\
\text { Systemic lupus erythematodes } \\
46 \\
\text { Guillain-Barré syndrome }{ }^{47}\end{array}$ & \\
\hline Infectious & $\begin{array}{l}\text { Leprosy }^{48} \\
\text { Epstein-Barr virus }\end{array}$ & $\begin{array}{c}\text { HIV }^{50} \\
\text { Hepatitis } C^{51} \\
\text { Lyme neuroborreliosis }^{52}\end{array}$ & \\
\hline Toxic & $\begin{array}{l}\text { Antiretroviral drugs }{ }^{53}, \text { Bortezomib } \\
\text { Metronidazole } \\
\text { Flecainide } \\
\text { Alcohol abuse }^{56} \text { Nitrofurantoin }^{57}\end{array}$ & Hypervitaminosis B6 ${ }^{60}$ & \\
\hline Heredetary & $\begin{array}{l}\text { Erythromelalgia }{ }^{28} \\
\mathrm{Na}_{\mathrm{v}} 1.7 \text {-mutations } \\
\mathrm{Na}_{\mathrm{v}} 1.8 \text {-mutations } \\
\mathrm{Na}_{\mathrm{v}} 1.31 \text {-mutations } \\
\text { Fabry disease }^{32} \\
\text { Ross' syndrome, }^{63} \\
\text { Haemochromatosis }^{64}\end{array}$ & $\begin{array}{c}\text { Familial amyloidosis }{ }^{65} \\
\text { Fabry disease }^{62} \\
\text { Tangier's disease }^{66} \\
\text { Friedreich ataxia }^{67} \\
\text { Cerebrotendinous } \\
\text { xanthomatosis } \\
{ }^{68} \\
\text { Hereditary sensory } \\
\text { autonomic neuropathies }\end{array}$ & \\
\hline Idiopathic & $\begin{array}{l}\text { Idiopathic small fibre neuropathy }{ }^{70} \\
\text { Burning mouth syndrome }\end{array}$ & & $\begin{array}{c}\text { Complex regional } \\
\text { pain syndrome } \\
\text { type }\left.\right|^{72}\end{array}$ \\
\hline $\begin{array}{l}\text { Neuro } \\
\text {-degenerative }\end{array}$ & & & $\begin{array}{c}\text { Spinobulbar } \\
\text { muscular atrophy } \\
\text { (Kennedy's disease) }^{73} \\
\text { Amyotrophic } \\
\text { lateral sclerosis }^{74} \\
\text { Parkinson's disease } \\
\text { Park }\end{array}$ \\
\hline
\end{tabular}




\section{Treatment}

Treatment so far relies on identification of underlying diseases/agents/toxins and appropriate treatment (or cessation of toxin) if possible. This may lead to improvement of complaints. ${ }^{55,76-78}$ Special attention should be given to diabetic patients, as a tight glycemic control may prevent or ameliorate symptoms. ${ }^{17}$ However, rapidly induced glucose regulation invokes a painful neuropathy. ${ }^{35}$

Otherwise, only symptomatic relief of complaints is available. Usually, drugs to reduce neuropathic pain are prescribed such as amitriptylin, pregabalin, carbamazepin. ${ }^{79}$ Unfortunately, only a proportion of patients will respond favorable to these drugs.

\section{Prognosis}

The overall prognosis of a patient, of course, depends on the underlying disease. In idiopathic small fiber neuropathy, spontaneous symptom reduction, stable disease and symptom progression have been described. Also, with varying underlying diseases, progression to mixed fiber neuropathy may occur. ${ }^{20}$ 


\section{REFERENCES}

1. Lacomis D. Small-fiber neuropathy. Muscle Nerve 2002;26:173-188.

2. Lauria G. Small fibre neuropathies. Curr Opin Neurol 2005;18:591-597.

3. Hoeijmakers JG, Faber CG, Lauria G, Merkies IS, Waxman SG. Small-fibre neuropathies-advances in diagnosis, pathophysiology and management. Nat Rev Neurol 2012;8:369-379.

4. Estacion M, Han C, Choi JS, et al. Intra- and interfamily phenotypic diversity in pain syndromes associated with a gain-of-function variant of NaV1.7. Mol Pain;7:92.

5. Gemignani F, Giovanelli M, Vitetta F, et al. Non-length dependent small fiber neuropathy. a prospective case series. J Peripher Nerv Syst 2010;15:57-62.

6. Lauria G, Sghirlanzoni A, Lombardi R, Pareyson D. Epidermal nerve fiber density in sensory ganglionopathies: clinical and neurophysiologic correlations. Muscle Nerve 2001;24:1034-1039.

7. Chong PS, Cros DP. Technology literature review: quantitative sensory testing. Muscle Nerve 2004;29:734747.

8. Yarnitsky D. Quantitative sensory testing. Muscle Nerve 1997;20:198-204.

9. Freeman R, Chase KP, Risk MR. Quantitative sensory testing cannot differentiate simulated sensory loss from sensory neuropathy. Neurology 2003;60:465-470.

10. Arendt-Nielsen L, Chen AC. Lasers and other thermal stimulators for activation of skin nociceptors in humans. Neurophysiol Clin 2003;33:259-268.

11. Ravits JM. AAEM minimonograph \#48: autonomic nervous system testing. Muscle Nerve 1997;20:919-937.

12. Le Quesne PM, Fowler CJ, Parkhouse N. Peripheral neuropathy profile in various groups of diabetics. J Neurol Neurosurg Psychiatry 1990;53:558-563.

13. Stewart JD, Low PA, Fealey RD. Distal small fiber neuropathy: results of tests of sweating and autonomic cardiovascular reflexes. Muscle Nerve 1992;15:661-665.

14. Hoitsma E, Drent M, Verstraete E, et al. Abnormal warm and cold sensation thresholds suggestive of smallfibre neuropathy in sarcoidosis. Clin Neurophysiol 2003;114:2326-2333.

15. Kennedy WR. Opportunities afforded by the study of unmyelinated nerves in skin and other organs. Muscle Nerve 2004;29:756-767.

16. Lauria G, Hsieh ST, Johansson O, et al. European Federation of Neurological Societies/Peripheral Nerve Society Guideline on the use of skin biopsy in the diagnosis of small fiber neuropathy. Report of a joint task force of the European Federation of Neurological Societies and the Peripheral Nerve Society. Eur J Neurol 2010;17:903-912, e944-909.

17. Tesfaye S, Boulton AJ, Dyck PJ, et al. Diabetic neuropathies: update on definitions, diagnostic criteria, estimation of severity, and treatments. Diabetes Care 2010;33:2285-2293.

18. Lauria G, Lombardi R. Small fiber neuropathy: is skin biopsy the holy grail? Curr Diab Rep 2012;12:384-392.

19. Lauria G, Merkies IS, Faber CG. Small fibre neuropathy. Curr Opin Neurol 2012;25:542-549.

20. Devigili G, Tugnoli V, Penza P, et al. The diagnostic criteria for small fibre neuropathy: from symptoms to neuropathology. Brain 2008;131:1912-1925.

21. Bednarik J, Vlckova-Moravcova E, Bursova S, Belobradkova J, Dusek L, Sommer C. Etiology of small-fiber neuropathy. J Peripher Nerv Syst 2009;14:177-183.

22. Danaei G, Finucane MM, Lu Y, et al. National, regional, and global trends in fasting plasma glucose and diabetes prevalence since 1980: systematic analysis of health examination surveys and epidemiological studies with 370 country-years and 2.7 million participants. Lancet 2011;378:31-40.

23. Schmader KE. Epidemiology and impact on quality of life of postherpetic neuralgia and painful diabetic neuropathy. Clin J Pain 2002;18:350-354.

24. Daousi C, MacFarlane IA, Woodward A, Nurmikko TJ, Bundred PE, Benbow SJ. Chronic painful peripheral neuropathy in an urban community: a controlled comparison of people with and without diabetes. Diabet Med 2004;21:976-982.

25. Vlckova-Moravcova E, Bednarik J, Belobradkova J, Sommer C. Small-fibre involvement in diabetic patients with neuropathic foot pain. Diabet Med 2008;25:692-699.

26. Catterall WA. From ionic currents to molecular mechanisms: the structure and function of voltage-gated sodium channels. Neuron 2000;26:13-25. 
27. Drenth JP, Waxman SG. Mutations in sodium-channel gene SCN9A cause a spectrum of human genetic pain disorders. J Clin Invest 2007;117:3603-3609.

28. Davis MD, Weenig RH, Genebriera J, Wendelschafer-Crabb G, Kennedy WR, Sandroni P. Histopathologic findings in primary erythromelalgia are nonspecific: special studies show a decrease in small nerve fiber density. J Am Acad Dermatol 2006;55:519-522.

29. Faber CG, Hoeijmakers JG, Ahn HS, et al. Gain of function Nanu1.7 mutations in idiopathic small fiber neuropathy. Ann Neurol 2012;71:26-39.

30. Faber CG, Lauria G, Merkies IS, et al. Gain-of-function Nav1.8 mutations in painful neuropathy. Proc Natl Acad Sci U S A 2012;109:19444-19449.

31. Huang J, Yang Y, Zhao P, et al. Small-fiber neuropathy Nav1.8 mutation shifts activation to hyperpolarized potentials and increases excitability of dorsal root ganglion neurons. J Neurosci 2013;33:14087-14097.

32. Huang J, Han C, Estacion M, et al. Gain-of-function mutations in sodium channel NaV1.9 in painful neuropathy. Brain 2014.

33. Smith AG, Ramachandran P, Tripp S, Singleton JR. Epidermal nerve innervation in impaired glucose tolerance and diabetes-associated neuropathy. Neurology 2001;57:1701-1704.

34. Sumner CJ, Sheth S, Griffin JW, Cornblath DR, Polydefkis M. The spectrum of neuropathy in diabetes and impaired glucose tolerance. Neurology 2003;60:108-111.

35. Gibbons $\mathrm{CH}$, Freeman R. Treatment-induced diabetic neuropathy: a reversible painful autonomic neuropathy. Ann Neurol 2010;67:534-541.

36. McManis PG, Windebank AJ, Kiziltan M. Neuropathy associated with hyperlipidemia. Neurology 1994;44:2185-2186.

37. Magri F, Buonocore M, Oliviero A, et al. Intraepidermal nerve fiber density reduction as a marker of preclinical asymptomatic small-fiber sensory neuropathy in hypothyroid patients. Eur J Endocrinol 2010;163:279-284.

38. Chao CC, Wu VC, Tan CH, et al. Skin denervation and its clinical significance in late-stage chronic kidney disease. Arch Neurol 2011;68:200-206.

39. Hoitsma E, Marziniak M, Faber CG, et al. Small fibre neuropathy in sarcoidosis. Lancet 2002;359:2085-2086.

40. Chai J, Herrmann DN, Stanton M, Barbano RL, Logigian EL. Painful small-fiber neuropathy in Sjogren syndrome. Neurology 2005;65:925-927.

41. Brannagan TH, 3rd, Hays AP, Chin SS, et al. Small-fiber neuropathy/neuronopathy associated with celiac disease: skin biopsy findings. Arch Neurol 2005;62:1574-1578.

42. Gondim FA, Brannagan TH, 3rd, Sander HW, Chin RL, Latov N. Peripheral neuropathy in patients with inflammatory bowel disease. Brain 2005;128:867-879.

43. Oki Y, Koike H, lijima M, et al. Ataxic vs painful form of paraneoplastic neuropathy. Neurology 2007;69:564-572.

44. Adams D. Hereditary and acquired amyloid neuropathies. J Neurol 2001;248:647-657.

45. Lacomis D, Giuliani MJ, Steen V, Powell HC. Small fiber neuropathy and vasculitis. Arthritis Rheum 1997; $40: 1173-1177$

46. Tseng MT, Hsieh SC, Shun CT, et al. Skin denervation and cutaneous vasculitis in systemic lupus erythematosus. Brain 2006;129:977-985

47. Pan CL, Tseng TJ, Lin YH, Chiang MC, Lin WM, Hsieh ST. Cutaneous innervation in Guillain-Barre syndrome: pathology and clinical correlations. Brain 2003;126:386-397.

48. Lund C, Koskinen M, Suneetha S, et al. Histopathological and clinical findings in leprosy patients with chronic neuropathic pain: a study from Hyderabad, India. Lepr Rev 2007;78:369-380.

49. Bennett JL, Mahalingam R, Wellish MC, Gilden DH. Epstein-Barr virus--associated acute autonomic neuropathy. Ann Neurol 1996:40:453-455.

50. Polydefkis M, Yiannoutsos CT, Cohen BA, et al. Reduced intraepidermal nerve fiber density in HIVassociated sensory neuropathy. Neurology 2002;58:115-119.

51. Tembl Jl, Ferrer JM, Sevilla MT, Lago A, Mayordomo F, Vilchez JJ. Neurologic complications associated with hepatitis C virus infection. Neurology 1999;53:861-864.

52. Younger DS, Orsher S. Lyme Neuroborreliosis: Preliminary Results from an Urban Referral Center Employing Strict CDC Criteria for Case Selection. Neurol Res Int 2010;2010:525206.

53. Cherry CL, Skolasky RL, Lal L, et al. Antiretroviral use and other risks for HIV-associated neuropathies in an international cohort. Neurology 2006;66:867-873. 
54. Giannoccaro MP, Donadio V, Gomis Perez C, Borsini W, Di Stasi V, Liguori R. Somatic and autonomic small fiber neuropathy induced by bortezomib therapy: an immunofluorescence study. Neurol Sci 2011;32:361-363.

55. Tan CH, Chen YF, Chen CC, Chao CC, Liou HH, Hsieh ST. Painful neuropathy due to skin denervation after metronidazole-induced neurotoxicity. J Neurol Neurosurg Psychiatry 2011;82:462-465.

56. Burakgazi AZ, Polydefkis M, Hoke A. Skin biopsy-proven flecainide-induced neuropathy. Muscle Nerve 2012:45:144-146.

57. Tan IL, Polydefkis MJ, Ebenezer GJ, Hauer P, McArthur JC. Peripheral nerve toxic effects of nitrofurantoin. Arch Neurol 2012;69:265-268.

58. Zambelis T, Karandreas N, Tzavellas E, Kokotis P, Liappas J. Large and small fiber neuropathy in chronic alcohol-dependent subjects. J Peripher Nerv Syst 2005;10:375-381.

59. Koike $H$, lijima $M$, Sugiura $M$, et al. Alcoholic neuropathy is clinicopathologically distinct from thiaminedeficiency neuropathy. Ann Neurol 2003;54:19-29.

60. Bernstein AL. Vitamin B6 in clinical neurology. Ann N Y Acad Sci 1990;585:250-260.

61. Han C, Hoeijmakers JG, Ahn HS, et al. Nav1.7-related small fiber neuropathy: impaired slow-inactivation and DRG neuron hyperexcitability. Neurology 2012;78:1635-1643.

62. Scott $L$, Griffin JW, Luciano C, et al. Quantitative analysis of epidermal innervation in Fabry disease. Neurology 1999;52:1249-1254.

63. Bergmann I, Dauphin M, Naumann M, et al. Selective degeneration of sudomotor fibers in Ross syndrome and successful treatment of compensatory hyperhidrosis with botulinum toxin. Muscle Nerve 1998;21:1790-1793.

64. Wouthuis SF, van Deursen CT, te Lintelo MP, Rozeman CA, Beekman R. Neuromuscular manifestations in hereditary haemochromatosis. J Neurol 2010;257:1465-1472.

65. Plante-Bordeneuve V, Said G. Familial amyloid polyneuropathy. Lancet neurology 2011;10:1086-1097.

66. Gibbels E, Schaefer HE, Runne U, Schroder JM, Haupt WF, Assmann G. Severe polyneuropathy in Tangier disease mimicking syringomyelia or leprosy. Clinical, biochemical, electrophysiological, and morphological evaluation, including electron microscopy of nerve, muscle, and skin biopsies. J Neurol 1985;232:283-294.

67. Nolano M, Provitera V, Crisci C, et al. Small fibers involvement in Friedreich's ataxia. Annals of neurology 2001;50:17-25.

68. Chen SF, Tsai NW, Chang CC, et al. Neuromuscular abnormality and autonomic dysfunction in patients with cerebrotendinous xanthomatosis. BMC Neurol 2011;11:63.

69. Houlden H, Blake J, Reilly MM. Hereditary sensory neuropathies. Current opinion in neurology 2004;17:569577.

70. Gorson KC, Ropper AH. Idiopathic distal small fiber neuropathy. Acta Neurol Scand 1995;92:376-382.

71. Lauria G, Majorana A, Borgna M, et al. Trigeminal small-fiber sensory neuropathy causes burning mouth syndrome. Pain 2005;115:332-337.

72. Oaklander AL, Rissmiller JG, Gelman LB, Zheng L, Chang Y, Gott R. Evidence of focal small-fiber axonal degeneration in complex regional pain syndrome-I (reflex sympathetic dystrophy). Pain 2006;120:235243.

73. Manganelli F, lodice $V$, Provitera $V$, et al. Small-fiber involvement in spinobulbar muscular atrophy (Kennedy's disease). Muscle Nerve 2007;36:816-820

74. Weis J, Katona I, Muller-Newen G, et al. Small-fiber neuropathy in patients with ALS. Neurology 2011;76:2024-2029.

75. Rossi A, Giovenali P, Benvenuti M, Di lorio W, Calabresi P. Skin biopsy: a new diagnostic tool for autonomic dysfunctions in Parkinson's disease? Lancet neurology 2007;6:848-849; author reply 849.

76. Chao CC, Sun HY, Chang YC, Hsieh ST. Painful neuropathy with skin denervation after prolonged use of linezolid. BMJ Case Rep 2009;2009.

77. Morozumi S, Kawagashira Y, lijima M, et al. Intravenous immunoglobulin treatment for painful sensory neuropathy associated with Sjogren's syndrome. J Neurol Sci 2009;279:57-61.

78. Penza P, Lombardi R, Camozzi F, Ciano C, Lauria G. Painful neuropathy in subclinical hypothyroidism: clinical and neuropathological recovery after hormone replacement therapy. Neurol Sci 2009;30:149-151.

79. Attal N, Cruccu G, Baron R, et al. EFNS guidelines on the pharmacological treatment of neuropathic pain: 2010 revision. Eur J Neurol 2010;17:1113-e1188. 


\section{Chapter 1.2}

\section{OUTLINE OF THE THESIS}

In this thesis, the diagnostic approach of small fiber neuropathy will be presented, followed by a discussion of some clinical and clinimetric aspects.

In chapter two, first, Dutch normative values for intraepidermal nerve fiber density at the distal leg are presented. The normative values are validated for clinical use by comparing the healthy subjects with sarcoidosis patients with and without small fiber neuropathy. Second, it is described how reference data for intraepidermal nerve fiber density at the distal leg from nine collaborative laboratories around the world are pooled and examined, validating our institutional findings, and creating worldwide clinically applicable IENFD normative values.

A systematic review of temperature threshold testing is presented in chapter three, discussing the great variability that exists in assessing TTT with regard to basic equipment, testing methodology, and site of examination. It provides a series of recommendations to improve its future use in patients with peripheral neuropathies. The evaluation and optimization of the TTT protocol at the MUMC is thereafter discussed, aiming for a more time efficient and patient friendly (with less burden) protocol.

Normative values for contact heat evoked potentials, a possible complimentary diagnostic test, are presented in chapter four.

The incidence and prevalence of small fiber neuropathy are important to recognize the impact on population level and the results of a survey are presented in chapter five.

In chapter six pain and autonomic dysfunction, key features in small fiber neuropathy are evaluated in patients with SFN.

Last but not least, in chapter seven impact of small fiber neuropathy on quality of life is discussed.

To provide a systematic overview, chapters eight and nine contain a summary and general discussion, concluding with future perspectives.

Finally, chapter ten deals with valorization, the practical implementation of this study. 

PART II

DIAGNOSIS OF SMALL FIBER NEUROPATHY 

Chapter 2.1

\section{INTRAEPIDERMAL NERVE}

FIBER DENSITY AND

ITS APPLICATION IN SARCOIDOSIS

M. Bakkers', I.S.J. Merkies ${ }^{1,2,3}$, G. Lauria ${ }^{4}$, G. Devigili ${ }^{5}$, P. Penza ${ }^{4}$, R. Lombardi ${ }^{4}$, M.C.E. Hermans', S.I. van Nes ${ }^{3}$, M. De Baets ${ }^{6}$, C.G. Faber'

'Department of Neurology, Maastricht University Medical Center, the Netherlands

2Department of Neurology, Spaarne Hospital, the Netherlands ${ }^{3}$ Department of Neurology, Erasmus Medical Center Rotterdam, the Netherlands ${ }^{4}$ Neuromuscular Diseases Unit, IRCCS Foundation, "Carlo Besta” National Neurological Institute, Milan, Italy ${ }^{5}$ Neurologic Clinic, University of Ferrara, Ferrara, Italy ${ }^{6}$ School for Mental Health and Neuroscience, the Netherlands. 


\section{ABSTRACT}

Background: Intraepidermal nerve fiber density (IENFD) is considered a good diagnostic tool for small fiber neuropathy (SFN).

Objectives: To assess stratified normative values for IENFD, and determine its reliability and validity in sarcoidosis.

Methods: IENFD was assessed in 188 healthy volunteers and 72 sarcoidosis patients ( $n=58$ with SFN symptoms, $n=14$ without SFN symptoms). Healthy controls were stratified (for age and gender), resulting in six age groups (20-29, 30-39,...up to $\geq 70$ years) containing at least 15 men and 15 women. A skin biopsy was taken in each participant $10 \mathrm{~cm}$ above the lateral malleolus and analyzed in accordance with the international guidelines using bright-field microscopy. Interobserver/intraobserver reliability of IENFD was examined. In the patients, a symptoms inventory questionnaire (SIQ; assessing SFN symptoms) and the Vickrey Peripheral Neuropathy Quality-of-Life Instrument-97 (PNQoL-97) were assessed to examine the discriminative ability of normative IENFD values.

Results: There was a significant age-dependent decrease of IENFD values in healthy controls, with lower densities in men compared with women. Good interobserver/ intraobserver reliability scores were obtained ( $k$ values $\geq 0.90$ ). A total of 21 patients with sarcoidosis had a reduced IENFD score $\left(<5^{\text {th }}\right.$ percentile; 19 [32.8\%] in patients with SFN symptoms, 2 [14.3\%] in patients without SFN symptoms). The validity of the normative IENFD values was demonstrated by distinguishing between the SIQ scores and various PNQoL-97 values for the different patient groups.

Conclusion: This study provides clinically applicable distal intraepidermal nerve fiber density normative values, showing age-and sex-related differences. 


\section{INTRODUCTION}

Investigating sensory symptoms can be a diagnostic challenge. When a neuropathy is considered, neurologic examination and nerve conduction studies generally do not reveal abnormalities if predominantly small fibers are affected. ' There is no gold standard for diagnosing small fiber neuropathy (SFN), though a sensory neuropathy may present with small fiber involvement or indicate a disease causing pure small fiber dysfunction. ${ }^{2}$ Typically, patients with SFN report burning pain in the extremities, combined with autonomic dysfunction, such as dry eyes, changes in sweating, and gastrointestinal and urogenital dysfunction. ${ }^{3}$

Quantitative sensory testing such as temperature detection thresholds, sudomotor axon reflex, and cardiovascular autonomic testing are available techniques to determine small nerve fiber function. However, some tests have disadvantages, such as subjectivity, high burden for patients, or low sensitivity and specificity. ${ }^{4}$ In SFN, loss of intraepidermal nerves is considered an objective diagnostic feature. ${ }^{5}$

There are several potentially treatable illnesses that may cause SFN. Diabetes mellitus is the most frequent underlying disease, but several other metabolic, inflammatory, and hereditary disorders cause SFN. ${ }^{2}$ Recently, SFN was demonstrated to occur in sarcoidosis. ${ }^{6}$ Only a small sample of patients with sarcoidosis has been examined so far.

Determination of intraepidermal nerve fiber density (IENFD) is considered a reliable technique to assess loss and regeneration of small nerve fibers. Several techniques have been adopted; the most commonly used are bright field immunohistochemistry and indirect immunofluorescence on punch biopsies. ${ }^{7-9}$ Reported interobserver/ intraobserver variability is good..$^{10}$

The aims of the current study are to present normative IENFD values, stratified for age and sex, for a collaborative group (Maastricht, The Netherlands; Milan and Ferrara, Italy), and to examine the interobserver/intraobserver reliability and discriminatory validity of IENFD in a cohort of patients with sarcoidosis.

\section{METHODS}

\section{Participants}

Healthy controls

Healthy controls were recruited from hospital personnel, from relatives and friends of patients, at sports accommodations, and at informal meetings for the elderly, in The Netherlands and Italy. Healthy individuals were stratified for age and sex, forming 6 age groups (20-29, 30-39, 40-49,...up to 70 years and older) consisting of at least 15 men and 15 women each. Eligibility criteria were lucid consciousness, no neurologic symptoms, no history of alcohol misuse (defined as $>4$ IU/day), no use of drugs or history of diseases that may cause sensory deficit (e.g., chemotherapeutic agents in 
the past, malignancy, diabetes mellitus, thyroid dysfunction, renal failure, peripheral vascular disease, skin disorders), and normal findings at neurologic examination.

Patients

From October 2006 to June 2008, patients referred to the Maastricht Sarcoidosis Management Center, a referral center for sarcoidosis in The Netherlands, were screened for eligibility. Inclusion criteria for the study were age older than 18 years, no alcohol abuse, no use of immunosuppressant or neuropathy-causing drugs, no concomitant disease possibly causing SFN (normal laboratory findings for glucose, thyroid function, blood count, and renal and liver function tests), and a neurologic examination without signs of large fiber neuropathy or CNS involvement. Furthermore, nerve conduction findings (assessing median, peroneal, and tibial motor nerves, and median and sural sensory nerves under predefined conditions) had to be normal. All patients were diagnosed as having sarcoidosis in accordance with postulated diagnostic guidelines. ${ }^{11}$ We only recruited therapy-naive patients or patients who had not received immuno -suppressive therapy in the 6 months before inclusion, because the effect of these medications on small nerve fibers is unknown.

\section{Assessments}

Skin biopsy

Published European guidelines on the use of skin biopsy in the diagnosis of SFN were used. ' A 3-mm punch biopsy was performed under local anesthesia (1\% lidocaine) $10 \mathrm{~cm}$ above the right lateral malleolus in all subjects. All biopsies were fixed in $2 \%$ paraformaldehyde-lysinesodiummetaperiodate and stored at $-80^{\circ} \mathrm{C}$ in a cryoprotective solution (20\% glycerol) before further processing. After cutting (Microm, HM560 Cryostar, Walldorf, Germany), the $50 \mu$ thick sections were immunostained using a free-floating method: after bleaching, the sections were blocked with goat serum, incubated with the panaxonal marker PGP 9.5 (Ultraclone, Wellow, Isle of Wight, UK), and secondary antibody (biotinylated anti-rabbit immunoglobulin G, Biozol, Eching, Germany). The reaction was then visualized after endogenous peroxidase blocking, with the Vectastain ABC kit and Vector SG substrate kit (Vector Laboratories, Burlingame, CA). Epidermal length was measured and sections were counted with bright field microscopy on a stereology workstation (Olympus BX50 with PlanApo objective 40x[oil; NA=1.0], Tokyo, Japan, and stereology software Stereo-Investigator version 7.00.3, Micro-BrightField, Williston, VT), applying the established counting rules. $^{12}$ In brief, the rules state that to be counted, intraepidermal nerves have to cross or originate at the dermal-epidermal junction, and secondary branches and fragments are not counted. After fixing the specimens, at least 3 sections were analyzed under the light microscope. IENFD is reported as the mean IENF of these 3 sections per millimeter.

SFN symptoms inventory questionnaire

A simple SFN symptoms inventory questionnaire (SIQ) was constructed, based on 
international guidelines. ${ }^{13,14}$ The SIQ includes 12 inquiries addressing the following aspects: presence of palpitations, flushes, constipation or diarrhea, urination problems (incontinence or hesitation), changes in sweating pattern, restless legs, orthostatic dizziness, dry eyes or mouth, oversensitivity and sheet intolerance of the legs. Items focused on the presence or absence of these symptoms ( 0 "no symptoms" to 12 "maximum number of symptoms").

Vickrey Peripheral Neuropathy Quality-of-Life Instrument-97

The Vickrey Peripheral Neuropathy Qualityof-Life Instrument-97 (PNQoL-97) has the Short-Form 36ltem Health Survey as a generic core, supplemented with specifically neuropathy related questions, resulting in a 97-item scale, with 91 items distributed among 16 multiscale domains and 6 separately scored questions. ${ }^{15,16}$ The domains are physical functioning (11 items), role limitations due to physical health problems (6 items), pain (7), energy/fatigue (5), upper extremities (6), balance (8), self-esteem (6), emotional well-being (7), stigma (3), cognitive function (3), role limitations due to emotional problems (3), general health perceptions (7), sleep (5), social functioning (9), sexual function (2), and health distress (3). ${ }^{16}$ Each domain has a scoring range from 0 to 100. A high score indicates better health. For the purpose of the current study, the original PNQoL-97 was translated-back-translated by 2 independent translators into Dutch in accordance with international guidelines. ${ }^{14}$ Ambiguous items were discussed until uniformity was obtained on all items. The Vickrey PNQoL-97 physical and mental component summary score (V-PCS, V-MCS) values were calculated. ${ }^{16-18}$

\section{Standard protocol approvals, registration, and patient consent}

The study was approved by the medical ethical committees of all participating centers and the CCMO (Central Committee for Human Related Research, identifier number p06.0066L/MEC 05-224). Written informed consent was obtained from all participants before inclusion.

\section{Study design}

General aspects

Examination occurred in a comfortable, temperature-controlled room. All participants underwent a standardized interview, neurologic examination, and skin biopsy by a skilled investigator (M.B.). All selected patients were recruited at the department of neurology of the Maastricht University Medical Center (MUMC), The Netherlands. A patient was classified as having SFN symptoms when he or she reported at least 1 of the following symptoms, not otherwise explained: burning pain in extremities, dry mouth or eyes, changes in sweating, flushes, gastrointestinal dysfunction (constipation, diarrhea), cardiac symptoms (palpitation, dizziness on standing up), and urogenital dysfunction (sexual dysfunction, incontinence). Those without any of these symptoms were classified as having no SFN symptoms (patient control group). All patients completed the SIQ and PNQoL-97. 
Reliability studies

All biopsies were coded, thus blinding the examiners. The IENFD of all participants at the MUMC was counted by 1 researcher (M.B.). Fifty randomly selected slides (25 patients plus 25 healthy controls) were also analyzed by a second examiner (C.G.F.) (local IENFD interobserver reliability). These 50 slides were recounted by the examiners (interval at least 2 weeks) for the IENFD intraobserver reliability scores of both examiners, without having access to the earlier findings. To determine interobserver reliability between laboratories, examiners at Milan (R.L.), Ferrara (G.D.), and Maastricht (M.B.) each counted 30 arbitrarily selected slides (healthy controls; 10 slides per center, circulating between institutes).

The SIQ was completed twice (interval: 2-4 weeks) to examine its test-retest reliability. Validity studies

The validity of the IENFD normative values was examined through discriminatory studies with the SIQ and PNQOL-97 domains and component sum scores in the patient group. In particular, we examined whether the normative data would help to discriminate between the obtained scores in these outcome measures that are based on patients' own perception (sarcoidosis patients without SFN symptoms vs those patients with SFN symptoms but normal IENFD scores vs those with SFN symptoms and abnormal IENFD).

\section{Statistical analysis}

Data handling and normative values

Data collection, entry, and management were performed using the Teleform automated processing system, striving to reduce the number of manual data entry errors. ${ }^{19}$ All analyses were performed using Stata 10.0 for Windows XP (StataCorp LP, College Station, TX). A value of $p<0.05$ was considered significant.

Normative IENFD reference values were calculated in healthy controls (5th percentile values, corresponding to a chosen specificity of 95\%), depending primarily on age and sex. Quantile regression analyses were performed to obtain the normative data. A patient's IENFD was considered to be abnormal if the corresponding value was below the normative data 5th percentile lower limit, because this cutoff value has previously been used. ${ }^{20}$ Weight and height were analyzed for possible correlating confounding impact on IENFD normative values.

Reliability studies

Interobserver and intraobserver reliability scores for the IENFD and SIQ test-retest reliability were determined using weighted $\mathrm{k}$ statistic measures. ${ }^{21}$ The weights of the were defined as $1-[(i-j) /(k-1)]^{2}(i=$ rows and $j=$ columns of the ratings by two observers or by the patients twice in time, $k=$ maximum number of possible ratings).

Validity studies

The discriminative capacity of the obtained IENFD normative values was investigated in 3 subgroups of patients (subgroup A: sarcoidosis patients without SFN symp- 
toms; subgroup B: sarcoidosis patients with SFN symptoms but normal IENFD values; subgroup C: sarcoidosis patients with SFN symptoms and impaired IENFD scores $\left[<5^{\text {th }}\right.$ percentile lower limit]) by comparing the obtained scores for SIQ plus PNQOL-97 values in the patient subgroups (analysis of variance with corrections according to Bonferroni multiple comparison tests for continuous variables; $X^{2}$-test for ordinal variables).

Chapter

2.1

\section{RESULTS}

\section{General aspects}

Recruitment was performed between January 2006 and June 2008. Skin biopsies were collected in 188 healthy volunteers (Maastricht $n=105$, Milan $n=44$, Ferrara $n=39$ ), stratifying for age decade and sex (15 men and 15 women per age decade, range 20-82 years and older, forming 6 age decades). Most volunteers were white (97\%).

Between October 2006 and June 2008, 211 patients with sarcoidosis were referred to the MUMC (see Figure 1). Reasons for exclusion were immunosuppressive treatment (65), comorbidity that could cause neuropathy (30), CNS involvement (11) and large nerve fiber involvement found at neurologic or nerve conduction studies (21), and inability or unwillingness to attend examination (9). Eventually, 72 patients met the inclusion criteria and declared willingness to participate. Of these 72 patients, 58 were categorized as having symptoms not otherwise explained and possibly caused by SFN, and 14 were categorized as not having any SFN-related symptoms. All patients mentioned fatigue as one of their main problems. The participants' characteristics are presented in Table 1. No complications were encountered in either healthy volunteers or patients due to the skin biopsy.

\section{Normative values}

Normative IENFD reference values were calculated in 188 healthy controls. There was a significant age-dependent decrease of IENFD values, with lower scores for men compared with women (Figure 2).

Height and weight did not have any significant impact on the IENFD scores. The obtained normative scores were subsequently translated per age span and sex for clinical use, using the 5 th percentile as a cutoff value (Table 2). 
Figure 1. Inclusion flowchart.

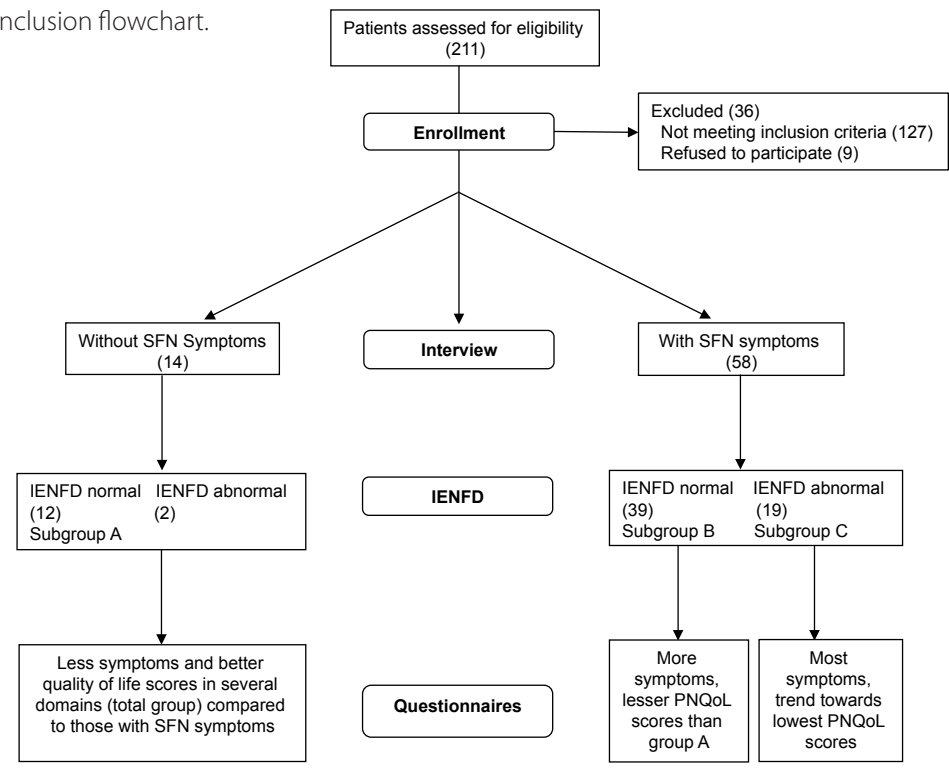
We cannot provide a flowchart for the inclusion of the healthy controls, as we addressed people also
at meetings, specifically mentioning exclusion criteria, and did not register the number of people
attending. So those that we examined were already a subset of those that were approached.

Table 1. Basic characteristics of healthy controls and sarcoidosis patients, with or without small fiber neuropathy complaint.

Healthy controls Sarcoidosis patients Sarcoidosis patients with SFN complaints without SFN complaints

\begin{tabular}{|c|c|c|c|}
\hline Number & 188 & 58 & 14 \\
\hline \multicolumn{4}{|l|}{ Gender (\%) } \\
\hline Female & $97(51,6 \%)$ & $25(43,1 \%)$ & $7(50 \%)$ \\
\hline Male & $91(48,4 \%)$ & $33(56,9 \%)$ & $7(50 \%)$ \\
\hline Age, mean years (SD), range & 47.0 (15.8), 20-82 & $45.4(9.2), 29-70$ & $46.5(9.5), 27-65$ \\
\hline $\begin{array}{l}\text { Duration of complaints in years (SD), } \\
\text { range }\end{array}$ & - & $7.9(6.0), 1-32$ & $9.4(7.3), 2-28$ \\
\hline Main initial reported symptom ${ }^{1}$ & - & $\begin{array}{l}\text { Burning feet 1.7\% } \\
\text { Pain } 22.6 \% \\
\text { Pulmonary } 32.8 \% \\
\text { Fatigue } 6.9 \%\end{array}$ & $\begin{array}{c}\text { Pain }^{2} \text { 28.6\% } \\
\text { Pulmonary } 28.6 \% \\
\text { Fatigue } 7.1 \%\end{array}$ \\
\hline Main current reported symptoms ${ }^{1}$ & - & $\begin{array}{c}\text { Burning feet } 29.3 \% \\
\text { Pain }^{2} 53.4 \% \\
\text { Pulmonary } 6.9 \% \\
\text { Fatigue } 96.6 \%\end{array}$ & $\begin{array}{c}- \\
\text { Pain }^{2} 28.5 \% \\
\text { Pulmonary } 42.9 \% \\
\text { Fatigue } 85.7 \%\end{array}$ \\
\hline
\end{tabular}

${ }^{1}$ Does not add up to $100 \%$ due to not separately mentioned symptoms (skin abnormalities, different autonomic symptoms, uveitis, fever and weight loss).

${ }^{2}$ Various pain modalities were addressed: thoracic pain, joint pain, other pain in extremities such as muscle pain.

${ }^{3}$ Dispnea and cough were mentioned as the main pulmonary complaints. 
Figure 2. IENFD of 188 healthy individuals.
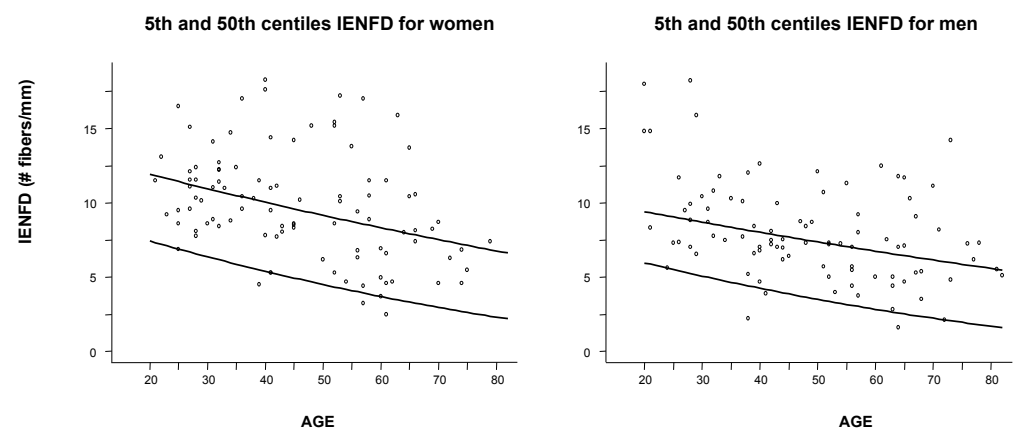

Chapter

2.1

Scatter plot showing normative intra epidermal nerve fiber density (IENFD) scores in healthy individuals stratified for age and gender ( $5^{\text {th }}$ and $50^{\text {th }}$ centiles shown).

Table 2. Intra-epidermal nerve fiber density (IENFD) normative values for clinical use.

\begin{tabular}{lcccc}
\hline & \multicolumn{2}{c}{ Females (97) } & \multicolumn{2}{c}{ Males (91) } \\
\hline Age (years) & $\begin{array}{l}\text { 0.05 quantile IENFD } \\
\text { values per age span }\end{array}$ & $\begin{array}{c}\text { median } \\
\text { IENFD values } \\
\text { per age span }\end{array}$ & $\begin{array}{c}\mathbf{0 . 0 5} \text { quantile IENFD } \\
\text { values per age span }\end{array}$ & $\begin{array}{c}\text { median } \\
\text { IENFD values } \\
\text { per age span }\end{array}$ \\
\hline $20-29$ & 6.7 & 11.2 & 5.4 & 9.0 \\
$30-39$ & 6.1 & 10.7 & 4.7 & 8.4 \\
$40-49$ & 5.2 & 9.9 & 4.0 & 7.8 \\
$50-59$ & 4.1 & 8.7 & 3.2 & 7.1 \\
$60-69$ & 3.3 & 7.9 & 2.4 & 6.3 \\
$\geq 70$ & 2.7 & 7.2 & 2.0 & 5.9 \\
\hline
\end{tabular}

\section{Reliability and discriminative validity studies}

Good intraobserver reliability values were obtained for both researchers in Maastricht (MB.: weighted $\mathrm{k}=0.95$; C.G.F.: 0.90), with good interobserver scores as well (0.94). There was also a good interobserver reliability between the examiners at the 3 institutes: Maastricht-Milan: weighted $\mathrm{k}=0.78$; Maastricht-Ferrara: 0.83; Milan-Ferrara: 0.91. In the subgroup of patients with sarcoidosis and possible SFN symptoms $(n=58)$, 19 patients (32.8\%) had impaired IENFD scores. In contrast, all patients without SFN symptoms except 2 (14.3\%) had normal IENFD values ( $X^{2}$-test: $\left.p=0.17\right)$. For further discriminative validity studies, we divided the patients with SFN symptoms into those with IENFD being normal and those with IENFD $<5$ th percentile lower limit, ultimately having 3 subgroups for comparison (subgroup A: sarcoidosis without SFN symptoms, $n=14$; subgroup B: sarcoidosis with SFN symptoms, normal IENFD findings, $n=39$; subgroup C: sarcoidosis with SFN symptoms and abnormal IENFD values, $\mathrm{n}=19$ ). Figure 3 illustrates that more SFN-related symptoms as reported by SIQ are present in patients with abnormal IENFD, with a gradual transition between the 3 subgroups ( $X^{2}-$ test between subgroups: $p<0.001)$. Hypersensitivity is reported by $14 \%$ in subgroup $A$, 
by $69 \%$ in subgroup $B$, and by $79 \%$ in subgroup C. Similar differences for symptoms of dry mouth $(50 \%, 72 \%, 79 \%)$ and orthostatic intolerance $(36 \%, 67 \%, 84 \%)$ were found. Presence of SFN symptoms in subgroup A was comparable to that in healthy controls (data not shown). The SIQ demonstrated good test-retest reliability (weighted $\mathrm{k}=0.88$ ).

Figure 3. Discriminative validity of IENFD values related to the symptoms inventory questionnaire (SIQ) score in sarcoidosis.

\section{Reported SFN complaints by patients with sarcoidosis using a symptoms inventory questionnaire}

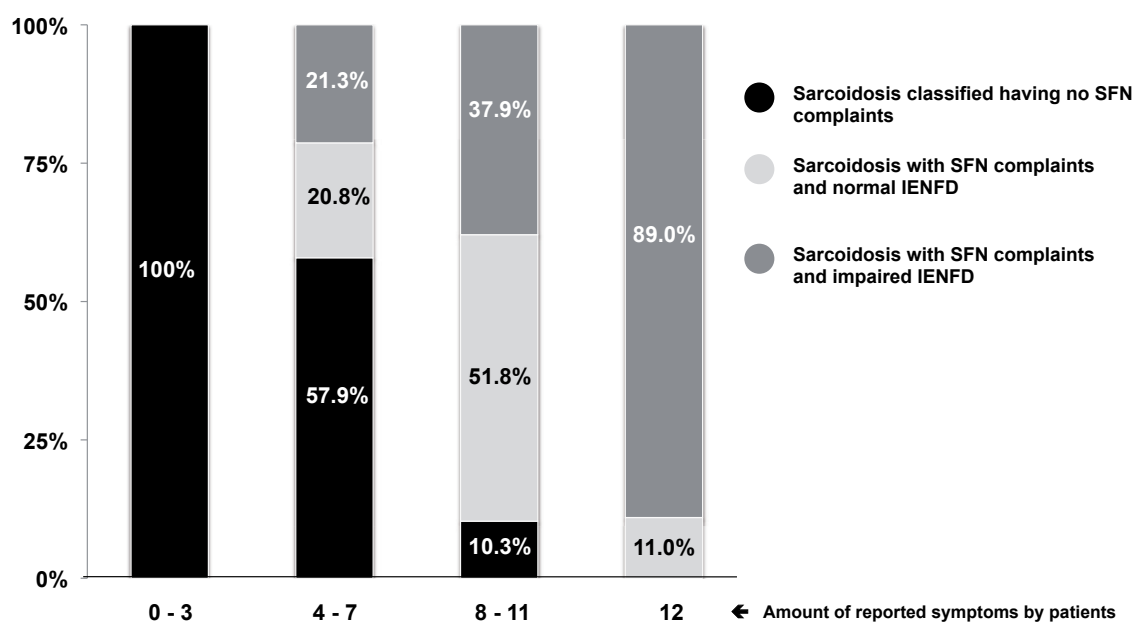

Bar chart showing patients with few (0-3), some (4-7), al lot (8-11) and all 12 symptoms, divided per subgroup: Subgroup A, black $(n=14)$ : patients classified having no SFN complaints; Subgroup B, white $(n=39)$ : patients classified having SFN symptoms, but normal IENFD at examination; Subgroup C, grey ( $\mathrm{n}=19$ ): patients classified having SFN symptoms and abnormal IENFD.

A significant discriminative validity was obtained when comparing the results of the PNQoL-97 domains between the 3 subgroups for pain, balance, energy/fatigue, upper extremity function, cognitive functioning, and social functioning. Also, the physical component score of this scale (V-PCS) showed a discriminative trend between the 3 subgroups of patients (Table 3). In general, these PNQoL-97 domains and V-PCS did not significantly differentiate between subgroups B and C, patients with SFN symptoms, but showed a trend of more reduced quality-of-life features in those with abnormal IENFD (Table 3). The remaining domains and V-MCS did not show any difference between the subgroups of patients. 
Table 3. Comparison of PNQoL domain scores between subgroups of sarcoidosis patients.

\begin{tabular}{|c|c|c|c|c|}
\hline Domain & subgroup comparison ${ }^{1}$ & $A$ versus $B^{2}$ & $A$ versus $C^{2}$ & $B$ versus $C^{2}$ \\
\hline Pain & $p<0.0001$ & $p<0.0001$ & $p<0.0001$ & $p=0.057$ \\
\hline Balance & $p<0.0001$ & $p=0.003$ & $p<0.0001$ & $\mathrm{p}=\mathrm{NS}^{3}$ \\
\hline Energy/fatigue & $p=0.01$ & $\mathrm{p}=\mathrm{NS}$ & $p=0.02$ & $p=N S$ \\
\hline $\begin{array}{l}\text { Upper } \\
\text { extremities } \\
\text { function }\end{array}$ & $p=0.004$ & $p=0.01$ & $p=0.005$ & $\mathrm{p}=\mathrm{NS}$ \\
\hline $\begin{array}{l}\text { Cognitive } \\
\text { functioning }\end{array}$ & $p=0.04$ & $p=0.01$ & $\mathrm{p}=\mathrm{NS}$ & $\mathrm{p}=\mathrm{NS}$ \\
\hline $\begin{array}{l}\text { Social } \\
\text { functioning }\end{array}$ & $p=0.04$ & $p=0.03$ & $p=N S$ & $p=N S$ \\
\hline $\begin{array}{l}\text { Physical } \\
\text { component } \\
\text { score }\end{array}$ & $p=0.0004$ & $p=0.002$ & $p<0.0001$ & $\mathrm{p}=\mathrm{NS}$ \\
\hline
\end{tabular}

'Overall result from ANOVA with corrections according to Bonferroni multiple-comparison tests.

${ }^{2}$ Subgroup A: patients without SFN related symptoms; subgroup B: patients with SFN related symptoms and normal IENFD; subgroup C: patients with SFN related symptoms and reduced IENFD.

${ }^{3} \mathrm{NS}=$ Not significant.

\section{DISCUSSION}

In the current study, we described the determination of the largest series of normative distal leg IENFD values, stratified for age and sex. We demonstrated good interobserver/ intraobserver reliability and acceptable IENFD validity scores through association with questionnaires. This series of skin biopsies is available for other researchers interested in joining a quality control program and in enlarging their normative reference values. Normative data for IENFD have been reported previously for the same location. ${ }^{20,22-24}$ Our results differ (lower values) from previous reports on IENFD normative values, which is most probably explained by differences in sample size of healthy controls and in count ing rules. We found lower IENFD values in men compared with women, and an agerelated decrease in IENFD, which is in conformity with these earlier reports.

Several neurologic tests have shown decreased function in healthy elderly, such as diminished grip strength, temperature and vibration sensation, and nerve conduction studies. ${ }^{25-28}$ Also, women have been reported to be more sensitive in quantitative sensory testing. ${ }^{29,30}$ On the structural level, age-related decline is noted too, for example, in Meissner and Pacinian corpuscles. ${ }^{31,32}$ The reasons for age-related decline and sex differences may be related to differences in hormonal status. ${ }^{33}$ Progesterone and its metabolites, for example, stimulate axonal growth and myelinization. Though it is also synthesized in the nervous system, in female rats, it is more abundant because of the gonadal production, and in experimental studies, nerve sprouting, regeneration, and remyelinization are more rapid and more complete in female animals.34,35

In our series, we were able to identify one-third of sarcoidosis patients with SFN using 
only sex-and age-related cutoff IENFD normative values. Our IENFD sensitivity turned out to be lower compared with reported literature findings, which is most probably related to differences in population of patients examined and differences in IENFD cutoff values. ${ }^{36-38}$ We only included patients with pure small fiber-related symptoms and excluded patients with large fiber neuropathy. Others have examined patients with sensory neuropathy symptoms (thus having thick nerve fiber involvement) and have demonstrated higher sensitivity of reduced IENFD in these patients compared with those with only small nerve fiber involvement. ${ }^{37}, 38$ Progression from pure SFN to large fiber involvement in patients over time with further reduction of IENFD in these patients has also been reported, hence underlining the notion of a continuum and progressive disease pattern. ${ }^{36}$

The current article addresses discriminatory validity between subgroups of patients with sarcoidosis, translating pathology (small fiber findings in skin biopsy) to impairments (SFN symptoms) and partly to quality-of-life expectations, by demonstrating differences between sarcoidosis patients without SFN symptoms vs those with SFN symptoms but normal IENFD vs those with SFN symptoms and impaired IENFD. Similar findings have been reported in patients with various forms of inflammatory neuropathies. ${ }^{39}$ Although not significant, patients with SFN symptoms and impaired IENFD had more physically oriented symptoms, such as more pain, less energy, and more balance problems, and more social dysfunction compared with the other subgroups. Emotional items scores did not differ between subgroups, thus excluding emotional problems as an explanation for patients' dysfunction. Hence, low IENFD contributes to the patients' daily symptoms and physical burden and helps clinicians in understanding its impact in patients with sarcoidosis.

There are some methodologic issues that should be addressed. First, a gold standard for the diagnosis SFN was proposed: the presence of at least 2 abnormal results at clinical, QST, and skin biopsy examinations. ${ }^{36} \mathrm{However}$, the validation of this proposition is beyond the scope of the current study. Second, some caution is required when interpreting the results, because the sample sizes of the various subgroups were relatively small. Third, efforts were taken to subdivide the 72 selected patients into 3 subgroups based on a systematic interview focusing on the presence of SFN symptoms. Despite these efforts, patients of the subgroup without any SFN symptoms (subgroup A) reported through the SIQ questionnaire some presence of symptoms, although relatively low compared with the other 2 subgroups of patients (Figure 3). However, the presence of symptoms in subgroup A was similar to that in healthy controls. Finally, we have not systematically examined whether skin type or occupation might have any influence on IENFD.

The current study presents clinically applicable distal leg IENFD values, supporting previously reported age-and sex-related differences. Validity through association with an SFN-specific and generic quality-of-life questionnaire was acceptable. Hence, IENFD is suggested as an alternative endpoint in future clinical trials in neuropathies, as has been addressed previously. ${ }^{40}$ Further study in patients with SFN and validation of a possible gold standard are recommended. 


\section{REFERENCES}

1. Mendell JR, Sahenk Z. Clinical practice. Painful sensory neuropathy. N Engl J Med 2003;348:1243-1255.

2. Lauria G. Small fibre neuropathies. Curr Opin Neurol 2005;18:591-597.

3. Lacomis D. Small-fiber neuropathy. Muscle Nerve 2002;26:173-188.

4. Hoitsma E, Reulen JP, de Baets M, Drent M, Spaans F, Faber CG. Small fiber neuropathy: a common and important clinical disorder. J Neurol Sci 2004;227:119-130.

5. Lauria G, Lombardi R. Skin biopsy: a new tool for diagnosing peripheral neuropathy. Bmj 2007;334:11591162.

6. Hoitsma E, Marziniak M, Faber CG, et al. Small fibre neuropathy in sarcoidosis. Lancet 2002;359:20852086.

7. Lauria G, Cornblath DR, Johansson O, et al. EFNS guidelines on the use of skin biopsy in the diagnosis of peripheral neuropathy. Eur J Neurol 2005;12:747-758.

8. Sommer C, Lauria G. Skin biopsy in the management of peripheral neuropathy. Lancet neurology 2007;6:632-642.

9. Ebenezer GJ, Hauer P, Gibbons C, McArthur JC, Polydefkis M. Assessment of epidermal nerve fibers: a new diagnostic and predictive tool for peripheral neuropathies. J Neuropathol Exp Neurol 2007;66:10591073.

10. Smith AG, Howard JR, Kroll R, et al. The reliability of skin biopsy with measurement of intraepidermal nerve fiber density. J Neurol Sci 2005;228:65-69.

11. Hunninghake GW, Costabel U, Ando M, et al. ATS/ERS/WASOG statement on sarcoidosis. American Thoracic Society/European Respiratory Society/World Association of Sarcoidosis and other Granulomatous Disorders. Sarcoidosis Vasc Diffuse Lung Dis 1999;16:149-173.

12. Lauria G, Penza P, Melli G, et al. European inter-laboratoy agreement project on skin biopsy standardization and innervation count. Journal of the Peripheral Nervous System 2007;12:49, 50.

13. Hoitsma E. Small fiber neuropathy. A novel finding in sarcoidosis. Maastricht: University of Maastricht, 2005.

14. Streiner DL, Norman GR. Health measurement scales. A practical guide to their development and use., second edition ed. New York: Oxford University Press, 1998.

15. Ware JE, jr., Snow KK, Kosinski M, Gandek B. SF-36 health survey. Manual and interpretation guide. Boston: The health institue, New England Medical Center, 1993.

16. Vickrey BG, Hays RD, Beckstrand M. Development of a health-related quality of life measure for peripheral neuropathy. Neurorehabil Neural Repair 2000;14:93-104.

17. Ware JE, Jr., Gandek B, Kosinski M, et al. The equivalence of SF-36 summary health scores estimated using standard and country-specific algorithms in 10 countries: results from the IQOLA Project. International Quality of Life Assessment. J Clin Epidemiol 1998;51:1167-1170.

18. Ware JE, Kosinski M, Keller SD. SF-36 physical and mental health summary scales: a user's manual. Boston: The Health Assessment Lab, New England Medical Center, 1994.

19. Jorgensen CK, Karlsmose B. Validation of automated forms processing. A comparison of Teleform with manual data entry. Comput Biol Med 1998;28:659-667.

20. McArthur JC, Stocks EA, Hauer P, Cornblath DR, Griffin JW. Epidermal nerve fiber density: normative reference range and diagnostic efficiency. Arch Neurol 1998;55:1513-1520.

21. Cohen J. Weighted kappa: Nominal scale agreement with provision for scal disagreement or part credit. Psychol Bull 1968;70:213-230

22. Goransson LG, Mellgren SI, Lindal S, Omdal R. The effect of age and gender on epidermal nerve fiber density. Neurology 2004;62:774-777.

23. Chang YC, Lin WM, Hsieh ST. Effects of aging on human skin innervation. Neuroreport 2004;15:149-153.

24. Umapathi T, Tan WL, Tan NC, Chan YH. Determinants of epidermal nerve fiber density in normal individuals. Muscle Nerve 2006;33:742-746.

25. Martina IS, van Koningsveld R, Schmitz PI, van der Meche FG, van Doorn PA. Measuring vibration threshold with a graduated tuning fork in normal aging and in patients with polyneuropathy. European Inflammatory Neuropathy Cause and Treatment (INCAT) group. J Neurol Neurosurg Psychiatry 1998;65:743-747. 
26. Merkies IS, Schmitz PI, Samijn JP, Meche FG, Toyka KV, van Doorn PA. Assessing grip strength in healthy individuals and patients with immune-mediated polyneuropathies. Muscle Nerve 2000;23:1393-1401.

27. Lin YH, Hsieh SC, Chao CC, Chang YC, Hsieh ST. Influence of aging on thermal and vibratory thresholds of quantitative sensory testing. J Peripher Nerv Syst 2005;10:269-281.

28. Rivner MH, Swift TR, Malik K. Influence of age and height on nerve conduction. Muscle Nerve 2001;24:1134-1141.

29. Meh D, Denislic M. Quantitative assessment of thermal and pain sensitivity. J Neurol Sci 1994;127:164169.

30. Jain S, Muzzafarullah S, Peri S, Ellanti R, Moorthy K, Nath I. Lower touch sensibility in the extremities of healthy Indians: further deterioration with age. J Peripher Nerv Syst 2008;13:47-53.

31. Bolton CF, Winkelmann RK, Dyck PJ. A quantitative study of Meissner's corpuscles in man. Neurology 1966;16:1-9.

32. Bruce MF, Sinclair DC. The relationship between tactile thresholds and histology in the human finger. J Neurol Neurosurg Psychiatry 1980;43:235-242

33. Roglio I, Giatti S, Pesaresi M, et al. Neuroactive steroids and peripheral neuropathy. Brain Res Rev 2008;57:460-469.

34. Koenig HL, Gong WH, Pelissier P. Role of progesterone in peripheral nerve repair. Rev Reprod 2000;5:189199.

35. Kovacic U, Sketelj J, Bajrovic FF. Sex-related difference in collateral sprouting of nociceptive axons after peripheral nerve injury in the rat. Exp Neurol 2003;184:479-488.

36. Devigili G, Tugnoli V, Penza P, et al. The diagnostic criteria for small fibre neuropathy: from symptoms to neuropathology. Brain 2008;131:1912-1925

37. Scherens A, Maier C, Haussleiter IS, et al. Painful or painless lower limb dysesthesias are highly predictive of peripheral neuropathy: comparison of different diagnostic modalities. Eur J Pain 2009;13:711-718.

38. Obermann M, Katsarava Z, Esser S, et al. Correlation of epidermal nerve fiber density with pain-related evoked potentials in HIV neuropathy. Pain 2008;138:79-86.

39. Merkies IS, Schmitz PI, van der Meche FG, Samijn JP, van Doorn PA. Connecting impairment, disability, and handicap in immune mediated polyneuropathies. J Neurol Neurosurg Psychiatry 2003;74:99-104.

40. Merkies IS, Lauria G. 131st ENMC international workshop: selection of outcome measures for peripheral neuropathy clinical trials 10-12 December 2004, Naarden, The Netherlands. Neuromuscul Disord 2006;16:149-156. 
Chapter

2.1 



\section{INTRAEPIDERMAL NERVE} FIBER DENSITY AT THE

DISTAL LEG: A WORLDWIDE REFERENCE STUDY

G. Lauria', M. Bakkers', C. Schmitz³, R. Lombardi', P. Penza', G. Devigili', A.G. Smith5, S-THsieh', S.I. Mellgren', T. Umapathi', D. Ziegler', C.G. Faber', I.S.J. Merkies',10

'Neuromuscular Diseases Unit, IRCCS Foundation "Carlo Besta" Neurological Institute, Milan, Italy

2Department of Neurology, Maastricht University Medical Centre, Maastricht, the Netherlands ${ }^{3}$ School of Mental Health and Neuroscience, Maastricht University, Maastricht, the Netherlands ${ }^{4}$ Neurologic Clinic, University of Ferrara, Italy ${ }^{5}$ Department of Neurology, University of Utah, Salt Lake City, UT, USA ${ }^{6}$ Department of Neurology, National Taiwan University College of Medicine and National Taiwan University Hospital, Taipei, Taiwan 7 Department of Clinical Medicine (Neurology), University of Tromsø, Norway

${ }^{8}$ National Neuroscience Institute, Singapore 9 Institute for Clinical Diabetology, German Diabetes Center at the Heinrich Heine University, Leibniz Center for Diabetes Research, Department of Metabolic Diseases, University Hospital, Düsseldorf, Germany ${ }^{10}$ Department of Neurology, Spaarne Hospital, Hoofddorp, the Netherlands 


\section{ABSTRACT}

The diagnostic reliability of skin biopsy in small fiber neuropathy depends on the availability of normative reference values. We performed a multicenter study to assess the normative values of intraepidermal nerve fiber (IENF) density at distal leg stratified by age deciles. Eight skin biopsy laboratories from Europe, USA, and Asia submitted eligible data. Inclusion criteria of raw data were healthy subjects 18 years or older; known age and gender; 3-mm skin biopsy performed 10-cm above the lateral malleolus; bright-field immunohistochemistry protocol, and quantification of linear IENF density in three $50-\mu \mathrm{m}$ sections according to published guidelines. Data on height and weight were recorded, and body mass index (BMI) was calculated in subjects with both available data. Normative IENF density reference values were calculated through quantile regression analysis; influence of height, weight, or BMI was determined by regression analyses. IENF densities from 550 participants (285 women, 265 men) were pooled. We found a significant age-dependent decrease of IENF density in both genders (women $p<0.001$; men $p=0.002$ ). Height, weight, or BMI did not influence the calculated 5th percentile IENF normative densities in both genders. Our study provides IENF density normative reference values at the distal leg to be used in clinical practice. 


\section{INTRODUCTION}

Skin punch biopsy with measurement of intraepidermal nerve fiber (IENF) density has become a valuable tool in the evaluation of patients with neuropathy. For some patients, especially when small nerve fibers appear to be predominantly affected, this may be the only test that objectively shows abnormalities and thus confirms the diagnosis of small fiber neuropathy (SFN)., 2 Although skin biopsy with IENF quantification has become standard and many laboratories have reported their own normal values, questions remain. First, two different techniques are used (e.g., bright-field immunohistochemistry and indirect immunofluorescence with or without confocal microscopy), which diagnostic yield has not been compared so far. Second, and most important, the effect of anthropomorphic and demographic parameters, including age, height, weight, and body mass index (BMI) on IENF density values is unknown and stratified normative data are lacking. Indeed, most articles reported the difference between cohorts of patients and healthy subjects in terms of mean, median, or cut-off values.-20 Some have demonstrated differences between men and women, a decline of IENF density with increasing age, or confounding influence of height and weight. ${ }^{3,21-23}$ More recently, some of us reported the results of a multicenter study showing age and gender-dependent IENF density values in healthy subjects. ${ }^{24}$ Because it is debated whether these values are representative for other centers, we aimed to calculate the normative reference values of IENF density at the distal leg through a worldwide multicenter collaboration in order to support a general standardized use of skin biopsy in the diagnosis of SFN.

\section{MATERIALS AND METHODS}

\section{Participants}

Nine groups who previously reported normative IENF density values at the distal leg using bright-field immunohistochemistry were approached for collaboration. They were invited to provide the coordinating center (Milan, Italy) with their available IENF density data for healthy subjects. Eight centers decided to participate in the project. Eligibility criteria for inclusion of raw data were: (1) healthy subjects 18 years or older, free of neurological signs and symptoms, and conditions known to be at risk for neuropathy; (2) known age and gender; (3) specimens obtained through 3-mm punch skin biopsy $10 \mathrm{~cm}$ above the lateral malleolus, in the territory of the sural nerve; (4) use of bright-field immunohistochemistry protocol according to published guidelines; ${ }^{25}$ (5) quantification of linear IENF density (number of fibers/millimeter) in at least three sections of 50- $\mu \mathrm{m}$ thickness according to published counting rules (IENF have to cross or originate at the dermal-epidermal junction, and secondary branches and fragments are not counted). ${ }^{26}$ Data on height and weight were recorded when available, and 
body mass index $(\mathrm{BMI}=$ weight $[\mathrm{kg}] /$ height $[\mathrm{cm}] \times$ height $[\mathrm{cm}])$ was calculated in subjects with both available data.

\section{Statistical analysis}

Normative IENF density reference values were then calculated through quantile regression analysis (Gould and Rogers, 1994). ${ }^{27}$ Possible influence of height and weight (or BMI) on IENF density in men and women was determined by regression studies. The median values and chosen cut-off at the 5 th percentile are presented for men and women per age decade.

\section{RESULTS}

Eight centers decided to participate in the study and provided data on 550 healthy subjects (women: $n=285$, age: mean 44.9 years [SD 15.2], range 19-92 years; men: $n=265$, age: mean 49.0 [SD 15.8], range 18-84 years). There was a significant agedependent decrease of IENF density values in both genders (in women: $p<0.001$; in men: $p=0.002$ ). Up to 70 years, the normative IENFD findings were lower in men compared to women, but became equivalent in the older age groups (70 years plus) (Figure 1 and Table 1).

Figure 1. IEFD of healthy individuals (285 women, 265 men).
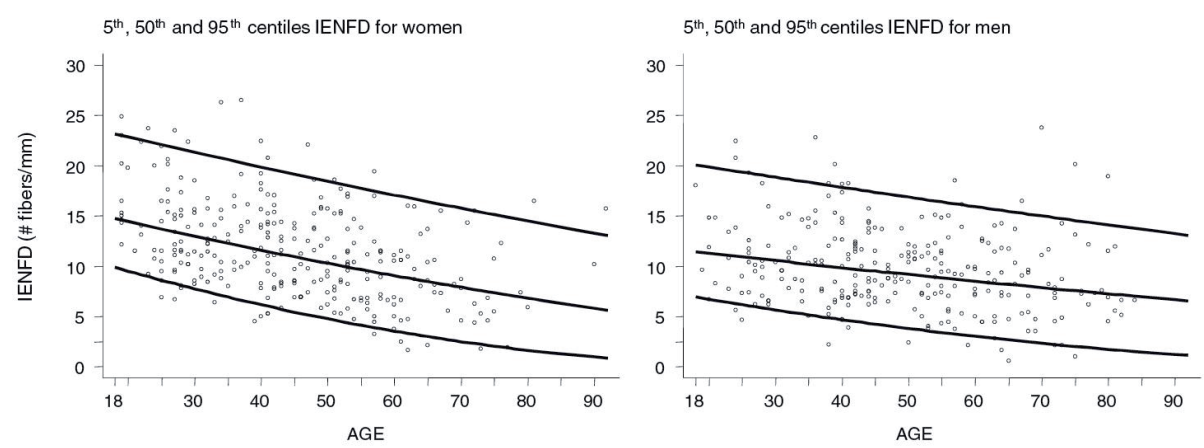

Scatter plot showing normative intra epidermal nerve fiber density (IENFD) scores in healthy individuals stratified for age and gender (lines depict $5^{\text {th }}, 50^{\text {th }}$ and $95^{\text {th }}$ centiles).

Data on height were available from 320 subjects (161 women and 159 men) and on weight from 247 subjects (117 women and 130 men). Women had a mean height of $163 \mathrm{~cm}$ (SD 10; range 141-198) and men of $173 \mathrm{~cm}$ (SD 9; range 150-196). Women had a mean weight of $65.3 \mathrm{~kg}$ (SD 13.8; range 40-132) and men of $75.6 \mathrm{~kg}$ (SD 16.8; range 48.6-164). Multivariate regression analysis did not show any significant impact of weight on IENF density in women, whereas in men there was a minor but significant 
inverse correlation that explained a proportion of $12 \%$ of IENF density findings $\left(R^{2}=\right.$ $0.12 ; \mathrm{p}<0.001)$. The BMI values demonstrated similar findings: in women (mean 24.1 \pm 5.0 SD), where it explained only $3 \%\left(R^{2}=0.028 ; p=0.02\right)$ and in men (mean $24.7 \pm$ 4.4 SD), where it explained $13 \%\left(R^{2}=0.13 ; p=0.001\right)$ of IENF density findings. Height did not influence IENF densities in men or women. However, for the calculated IENF density normative scores (5th percentile values), no influence of height, weight, or BMI was seen in both genders.

Table 1. Intra-epidermal nerve fiber density (IENFD) normative values for clinical use.

\begin{tabular}{c|ccc|ccc}
\hline \multirow{2}{*}{ Age (years) } & \multicolumn{3}{|c|}{ Females (97) } & \multicolumn{3}{c}{ Males (91) } \\
\cline { 2 - 7 } & $\begin{array}{c}\text { Number of } \\
\text { subjects }\end{array}$ & $\begin{array}{l}\text { 0.05 quantile } \\
\text { IENFD values } \\
\text { per age span }\end{array}$ & $\begin{array}{c}\text { median } \\
\text { IENFD values } \\
\text { per age span }\end{array}$ & $\begin{array}{c}\text { Number of } \\
\text { subjects }\end{array}$ & $\begin{array}{c}\mathbf{0 . 0 5} \text { quantile } \\
\text { IENFD values } \\
\text { per age span }\end{array}$ & $\begin{array}{c}\text { median } \\
\text { IENFD values } \\
\text { per age span }\end{array}$ \\
\hline $20-29$ & 57 & 8.4 & 13.5 & 36 & 6.1 & 10.9 \\
$30-39$ & 47 & 7.1 & 12.4 & 40 & 5.2 & 10.3 \\
$40-49$ & 70 & 5.7 & 11.2 & 62 & 4.4 & 9.6 \\
$50-59$ & 59 & 4.3 & 9.8 & 53 & 3.5 & 8.9 \\
$60-69$ & 32 & 3.2 & 8.7 & 43 & 2.8 & 8.3 \\
$70-79$ & 16 & 2.2 & 7.6 & 22 & 2.1 & 7.7 \\
$\geq 80$ & 4 & 1.6 & 6.7 & 9 & 1.7 & 7.2 \\
\hline
\end{tabular}

\section{DISCUSSION}

This report presents the pooled normative IENF density values at the distal leg from eight skin biopsy laboratories. The findings demonstrated that IENF density gradually declines with increasing age. Up to 70 years, the normative values were higher in women compared to men. In the higher age groups (70 years plus), the normative values became quite equivalent between the two genders. Moreover, we found that height does not influence IENF densities, whereas weight and BMI had a small inverse correlation in men. However, none of these parameters (height, weight, or BMI) demonstrated a significant impact on the 5th percentile IENF normative densities in both genders. Therefore, the 5 th percentile values provided can be used in clinical practice without adjustment to these anthropomorphic parameters.

These results confirm previous observations (Table 2) and definitely establish the normative reference values of IENF density at the distal leg to be used in clinical practice and research. Data originate from the largest sample size ever analyzed with the same methods, and are valid for bright-field immunohistochemistry using specific counting rules, which exclude IENF fragments from quantification. Fragments have been formerly included in the quantification of IENF density. ${ }^{28}$ Whether different counting rules would give different cut-off values needs to be investigated by a focused comparative study including healthy subjects and neuropathy patients. 
The decrease of IENF density values with age is in line with studies reporting an agerelated decline of other direct or indirect measures of neurological functions, such as grip strength, sensory testing, sensory nerve action potential amplitude, and Meissner and Pacinian corpuscle density. ${ }^{29-33}$ However, the correlation between age and loss of unmyelinated axons in human beings is controversial. ${ }^{34}$ The reasons for the agerelated decline in IENF density are likely related to the physiological processes of aging. The differences in epidermal innervation density between men and women may be related to gender-related hormonal status. ${ }^{33}$ It has been shown that progesterone stimulates axonal growth and myelination, and may have a neuroprotective effect on IENF. Furthermore, nerve sprouting, regeneration, and remyelination have been demonstrated to occur at a more rapid speed in female rats. ${ }^{35,36}$

Skin biopsy with quantification of IENF density at the distal leg is considered a reliable tool to confirm the clinical diagnosis of SFN. ${ }^{1,2}$ Although conclusive diagnostic criteria for SFN are not yet available, most authors used a similar definition based on normal sural nerve conduction study, clinical symptoms and signs considered suggestive, and altered skin biopsy or quantitative sensory testing (QST) findings. 5, 24, 37-48 However, in most clinical series patients were compared to normative mean or cut-off values of IENF density obtained from nonstratified control groups. These values are higher than the age-related normative values, possibly leading to an overdiagnosis of SFN. The availability of cut-off values stratified per age decade and gender will allow the definition of the clinical diagnosis of SFN with a higher certainty.

The 5th percentile cut-off values in this study were only slightly higher than those recently reported, with comparable age-related decrease of IENF densities, even though the subjects were not exactly stratified as it was presented previously. ${ }^{24}$ However, there are still some unresolved issues regarding the reliability of normative IENF density values. The 5 th percentile was used as cut-off value in previous works. ${ }^{24,28}$ In a recent paper, Nebuchennykh and colleagues compared the diagnostic yield of skin biopsy at the distal leg in 45 patients with SFN and 134 healthy subjects using three statistical methods: (1) Z-scores, calculated from multiple regression analysis, which cut-off values were estimated for each patient and adjusted for age and gender; (2) 5th percentile with $6.7 \mathrm{IENF} / \mathrm{mm}$ cut-off value; and (3) receiver operating characteristic (ROC) analysis with a cut-off value of $10.3 \mathrm{IENF} / \mathrm{mm} .^{48}$ Z-scores and 5 th percentile showed higher specificity ( $98 \%$ and $95 \%$, respectively) but lower sensitivity (31\% and $35 \%$, respectively) compared to the ROC analysis that showed specificity of $64 \%$ and sensitivity of $78 \%$. We agree that the diagnostic yield of skin biopsy may depend on how the reference and cut-off values have been assessed and on the diagnostic criteria for SFN which still need to be defined. Further studies are warranted to establish specificity and sensitivity values of IENF density using the reference normative values provided by this work, which we consider valid for clinical practice use. 


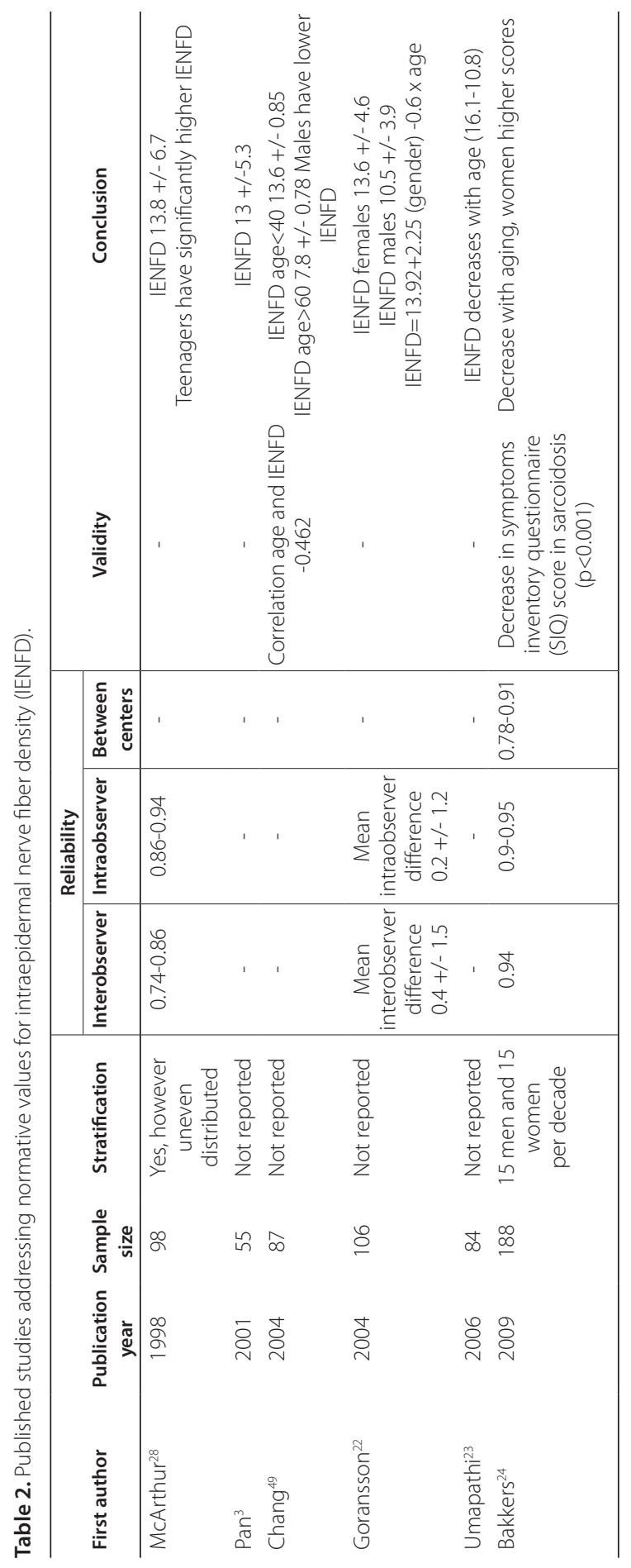

Chapter

2.2 


\section{REFERENCES}

1. England JD, Gronseth GS, Franklin G, et al. Evaluation of distal symmetric polyneuropathy: the role of autonomic testing, nerve biopsy, and skin biopsy (an evidence-based review). Muscle Nerve 2009;39:106-115.

2. Lauria G, Hsieh ST, Johansson O, et al. European Federation of Neurological Societies/Peripheral Nerve Society Guideline on the use of skin biopsy in the diagnosis of small fiber neuropathy. Report of a joint task force of the European Federation of Neurological Societies and the Peripheral Nerve Society. Eur $J$ Neurol 2010;17:903-912, e944-909.

3. Pan CL, Lin YH, Lin WM, TaiTY, Hsieh ST. Degeneration of nociceptive nerve terminals in human peripheral neuropathy. Neuroreport 2001;12:787-792.

4. Kennedy WR, Wendelschafer-Crabb G, Johnson T. Quantitation of epidermal nerves in diabetic neuropathy. Neurology 1996;47:1042-1048.

5. Holland NR, Crawford TO, Hauer P, Cornblath DR, Griffin JW, MCArthur JC. Small-fiber sensory neuropathies: clinical course and neuropathology of idiopathic cases. Ann Neurol 1998;44:47-59.

6. Herrmann DN, Griffin JW, Hauer P, Cornblath DR, McArthur JC. Epidermal nerve fiber density and sural nerve morphometry in peripheral neuropathies. Neurology 1999;53:1634-1640.

7. Periquet MI, Novak V, Collins MP, et al. Painful sensory neuropathy: prospective evaluation using skin biopsy. Neurology 1999;53:1641-1647.

8. Nolano M, Provitera V, Crisci C, et al. Small fibers involvement in Friedreich's ataxia. Ann Neurol 2001;50:1725.

9. Pan CL, Tseng TJ, Lin YH, Chiang MC, Lin WM, Hsieh ST. Cutaneous innervation in Guillain-Barre syndrome: pathology and clinical correlations. Brain 2003;126:386-397.

10. Chiang MC, Lin YH, Pan CL, Tseng TJ, Lin WM, Hsieh ST. Cutaneous innervation in chronic inflammatory demyelinating polyneuropathy. Neurology 2002;59:1094-1098.

11. Hoitsma E, Marziniak M, Faber CG, et al. Small fibre neuropathy in sarcoidosis. Lancet 2002;359:20852086.

12. Polydefkis M, Yiannoutsos CT, Cohen BA, et al. Reduced intraepidermal nerve fiber density in HIVassociated sensory neuropathy. Neurology 2002;58:115-119.

13. Lauria $G$, Morbin $M$, Lombardi $R$, et al. Axonal swellings predict the degeneration of epidermal nerve fibers in painful neuropathies. Neurology 2003;61:631-636.

14. Pittenger GL, Ray M, Burcus NI, McNulty P, Basta B, Vinik Al. Intraepidermal nerve fibers are indicators of small-fiber neuropathy in both diabetic and nondiabetic patients. Diabetes Care 2004;27:1974-1979.

15. Goransson LG, Herigstad A, Tjensvoll AB, Harboe E, Mellgren SI, Omdal R. Peripheral neuropathy in primary sjogren syndrome: a population-based study. Arch Neurol 2006;63:1612-1615.

16. Goransson LG, Tjensvoll AB, Herigstad A, Mellgren SI, Omdal R. Small-diameter nerve fiber neuropathy in systemic lupus erythematosus. Arch Neurol 2006;63:401-404.

17. Loseth S, Lindal S, Stalberg E, Mellgren SI. Intraepidermal nerve fibre density, quantitative sensory testing and nerve conduction studies in a patient material with symptoms and signs of sensory polyneuropathy. Eur J Neurol 2006;13:105-111.

18. Loseth S, Stalberg E, Jorde R, Mellgren SI. Early diabetic neuropathy: thermal thresholds and intraepidermal nerve fibre density in patients with normal nerve conduction studies. J Neurol 2008;255:1197-1202.

19. Sorensen L, Molyneaux L, Yue DK. The relationship among pain, sensory loss, and small nerve fibers in diabetes. Diabetes Care 2006;29:883-887.

20. Gorson KC, Herrmann DN, Thiagarajan R, et al. Non-length dependent small fibre neuropathy/ ganglionopathy. J Neurol Neurosurg Psychiatry 2008;79:163-169.

21. Chien HF, Tseng TJ, Lin WM, et al. Quantitative pathology of cutaneous nerve terminal degeneration in the human skin. Acta Neuropathol 2001;102:455-461.

22. Goransson LG, Mellgren SI, Lindal S, Omdal R. The effect of age and gender on epidermal nerve fiber density. Neurology 2004;62:774-777.

23. Umapathi T, Tan WL, Tan NC, Chan YH. Determinants of epidermal nerve fiber density in normal individuals. Muscle Nerve 2006;33:742-746. 
24. Bakkers M, Merkies IS, Lauria G, et al. Intraepidermal nerve fiber density and its application in sarcoidosis. Neurology 2009;73:1142-1148.

25. Lauria G, Cornblath DR, Johansson O, et al. EFNS guidelines on the use of skin biopsy in the diagnosis of peripheral neuropathy. Eur J Neurol 2005;12:747-758.

26. Kennedy WR, McArthur J, Polydefkis M, Wendelschafer-Crabb G. Pathology and quantitation of cutaneous innervation. In: Thomas PK, Dyck PJ, eds. Peripheral Neuropathy. Philadelphia: Elsevier Saunders, 2005: 869-895.

27. Gould WW, Rogers WH. Quantile regression as an alternative to robust regression. Proceedings of the Statistical Computing Section,. Alexandria, VA: American Statistical Association, 1994

28. McArthur JC, Stocks EA, Hauer P, Cornblath DR, Griffin JW. Epidermal nerve fiber density: normative reference range and diagnostic efficiency. Arch Neurol 1998;55:1513-1520.

29. Merkies IS, Schmitz PI, Samijn JP, Meche FG, Toyka KV, van Doorn PA. Assessing grip strength in healthy individuals and patients with immune-mediated polyneuropathies. Muscle Nerve 2000;23:1393-1401.

30. Bruce MF, Sinclair DC. The relationship between tactile thresholds and histology in the human finger. J Neurol Neurosurg Psychiatry 1980;43:235-242.

31. Martina IS, van Koningsveld R, Schmitz PI, van der Meche FG, van Doorn PA. Measuring vibration threshold with a graduated tuning fork in normal aging and in patients with polyneuropathy. European Inflammatory Neuropathy Cause and Treatment (INCAT) group. J Neurol Neurosurg Psychiatry 1998;65:743-747.

32. Rivner MH, Swift TR, Malik K. Influence of age and height on nerve conduction. Muscle Nerve 2001;24:1134-1141.

33. Roglio I, Giatti S, Pesaresi M, et al. Neuroactive steroids and peripheral neuropathy. Brain Res Rev 2008;57:460-469.

34. Verdu E, Ceballos D, Vilches JJ, Navarro X. Influence of aging on peripheral nerve function and regeneration. J Peripher Nerv Syst 2000;5:191-208.

35. Koenig HL, Gong WH, Pelissier P. Role of progesterone in peripheral nerve repair. Rev Reprod 2000;5:189199.

36. Kovacic U, Sketelj J, Bajrovic FF. Sex-related difference in collateral sprouting of nociceptive axons after peripheral nerve injury in the rat. Exp Neurol 2003;184:479-488.

37. Tavee J, Zhou L. Small fiber neuropathy: A burning problem. Cleve Clin J Med 2009;76:297-305.

38. Goodman BP. Approach to the evaluation of small fiber peripheral neuropathy and disorders of orthostatic intolerance. Semin Neurol 2007;27:347-355.

39. Hoitsma E, Reulen JP, de Baets M, Drent M, Spaans F, Faber CG. Small fiber neuropathy: a common and important clinical disorder. J Neurol Sci 2004;227:119-130.

40. Lacomis D. Small-fiber neuropathy. Muscle Nerve 2002;26:173-188.

41. Mendell JR, Sahenk Z. Clinical practice. Painful sensory neuropathy. N Engl J Med 2003;348:1243-1255.

42. Said G. Small fiber involvement in peripheral neuropathies. Curr Opin Neurol 2003;16:601-602.

43. Sommer C. Painful neuropathies. Curr Opin Neurol 2003;16:623-628.

44. Herrmann DN, Ferguson ML, Pannoni V, Barbano RL, Stanton M, Logigian EL. Plantar nerve AP and skin biopsy in sensory neuropathies with normal routine conduction studies. Neurology 2004;63:879-885.

45. Lauria G. Small fibre neuropathies. Curr Opin Neurol 2005;18:591-597.

46. Sommer C, Lauria G. Chapter 41 Painful small-fiber neuropathies. Handbook of Clinical neurology2006: 621-633.

47. Devigili G, Tugnoli V, Penza P, et al. The diagnostic criteria for small fibre neuropathy: from symptoms to neuropathology. Brain 2008;131:1912-1925.

48. Nebuchennykh M, Loseth S, Lindal S, Mellgren SI. The value of skin biopsy with recording of intraepidermal nerve fiber density and quantitative sensory testing in the assessment of small fiber involvement in patients with different causes of polyneuropathy. J Neurol 2009;256:1067-1075.

49. Chang YC, Lin WM, Hsieh ST. Effects of aging on human skin innervation. Neuroreport 2004;15:149-153. 

Chapter 3.1

\section{TEMPERATURE}

\section{THRESHOLD TESTING:}

\section{A SYSTEMATIC REVIEW}

M. Bakkers', C.G. Faber', M.J.H. Peters', J.P.H. Reulen'2, H. Franssen', T.Z. Fischer, ${ }^{4,5,6}$, I.S.J. Merkies 1,7

'Department of Neurology Maastricht University Medical Center, Maastricht, the Netherlands

²Department of Clinical Neurophysiology, Maastricht University Medical Center,

Maastricht, the Netherlands

${ }^{3}$ Department of Neurophysiology, University Medical Center Utrecht, Utrecht, the Netherlands

${ }^{4}$ Neuroscience Global Clinical Research, Bristol-Myers Squibb, Wallingford, CT, USA

${ }^{5}$ Department of Neurology and Center for Neuroscience and Regeneration Research, Yale

University School of Medicine, New Haven, CT, USA

${ }^{6}$ Rehabilitation Research Center, Veterans Affairs Connecticut Healthcare System, West Haven,

CT, USA ${ }^{7}$ Department of Neurology, Spaarne Hospital, Hoofddorp, the Netherlands 


\section{ABSTRACT}

The diagnosis of small fiber neuropathy (SFN) has been recently defined as typical symptoms due to small nerve fiber dysfunction accompanied by reduced intraepidermal nerve fiber density (IENFD) or abnormal temperature threshold testing (TTT). Guidelines have been published for the assessment of IENFD. However, international guidelines for TTT are lacking. This paper presents a systematic literature review on reported TTT methods and provides recommendations for its future use in studies evaluating patients. A total of 164 papers fulfilled pre-defined requirements and were selected for review. Over 15 types of instruments are currently being used with a variety of methodological approaches for location, stimulus application, and sensation qualities examined. Consensus is needed to standardize the use of TTT as a diagnostic and follow-up tool in patients. 


\section{INTRODUCTION}

To evaluate the sensory nervous system, bedside examination, nerve conduction studies (NCS), and computer-assisted quantitative sensory testing devices are used.' NCS only provide information on large nerve fiber populations. As small nerve fiber function cannot be evaluated with NCS, temperature threshold testing (TTT), determining cold, warm and temperature-induced pain thresholds, has been advocated as a possible tool for diagnosing small fiber neuropathy (SFN). ${ }^{2}$ This paper presents a comprehensive review of reported TTT methods showing great variability in assessing TTT with regard to basic equipment, testing methodology, and site of examination. Recommendations for standardizing the use of TTT are provided.

\section{MATERIALS AND METHODS}

A literature search was conducted to identify all methodological studies published in English in humans that included TTT as a diagnostic or follow-up assessment measure. The search was performed in Pubmed, Medline, Embase, and Cochrane Library engines (period: January 1960 to April 2011). In an attempt to capture all relevant articles, the following pre-defined search terms were used: temperature threshold, thermal threshold, TTT, warm threshold, cold threshold, heat pain, cold pain, quantitative sensory testing (QST). On the basis of title and abstract, a first screening was performed by two authors independently (M. B., M. P.), hereby determining possible relevance of a paper. After reaching consensus, a full paper review and reference tracing was conducted by both authors, independently. In case of discrepancies, the opinion of a third reviewer (C. F.) was requested. Inclusion criteria for review were that the study should report a clear description of test procedure or principle of TTT including the applied testing system, algorithm, settings, condition, and location of testing.

\section{RESULTS}

\section{Search results}

The database search resulted in a total of 2,650 hits. Most papers were excluded because inclusion criteria were not met. After having obtained consensus between the two examiners, a total of 380 articles were selected for full paper review. Reference tracing identified another 85 papers. Of these 465 papers, a total of 301 were subsequently excluded due to limited or no evaluation of TTT and not fulfilling inclusion criteria, 149 papers were included in the final review. 


\section{TTT reported normative values}

The reported TTT normative values for the various commercially available devices are presented in Table 1. Variable age ranges have been used. Stratification for age and gender was only reported in 26\%. ${ }^{3-10}$ An age category was excluded by some researchers, ${ }^{11}$ while others dichotomized their findings by age. ${ }^{12,13}$ The number of healthy subjects included per study varied also considerably from $26^{14}$ to $484 .{ }^{15}$ Only one study compared two devices and showed good correlation between the obtained results. ${ }^{16}$

\section{Testing algorithms}

Multiple testing algorithms have been used in the various studies to determine the TTT findings. These algorithms are classified as being reaction time-independent or reaction time-dependent (Table 1). The reaction time-independent protocols are the so-called "method of levels" or "forced choice" and present a subject with a specific thermode temperature posing the question whether the temperature differs from a baseline temperature or a previously presented temperature. Several protocols exist for the assessment such as the "up and down transform rule", "staircase", or "4-2-1 stepping algorithm". ${ }^{17-19}$ Presenting a trapezoid waveform is generally recommended. ${ }^{20,}$ ${ }^{21}$ For the reaction time-dependent "method of limits", the thermode temperature changes linearly from a baseline temperature and the subject is requested to react by pushing a button as soon as a change in temperature is perceived. Subsequently, the temperature returns to baseline, and this is repeated (in general three to seven times) and averaged to obtain warm, cold, or pain thresholds. The "Marstock method" uses alternating warm and cold stimuli to obtain thresholds for both. ${ }^{14}$ Thresholds are usually expressed in degrees Celsius (absolute temperature sensation threshold or temperature change from starting temperature), or in "just noticeable differences" (CASE IV system). ${ }^{22}$ Moderate to high agreements have been reported between different testing methods. ${ }^{15,23}$ The "method of limits" is less time consuming ${ }^{24,25}$ with similar sensitivity to detect abnormality compared with "method of levels"; 26 however, several studies favor a reaction time-independent method, because reproducibility is better. $^{19,20,25,26}$

\section{Settings variables}

Several variations in the settings of testing algorithms have been reported to influence the findings during TTT evaluation. The reported thermode sizes differed between the various studies. A larger thermode size results in lower thresholds, usually explained by spatial summation. 13, 20, 27-34 The existence of "warmth insensitive fields," skin areas with apparent lack of low threshold warm receptors, may also influence results. ${ }^{34-36}$ A strapped thermode has no significant impact on results, ${ }^{37}$ while varying results are seen when subjects themselves need to apply pressure to the thermode. ${ }^{38}$ A longer stimulus duration in reaction time-independent protocols leads to lower heat pain 
thresholds. ${ }^{39-41}$ An inter-stimulus interval less than a minute may delay the perception of the following stimulus. ${ }^{42}$ Habituation occurs with repeated stimuli, especially when testing heat pain. ${ }^{43}$ The rate of temperature change during testing affects thresholds (higher rate results in higher thresholds) in the reaction time-dependent test algorithm (limits). ${ }^{29,38,39,44-50}$ Higher rates may also increase intra-subject variability. ${ }^{13,40,49,51}$ With temperature change rates under $1^{\circ} \mathrm{C} / \mathrm{s}$, adaptation of warm thresholds occurs. ${ }^{52,53}$ Baseline temperature of the thermode influences the temperature thresholds. For example, a colder thermode $\left(30^{\circ} \mathrm{C}\right)$ leads to better detection of decreasing temperature and a warmer $\left(35^{\circ} \mathrm{C}\right)$ to better detection of increasing temperature, but also increases absolute threshold values. ${ }^{27,51,54,55}$ Limited data exist on the influence of environmental temperatures. However, very cold $\left(<10^{\circ} \mathrm{C}\right)$ or warm $\left(>25^{\circ} \mathrm{C}\right)$ environmental temperatures change skin temperature and warm/cold threshold perception. ${ }^{56}$ When reported, room temperature is mostly kept around $22^{\circ} \mathrm{C}$, with some reports allowing for 10-60 min of acclimatization preceding testing. 11, 39, 50, 55, 57-61 Only few studies have actually changed the skin temperature to study its possible effect on pain ${ }^{39,62}$ and warm and cold detection thresholds, ${ }^{51,56,63}$ with a positive linear relation between skin temperature and warm and cold detection threshold in one study. ${ }^{56}$ Skin temperatures within the range of $25-35^{\circ} \mathrm{C}$ seem of little influence in testing heat pain 39,62 or temperature thresholds. ${ }^{63-68}$ However, an inconsistent pattern has been noted by which skin temperature influences results..$^{59}$ Temperature perception varies with body location, feet being usually less sensitive than hands, 3, 6, 8, 9, 12, 13, 15, 17, 19, 23, 29, 30, 52, 60, $63,64,67,69-77$ but this is not consistently reported. ${ }^{78-80}$ Hairy skin seems more sensitive to pain than glabrous skin, ${ }^{39,78,81}$ but less sensitive for warm and cold detection. ${ }^{63,75}$ Face and especially the lips are most sensitive of all skin areas. ${ }^{10,67,76,77,82,83}$ The tongue is less sensitive. $^{84}$ Left-right differences are insignificant. $^{9,10,12,22,67,75,78,80,82,83,85-89}$

\section{Subject variables}

In addition to device variables that may influence the results, subject variables should also be taken into account. Several studies in adults have reported an increase in temperature thresholds with aging, up to $7^{\circ} \mathrm{C}_{1}^{3,6-9,12,15,19,25,64,65,67,72,77,83,90-96}$ although this is not always a consistent finding., $13,17,57,74,85,87,97-100$ Women are often found to be more sensitive to temperature stimuli than men, $4,9-11,57,66,83,87,88,90,101-104$ but this finding is not always consistently reported. 13, 17, 19,25,73,96, 99, 100, 104, 105 The gender difference is sometimes explained by differences in anthropometric data such as height and weight. ${ }^{8,66,106-108}$ However, stratification for age and gender (in normative studies) was often inadequate (Table 1). Race does not appear to influence temperature detection thresholds, but has been suggested to influence pain experience. ${ }^{109-111}$ TTT is not objective. Results obtained in healthy, paid volunteers may differ from those of laboratory personnel, indicating that cognitive and motivational aspects may be important. ${ }^{76}$ Concentration is essential for reliable results, and the noise of the testing device itself may be a distraction. In repeated testing over time, silence is considered important to keep 
intra-subject variability small. ${ }^{112}$ Sleep deprivation might decrease heat and cold pain thresholds, ${ }^{113}$ although another study showed an effect on mechanical pain thresholds, but not on heat pain. ${ }^{114}$ Cognitive function needs to be intact for best test results, and reduced cognitive function was found to influence cold thresholds. ${ }^{95}$ However, in healthy elderly, cognition was not an important factor in heat threshold detection. ${ }^{57}$ With a proper (reaction time-independent) protocol, even in patients with mental retardation, TTT has been reliably performed. ${ }^{115}$

\section{Reproducibility and responsiveness}

A diagnostic test should be accurate, consistent, and reproducible (Hobart et al. 1996) ${ }^{116}$ and should capture relevant changes (responsive) over time. ${ }^{117}$ Reproducibility of TTT has often been examined with variable results. As few as five healthy subjects ${ }^{65}$ to over 1,100 diabetic patients ${ }^{118}$ have been tested repeatedly, with testing intervals of minutes ${ }^{50,61,82,119,120}$ up to a year, ${ }^{121,122}$ which also contributes to the variations in findings. A comprehensive summary of the reproducibility findings is provided in Table 2. Several studies have calculated the repeatability coefficient, ${ }^{123}$ and showed a wide range from 0.54 to $10.6^{\circ} \mathrm{C} .6,9,19,26,87,96,120,124-127$ Some studies examined reliability by determining the intra-class correlation coefficient (ICC), which varied considerably (ICC: 0.32-0.97). ${ }^{61,87,118,125,128-130}$ Others have reported the coefficient of variation and correlation coefficients, again with a wide range of results. $5,7,24,29,70,73,90,97,118,128,131-137$ Best reported coefficient of variations were under 5\% (warm/heat sensation), but sometimes group means instead of individual values were used, which makes these results less suitable. ${ }^{17,23,126}$ When both warm and cold are tested, usually no large difference is observed between reproducibility of the thresholds between these two modalities. Some studies have shown that warm thresholds have better reproducibility than cold thresholds, ${ }^{7,25,26,85,97}$ while other studies have shown the opposite. ${ }^{61,101,125,129}$ Pain thresholds were more variable than warm and cold thresholds, especially cold pain. 50, 59, 60, 82, 126 Cold pain is also often not studied, as early reports noted absence of cold pain in approximately $15 \%$ of healthy subjects. ${ }^{14,74,85}$ No significant mean change in the temperature thresholds of polyneuropathy subjects occurred after 6-24 months in several studies, ${ }^{138-141}$ though coefficient of variation was reported to be high. ${ }^{97}$ Slight increases of perception thresholds have been found in long-term follow-up (1-2 years) of diabetes patients ${ }^{121,134,142,143}$ and Fabry patients. ${ }^{144}$ Unfortunately, it is unclear if the reported changes are clinically relevant and truly capture responsiveness.

\section{DISCUSSION}

This paper addresses the complexity of TTT examination in clinical practice reported over the last five decades. Great variability in findings exists between studies using a number of devices, different testing algorithms, settings (like variations in thermode 
size, stimulus duration, rate temperature change, baseline temperature) and sites of assessment, and examining various types of sensory qualities in various cohorts of subjects. Reports on normative values differ considerably, likely due to results not stratified for age and gender, varying numbers of subjects examined, both of which hamper clinical applications and utility. Reproducibility has been determined using an equally large array of statistical methods and in different populations with poor to excellent results. There are no studies that examined the responsiveness of TTT in a comprehensive manner. This lack of standardized TTT assessment in clinical practice makes it difficult to compare results found in different studies in the same population of patients. The German Research Network on Neuropathic Pain (DFNS) has compiled a comprehensive protocol for quantitative sensory tests to be used in multicenter studies and has trained participating investigators centrally. ${ }^{83,}{ }^{145}$ However, worldwide standardization has yet to be agreed and initiated.

On the basis of the findings of this review, the following recommendations are made for the use of TTT that could also serve as a frame for a consensus workshop aiming to standardize TTT use:

There should be age and gender stratified normative values.

The reaction time-independent method (method of levels) is suggested, based on no effect of stimulus rate, ${ }^{39,48}$ better reproducibility ${ }^{19,20,23,25,26}$ and applicability in subjects that are difficult to examine such as children and cognitive impaired subjects. ${ }^{59,115}$

As warm and cold sensation are conveyed by different subsets of nerve fibers, ${ }^{146}$ assessment of both is suggested.

Pain assessment is not recommended, because pain thresholds depend on stimulus duration, ${ }^{39-41,47}$ are less reproducible than warm and cold thresholds, ${ }_{1}^{50,59,60,82,126}$ and cold pain is often not perceived. ${ }^{14,74,85}$

The thermode should be strapped to the body ${ }^{37}$ and be large enough to prevent testing warmth insensitive fields. ${ }^{34-36}$

The parameters of the stimuli should be standardized, preferably using a trapezoid waveform. ${ }^{20,21}$

The optimal basal thermode temperature has not been determined, but a temperature approximately $32^{\circ} \mathrm{C}$ is recommended as this is currently most often used.

The subjects should be examined in a quiet, ${ }^{112}$ comfortable temperature controlled room (approximately $22^{\circ} \mathrm{C}$ ). Room temperature need only be corrected if warmer than $25^{\circ} \mathrm{C}$ or cooler than $15^{\circ} \mathrm{C} .56$

Skin temperature should be within the range of $25-35^{\circ} \cdot{ }^{63-67}$

Reproducibility is better at hands than feet, $6,19,26,70,120$ but symptoms often present at feet first. Therefore, assessment at both hands and feet is suggested. However, the clinical value of comparing the various sites of examination should be the focus of future comparative studies.

A comparative study should be performed between the two or three most widely used devices to determine (non-) superiority of one of these devices (Case IV, WR Medical 
Electronics Co., Stillwater, MN, USA; TSA, Medoc, Ramat Yishai, Israel; Thermotest, Somedic, Stockholm, Sweden) and corresponding clinimetric parameters.

In 2003, the American Academy of Neurology concluded that "Quantitative sensory testing (QST) is a potentially useful tool for measuring sensory impairment for clinical and research studies. However, QST results should not be the sole criteria used to diagnose pathology. Because malingering and other non-organic factors can influence the test results, QST is not currently useful for the purpose of resolving medicolegal matters. Well-designed studies comparing different QST devices and methodologies are needed and should include patients with abnormalities detected solely by QST"1.147 To this date, this conclusion still holds its grounds.

In conclusion, the current review highlights the enormous variability found in the assessment of TTT and provides a series of recommendations to improve its future use. 


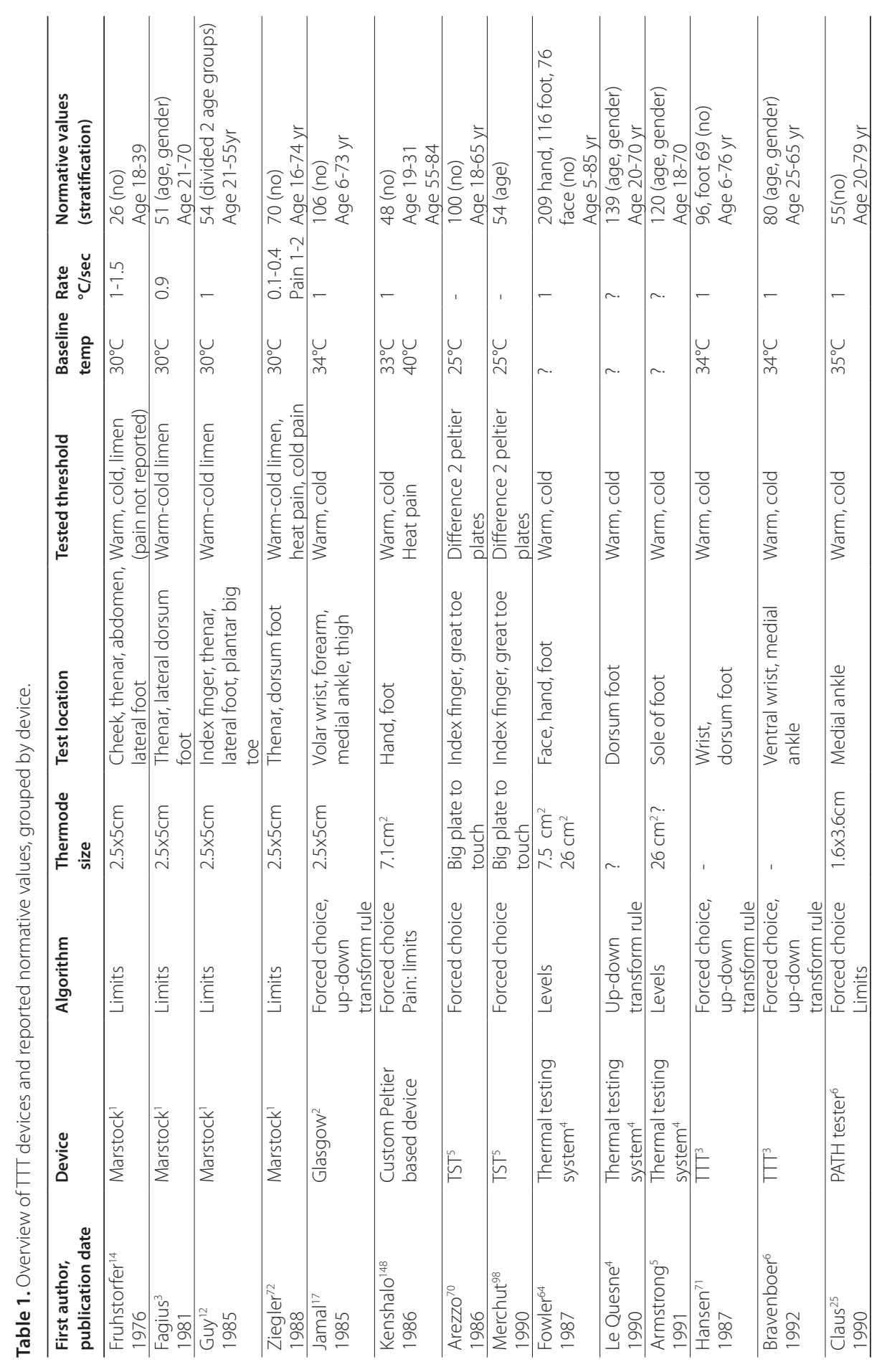

Chapter

3.1 


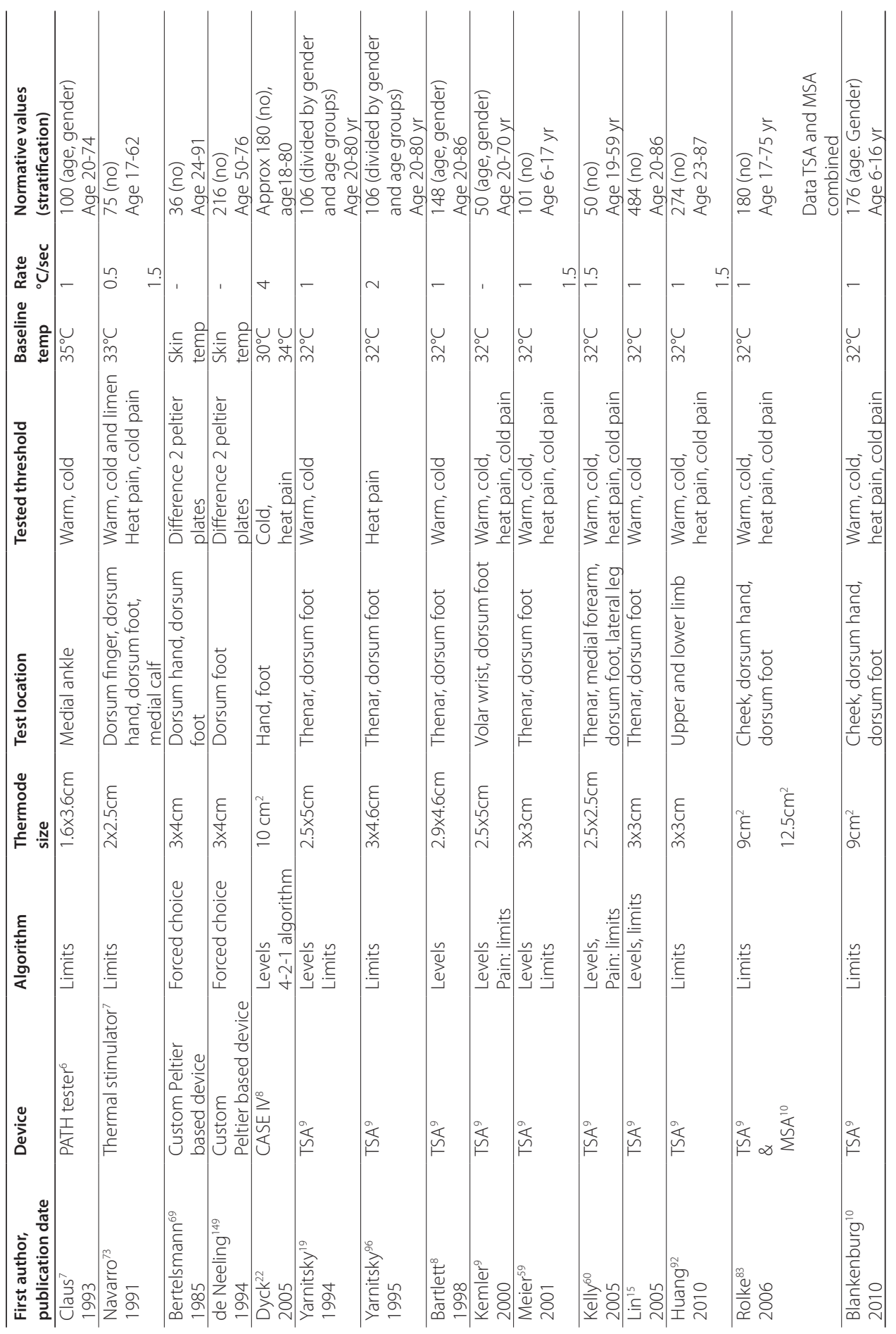




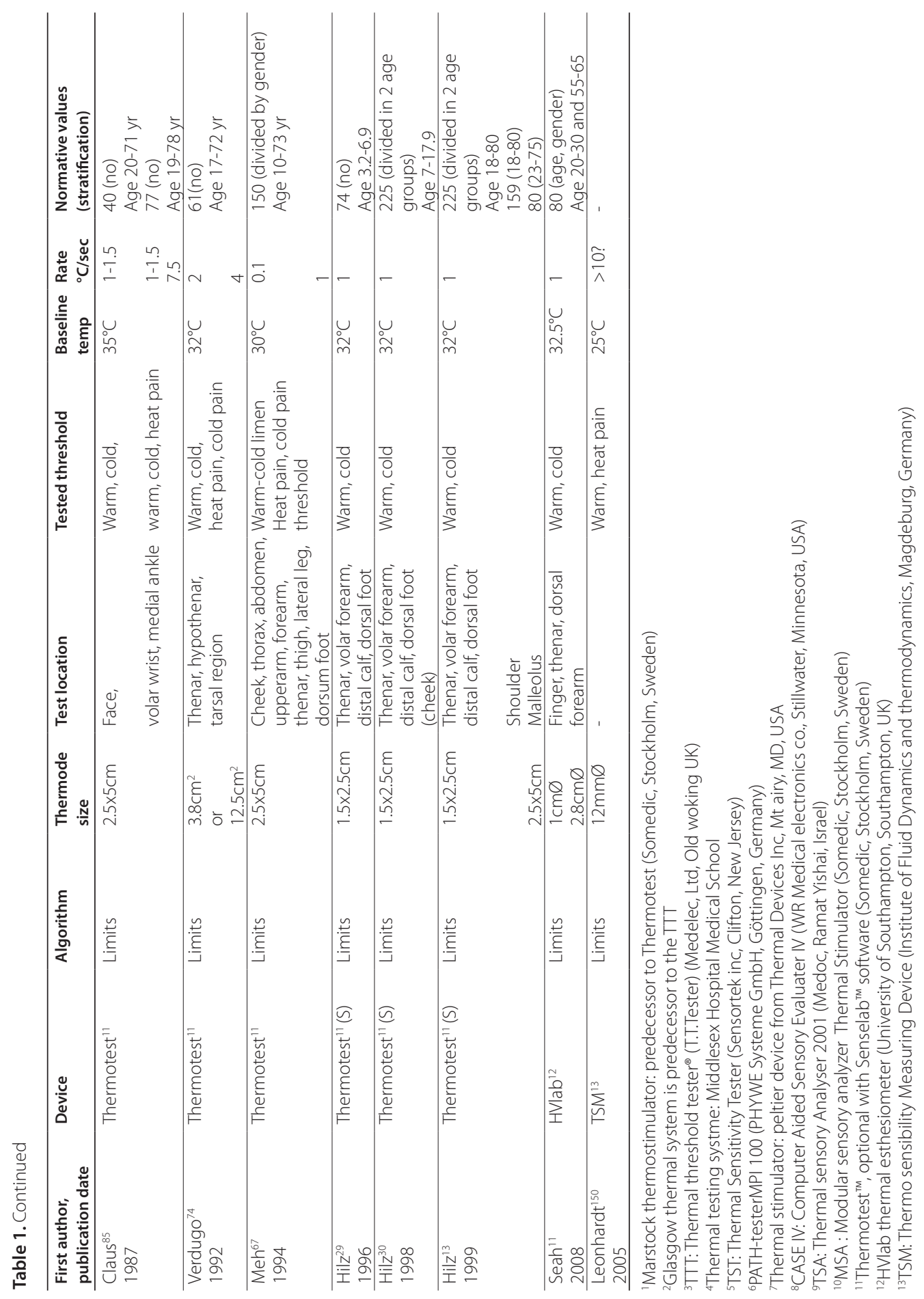

Chapter

3.1 


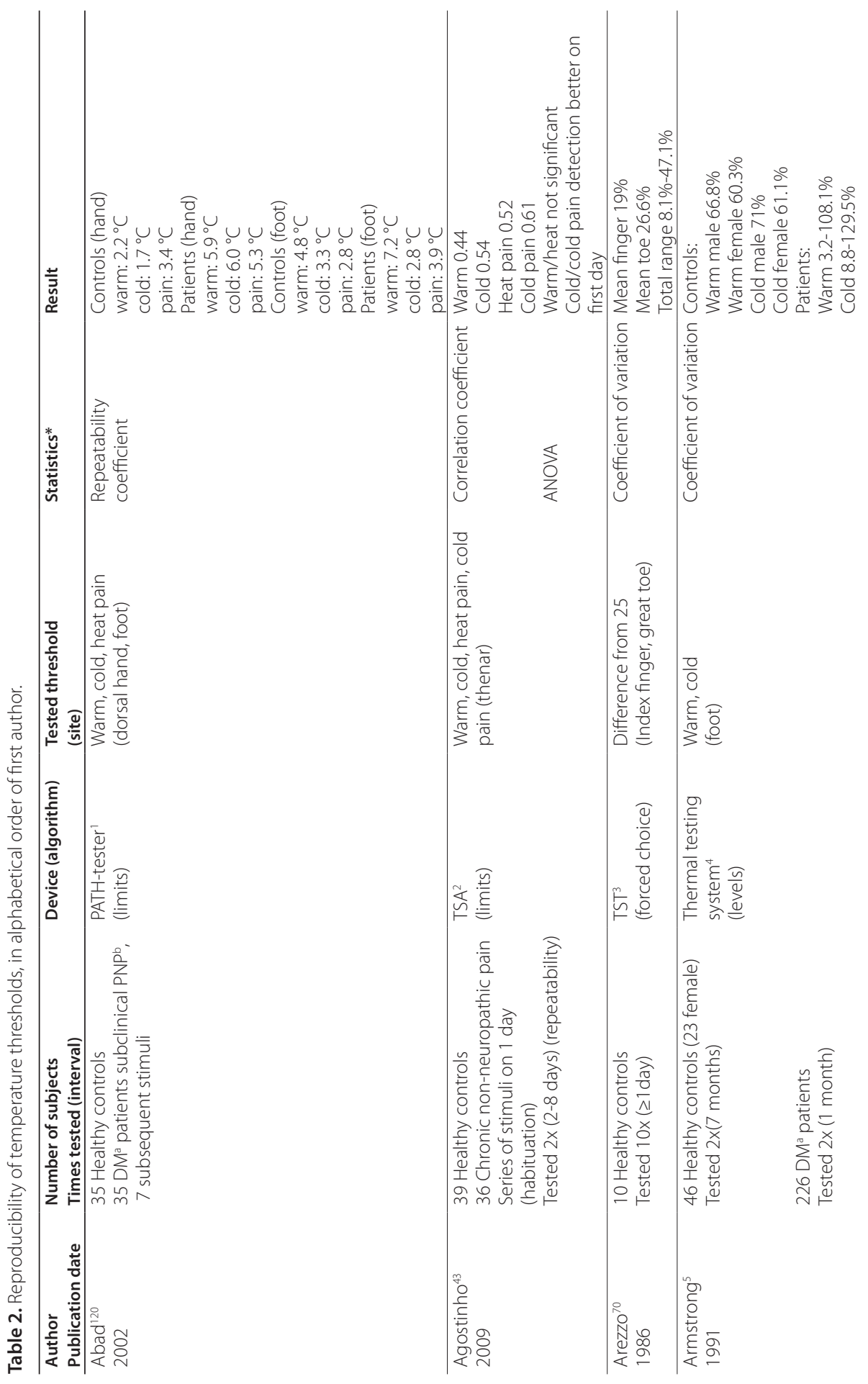




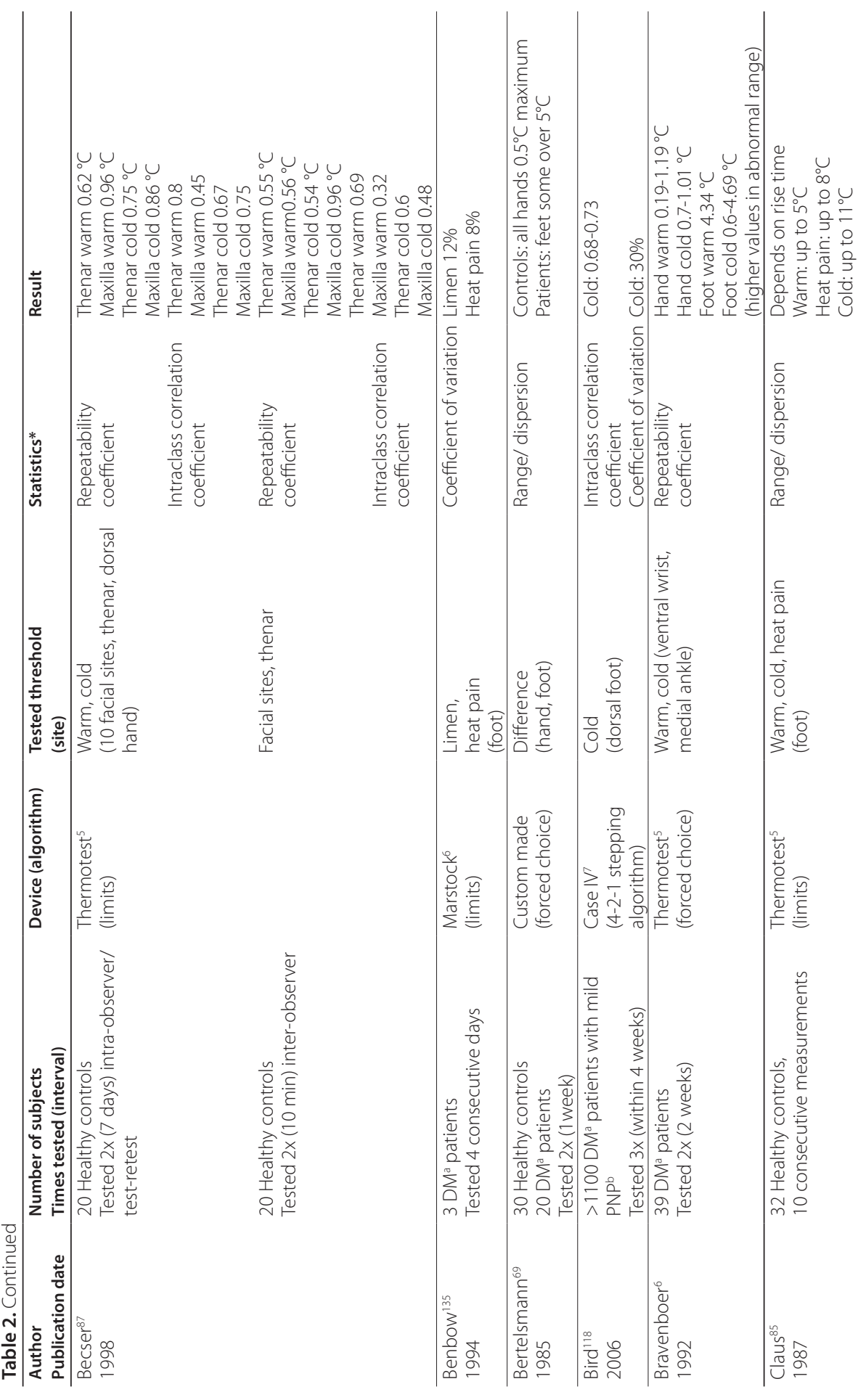

Chapter

3.1 


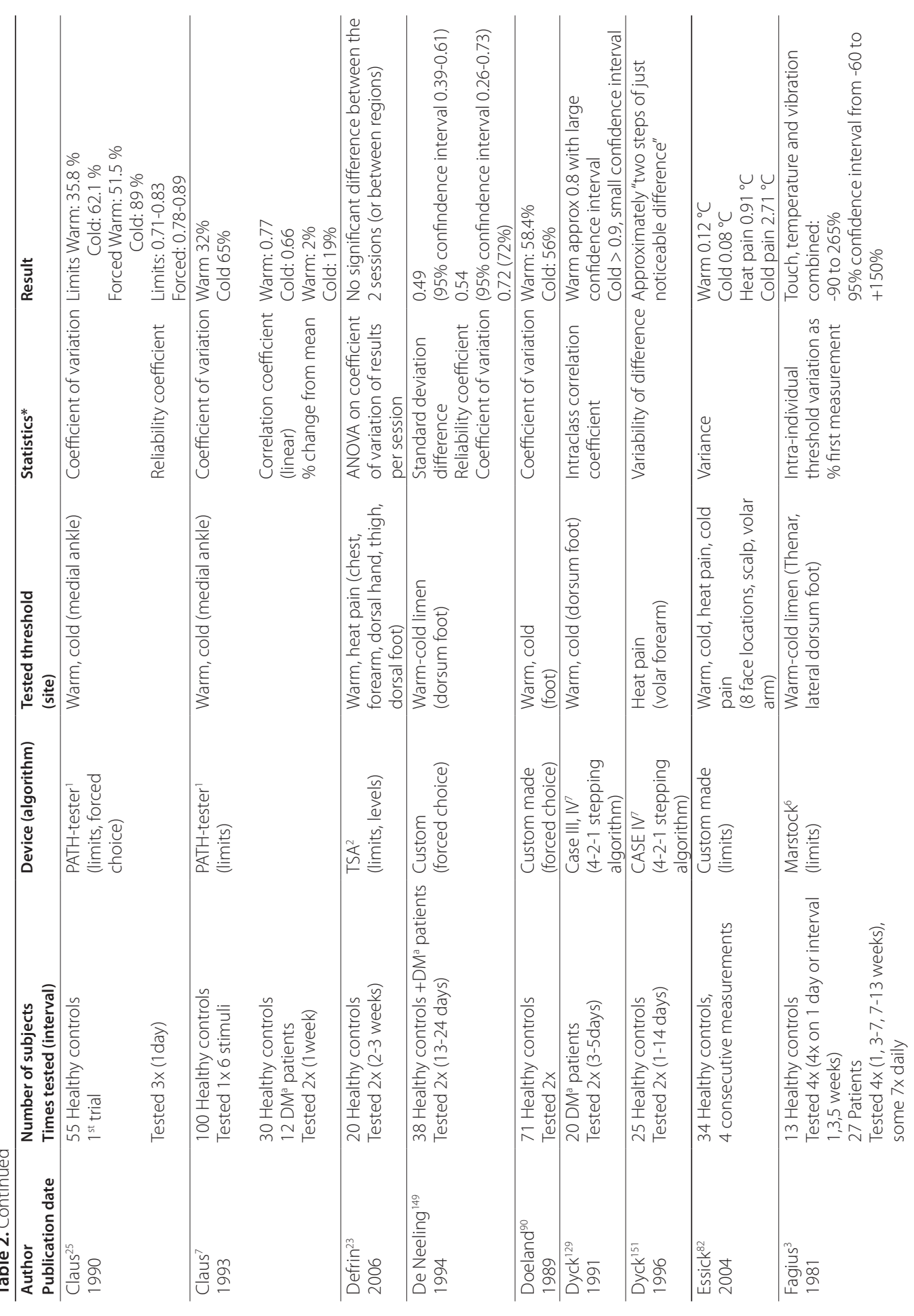




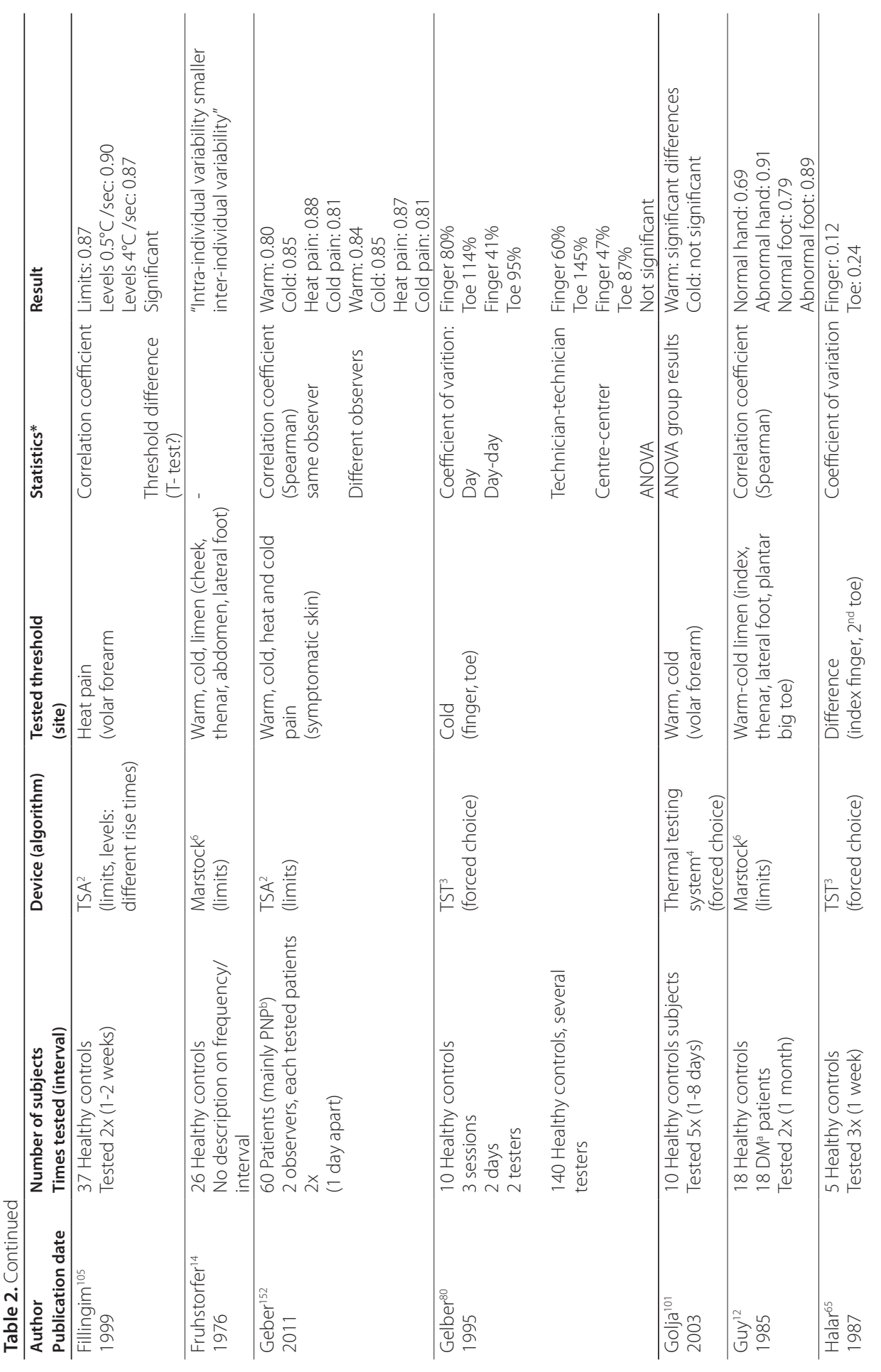

Chapter

3.1 


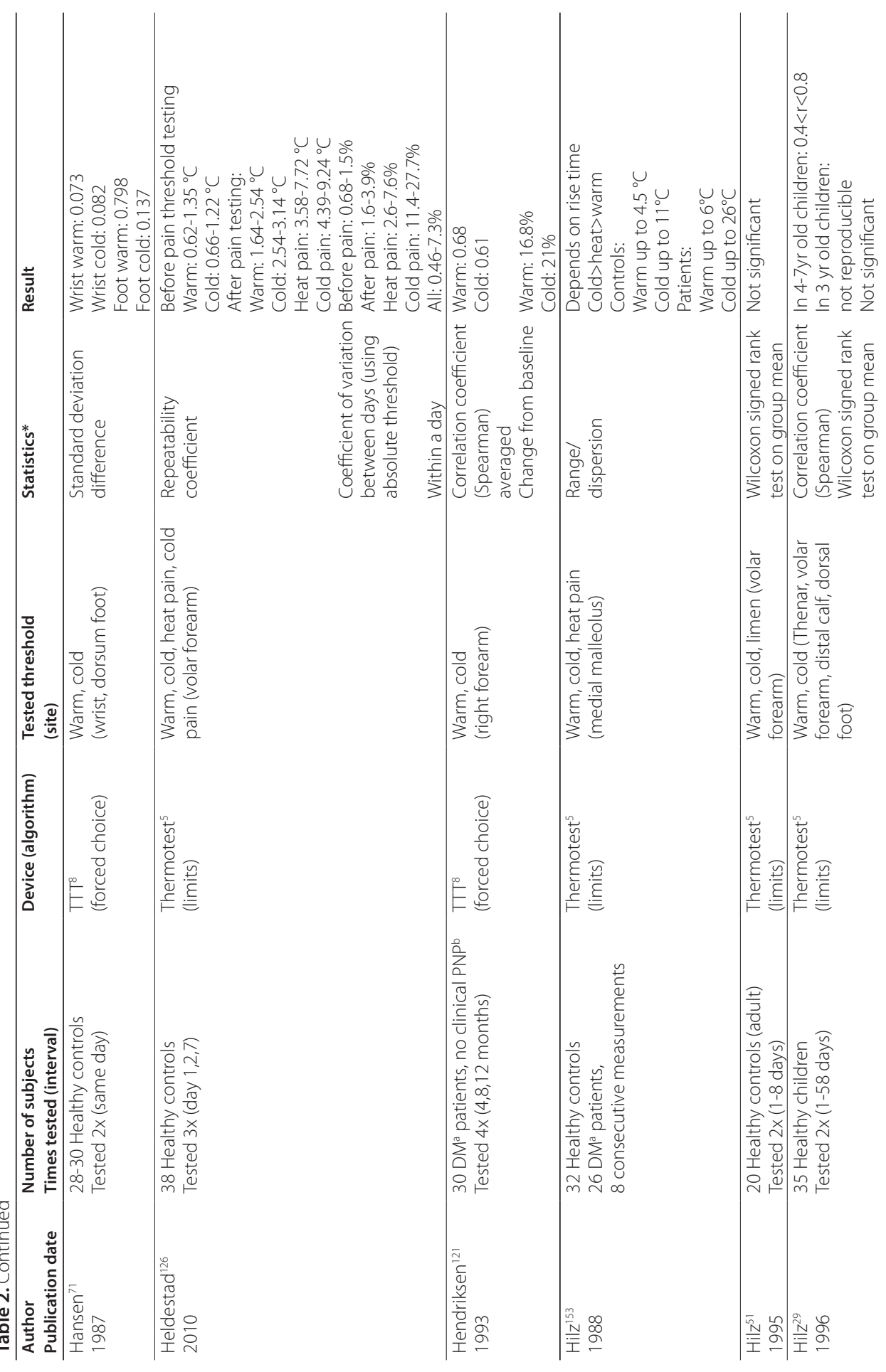




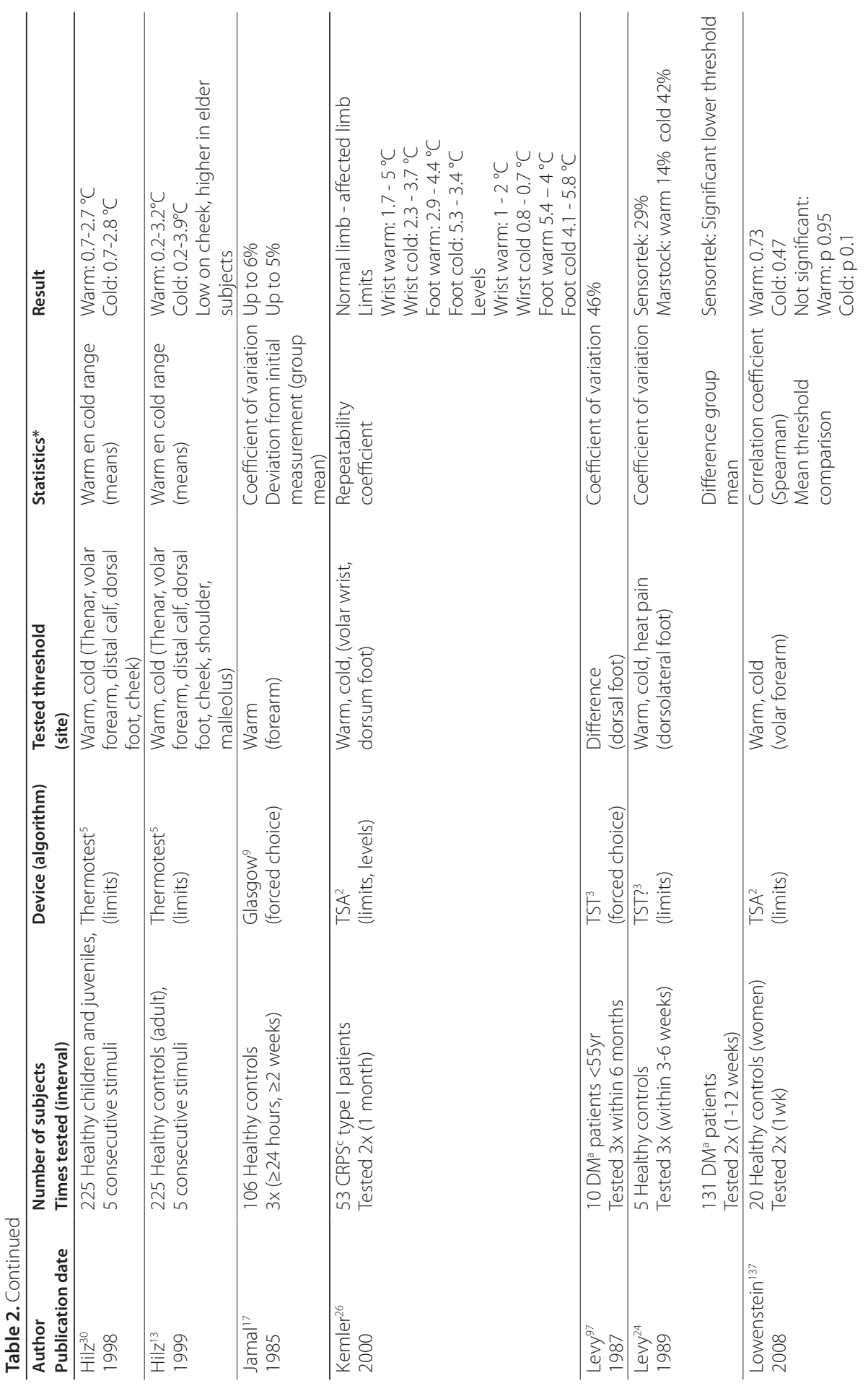

Chapter

3.1 


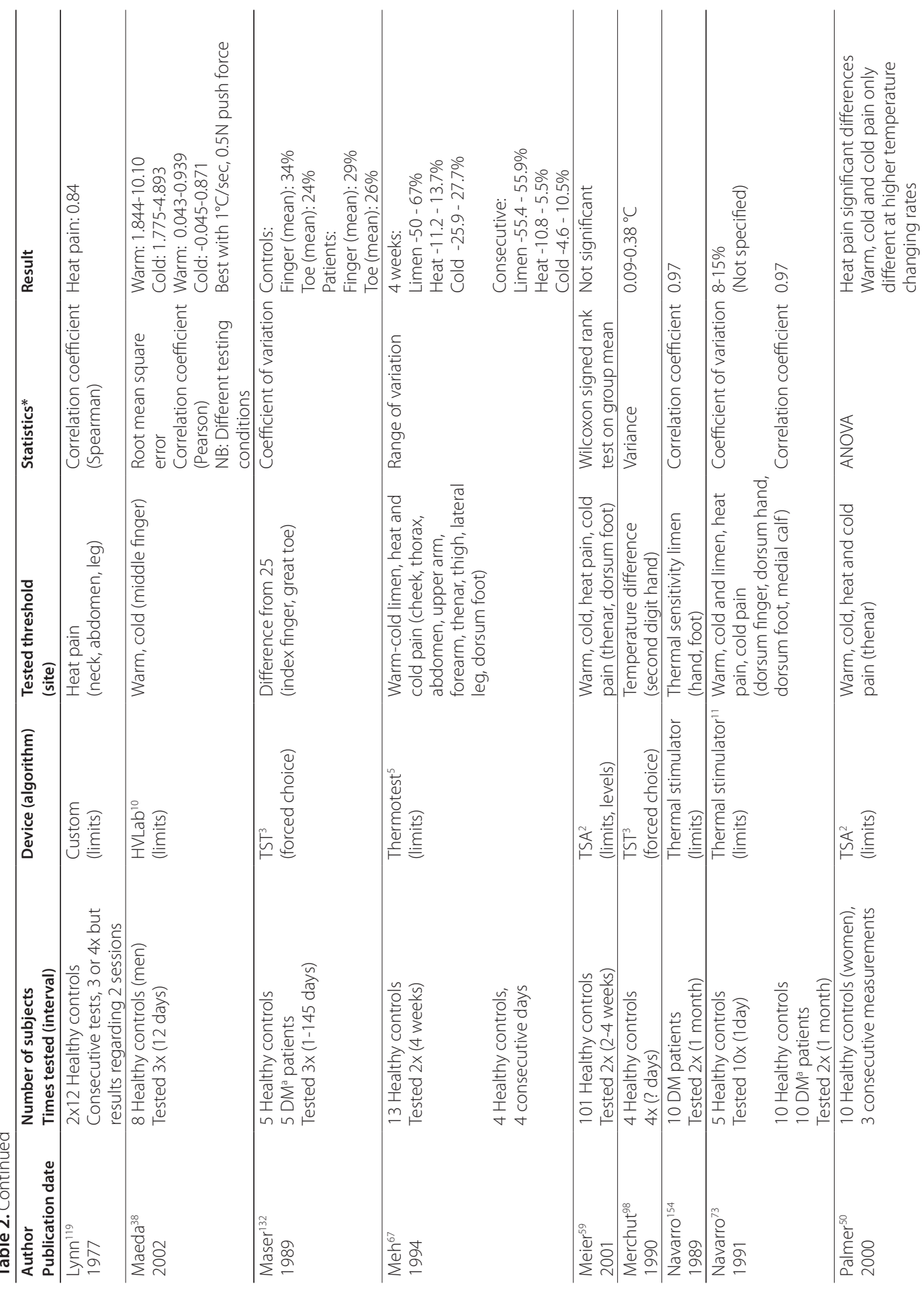




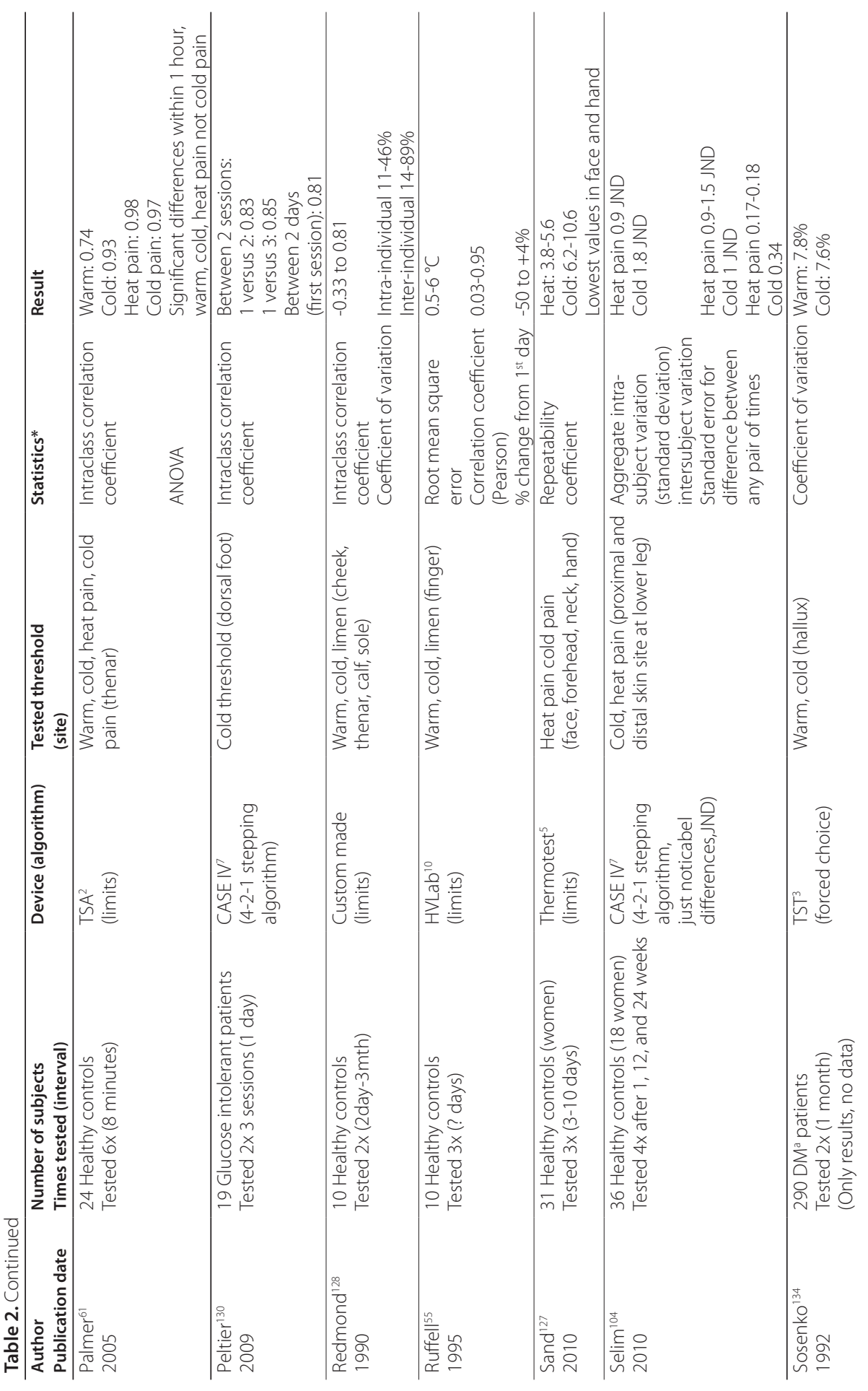




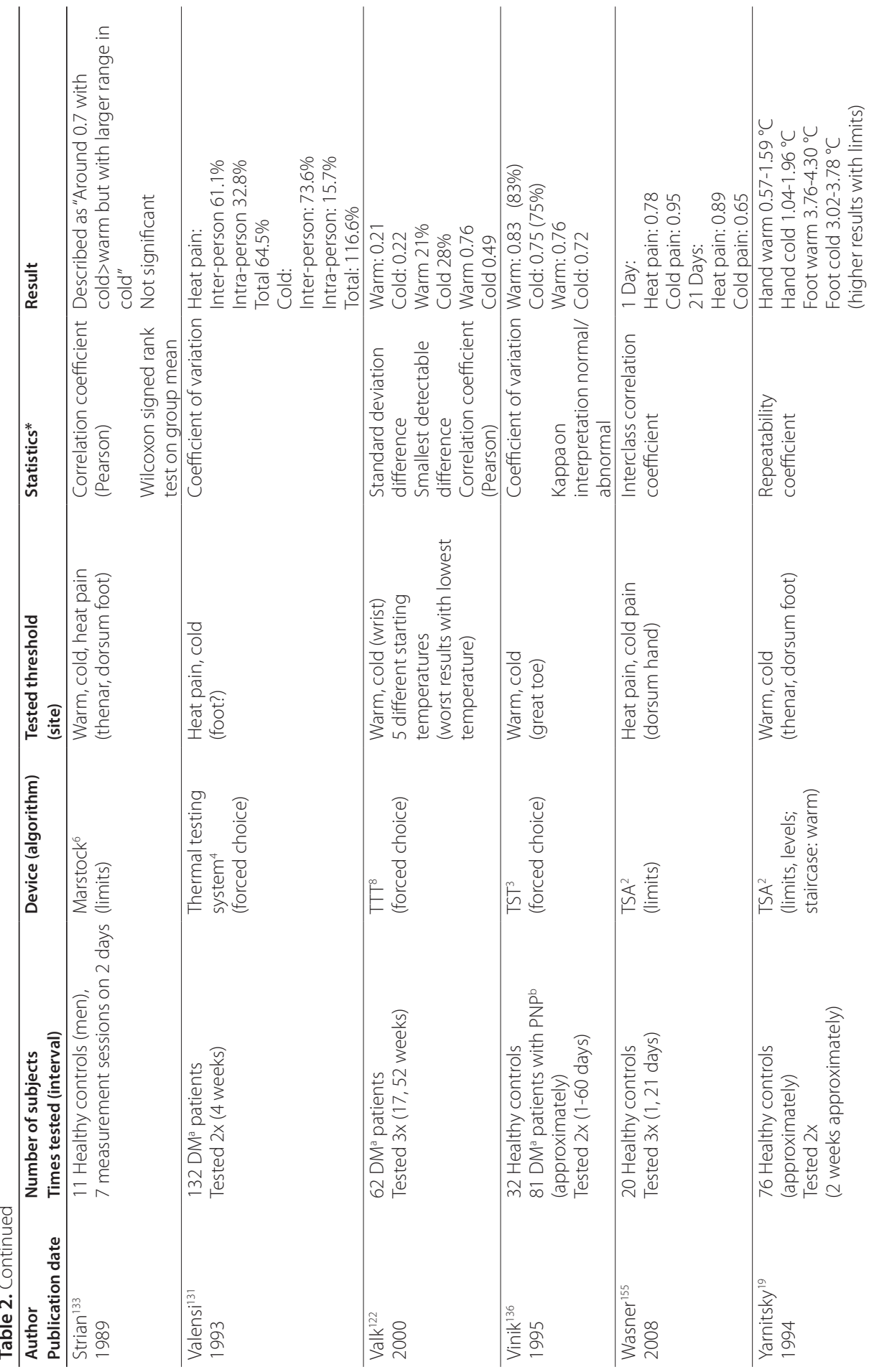




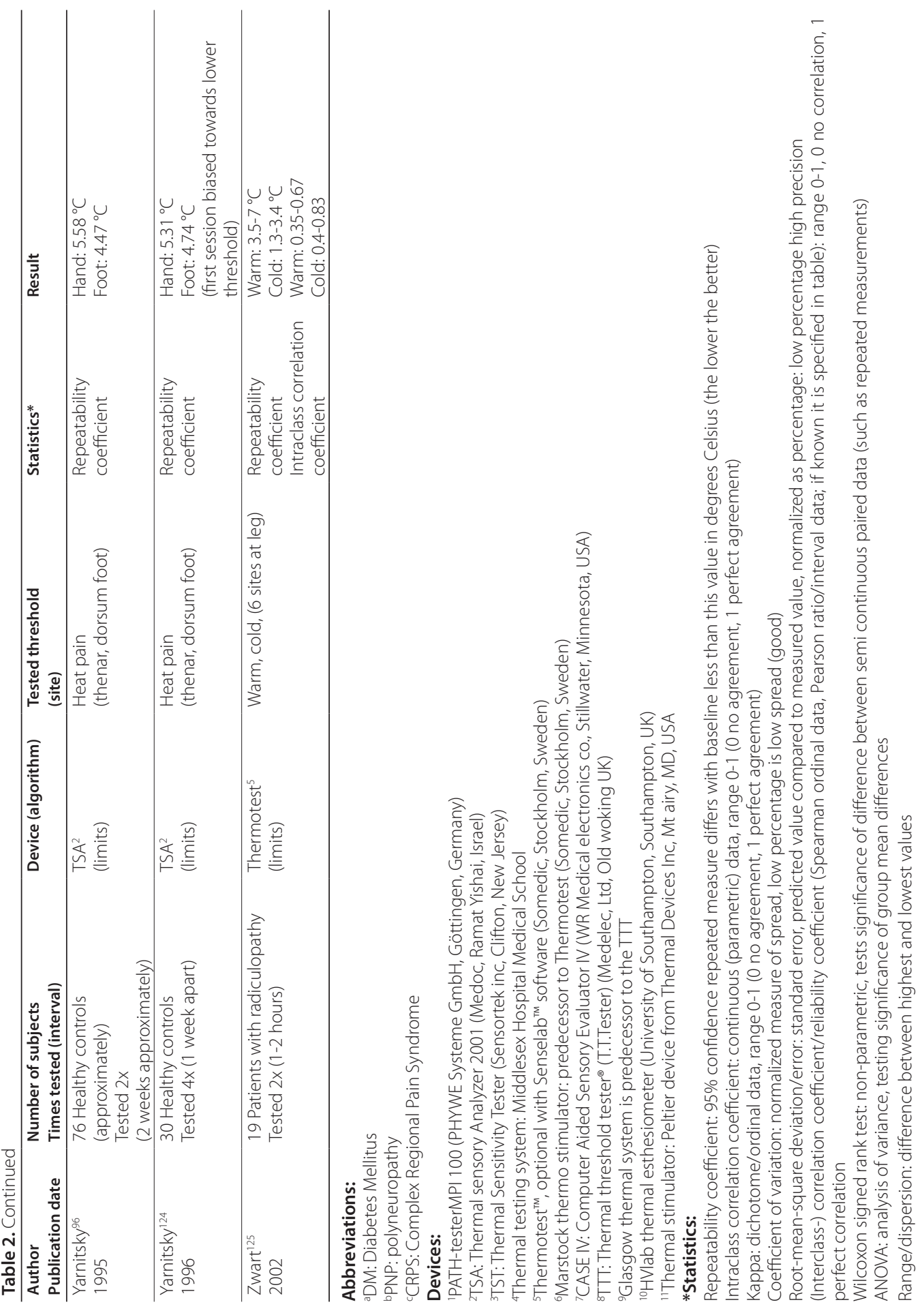

Chapter

3.1 


\section{REFERENCES}

1. Dyck PJ, Hughes RAC, O'Brien PC. Quantitating overall neuropathic symptoms, and outcomes. In: Dyck PJ, Thomas PK, eds. Peripheral neuropathy, 4th edition ed. Philadelphia: WB Saunders Company, 2005: 1031-1051.

2. Hoitsma E, Reulen JP, de Baets M, Drent M, Spaans F, Faber CG. Small fiber neuropathy: a common and important clinical disorder. J Neurol Sci 2004;227:119-130.

3. Fagius J, Wahren LK. Variability of sensory threshold determination in clinical use. J Neurol Sci 1981;51:1127.

4. Le Quesne PM, Fowler CJ, Parkhouse N. Peripheral neuropathy profile in various groups of diabetics. J Neurol Neurosurg Psychiatry 1990;53:558-563.

5. Armstrong FM, Bradburry JE, Ellis SH, et al. A study of peripheral diabetic neuropathy. The aplication of age related reference values. . Diabet Med 1991;8 S94-S99.

6. Bravenboer B, van Dam PS, Hop J, vd Steenhoven J, Erkelens DW. Thermal threshold testing for the assessment of small fibre dysfunction: normal values and reproducibility. Diabet Med 1992;9:546-549.

7. Claus D, Mustafa C, Vogel W, Herz M, Neundorfer B. Assessment of diabetic neuropathy: definition of norm and discrimination of abnormal nerve function. Muscle Nerve 1993;16:757-768.

8. Bartlett G, Stewart JD, Tamblyn R, Abrahamowicz M. Normal distributions of thermal and vibration sensory thresholds. Muscle Nerve 1998;21:367-374.

9. Kemler MA, Schouten HJ, Gracely RH. Diagnosing sensory abnormalities with either normal values or values from contralateral skin: comparison of two approaches in complex regional pain syndrome I. Anesthesiology 2000;93:718-727.

10. Blankenburg $M$, Boekens $H$, Hechler $T$, et al. Reference values for quantitative sensory testing in children and adolescents: developmental and gender differences of somatosensory perception. Pain 2010;149:76-88

11. Seah SA, Griffin MJ. Normal values for thermotactile and vibrotactile thresholds in males and females. Int Arch Occup Environ Health 2008;81:535-543.

12. Guy RJ, Clark CA, Malcolm PN, Watkins PJ. Evaluation of thermal and vibration sensation in diabetic neuropathy. Diabetologia 1985;28:131-137.

13. Hilz MJ, Stemper B, Axelrod FB, Kolodny EH, Neundorfer B. Quantitative thermal perception testing in adults. J Clin Neurophysiol 1999;16:462-471.

14. Fruhstorfer $\mathrm{H}$, Lindblom U, Schmidt WC. Method for quantitative estimation of thermal thresholds in patients. J Neurol Neurosurg Psychiatry 1976;39:1071-1075.

15. Lin YH, Hsieh SC, Chao CC, Chang YC, Hsieh ST. Influence of aging on thermal and vibratory thresholds of quantitative sensory testing. J Peripher Nerv Syst 2005;10:269-281.

16. Zinman LH, Bril V, Perkins BA. Cooling detection thresholds in the assessment of diabetic sensory polyneuropathy: comparison of CASE IV and Medoc instruments. Diabetes Care 2004;27:1674-1679.

17. Jamal GA, Hansen S, Weir Al, Ballantyne JP. An improved automated method for the measurement of thermal thresholds. 1. Normal subjects. J Neurol Neurosurg Psychiatry 1985;48:354-360.

18. Dyck PJ, O'Brien PC, Kosanke JL, Gillen DA, Karnes JL. A 4, 2, and 1 stepping algorithm for quick and accurate estimation of cutaneous sensation threshold. Neurology 1993;43:1508-1512.

19. Yarnitsky D, Sprecher E. Thermal testing: normative data and repeatability for various test algorithms. J Neurol Sci 1994;125:39-45.

20. Dyck PJ, Zimmerman I, Gillen DA, Johnson D, Karnes JL, O'Brien PC. Cool, warm, and heat-pain detection thresholds: testing methods and inferences about anatomic distribution of receptors. Neurology 1993;43:1500-1508.

21. Reulen JP, Lansbergen MD, Verstraete E, Spaans F. Comparison of thermal threshold tests to assess small nerve fiber function: limits vs. levels. Clin Neurophysiol 2003;114:556-563.

22. Dyck PJ, O'Brien PC, Johnson DM, Klein CJ, Dyck PJB. Quantitative sensation testing. In: Dyck PJ, Thomas PK, eds. Peripheral neuropathy, 4th edition ed. Philadelphia: WB Saunders Company, 2005: 1063-1093.

23. Defrin R, Shachal-Shiffer M, Hadgadg M, Peretz C. Quantitative somatosensory testing of warm and heat-pain thresholds: the effect of body region and testing method. Clin J Pain 2006;22:130-136.

24. Levy D, Abraham R, Reid G. A comparison of two methods for measuring thermal thresholds in diabetic neuropathy. J Neurol Neurosurg Psychiatry 1989;52:1072-1077. 
25. Claus D, Hilz MJ, Neundorfer B. Thermal discrimination thresholds: a comparison of different methods. Acta Neurol Scand 1990;81:533-540.

26. Kemler MA, Reulen JP, van Kleef M, Barendse GA, van den Wildenberg FA, Spaans F. Thermal thresholds in complex regional pain syndrome type I: sensitivity and repeatability of the methods of limits and levels. Clin Neurophysiol 2000;111:1561-1568.

27. Kojo I, Pertovaara A. The effects of stimulus area and adaptation temperature on warm and heat pain thresholds in man. Int J Neurosci 1987;32:875-880.

28. Defrin R, Urca G. Spatial summation of heat pain: a reassessment. Pain 1996;66:23-29.

29. Hilz MJ, Glorius SE, Schweibold G, Neuner I, Stemper B, Axelrod FB. Quantitative thermal perception testing in preschool children. Muscle Nerve 1996;19:381-383.

30. Hilz MJ, Stemper B, Schweibold G, Neuner I, Grahmann F, Kolodny EH. Quantitative thermal perception testing in 225 children and juveniles. J Clin Neurophysiol 1998;15:529-534.

31. Lautenbacher S, Nielsen J, Andersen T, Arendt-Nielsen L. Spatial summation of heat pain in males and females. Somatosens Mot Res 2001;18:101-105.

32. Defrin R, Ohry A, Blumen N, Urca G. Sensory determinants of thermal pain. Brain 2002;125:501-510.

33. Defrin R, Givon R, Raz N, Urca G. Spatial summation and spatial discrimination of pain sensation. Pain 2006;126:123-131.

34. Defrin R, Petrini L, Arendt-Nielsen L. Spatial summation of thermal sensations depends on skin type and skin sensitivity. Exp Brain Res 2009;198:29-36.

35. Green BG, Cruz A. "Warmth-insensitive fields": evidence of sparse and irregular innervation of human skin by the warmth sense. Somatosens Mot Res 1998;15:269-275.

36. Green BG, Zaharchuk R. Spatial variation in sensitivity as a factor in measurements of spatial summation of warmth and cold. Somatosens Mot Res 2001;18:181-190.

37. Pavlakovic G, Klinke I, Pavlakovic H, et al. Effect of thermode application pressure on thermal threshold detection. Muscle Nerve 2008;38:1498-1505.

38. Maeda S, Sakakibara H. Thermotactile perception thresholds measurement conditions. Ind Health 2002;40:353-361.

39. Pertovaara A, Kauppila T, Hamalainen MM. Influence of skin temperature on heat pain threshold in humans. Exp Brain Res 1996;107:497-503.

40. Nielsen J, Arendt-Nielsen L. The influence of rate of temperature change and peak stimulus duration on pain intensity and quality. Somatosens Mot Res 1998;15:220-229.

41. Helme RD, Meliala A, Gibson SJ. Methodologic factors which contribute to variations in experimental pain threshold reported for older people. Neurosci Lett 2004;361:144-146.

42. Schestatsky P, Algaba R, Perez D, et al. Transient decrease of sensory perception after thermoalgesic stimuli for quantitative sensory testing. Muscle Nerve 2007:36:466-470.

43. Agostinho $\mathrm{CM}$, Scherens $\mathrm{A}$, Richter $\mathrm{H}$, et al. Habituation and short-term repeatability of thermal testing in healthy human subjects and patients with chronic non-neuropathic pain. Eur J Pain 2009;13:779-785.

44. Croze S, Duclaux R. Thermal pain in humans: influence of the rate of stimulation. Brain Res 1978;157:418421.

45. Pertovaara A, Kojo I. Influence of the rate of temperature change on thermal thresholds in man. Exp Neurol 1985:87:439-445.

46. Swerup C, Nilsson BY. Dependence of thermal thresholds in man on the rate of temperature change. Acta Physiol Scand 1987;131:623-624.

47. Yarnitsky D, Ochoa JL. Studies of heat pain sensation in man: perception thresholds, rate of stimulus rise and reaction time. Pain 1990;40:85-91.

48. Yarnitsky D, Ochoa JL. Warm and cold specific somatosensory systems. Psychophysical thresholds, reaction times and peripheral conduction velocities. Brain 1991;114 (Pt 4):1819-1826.

49. Hilz MJ, Zimmermann P, Claus D, Neundorfer B. Thermal threshold determination in alcoholic polyneuropathy: an improvement of diagnosis. Acta Neurol Scand 1995;91:389-393.

50. Palmer ST, Martin DJ, Steedman WM, Ravey J. C-and Adelta-fibre mediated thermal perception: response to rate of temperature change using method of limits. Somatosens Mot Res 2000;17:325-333.

51. Hilz MJ, Glorius S, Beric A. Thermal perception thresholds: influence of determination paradigm and reference temperature. J Neurol Sci 1995;129:135-140. 
52. Kenshalo DR, Holmes CE. Warm and cool thresholds as a function of rate of stimulus temperature change. Perception and psychophysics 1968;3:81.

53. Harding LM, Loescher AR. Adaptation to warming but not cooling at slow rates of stimulus change in thermal threshold measurements. Somatosens Mot Res 2005;22:45-48.

54. Kenshalo DR, Nafe JP, Brooks B. Variations in Thermal Sensitivity. Science 1961;134:104-105.

55. Ruffell CM, Griffin MJ. Effect of starting temperature on the repeatability of thermotactile thresholds. Cent Eur J Public Health 1995;3 Suppl:81-84.

56. Hirosawa I, Dodo H, Hosokawa M, Watanabe S, Nishiyama K, Fukuchi Y. Physiological variations of warm and cool sense with shift of environmental temperature. Int J Neurosci 1984;24:281-288.

57. Pickering $G$, Jourdan D, Eschalier A, Dubray C. Impact of age, gender and cognitive functioning on pain perception. Gerontology 2002;48:112-118.

58. Kenshalo DR, Bergen DC. A device to measure cutaneous temperature sensitivity in humans and subhuman species. J Appl Physiol 1975;39:1038-1040.

59. Meier PM, Berde CB, DiCanzio J, Zurakowski D, Sethna NF. Quantitative assessment of cutaneous thermal and vibration sensation and thermal pain detection thresholds in healthy children and adolescents. Muscle Nerve 2001;24:1339-1345.

60. Kelly KG, Cook T, Backonja MM. Pain ratings at the thresholds are necessary for interpretation of quantitative sensory testing. Muscle Nerve 2005;32:179-184.

61. Palmer ST, Martin DJ. Thermal perception thresholds recorded using method of limits change over brief time intervals. Somatosens Mot Res 2005;22:327-334.

62. Croze S, Duclaux R, Russek M. Constancy of heat pain characteristics to changes in skin and body temperature. Brain Res 1977;131:367-372.

63. Hagander LG, Midani HA, Kuskowski MA, Parry GJ. Quantitative sensory testing: effect of site and pressure on vibration thresholds. Clin Neurophysiol 2000;111:1066-1069.

64. Fowler CJ, Carroll MB, Burns D, Howe N, Robinson K. A portable system for measuring cutaneous thresholds for warming and cooling. J Neurol Neurosurg Psychiatry 1987;50:1211-1215.

65. Halar EM, Hammond MC, LaCava EC, Camann C, Ward J. Sensory perception threshold measurement: an evaluation of semiobjective testing devices. Arch Phys Med Rehabil 1987;68:499-507.

66. Sosenko JM, Kato M, Soto R, Ayyar DR. Determinants of quantitative sensory testing in non-neuropathic individuals. Electromyogr Clin Neurophysiol 1989;29:459-463.

67. Meh D, Denislic M. Quantitative assessment of thermal and pain sensitivity. J Neurol Sci 1994;127:164169.

68. Meh D, Denislic M. Influenc of sympathetic activity, temperature, ischemia and diazepam on thermal and vibration thresholds. Pflugers Arch 1996;431:R305-306.

69. Bertelsmann FW, Heimans JJ, Weber EJ, van der Veen EA, Schouten JA. Thermal discrimination thresholds in normal subjects and in patients with diabetic neuropathy. J Neurol Neurosurg Psychiatry 1985;48:686690.

70. Arezzo JC, Schaumburg HH, Laudadio C. Thermal sensitivity tester. Device for quantitative assessment of thermal sense in diabetic neuropathy. Diabetes 1986;35:590-592.

71. Hansen S, Jamal GA, Weir Al, Ballantyne JP, Bissessar EA. An instrument for measurement of thermal thresholds in man. Biosensors 1987;3:391-401.

72. Ziegler D, Mayer P, Wiefels K, Gries FA. Assessment of small and large fiber function in long-term type 1 (insulin-dependent) diabetic patients with and without painful neuropathy. Pain 1988;34:1-10.

73. Navarro X, Kennedy WR. Evaluation of thermal and pain sensitivity in type I diabetic patients. J Neurol Neurosurg Psychiatry 1991;54:60-64.

74. Verdugo R, Ochoa JL. Quantitative somatosensory thermotest. A key method for functional evaluation of small calibre afferent channels. Brain 1992;115 ( Pt 3):893-913.

75. Greenspan JD, Taylor DJ, McGillis SL. Body site variation of cool perception thresholds, with observations on paradoxical heat. Somatosens Mot Res 1993;10:467-474.

76. Redmond JM, McKenna MJ, Feingold M, Ahmad BK. Sensory testing is different in laboratory personnel compared with paid volunteers. Muscle Nerve 1995;18:351-352.

77. Stevens JC, Choo KK. Temperature sensitivity of the body surface over the life span. Somatosens Mot Res 1998;15:13-28.

78. Taylor DJ, McGillis SL, Greenspan JD. Body site variation of heat pain sensitivity. Somatosens Mot Res 1993;10:455-465. 
79. Gerr F, Letz R. Covariates of human peripheral nerve function: II. Vibrotactile and thermal thresholds. Neurotoxicol Teratol 1994;16:105-112.

80. Gelber DA, Pfeifer MA, Broadstone VL, et al. Components of variance for vibratory and thermal threshold testing in normal and diabetic subjects. J Diabetes Complications 1995;9:170-176.

81. Harrison JL, Davis KD. Cold-evoked pain varies with skin type and cooling rate: a psychophysical study in humans. Pain 1999;83:123-135.

82. Essick G, Guest S, Martinez E, Chen C, McGlone F. Site-dependent and subject-related variations in perioral thermal sensitivity. Somatosens Mot Res 2004;21:159-175.

83. Rolke R, Baron R, Maier C, et al. Quantitative sensory testing in the German Research Network on Neuropathic Pain (DFNS): standardized protocol and reference values. Pain 2006;123:231-243.

84. Green BG. Heat pain thresholds in the oral-facial region. Percept Psychophys 1985;38:110-114.

85. Claus D, Hilz MJ, Hummer I, Neundorfer B. Methods of measurement of thermal thresholds. Acta Neurol Scand 1987;76:288-296.

86. Long DA. Hand differences and reported intensity of nociceptive stimuli. Percept Mot Skills 1994;79:411417.

87. Becser N, Sand T, Zwart JA. Reliability of cephalic thermal thresholds in healthy subjects. Cephalalgia 1998;18:574-582.

88. Schaffner N, Wittwer A, Kut E, Folkers G, Benninger DH, Candia V. Heat pain threshold and tolerance show no left-right perceptual differences at complementary sites of the human forearm. Neurosci Lett 2008;440:309-313.

89. Pigg M, Baad-Hansen L, Svensson P, Drangsholt M, List T. Reliability of intraoral quantitative sensory testing (QST). Pain 2010;148:220-226.

90. Doeland HJ, Nauta JJ, van Zandbergen JB, et al. The relationship of cold and warmth cutaneous sensation to age and gender. Muscle Nerve 1989;12:712-715.

91. Dyck PJ, Litchy WJ, Lehman KA, Hokanson JL, Low PA, O'Brien PC. Variables influencing neuropathic endpoints: the Rochester Diabetic Neuropathy Study of Healthy Subjects. Neurology 1995;45:1115-1121.

92. Huang HW, Wang WC, Lin CC. Influence of age on thermal thresholds, thermal pain thresholds, and reaction time. J Clin Neurosci 2010;17:722-726.

93. Ijff GA, Bertelsmann FW, Nauta JJ, Heimans JJ. Cold and warm cutaneous sensation in diabetic patients. Diabet Med 1991;8 Spec No:S71-73.

94. Lautenbacher S, Kunz M, Strate P, Nielsen J, Arendt-Nielsen L. Age effects on pain thresholds, temporal summation and spatial summation of heat and pressure pain. Pain 2005;115:410-418.

95. Stevens JC, Cruz LA, Marks LE, Lakatos S. A multimodal assessment of sensory thresholds in aging. J Gerontol B Psychol Sci Soc Sci 1998;53:P263-272.

96. Yarnitsky D, Sprecher E, Zaslansky R, Hemli JA. Heat pain thresholds: normative data and repeatability. Pain 1995;60:329-332.

97. Levy DM, Abraham RR, Abraham RM. Small- and large-fiber involvement in early diabetic neuropathy: a study with the medial plantar response and sensory thresholds. Diabetes Care 1987;10:441-447.

98. Merchut MP, Toleikis SC. Aging and quantitative sensory thresholds. Electromyogr Clin Neurophysiol 1990;30:293-297.

99. Donaghue KC, Bonney M, Simpson JM, et al. Autonomic and peripheral nerve function in adolescents with and without diabetes. Diabet Med 1993;10:664-671.

100. Harju EL. Cold and warmth perception mapped for age, gender, and body area. Somatosens Mot Res 2002;19:61-75.

101. Golja P, Tripton MJ, Mekjavic IB. Cutaneous thermal thresholds - the reproducibility of their measurements and the effect of gender. Journal of thermal biology 2003;28:341-346.

102. Li X, Petrini L, Defrin R, Madeleine P, Arendt-Nielsen L. High resolution topographical mapping of warm and cold sensitivities. Clin Neurophysiol 2008;119:2641-2646.

103. Klatzkin RR, Mechlin B, Girdler SS. Menstrual cycle phase does not influence gender differences in experimental pain sensitivity. Eur J Pain 2010;14:77-82.

104. Selim MM, Wendelschafer-Crabb G, Hodges JS, et al. Variation in quantitative sensory testing and epidermal nerve fiber density in repeated measurements. Pain 2010;151:575-581.

105. Fillingim RB, MadduxV, Shackelford JA. Sex differences in heat pain thresholds as a function of assessment method and rate of rise. Somatosens Mot Res 1999;16:57-62. 
106. Torgen M, Swerup C. Individual factors and physical work load in relation to sensory thresholds in a middleaged general population sample. Eur J Appl Physiol 2002;86:418-427.

107. Lautenbacher S, Strian F. Sex differences in pain and thermal sensitivity: the role of body size. Percept Psychophys 1991;50:179-183.

108. Lautenbacher S, Strian F. The role of body size in somatosensory testing. Electromyogr Clin Neurophysiol 1993;33:113-118.

109. Edwards RR, Fillingim RB. Ethnic differences in thermal pain responses. Psychosom Med 1999;61:346-354.

110. Yosipovitch G, Meredith G, Chan YH, Goh CL. Do ethnicity and gender have an impact on pain thresholds in minor dermatologic procedures? A study on thermal pain perception thresholds in Asian ethinic groups. Skin Res Technol 2004;10:38-42.

111. Watson PJ, Latif RK, Rowbotham DJ. Ethnic differences in thermal pain responses: a comparison of South Asian and White British healthy males. Pain 2005;118:194-200.

112. Pavlakovic G, Zuchner K, Zapf A, et al. Influence of intrinsic noise generated by a thermotesting device on thermal sensory detection and thermal pain detection thresholds. Muscle Nerve 2009;40:257-263.113. Kundermann B, Spernal J, Huber MT, Krieg JC, Lautenbacher S. Sleep deprivation affects thermal pain thresholds but not somatosensory thresholds in healthy volunteers. Psychosom Med 2004;66:932-937.

114. Onen SH, Alloui A, Gross A, Eschallier A, Dubray C. The effects of total sleep deprivation, selective sleep interruption and sleep recovery on pain tolerance thresholds in healthy subjects. J Sleep Res 2001;10:3542.

115. Defrin R, Pick CG, Peretz C, Carmeli E. A quantitative somatosensory testing of pain threshold in individuals with mental retardation. Pain 2004;108:58-66.

116. Hobart JC, Lamping DL, Thompson AJ. Evaluating neurological outcome measures: the bare essentials. J Neurol Neurosurg Psychiatry 1996;60:127-130.

117. Liang MH. Evaluating measurement responsiveness. J Rheumatol 1995;22:1191-1192.

118. Bird SJ, Brown MJ, Spino C, Watling S, Foyt HL. Value of repeated measures of nerve conduction and quantitative sensory testing in a diabetic neuropathy trial. Muscle Nerve 2006;34:214-224.

119. Lynn B, Perl ER. A comparison of four tests for assessing the pain sensitivity of different subjects and test areas. Pain 1977;3:353-365

120. Abad F, Diaz-Gomez NM, Rodriguez I, Perez R, Delgado JA. Subclinical pain and thermal sensory dysfunction in children and adolescents with Type 1 diabetes mellitus. Diabet Med 2002;19:827-831.

121. Hendriksen PH, Oey PL, Wieneke GH, Bravenboer B, van Huffelen AC. Subclinical diabetic polyneuropathy: early detection of involvement of different nerve fibre types. J Neurol Neurosurg Psychiatry 1993;56:509514.

122. Valk GD, Grootenhuis PA, van Eijk JT, Bouter LM, Bertelsmann FW. Methods for assessing diabetic polyneuropathy: validity and reproducibility of the measurement of sensory symptom severity and nerve function tests. Diabetes Res Clin Pract 2000;47:87-95.

123. Bland JM, Altman DG. Statistical methods for assessing agreement between two methods of clinical measurement. Lancet 1986;1:307-310

124. Yarnitsky D, Sprecher E, Zaslansky R, Hemli JA. Multiple session experimental pain measurement. Pain 1996;67:327-333.

125. Zwart JA, Sand T. Repeatability of dermatomal warm and cold sensory thresholds in patients with sciatica. Eur Spine J 2002;11:441-446.

126. Heldestad V, Linder J, Sellersjo L, Nordh E. Reproducibility and influence of test modality order on thermal perception and thermal pain thresholds in quantitative sensory testing. Clin Neurophysiol 2010;121:18781885.

127. Sand T, Nilsen KB, Hagen K, Stovner LJ. Repeatability of cold pain and heat pain thresholds: The application of sensory testing in migraine research. Cephalalgia 2010;30:904-909.

128. Redmond J, Cros D, Shahani BT. Variability of quantitative sensory testing: implications for clinical practice. Henry Ford Hosp Med J 1990;38:62-67.

129. Dyck PJ, Kratz KM, Lehman KA, et al. The Rochester Diabetic Neuropathy Study: design, criteria for types of neuropathy, selection bias, and reproducibility of neuropathic tests. Neurology 1991;41:799-807.

130. Peltier A, Smith AG, Russell JW, et al. Reliability of quantitative sudomotor axon reflex testing and quantitative sensory testing in neuropathy of impaired glucose regulation. Muscle Nerve 2009;39:529-535. 
131. Valensi P, Attali JR, Gagant S. Reproducibility of parameters for assessment of diabetic neuropathy. The French Group for Research and Study of Diabetic Neuropathy. Diabet Med 1993;10:933-939.

132. Maser RE, Nielsen VK, Bass EB, et al. Measuring diabetic neuropathy. Assessment and comparison of clinical examination and quantitative sensory testing. Diabetes Care 1989;12:270-275.

133. Strian F, Lautenbacher S, Galfe G, Holzl R. Diurnal variations in pain perception and thermal sensitivity. Pain 1989;36:125-131.

134. Sosenko JM, Kato M, Soto R, Goldberg RB. Sensory function at diagnosis and in early stages of NIDDM in patients detected through screening. Diabetes Care 1992;15:847-852.

135. Benbow SJ, Chan AW, Bowsher D, MacFarlane IA, Williams G. A prospective study of painful symptoms, small-fibre function and peripheral vascular disease in chronic painful diabetic neuropathy. Diabet Med 1994;11:17-21

136. Vinik Al, Suwanwalaikorn S, Stansberry KB, Holland MT, McNitt PM, Colen LE. Quantitative measurement of cutaneous perception in diabetic neuropathy. Muscle Nerve 1995;18:574-584.

137. Lowenstein L, Jesse K, Kenton K. Comparison of perception threshold testing and thermal-vibratory testing. Muscle Nerve 2008;37:514-517.

138. Apfel SC, Kessler JA, Adornato BT, Litchy WJ, Sanders C, Rask CA. Recombinant human nerve growth factor in the treatment of diabetic polyneuropathy. NGF Study Group. Neurology 1998;51:695-702.

139. MCArthur JC, Yiannoutsos C, Simpson DM, et al. A phase II trial of nerve growth factor for sensory neuropathy associated with HIV infection. AIDS Clinical Trials Group Team 291. Neurology 2000;54:10801088.

140. Kramer HH, Rolke R, Hecht M, Bickel A, Birklein F. Follow-up of advanced diabetic neuropathy: useful variables and possible pitfalls. J Neurol 2005;252:315-320.

141. Schiffmann R, Hauer P, Freeman B, et al. Enzyme replacement therapy and intraepidermal innervation density in Fabry disease. Muscle Nerve 2006;34:53-56.

142. Sosenko JM, Kato M, Soto R, Bild DE. A prospective study of sensory function in patients with type 2 diabetes. Diabet Med 1993;10:110-114.

143. Brown MJ, Bird SJ, Watling S, et al. Natural progression of diabetic peripheral neuropathy in the Zenarestat study population. Diabetes Care 2004;27:1153-1159.

144. Schiffmann R, Floeter MK, Dambrosia JM, et al. Enzyme replacement therapy improves peripheral nerve and sweat function in Fabry disease. Muscle Nerve 2003;28:703-710.

145. Rolke R, Magerl W, Campbell KA, et al. Quantitative sensory testing: a comprehensive protocol for clinical trials. Eur J Pain 2006;10:77-88

146. Schepers RJ, Ringkamp M. Thermoreceptors and thermosensitive afferents. Neurosci Biobehav Rev 2009;33:205-212.

147. Shy ME, Frohman EM, So YT, et al. Quantitative sensory testing: report of the Therapeutics and Technology Assessment Subcommittee of the American Academy of Neurology. Neurology 2003;60:898-904.

148. Kenshalo DR, Sr. Somesthetic sensitivity in young and elderly humans. J Gerontol 1986;41:732-742.

149. de Neeling JN, Beks PJ, Bertelsmann FW, Heine RJ, Bouter LM. Sensory thresholds in older adults: reproducibility and reference values. Muscle Nerve 1994;17:454-461.

150. Leonhardt H, Meinecke D, Mustafa T, Gerlach KL. Newly-developed measuring device for the quantitative assessment of thermal and pain thresholds of peripheral nerves. Biomed Tech (Berl) 2005:50:268-270.

151. Dyck PJ, Zimmerman IR, Johnson DM, et al. A standard test of heat-pain responses using CASE IV. J Neurol Sci 1996;136:54-63.

152. Geber C, Klein T, Azad S, et al. Test-retest and interobserver reliability of quantitative sensory testing according to the protocol of the German Research Network on Neuropathic Pain (DFNS): a multi-centre study. Pain 2011;152:548-556.

153. Hilz MJ, Claus D, Neundorfer B. Early diagnosis of diabetic small fiber neuropathy by disturbed cold perception. J Diabet Complications 1988;2:38-43

154. Navarro X, Kennedy WR, Fries TJ. Small nerve fiber dysfunction in diabetic neuropathy. Muscle Nerve 1989;12:498-507.

155. Wasner GL, Brock JA. Determinants of thermal pain thresholds in normal subjects. Clin Neurophysiol 2008;119:2389-2395. 

Chapter 3.2

\section{OPTIMIZING TEMPERATURE THRESHOLD TESTING IN}

SMALL FIBER NEUROPATHY

Mayienne Bakkers, MD', Catharina G. Faber, MD, PhD', Jos P.H. Reulen, PhD'2, Janneke G.J. Hoeijmakers, MD', Els K. Vanhoutte, MD', Ingemar S.J. Merkies, MD, PhD ${ }^{3}$

'Department of Neurology, Maastricht University Medical Center, Maastricht, the Netherlands;

${ }^{2}$ Department of Clinical Neurophysiology, Maastricht University Medical Center, Maastricht, the Netherlands;

${ }^{3}$ Department of Neurology, Spaarne Hospital, Hoofddorp, the Netherlands. 


\section{ABSTRACT}

Introduction: This study examines optimization of a temperature threshold testing (TTT) protocol for patients with suspected small fiber neuropathy (SFN) to lessen the burden for both patients and technicians, without sacrificing accuracy.

Methods: Data from 81 patients with SFN (skin biopsy and TTT abnormal) and 81 without SFN (skin biopsy and TTT normal) were used. Warm, cold, and heat pain sensation thresholds were determined bilaterally on the thenar eminence and foot dorsum by methods of limits and levels. Diagnostic accuracy was determined for various sensory modality combinations through comparative corresponding area under the receiver-operator-characteristic curves.

Results: Assessment of warm and cold thresholds in all extremities by method of levels showed the best discriminatory ability (area under the curve: 0.95, sensitivity 84.2\%, specificity 93.8\%).

Discussion: These assessments are suggested for TTT examination in possible SFN patients. By applying this combination, the time needed for TTT can be reduced, maintaining diagnostic accuracy. 


\section{INTRODUCTION}

No gold standard exists for diagnosing small fiber neuropathy (SFN), though combinations of clinical symptoms and signs, intra-epidermal nerve fiber density (IENFD) reduction, and temperature threshold testing (TTT) abnormalities have been proposed..$^{1-3}$ Although recommendations for the use of TTT have been published ${ }_{1}^{4}$ no consensus exists regarding the preferred standardized TTT method to be used in suspected SFN. 5,6 The German Research Network on Neuropathic Pain (DFNS) has proposed a reaction time-dependent method, testing both cold and warm detection and heat and cold pain thresholds of the affected area, 7,8 whereas other groups test cold detection and heat pain thresholds of the foot with a reaction time-independent protocol. ${ }^{9}$ In some studies it is unclear whether examinations are performed unilaterally or bilaterally. ${ }^{10,11}$ A recent consensus meeting provided recommended indications for quantitative sensory testing in clinical practice and methods to perform and interpret quantitative sensory tests. ${ }^{12}$ The usefulness of TTT in SFN is described, emphasizing the need for a standardized protocol, adequate equipment, trained staff, and normative values.

In our Neurophysiology department, a standardized, extensive TTT protocol, assessing warm and cold sensation in both hands and feet through both the methods of levels and limits, and heat pain with the method of limits, has been applied since 1998.13,14 Stringent criteria for abnormality have been implemented based on published normative values. ${ }^{15,16} 17$ However, this protocol is time consuming, often exceeding 1.5 hours, which is considered a burden by patients and technicians.

This study aims to define the optimal TTT protocol that may reduce the burden for patients, without loss of diagnostic accuracy by comparing various combined sensory modalities of TTT systematically.

\section{METHODS}

\section{Patients}

Patients referred to the Maastricht University Medical Center (MUMC) for evaluation of possible SFN were asked to participate in this study. Data were collected between January 2006 and January 2011. Inclusion criteria were: age $\geq 18$ years; symptoms compatible with SFN, not otherwise explained; ${ }^{1,3}$ no signs of large nerve fiber involvement on neurological examination or nerve conduction studies; cooperation with temperature threshold testing and skin biopsy procedures (see below); on stable doses of medications for neuropathic pain and not taking any sedatives. Patients were asked to locate their (burning) pain on a human cartoon picture. 


\section{Temperature threshold testing}

Temperature threshold testing (TTT) was performed with a Temperature Sensory Analyzer (TSA II, Medoc', Ramat Yishai, Israel). Warm, cool, and heat pain detection thresholds were tested. Patients were seated comfortably in a temperature-controlled room $\left(\sim 23^{\circ} \mathrm{C}\right)$. Instructions were provided to the patients in a standardized manner prior to the examination. The thermode $(30 \times 30 \mathrm{~mm})$ was applied with light pressure with Velcro straps to the testing sites. Testing sites were both left and right thenar eminence and dorsal foot. The testing order consisted of right thenar, right foot, left thenar, and left foot. Baseline thermode temperature was $32^{\circ} \mathrm{C}$, rise time $1{ }^{\circ} \mathrm{C} /$ second, and the temperature range was $0^{\circ}$ to $50^{\circ} \mathrm{C}$. Per site, TTT was performed according to the method of limits (reaction time dependent: pushing a button when change in temperature or pain is sensed) and to the method of levels (reaction time independent: per stimulus answering whether a warmer or cooler temperature is sensed). Heat pain threshold was then performed with the method of limits. ${ }^{14-16}$ Per site, the testing order was warm-limits and cold-limits (mean of 5 ramped stimuli, 2 test stimuli), warm-levels (a predefined protocol with random null stimuli), cold-levels, heat pain (mean of 4, 1 test stimulus). For the method of levels, patients were instructed only to consider the tested sensation, and were asked to not give a positive response when they did not feel warming of the element, but only felt the cooling (return to baseline) when warm threshold was tested. The thresholds were compared to published normative values, using age-corrected Z-values > 2.5 as cut-off values. ${ }^{15-17}$ In the original testing protocol, the final conclusion that the warm or cold temperature detection threshold of a tested site was abnormal was only made if the results of both the methods of limits and levels were abnormal for that site $\left(\mathrm{TTT}_{\text {Lev+Lim+ }}\right)$. If in at least 1 extremity the detection thresholds, determined by the methods of limits and levels was abnormal, the TTT result was considered abnormal for that patient.

\section{Skin biopsy}

In all participants, intra-epidermal nerve fiber density (IENFD) was determined through skin biopsy examination according to international guidelines. ${ }^{18}$ IENFD was abnormal if the value was below the $5^{\text {th }}$ percentile value according to age- and gender-matched normative values. ${ }^{19}$

\section{Study design}

The study was approved by the medical ethics committee of the MUMC (Central Committee for Human Related Research, identifier number p06.0066L/MEC 05224) in accordance with the guidelines of the declaration of Helsinki (amendment October 2008, Seoul, Korea). Informed consent was obtained from all patients before examinations. All patients were examined at the MUMC. 


\section{Predefined combinations of sensory modalities in TTT to be examined}

Figure 1 shows the analysis flowchart based on consensus of 2 neuromuscular physicians (ISJM, CGF). A practical approach was chosen, aiming to keep the combinations as logical as possible for clinical application. Warm and cold threshold tests were incorporated, as they are less burdensome to patients than pain testing. Testing either warm or cold compared to both was important, as they test different subsets of fibers. ${ }^{20}$ A two-sided approach was chosen (testing both right and left extremities), as symptoms may be asymmetric. ${ }^{21,22}$ Hence, a total of 26 combinations (excluding the combination of the original testing protocol, see last row of boxes) were predefined for comparison purposes. After using this flowchart for analysis, the one-sided (right side and left side examined separately) findings of all combinations (adding another $2 \times 26$ assessments) were also examined to determine whether further reduction could be achieved (total number of combined assessments $n=78$ ).

Figure 1. Systematic approach to temperature threshold testing.

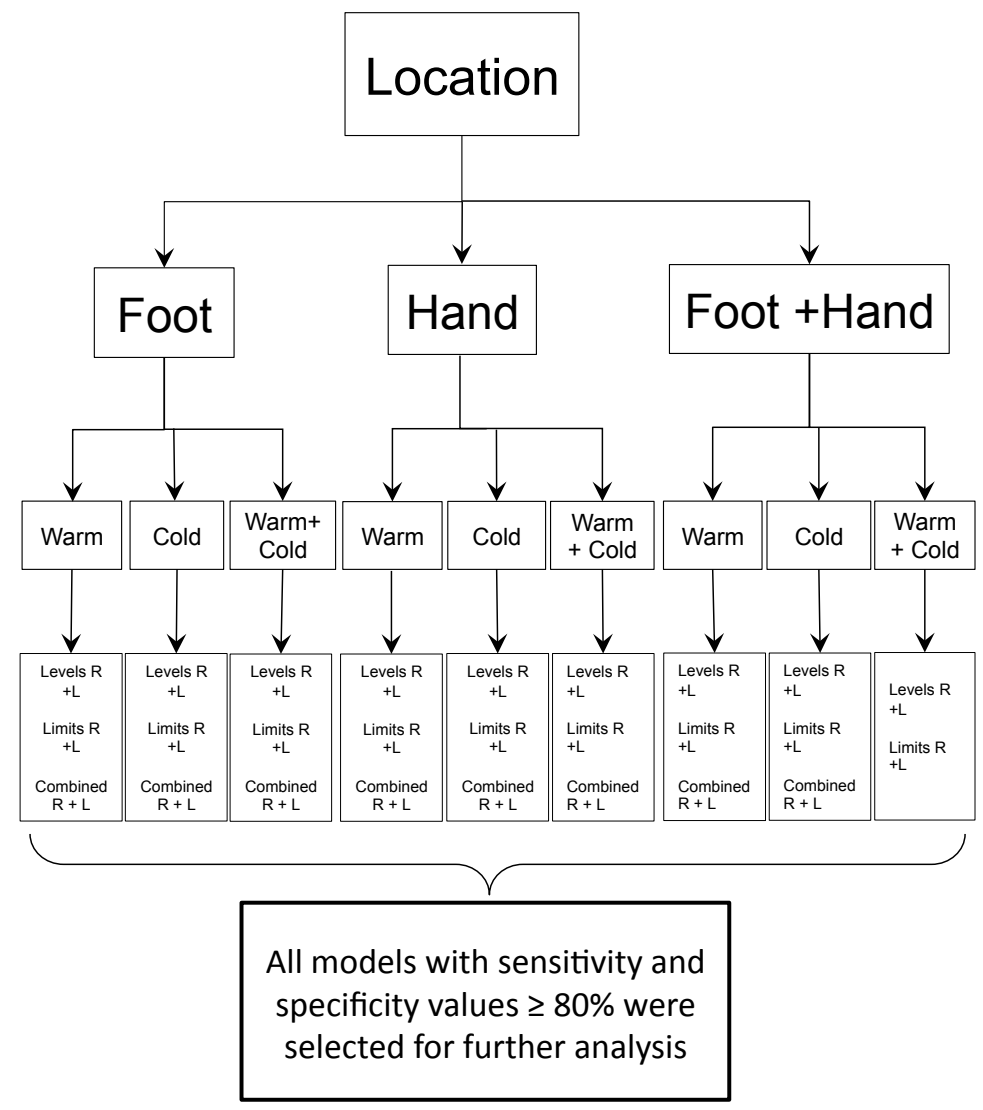

$\mathrm{R}=$ right side of body; $\mathrm{L}=$ left side of body; Combined $=$ method of levels + method of limits combined. 


\section{Statistical analysis}

General aspects

Sensory TTT modalities were expressed as (ab)normal for each test separately (e.g. warm threshold according to the method of levels at the thenar eminence, warm threshold according to the method of limits at the thenar eminence, cold threshold according to method of levels at the thenar eminence, etc.), using age- and genderspecific normative values and age-corrected Z-values $>2.5$ as cut-off value..$^{15,16}$

Patient data

Based on the results of the different assessments, eligible patients were divided into 3 groups. Group 1, patients with a definite diagnosis of SFN according to strict criteria: $\geq 2$ symptoms compatible with SFN and not otherwise explained, reduced IENFD at the distal leg, and abnormal TTT findings of at least 1 extremity (SFN ${ }_{\text {def }}$ group). Group 2, patients who met the strict criteria of having normal neurological examination and nerve conduction studies, normal IENFD, and normal TTT findings (SFN ${ }_{\text {neg }}$ group). Group 3, patients with symptoms compatible with SFN and either reduced IENFD or

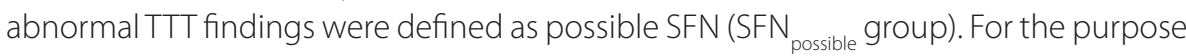
of optimizing the TTT protocol, the data of patients with a definite diagnosis $\left(\mathrm{SFN}_{\text {def }}\right.$ and $\mathrm{SFN}_{\text {neg }}$ groups) were used.

The basic characteristics (gender, age, and duration of symptoms) were compared

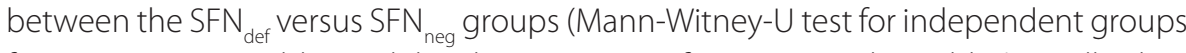
for continuous variables and the chi-squares test for categorical variables). Finally, data of all patients were used to determine the shift in diagnosis classification comparison results of the new TTT protocol to those of the original TTT protocol (TTT LevtLim+ $_{+}$).

\section{Logistic regression studies}

The SFN $_{\text {def }}$ group was selected as the "validation gold standard" cohort and was compared with the SFN ${ }_{\text {neg }}$ group. Through univariate logistic regressions, areas-underthe-receiver-operator-characteristic-curves (AUC-ROC) were computed. The diagnostic accuracy (AUC-ROC findings) of all postulated combinations of sensory modalities (figure 1) was compared to the original testing protocol (TTT LevtLim+ $_{+}$). Sensitivity (fraction of SFN positive cases classified correctly) and specificity (fraction of negative cases classified correctly) scores as well as percentage of subjects classified correctly as having SFN or not were calculated. AUC values were defined as follows: $\leq 0.5$, does not discriminate; values between 0.7-0.8, acceptable discrimination; values between 0.8-0.9, excellent discrimination; and values $\geq 0.9$, outstanding discrimination. ${ }^{23}$ To determine which sensory combination differentiates best between SFN def $_{\text {and }}$ SFN ${ }_{\text {neg' }}$ all combinations exceeding an arbitrarily chosen $80 \%$ cut-off score for sensitivity and specificity were selected subsequently for final comparison. The AUC values for these selected sensory combinations were compared subsequently using the Stata roccomp command, which tests the equality of 2 or more ROC areas obtained from the same sample of patients. ${ }^{24}$

All analyses were performed using Stata 12.0 for Windows XP (Stata Statistical Software: 
Release 12.0. Stata Corporation; 2010). P-values $<0.05$ were considered statistically significant.

\section{RESULTS}

\section{Patient characteristics}

A total of 317 patients were evaluated initially. Eighty-one met the strict criteria for SFN $\left(S_{\text {def }}\right)$, and 81 did not have SFN $\left(\right.$ SFN $\left._{\text {neg }}\right)$. The basic characteristics are listed in Table 1. SFN was considered to be idiopathic in 33 patients and due to sarcoidosis in 27. Etiologies in other patients included SCN9A mutation (11 patients), diabetes (4), vitamin B6 toxicity (3), alcohol (1), Sjögren syndrome (1), and B. burgdorferi infection (1). Other than more men in the SFN ${ }_{\text {def }}$ group, there were no significant differences between the groups. Most patients had a symmetrical length-dependent clinical distribution of neuropathic symptoms, usually pain (Table 2).

Table 1. Basic characteristics of selected patients with strict SFN diagnosis $\left(S_{\text {fFN }}\right.$ def $)$ and patients with no SFN $\left(\mathrm{SFN}_{\text {neg }}\right)$.

\begin{tabular}{lccc}
\hline & $\begin{array}{c}\text { SFN } \\
\mathbf{n = 8 1}\end{array}$ & $\begin{array}{c}\mathbf{S F N}_{\text {neg }} \\
(\mathbf{n}=\mathbf{8 1})\end{array}$ & P-value \\
\hline Gender & & & \\
$\quad$ Women (\%) & $36(44.4)$ & $49(60.5)$ & 0.04 \\
$\quad$ Men (\%) & $45(55.6)$ & $32(39.5)$ & 0.31 \\
\hline Mean age in yrs (SD*), range & $50.0(12.7), 19-78$ & $51.9(12), 17-81$ & \\
\hline Duration of neuropathic symptoms, & & & 0.49 \\
yrs (SD*), range & $8.1(9.5), 0.4-50$ & $8.6(9), 0.5-50$ & \\
\hline
\end{tabular}

*SD=standard deviation

Table 2. Distribution of symptoms and signs in patients diagnosed with definite SFN ( $n=81)$

\begin{tabular}{ccc} 
Site & \multicolumn{2}{c}{ Location burning pain (\% of patients) } \\
& Left & Right \\
\hline Forearm & 22.2 & 20.4 \\
Hands & 40.7 & 40.7 \\
Fingers & 51.8 & 51.8 \\
Upper legs & 35.2 & 37 \\
Lower legs & 63 & 63 \\
Feet & 79.6 & 77.8 \\
Toes & 70.4 & 68.5 \\
\hline
\end{tabular}

\section{General findings, Diagnostic accuracy and Comparison studies}

A total of $77.9 \%$ of the patients had an abnormal TTT score in the feet versus $56.5 \%$ in the hands. TTT was abnormal in the feet and normal in the hands in $29.3 \%$ of patients (93/317). Conversely, the combination of abnormal TTT in the hands with normal TTT examination in the feet was seen in 25 patients (7.9\%). Abnormalities [irrespective of 
method (levels or limits) applied or quality (warm or cold) examined] were found in both feet and hands in $79 \%$ and $46.9 \%$, respectively in the SFN def subgroup.

The SFN def group was used to calculate the areas under the ROC curves with corresponding sensitivity, specificity, and percentage of patients correctly classified as having or not having SFN. The results of the diagnostic performances for the predefined combinations are listed in Table 3. Only 2 sets of combinations of TTT modalities using the method of levels $\left(\mathrm{TTT}_{\text {alternative } \mathrm{A}}\right.$ and $\left.\mathrm{TTT}_{\text {alternative }}\right)$ resulted in sensitivity and specificity values exceeding the arbitrarily chosen cut-off of $80 \%$ (Table 3, and Figure 2).

Table 3. Diagnostic accuracy using receiver operator characteristic (ROC) findings for various combinations of sensory modalities as part of TTT evaluation when comparing SFN versus no SFN patients.

\begin{tabular}{|c|c|c|c|c|c|c|c|c|c|}
\hline \multicolumn{10}{|l|}{ A } \\
\hline \multicolumn{5}{|l|}{ Location } & \multicolumn{5}{|l|}{ Foot } \\
\hline Modality & \multicolumn{3}{|c|}{ Warm } & \multicolumn{3}{|c|}{ Cold } & \multicolumn{3}{|c|}{ Warm + Cold } \\
\hline \multirow[t]{2}{*}{ Method } & Lev & Lim & LeLi & Lev & Lim & LeLi & Lev* & Lim & LeLi \\
\hline & $R / L$ & $R / L$ & $R / L$ & $R / L$ & $R / L$ & $R / L$ & $R / L$ & $R / L$ & $R / L$ \\
\hline AUC-ROC & 0.78 & 0.50 & 0.59 & 0.77 & 0.79 & 0.82 & 0.89 & 0.71 & 0.81 \\
\hline Sensitivity (\%) & 71.6 & 0 & 0 & 59.0 & 68.8 & 66.7 & 82.1 & 26.1 & 45.5 \\
\hline Specificity (\%) & 77.8 & 100 & 100 & 93.8 & 81.5 & 87.5 & 81.3 & 98.8 & 97.5 \\
\hline Correct classified (\%) & 74.7 & 77.9 & 77.9 & 76.6 & 75.2 & 77.2 & 81.7 & 82.7 & 86.3 \\
\hline \multicolumn{10}{|l|}{ B } \\
\hline Location & \multicolumn{9}{|c|}{ Hand } \\
\hline Modality & \multicolumn{3}{|c|}{ Warm } & \multicolumn{3}{|c|}{ Cold } & \multicolumn{3}{|c|}{ Warm + Cold } \\
\hline Method & Lev & Lim & LeLi & Lev & $\operatorname{Lim}$ & LeLi & Lev & Lim & LeLi \\
\hline & $R / L$ & $R / L$ & $R / L$ & $R / L$ & $R / L$ & $R / L$ & $R / L$ & $R / L$ & $\mathrm{R} / \mathrm{L}$ \\
\hline AUC-ROC & 0.76 & 0.75 & 0.77 & 0.69 & 0.55 & 0.62 & 0.79 & 0.71 & 0.74 \\
\hline Sensitivity (\%) & 67.1 & 55.7 & 57.0 & 44.3 & 11.1 & 31.8 & 62.0 & 47.6 & 52.4 \\
\hline Specificity (\%) & 79.0 & 91.4 & 88.9 & 92.6 & 98.8 & 91.4 & 86.4 & 90.1 & 88.9 \\
\hline Correct classified (\%) & 73.1 & 73.8 & 73.1 & 68.8 & 60.4 & 65.3 & 74.4 & 71.5 & 72.9 \\
\hline \multicolumn{10}{|l|}{$\mathrm{C}$} \\
\hline Location & \multicolumn{9}{|c|}{ Foot + Hand } \\
\hline Modality & \multicolumn{3}{|c|}{ Warm } & \multicolumn{3}{|c|}{ Cold } & \multicolumn{3}{|c|}{ Warm + Cold } \\
\hline Method & Lev & $\operatorname{Lim}$ & LeLi & Lev & $\operatorname{Lim}$ & LeLi & & $\operatorname{Lev}^{* *}$ & $\operatorname{Lim}$ \\
\hline & $R / L$ & $\mathrm{R} / \mathrm{L}$ & $\mathrm{R} / \mathrm{L}$ & $\mathrm{R} / \mathrm{L}$ & $R / L$ & $R / L$ & & $R / L$ & $R / L$ \\
\hline AUC-ROC & 0.88 & 0.82 & 0.86 & 0.83 & 0.75 & 0.81 & & 0.95 & 0.86 \\
\hline Sensitivity (\%) & 82.3 & 69.6 & 69.6 & 72.4 & 54.8 & 65.0 & & 84.2 & 75.8 \\
\hline Specificity (\%) & 76.5 & 91.4 & 98.8 & 87.5 & 90.1 & 88.8 & & 93.8 & 86.4 \\
\hline Correct classified (\%) & 79.4 & 86.5 & 92.3 & 80.1 & 74.8 & 78.6 & & 89.1 & 81.2 \\
\hline
\end{tabular}

Lev = method of levels; Lim = method of limits; $R=$ right site; $L=$ left site; LeLi = method of levels + limits combined; AUC-ROC = areas under the receiver operator characteristic curves; correct classification: when compared to original protocol.

Data in bold: sensitivity and specificity values exceeding the arbitrarily chosen cut-off of $80 \%$, classified as * $\mathrm{TTT}_{\text {alternative A }}$ and ** $\mathrm{TTT}_{\text {alternativeB }}$ (see Results)

The first combination ( $\left.\mathrm{TTT}_{\text {alternative } \mathrm{A}}\right)$, measuring warm and cold sensation thresholds by the method of levels bilaterally in the feet, demonstrated sensitivity and specificity of $82.1 \%$ and $81.3 \%$, respectively, with correct classification of $81.7 \%$ of the patients. The second combination (labeled as $\mathrm{TTT}_{\text {alternative }}$ ), measuring warm and cold sensation 
thresholds by the method of levels bilaterally in the foot and hand, showed sensitivity and specificity of $84.2 \%$ and $93.8 \%$, respectively with $89.1 \%$ correct classification. The AUC-ROC findings for $\mathrm{TTT}_{\text {alternativeA }}$ versus $\mathrm{TTT}_{\text {alternative }}$ were compared, and $\mathrm{TTT}_{\text {alternative }}$ differentiated significantly better between SFN ${ }_{\text {def }}$ and SFN ${ }_{\text {neg }}(P$-value: 0.0044) (Figure 2). No combination with the method of limits exceeded the $80 \%$ sensitivity and specificity cut-off.

Figure 2. Comparison between the final 2 most discriminating combinations of temperature threshold testing sensory modalities.

Chapter

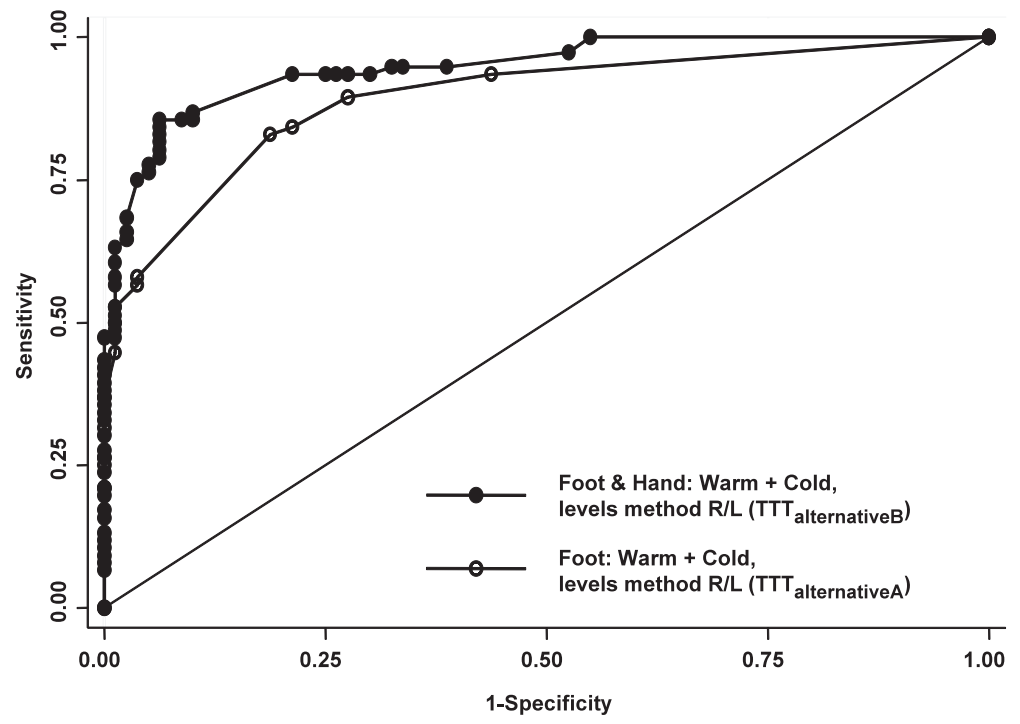

$\mathrm{TTT}_{\text {alternative }}$ was more discriminative than $\mathrm{TTT}_{\text {alternative }}$ when examining patients with small fiber neuropathy $\left(S_{\text {def }}\right)$ versus patients not having SFN $\left(\mathrm{SFN}_{\text {neg }}\right)(p=0.0044) . \mathrm{TTT}_{\text {atternative }}$ : Area under the curve (AUC) 0.89, Standard Error (SE) 0.025, 95\%-confidence interval (95\%-Cl) 0.842-0.942; TTT alternative: : AUC 0.95, SE 0.017, 95\%Cl 0.914-0.980. TTT: temperature threshold testing, R: Right side, L: Left side.

$\mathrm{TTT}_{\text {alternativeB }}$ findings were subsequently compared with the scores obtained when assessing the patients only on the right or left side. The diagnostic accuracy (AUC-ROC) of each one-sided approach was consistently and significantly lower when compared with the same combination assessed on both sides (Figure 3). Furthermore, the onesided values did not reach the predefined cut-off $>80 \%$ (sensitivity, specificity, and correctly classified: right side: $74.7 \%, 93.8 \%$, and $84.3 \%$, respectively; left side: $82.9 \%$, $73.8 \%$, and $78.2 \%$, respectively).

For the modality heat pain, 97-99\% of all patients examined (irrespective of belonging to the SFN def $_{\text {or }} \mathrm{SFN}_{\text {neg }}$ group) demonstrated normal findings, thus heat pain did not differentiate between the 2 groups. 
Figure 3. Discriminatory comparison between the combined (right and left) findings for assessing warm and cold (levels method) in the feet and hands versus one-sided (right or left).

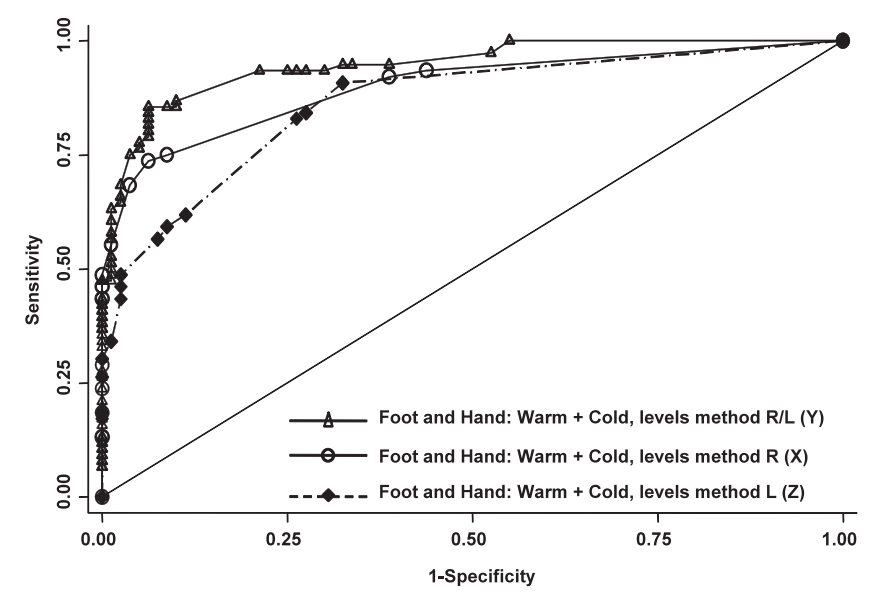

The assessment only on the right side [" $\mathrm{X}^{\prime \prime}$; Area under the curve (AUC) 0.90, Standard Error (SE) 0.024, 95\%-confidence interval (95\%-CI) 0.852-0.947] or on the left side ("Z"; AUC 0.86, SE 0.028, 95\%-CI 0.809-0.919) demonstrated lower discriminative capacities versus the findings when both sides were assessed together ("Y"; AUC 0.95, SE 0.017, 95\%-Cl 0.914-0.980; p<0.0001). R: right side, L: left side.

\section{Examining possible shift in classification of patients and burden reduction for patients}

According to the results of the original TTT protocol (threshold abnormal only if abnormal by method of levels and by method of limits; TTT $_{\text {LevtLim+), }}$, there were 81 $\mathrm{SFN}_{\text {def }}$ patients, $81 \mathrm{SFN}_{\text {neg }}$ patients, and $155 \mathrm{SFN}_{\text {possible }}$ patients. Classifying the patients according to the best alternative approach to the original protocol (TTT aternative $)$ revealed a shift classification in 17 patients, changing from $\mathrm{SFN}_{\text {possible }}$ to $\mathrm{SFN}_{\text {def }}$ and another 45 patients shifting from SFN neg to SFN possible (19.6\% changing classification, see supplementary Table e-1). The remaining patient diagnoses (80.4\%) did not change. Applying the recently published diagnosis of SFN' (defined as typical SFN symptoms/ signs combined with abnormal IENFD or abnormal TTT) resulted in a shift in 45 of 317 patients (14.2\%) from $\mathrm{SFN}_{\text {neg }}$ to $\mathrm{SFN}_{\text {possible. }}$.

By applying the best TTT alterative approach ( $T_{T} T_{\text {atterantive }}$ ), the burden for patients was substantially reduced as the examination time now approximated 40-45 minutes (a reduction of $40-50 \%)$.

\section{DISCUSSION}

The combination of warm and cold sensation thresholds using the method of levels in both feet and hands showed good sensitivity and specificity. It classifed 89\% of the 
patients correctly compared to the original testing protocol that also includes the method of limits evaluation. By doing so, the testing time was reduced substantially, while maintaining acceptable accuracy. Starting the testing protocol in the feet is recommended, since most abnormalities were found in the feet compared to the hands, but testing the hands is mandatory in those with normal thresholds in the feet. Although SFN tends to be symmetrical, bilateral testing warrants recognition of those patients with asymmetrical distribution of SFN. 21,22 $^{2}$

In clinical practice, TTT is often performed using the method of limits, though it is considered to be less accurate since it is reaction time dependent and overestimates threshold values. ${ }^{4,14}$ Comparison between the different methods (levels versus limits) has been described in other studies. Some only highlighted normative values for the different methods, ${ }^{25}$ while others described a correlation between the results of 2 methods as poor to moderate. ${ }^{26-28}$ Repeatability of both methods has been examined, and comparable or better results were obtained using the method of levels. ${ }^{13,15,29}$ Very few have focused on sensitivity of diagnosis using different patient groups and research methods, which limits extrapolation of the results. 13,29,30 A preference for the method of levels has been suggested because no effect of stimulus temperature change rate is seen, ${ }^{31,32}$ and applicability is possible even in subjects who are difficult to examine, such as cognitively impaired subjects and children. ${ }^{25,33}$

Looking at the whole group of patients, $80.4 \%$ retained their previous classification when using the newly-established protocol, and $86 \%$ had no classification change when applying the recently published SFN definition.' These findings appear to be acceptable regarding reduced patient burden, especially since there is no gold standard for the diagnosis of SFN. The time needed to perform the new TTT testing protocol was reduced by $40-45$ minutes (up to $50 \%$ ).

A limitation to this study is the use of a patient cohort suspected to have SFN. Some patients diagnosed as not having SFN may later fulfill the criteria. Also, our center also serves as a tertiary referral center for sarcoidosis, and thus a disproportionate number of subjects were diagnosed with sarcoidosis as a possible etiology for SFN.

Despite these findings, international consensus is needed to standardize the use of TTT as a diagnostic, follow-up, and endpoint tool in patients, because over 15 types of instruments are currently being used worldwide with a variety of methodological approaches for location, stimulus application, and sensation qualities examined. ${ }^{6}$ The findings in our study could serve as the first step towards obtaining such a consensus, pending validation of our findings in other neurophysiology laboratories. Furthermore, future studies should focus on the gold standard for diagnosis SFN.

In conclusion, combining bilateral warm and cold assessment of feet and hands with the method of levels is proposed for future studies. This combination showed the highest acceptable sensitivity and specificity values, substantially reducing patient testing time while maintaining test accuracy. However, international standardization of TTT is still needed. 


\section{REFERENCES}

1. Tesfaye S, Boulton AJ, Dyck PJ, Freeman R, Horowitz M, Kempler P, et al. Diabetic neuropathies: update on definitions, diagnostic criteria, estimation of severity, and treatments. Diabetes Care 2010;33:2285-2293.

2. Devigili G, Tugnoli V, Penza P, Camozzi F, Lombardi R, Melli G, et al. The diagnostic criteria for small fibre neuropathy: from symptoms to neuropathology. Brain 2008;131:1912-1925.

3. Hoeijmakers JG, Faber CG, Lauria G, Merkies IS, Waxman SG. Small-fibre neuropathies--advances in diagnosis, pathophysiology and management. Nat Rev Neurol 2012;8:369-379.

4. Dyck PJ, Zimmerman I, Gillen DA, Johnson D, Karnes JL, O'Brien PC. Cool, warm, and heat-pain detection thresholds: testing methods and inferences about anatomic distribution of receptors. Neurology 1993;43:1500-1508.

5. Shy ME, Frohman EM, So YT, Arezzo JC, Cornblath DR, Giuliani MJ, et al. Quantitative sensory testing: report of the Therapeutics and Technology Assessment Subcommittee of the American Academy of Neurology. Neurology 2003;60:898-904.

6. Bakkers M, Faber CG, Peters MJ, Reulen J, Franssen H, Fischer TZ, et al. Temperature threshold testing: a systematic review. J Peripher Nerv Syst 2013;18:7-18.

7. Rolke R, Baron R, Maier C, Tolle TR, Treede RD, Beyer A, et al. Quantitative sensory testing in the German Research Network on Neuropathic Pain (DFNS): standardized protocol and reference values. Pain 2006;123:231-243.

8. Rolke R, Magerl W, Campbell KA, Schalber C, Caspari S, Birklein F et al. Quantitative sensory testing: a comprehensive protocol for clinical trials. Eur J Pain 2006;10:77-88.

9. Dyck PJ, Larson TS, O'Brien PC, Velosa JA. Patterns of quantitative sensation testing of hypoesthesia and hyperalgesia are predictive of diabetic polyneuropathy: a study of three cohorts. Nerve growth factor study group. Diabetes Care 2000;23:510-517.

10. Singleton JR, Bixby B, Russell JW, Feldman EL, Peltier A, Goldstein J, et al. The Utah Early Neuropathy Scale: a sensitive clinical scale for early sensory predominant neuropathy. J Peripher Nerv Syst 2008;13:218227.

11. Nebuchennykh M, Loseth S, Lindal S, Mellgren SI. The value of skin biopsy with recording of intraepidermal nerve fiber density and quantitative sensory testing in the assessment of small fiber involvement in patients with different causes of polyneuropathy. J Neurol 2009;256:1067-1075.

12 Backonja M, Attal N, Baron R, Bouhassira D, Drangholt M, Dyck PJ, et al. Value of quantitative sensory testing in neurological and pain disorders: NeuPSIG consensus. Pain 2013;154:1807-1819.

13. Kemler MA, Reulen JP, van Kleef M, Barendse GA, van den Wildenberg FA, Spaans F. Thermal thresholds in complex regional pain syndrome type I: sensitivity and repeatability of the methods of limits and levels. Clin Neurophysiol 2000;111:1561-1568.

14. Reulen JP, Lansbergen MD, Verstraete E, Spaans F. Comparison of thermal threshold tests to assess small nerve fiber function: limits vs. levels. Clin Neurophysiol 2003;114:556-563.

15. Yarnitsky D, Sprecher E. Thermal testing: normative data and repeatability for various test algorithms. J Neurol Sci 1994;125:39-45.

16. Yarnitsky D, Sprecher E, Zaslansky R, Hemli JA. Heat pain thresholds: normative data and repeatability. Pain 1995;60:329-332.

17. Hoitsma E, Drent M, Verstraete E, Faber CG, Troost J, Spaans F, et al. Abnormal warm and cold sensation thresholds suggestive of small-fibre neuropathy in sarcoidosis. Clin Neurophysiol 2003;114:2326-2333.

18. Lauria G, Hsieh ST, Johansson O, Kennedy WR, Leger JM, Mellgren SI, et al. European Federation of Neurological Societies/Peripheral Nerve Society Guideline on the use of skin biopsy in the diagnosis of small fiber neuropathy. Report of a joint task force of the European Federation of Neurological Societies and the Peripheral Nerve Society. Eur J Neurol 2010;17:903-912, e944-909.

19. Lauria G, Bakkers M, Schmitz C, Lombardi R, Penza P, Devigili G, et al. Intraepidermal nerve fiber density at the distal leg: a worldwide normative reference study. J Peripher Nerv Syst 2010;15:202-207.

20. Schepers RJ, Ringkamp M. Thermoreceptors and thermosensitive afferents. Neurosci Biobehav Rev 2009;33:205-212.

21. Camdessanche JP, Jousserand G, Ferraud K, Vial C, Petiot P, Honnorat J, et al. The pattern and diagnostic criteria of sensory neuronopathy: a case-control study. Brain 2009;132:1723-1733. 
22. Gorson KC, Herrmann DN, Thiagarajan R, Brannagan TH, Chin RL, Kinsella LJ, et al. Non-length dependent small fibre neuropathy/ganglionopathy. JNNP 2008;79:163-169.

23. Hosmer D, Lemeshow S. Applied logistic regression, Second edition ed. New York: John Wiley \& Sons Inc., 2000.

24. DeLong ER, DeLong DM, Clarke-Pearson DL. Comparing the areas under two or more correlated receiver operating characteristic curves: a nonparametric approach. Biometrics 1988;44:837-845.

25. Meier PM, Berde CB, DiCanzio J, Zurakowski D, Sethna NF. Quantitative assessment of cutaneous thermal and vibration sensation and thermal pain detection thresholds in healthy children and adolescents. Muscle Nerve 2001;24:1339-1345.

26. Fillingim RB, Maddux $V$, Shackelford JA. Sex differences in heat pain thresholds as a function of assessment method and rate of rise. Somatosens Mot Res 1999;16:57-62.

27. Lin YH, Hsieh SC, Chao CC, Chang YC, Hsieh ST. Influence of aging on thermal and vibratory thresholds of quantitative sensory testing. J Peripher Nerv Syst 2005;10:269-281.

28. Defrin R, Shachal-Shiffer M, Hadgadg M, Peretz C. Quantitative somatosensory testing of warm and heat-pain thresholds: the effect of body region and testing method. Clin J Pain 2006;22:130-136.

29. Claus D, Hilz MJ, Neundorfer B. Thermal discrimination thresholds: a comparison of different methods. Acta Neurol Scand 1990;81:533-540.

30. Levy D, Abraham R, Reid G. A comparison of two methods for measuring thermal thresholds in diabetic neuropathy. J Neurol Neurosurg Psychiatry 1989;52:1072-1077.

31. Pertovaara A, Kauppila T, Hamalainen MM. Influence of skin temperature on heat pain threshold in humans. Exp Brain Res 1996;107:497-503.

32. Yarnitsky D, Ochoa JL. Warm and cold specific somatosensory systems. Psychophysical thresholds, reaction times and peripheral conduction velocities. Brain 1991;114 (Pt 4):1819-1826.

33. Defrin R, Pick CG, Peretz C, Carmeli E. A quantitative somatosensory testing of pain threshold in individuals with mental retardation. Pain 2004;108:58-66. 

Chapter 4

\title{
Contact Heat Evoked \\ Potentials: normal values \\ and use in small \\ fiber neuropathy
}

\begin{abstract}
Vera Lagerburg PhD ${ }^{* 1}$, Mayienne Bakkers MD*2, Anne Bouwhuis MD2, Janneke G.J. Hoeijmakers MD, PhD², Arjen M. Smit3, Sofia J.M van den Berg3, Ineke Hordijk-de Boer ${ }^{3}$, Mardien D.G. Brouwer-van der Lee ${ }^{3}$, Douwe Kranendonk MD4, Jos P.H. Reulen PhD', Catharina G. Faber MD, PhD², Ingemar S.J. Merkies MD, PhD

${ }^{*}$ Co-first author
\end{abstract}

'Department of Clinical Physics, Catharina Hospital Eindhoven, the Netherlands 2Department of Neurology Maastricht University Medical Centre, the Netherlands ${ }^{3}$ Department of Clinical Neurophysiology, Spaarne Hospital Hoofddorp, the Netherlands

${ }^{4}$ Department of Neurology Spaarne Hospital Hoofddorp, the Netherlands ${ }^{5}$ Department of Clinical Neurophysiology Maastricht University Medical Centre, the Netherlands 


\section{ABSTRACT}

Introduction: Contact heat-evoked potentials (CHEPs) may be an objective, noninvasive diagnostic tool in small fiber neuropathy (SFN). This study provides CHEP normal values and examines its applicability in SFN patients.

Methods: Standardized CHEPs (Medoc', Ramat Yishai, Israel) were administered at the wrist and ankle. The N2 and P2 latencies and N2-P2-peak-peak-amplitude were recorded through EEG. Healthy subjects (97), stratified by age and gender, and SFN patients with abnormal intraepidermal nerve fiber density (42), were examined. CHEP reproducibility and inter-observer values were investigated.

Results: CHEP normative values were determined. There was a 9-16\% increase in latencies/cm height with increasing age. Amplitudes were higher in women than men, and decreased (17-71\%) with aging. Test-retest reproducibility and interobserver values were $>0.61$ and $>0.96$, respectively. In $73.8 \%$ of the patients, CHEPs were abnormal.

Conclusion: This study provides normal values, reliability, and clinical applicability of CHEPs in SFN. 


\section{INTRODUCTION}

In small fiber neuropathy (SFN), small-caliber myelinated (AS) and unmyelinated (C) fibers are affected. ${ }^{12}$ Interest in this syndrome has increased, particularly since the introduction of intra-epidermal nerve fiber density (IENFD) assessment. ${ }^{3}$ The diagnosis of SFN is based on sensory and autonomic symptoms not otherwise explained, intact large fiber function on examination (normal vibration sense and nerve conduction studies) with abnormal IENFD and/or abnormal temperature threshold testing (TTT).,5 The sensitivity of IENFD for SFN depends highly on chosen cut-off values, ${ }^{6}$ and this technique is not widely available. Furthermore, TTT requires subject cooperation, is potentially vulnerable to malingering, and studies show great variability regarding techniques and methods used? In addition, autonomic function testing can be performed, but it may not be sufficiently sensitive.,.8

Therefore, there is a need for additional objective, reliable, and sensitive diagnostic tools for SFN. Cerebral EEG responses to heat stimuli, conveyed by A $\delta$ and C fibers, can be evoked and measured objectively by laser and contact heat evoked potentials (LEPs and CHEPs). ${ }^{9-12}$ CHEPs have some advantages over LEPs; these include fewer safety precautions, better baseline temperature control, and negligible burn risk. ${ }^{13}$ In SFN, CHEPs appear to be sensitive and to correlate with IENFD. 10,13-16 Previous studies have found CHEP latency and amplitude values to be dependent on gender, age, and pain thresholds. ${ }^{17-20}$ However, normative values are only available for an Asian population. ${ }^{18}$ Therefore, we aimed to provide age- and gender-stratified normative values for CHEPs over a wide age range of healthy Dutch controls. In addition, we examined the reproducibility of the CHEP curves and inter-observer readouts of researchers, and we determined the clinical applicability of CHEPs in patients with SFN and abnormal IENFD.

\section{METHODS}

\section{Healthy controls}

The study took place between January 2009 and February 2013. All data were collected prospectively. Healthy participants were recruited from hospital volunteers and through advertisements. Examination of all participants took place at outpatient clinic departments in a comfortable, temperature-controlled room. All participants underwent a standardized interview and examination. Eligibility was based on the following: age 18 years or older, no sensory symptoms or signs on neurological examination, no diseases that may cause polyneuropathy [e.g., diabetes mellitus, renal failure, alcohol abuse (arbitrarily defined as drinking at least 4 international units per day), systemic illnesses like sarcoidosis or malignancy], no past or present use of neurotoxic drugs, and normal nerve conduction studies. 


\section{Patients}

Patients with a distal sensory neuropathy compatible with SFN were recruited at the Maastricht University Medical Centre (MUMC). Exclusion criteria included limb or spine deformities, other neurological diseases, and symptoms or signs of large fiber neuropathy such as weakness, vibration threshold abnormalities as determined by Rydel-Seiffer graduated tuning fork, or abnormalities on nerve conduction studies in either latency, amplitude, or velocity of 2 or more examined nerves.

In the absence of a gold standard for the diagnosis of SFN, patients with SFN complaints and abnormal IENFD compared to reference values, ${ }^{21}$ were selected as a validation cohort, since we aimed to use the abnormal IENFD as a surrogate "gold standard" in order to determine the sensitivity of the various CHEP parameters.

\section{Assessments}

Basic requirements and assessments

Basic characteristics (age, gender, and height) were assessed, and a standardized neurological examination was performed. Nerve conduction studies were performed with a Keypoint ${ }^{\circ}$.NET (Medtronic, Minneapolis, USA) or Synergy ${ }^{\mathrm{TM}}$ (Natus Medical Inc, Middleton, USA) machine. One-sided measurement of compound muscle action potential (CMAP) or sensory nerve action potential (SNAP) amplitude and nerve conduction velocity of median motor and sensory nerves, fibular motor, tibial motor, and sural sensory nerves was conducted in patients. F-responses of median, fibular, and tibial nerves were recorded as well as a tibial H-reflexes. In healthy subjects, only fibular CMAP amplitude and sural SNAP amplitude and nerve conduction velocity were measured to minimize subject discomfort. Heat pain (healthy subjects and patients) and warm and cool thresholds (patients) were determined at the thenar eminence and dorsum of the foot with the Advanced Thermal Stimulator or the Thermal Sensory Analyzer (Medoc', Ramat Yishai, Israel) in accordance with available guidelines and compared to published normative data. ${ }^{22-24}$ Heat pain threshold was determined by the method of limits. Warm and cool thresholds were considered abnormal if they were outside reference values for both the method of levels and limits.

In patients, a skin biopsy was performed in the lower leg for IENFD determination, as previously described. ${ }^{5,25}$

Contact Heat Evoked Potentials (CHEPs)

A Contact Heat Evoked Potential Stimulator (Medoc', Ramat Yishai, Israel) was used to apply heat pulses to the distal volar side of the right forearm and $10 \mathrm{~cm}$ above the right lateral malleolus. The thermode consists of a heating thermo foil and a Peltier element with an active water cooling system, heating and cooling rate $70{ }^{\circ} \mathrm{C}$ and 40 ${ }^{\circ} \mathrm{C}$ /second respectively, and thermode diameter of $29 \mathrm{~mm}$. Baseline temperature was set at $35{ }^{\circ} \mathrm{C}$, peak temperature at $51{ }^{\circ} \mathrm{C}$. Evoked potentials were recorded using the Keypoint. NET (Medtronic, Minneapolis, USA) or through continuous EEG recording (Brainlab 4.0 OSG, Belgium), with the electrodes placed on Cz, Pz, and Fz according 
to the international 10-20 system, referenced to linked ears. The evoked potentials were filtered with a band pass filter at 0.2 - $100 \mathrm{~Hz}$. With the Keypoint ${ }^{\circ}$.NET, artifact rejection was automatic, based on the amplitude of the response. Two test stimuli were given to exclude startle phenomena (e.g. blink, muscle, and movement artifacts). A pilot study of 15 participants determined how many stimuli would be tolerable for a subject and could still be averaged for a reliable response. Per location (first wrist, then ankle) a series of 12 stimuli was given. The participants were instructed to keep their eyes open in a fixed, neutral position to avoid blink artifacts and EEG alpha wave contamination during the time frame of the stimulus. The inter-stimulus interval was set randomly between 10 and $18 \mathrm{sec}$. To avoid habituation to the heat stimuli, the thermode was moved slightly after each stimulus, remaining within a predefined area (similar distance to recording EEG electrode), and maintaining optimal skin contact.26,

${ }^{27}$ All subjects were tested twice within a visit with a time interval of approximately 15-30 minutes.

Protocol approval, registration, and consent

The study was approved by the medical ethics committee of the participating hospital and the Central Committee for Human Related Research (identifier number p06.0066L/MEC 05-224) in accordance with the guidelines of the Declaration of Helsinki (amendment October 2008, Seoul, Korea). All participants gave written informed consent prior to inclusion.

\section{Statistical analysis}

Assessment of CHEP parameters

Latency of the first negative $\left(N_{2}\right)$ and positive $\left(P_{2}\right)$ peaks and $N_{2}-P_{2}$ peak-to-peak amplitude of the late evoked responses (at Cz) were assessed in all participants per testing site and per test run. For all healthy subjects the $\mathrm{N}_{2}$ - and $\mathrm{P}_{2}$-latencies and $\mathrm{N}_{2}$ $P_{2}$ amplitudes of the averaged recordings were determined by visual inspection by 2 observers (physicist and technician).The first run was used to calculate normative values using quantile regression analyses (95\% chosen specificity) and for comparison purposes between the CHEP parameters. The possible influence of age, gender, and height were also examined. The $95^{\text {th }}$ percentile upper limits and median values were estimated for the $\mathrm{N}_{2}$ and $\mathrm{P}_{2}$-latencies. The $5^{\text {th }}$ percentile lower limit and median values were calculated for the $\mathrm{N}_{2}-\mathrm{P}_{2}$ amplitudes on both sides examined. Comparison of basic scores between healthy controls and patients with SFN was performed using a Chisquare test or a Student $t$-test depending on the type of data.

Reproducibility and Receiver Operator Characteristic (ROC) curve studies

Test-retest values were determined for the 2 test runs (test-retest of curve reproducibility) within each session in all healthy subjects and inter-observer readouts (the second assessment was performed without having access to the first data findings), were also calculated (one-way analysis-of-variance).

Patients with SFN and abnormal IENFD were selected as the "validation gold standard 
cohort" for the purposes of this study. Through univariate logistic regression studies and calculation of the corresponding areas under the ROC curves (ROC-AUC) the diagnostic accuracy of the various CHEP parameters was evaluated. Sensitivity (fraction of positive cases classified correctly) and specificity (fraction of negative cases classified correctly) were also calculated to determine which CHEP parameter $\left(\mathrm{N}_{2}-\right.$ latency, $\mathrm{P}_{2}$-latency, $\mathrm{N}_{2}-\mathrm{P}_{2}$ amplitude at the wrist or ankle) best differentiated between healthy controls and the validation patient cohort, and whether acceptable AUC values would be obtained. The ROC-AUC demonstrates no discrimination if values are $\leq 0.5$, acceptable discrimination for values 0.7-0.8, excellent discrimination if values are 0.80.9 , and outstanding discrimination if values are $\geq 0.9 .{ }^{28}$ The ROC-AUCs for the forearm and for the lower leg parameters were compared separately with each other through a test for equality. ${ }^{29}$ Analyses were performed with Stata 11.0 for windows XP (StataCorp LP, College Station, TX). A value of $P<0.05$ was considered significant.

\section{RESULTS}

\section{Participants}

All healthy participants (97) were examined in the outpatient and electrophysiology departments. The total examination took 60-120 minutes. The CHEP procedure was well tolerated by all but 1 healthy participant. This subject withdrew from the study. The basic characteristics for the healthy volunteers are listed in Table 1.

Table 1. General characteristics of healthy participants and patients with small fiber neuropathy (SFN).

\begin{tabular}{|c|c|c|c|}
\hline & $\begin{array}{l}\text { Healthy participants } \\
\qquad(\mathrm{n}=97)\end{array}$ & $\begin{array}{c}\text { SFN patients with abnormal } \\
\text { intraepidermal nerve fiber } \\
\text { density (IENFD) } \\
(\mathrm{n}=42)\end{array}$ & $\begin{array}{c}P \text {-values } \\
\left(X^{2} \text {-test or Student }\right. \\
t \text {-test })\end{array}$ \\
\hline \multicolumn{4}{|l|}{ Gender, n (\%) } \\
\hline Women & $51(52.6)$ & $27(64.3)$ & 0.17 \\
\hline Men & $46(47.4)$ & $15(35.7)$ & \\
\hline Age, mean in Years (SD) range & $44.3(14.5) 17-78$ & 50.6 (12.6) 26-77 & 0.008 \\
\hline Height, mean in cm (SD) range & $176(10.2)$ 150-198 & $174(7.2) 158-194$ & 0.13 \\
\hline \multicolumn{4}{|l|}{$\begin{array}{l}\text { CHEP } \mathrm{N}_{2} \text {-latency, } \\
\text { mean msec }(\mathrm{SD}) \text { range }\end{array}$} \\
\hline Volar forearm & 399 (45) 314-604 & 427 (66) 290-668 & 0.002 \\
\hline Distal leg & 469 (40) 322-569 & $512(65) 418-708$ & $<0.0001$ \\
\hline \multicolumn{4}{|l|}{$\begin{array}{l}\mathrm{CHEP}_{2} \text {-latency, } \\
\text { mean msec (SD) range }\end{array}$} \\
\hline Volar forearm & $522(55) 370-754$ & 534 (79) 378-872 & 0.16 \\
\hline Distal leg & 589 (47) 477-714 & 613 (66) 466-882 & 0.008 \\
\hline \multicolumn{4}{|l|}{$\begin{array}{l}\text { CHEP } \mathrm{N}_{2}-\mathrm{P}_{2} \text { Amplitude } \\
\text { Mean } \mu V(\mathrm{~S} D) \text { range }\end{array}$} \\
\hline Volar forearm & 29.5 (11) 9.2-61.7 & 19.4 (10) 3.7-43 & $<0.0001$ \\
\hline Distal leg & 28.9 (12) 9.6-70.9 & 14.4 (13) $1.9-60.7$ & $<0.0001$ \\
\hline
\end{tabular}


Stratification according to age and gender led to 6 age groups: age 18-29 (9 men, 9 women); 30-39 (7 men, 12 women); 40-49 (12 men, 9 women); 50-59 (11 men, 10 women); 60-69 (5 men, 7 women); 70-79 (3 men, 3 women). Most volunteers were white/Caucasian (98\%). Mean pain thresholds at the foot were $45.9^{\circ} \mathrm{C}\left(\mathrm{SD} 3.0^{\circ} \mathrm{C}, 33.4-\right.$ $50.1^{\circ} \mathrm{C}$ ) which is within the normal range..$^{23}$

A total of 166 patients were examined at our outpatient clinic during the study period, and 42 (25.3\%) had abnormal IENFD. There were more women in the patient group compared to controls, but this was not significant (Table 1). The mean age was higher in patients compared to controls. All CHEP parameters except $P_{2}$-Latency at the forearm differed significantly between the control and patient groups (Table 1).

\section{CHEP results and Reproducibility}

Evoked potentials could be recorded by stimuli applied to the volar side of the forearm and the lateral ankle $\left(35^{\circ} \mathrm{C}\right.$ baseline temperature) in all except 9 healthy subjects (9\%). Overall, amplitudes were lower in the second session. A stimulus response could be recorded in all but two unresponsive subjects aged 64 and 70 years after having raised the thermode baseline temperature to $40{ }^{\circ} \mathrm{C}$. Latencies were significantly heightdependent, being longer in men compared to women (Figure 1).

Figure 1. CHEP Peak latency and amplitude findings in healthy controls
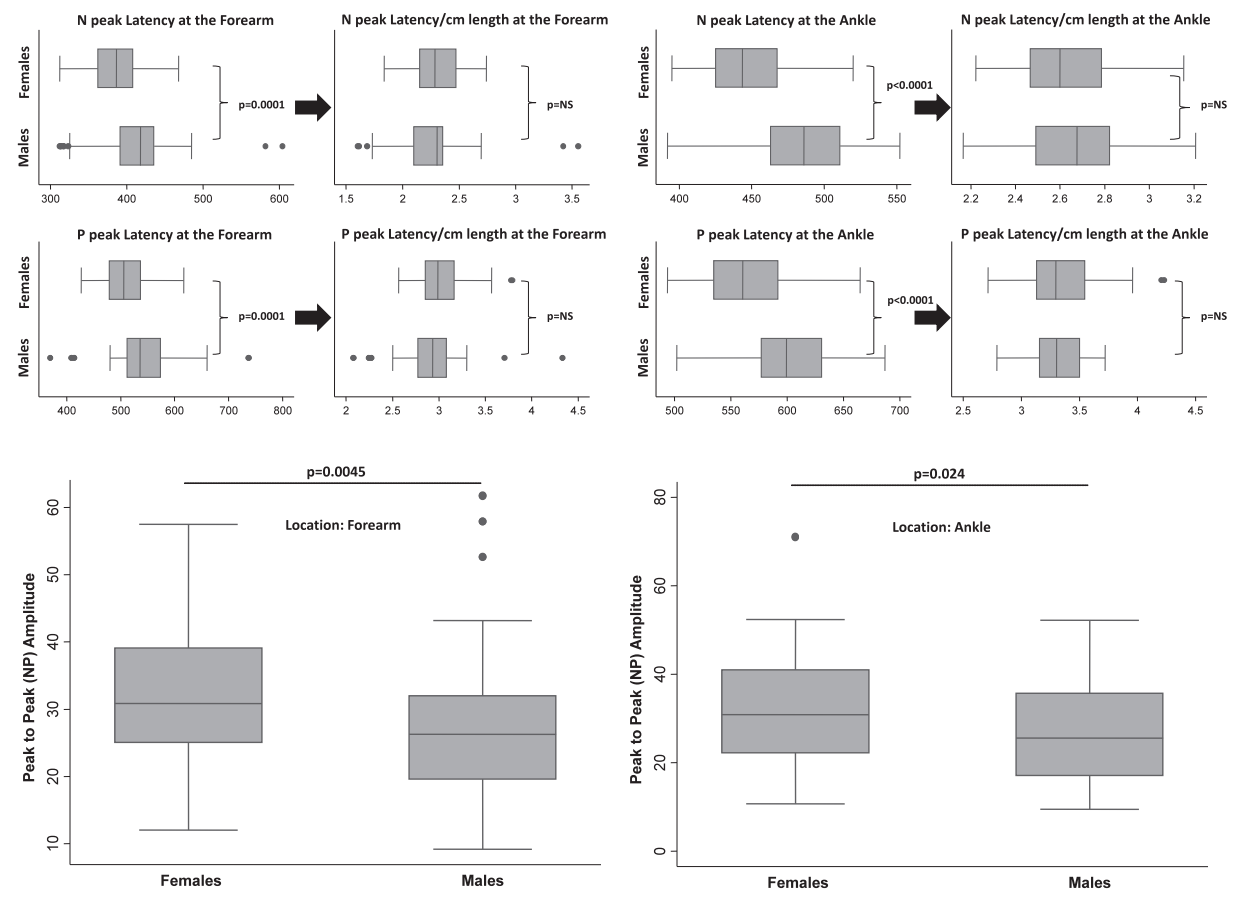

Box plots show peak latencies and peak amplitudes in healthy men and women subjects. NS= not significant. 
By dividing the latencies by height $(\mathrm{cm})$ in each healthy control, we were able to create latency/cm height values that were not gender-dependent; latencies/cm height increased slightly with age. $\mathrm{N}_{2}-\mathrm{P}_{2}$ amplitude values were significantly higher in women than in men and decreased significantly with age (Table 2 and Figure 1).

Normative clinically useful reference values (median and $95^{\text {th }}$ percentiles for the $\mathrm{N}_{2}$ - and $\mathrm{P}_{2}$-latencies/cm height values and median and $5^{\text {th }}$ percentiles for the $\mathrm{N}_{2}-\mathrm{P}_{2}$ amplitudes scores) were calculated from the recorded evoked responses for the 2 examination sites (Table 2). Acceptable to high test-retest curve reproducibility and inter-observer readout reliability scores were obtained with all scores being > 0.6 up to 0.99 (Table 3).

Table 2. Contact heat evoked potential normative values.

\begin{tabular}{|c|c|c|c|c|c|c|c|c|}
\hline \multirow[t]{3}{*}{ A } & \multicolumn{8}{|c|}{ LATENCY Normative Values for MEN AND WOMEN } \\
\hline & \multicolumn{4}{|c|}{ FOREARM } & \multicolumn{4}{|c|}{ LOWER LEG } \\
\hline & \multicolumn{2}{|c|}{$\begin{array}{l}\mathrm{N}_{2} \text {-latency/cm height } \\
(\mathrm{msec} / \mathrm{cm})\end{array}$} & \multicolumn{2}{|c|}{$\begin{array}{l}P_{2} \text {-latency/cm height } \\
(\mathrm{msec} / \mathrm{cm})\end{array}$} & \multicolumn{2}{|c|}{$\begin{array}{l}\mathrm{N}_{2} \text {-latency/cm height } \\
(\mathrm{msec} / \mathrm{cm})\end{array}$} & \multicolumn{2}{|c|}{$\begin{array}{l}P_{2} \text {-latency/cm height } \\
(\mathrm{msec} / \mathrm{cm})\end{array}$} \\
\hline Age & median & \begin{tabular}{|c|}
$95^{\text {th }}$ \\
percentiles
\end{tabular} & median & $\begin{array}{c}95^{\text {th }} \\
\text { percentiles }\end{array}$ & median & $\begin{array}{c}95^{\text {th }} \\
\text { percentiles }\end{array}$ & median & $\begin{array}{c}95^{\text {th }} \\
\text { percentiles }\end{array}$ \\
\hline$<30$ & 2.2 & 2.5 & 2.9 & 3.3 & 2.5 & 2.9 & 3.2 & 3.6 \\
\hline $30-39$ & 2.2 & 2.5 & 2.9 & 3.4 & 2.6 & 3.0 & 3.3 & 3.7 \\
\hline $40-49$ & 2.3 & 2.6 & 3.0 & 3.6 & 2.7 & 3.0 & 3.3 & 3.8 \\
\hline $50-59$ & 2.3 & 2.7 & 3.0 & 3.7 & 2.7 & 3.1 & 3.3 & 3.8 \\
\hline $60-69$ & 2.3 & 2.7 & 3.0 & 3.8 & 2.8 & 3.2 & 3.4 & 3.9 \\
\hline $70+$ & 2.4 & 2.8 & 3.1 & 4.0 & 2.9 & 3.3 & 3.4 & 3.9 \\
\hline
\end{tabular}

B

\begin{tabular}{|c|c|c|c|c|c|c|c|c|}
\hline & \multicolumn{4}{|c|}{ WOMEN } & \multicolumn{4}{|c|}{ MEN } \\
\hline & \multicolumn{2}{|c|}{ WRIST } & \multicolumn{2}{|c|}{ ANKLE } & \multicolumn{2}{|c|}{ WRIST } & \multicolumn{2}{|c|}{ ANKLE } \\
\hline & \multicolumn{2}{|c|}{$\begin{array}{c}\mathrm{N}_{2}-\mathrm{P}_{2} \text { amplitude } \\
(\mu \mathrm{V})\end{array}$} & \multicolumn{2}{|c|}{$\begin{array}{c}\mathrm{N}_{2}-\mathrm{P}_{2} \text { amplitude } \\
(\mu \mathrm{V})\end{array}$} & \multicolumn{2}{|c|}{$\begin{array}{c}\mathrm{N}_{2}-\mathrm{P}_{2} \text { amplitude } \\
(\mu \mathrm{V})\end{array}$} & \multicolumn{2}{|c|}{$\begin{array}{c}\mathrm{N}_{2}-\mathrm{P}_{2} \text { amplitude } \\
(\mu \mathrm{V})\end{array}$} \\
\hline Age & $\begin{array}{c}5^{\text {th }} \\
\text { percentiles }\end{array}$ & median & $\begin{array}{c}5^{\text {th }} \\
\text { percentiles }\end{array}$ & median & $5^{\text {th }}$ percentiles & median & $\begin{array}{c}5^{\text {th }} \\
\text { percentiles }\end{array}$ & median \\
\hline$<30$ & 20.6 & 33.7 & 17.6 & 38.2 & 11.8 & 27.8 & 18.6 & 32.4 \\
\hline 30-39 & 16.4 & 32.5 & 15.2 & 32.4 & 11.4 & 26.8 & 15.2 & 29.1 \\
\hline $40-49$ & 12.8 & 31.4 & 13.2 & 27.4 & 11.1 & 26 & 12.2 & 26.3 \\
\hline 50-59 & 9.3 & 30.5 & 11.1 & 22.4 & 10.8 & 25.1 & 9.2 & 23.4 \\
\hline $60-69$ & 6.1 & 29.5 & 9.3 & 17.8 & 10.5 & 24.3 & 6.5 & 20.9 \\
\hline $70+$ & 1.2 & 28.1 & 6.5 & 11.1 & 10.1 & 23.2 & 2.5 & 17.1 \\
\hline
\end{tabular}

Table 3. Test-retest and inter-observer values of contact heat evoked potential parameters.

\begin{tabular}{|c|c|c|c|c|}
\hline & \multicolumn{2}{|c|}{ WRIST } & \multicolumn{2}{|c|}{ ANKLE } \\
\hline & $\begin{array}{c}\text { test-retest } \\
\text { curves }(95 \%-\mathrm{Cl})\end{array}$ & $\begin{array}{l}\text { Inter-observer } \\
\text { values }(95 \%-C l)\end{array}$ & $\begin{array}{l}\text { test-retest } \\
\text { curves } \\
(95 \%-C l)\end{array}$ & $\begin{array}{c}\text { Inter-observer } \\
\text { values } \\
(95 \%-C l)\end{array}$ \\
\hline $\mathrm{N}_{2}$-latency observer 1 & $0.78(0.69-0.87)$ & \multirow{2}{*}{$\begin{array}{c}\mathrm{N}_{2} \text {-latency } \\
0.96(0.95-0.97)\end{array}$} & $0.66(0.53-0.80)$ & \multirow{2}{*}{$\begin{array}{c}\mathrm{N}_{2} \text {-latency } \\
0.99(0.98-0.99)\end{array}$} \\
\hline $\mathrm{N}_{2}$-latency observer 2 & $0.73(0.63-0.84)$ & & $0.68(0.54-0.81)$ & \\
\hline P-latency observer 1 & $0.75(0.65-0.85)$ & \multirow{2}{*}{$\begin{array}{c}P_{2} \text {-latency } \\
0.97(0.96-0.98)\end{array}$} & $0.62(0.46-0.77)$ & \multirow{2}{*}{$\begin{array}{c}P_{2} \text {-latency } \\
0.97(0.95-0.98)\end{array}$} \\
\hline$P_{2}$-latency observer 2 & $0.74(0.63-0.84)$ & & $0.61(0.46-0.77)$ & \\
\hline $\mathrm{N}_{2}-\mathrm{P}_{2}$ amplitude obs. 1 & $0.67(0.55-0.80)$ & \multirow{2}{*}{$\begin{array}{c}\mathrm{N}_{2}-\mathrm{P}_{2} \text { amplitude } 0.99 \\
(0.99-1.0)\end{array}$} & $0.68(0.54-0.81)$ & \multirow{2}{*}{$\begin{array}{c}\mathrm{N}_{2}-\mathrm{P}_{2} \text { amplitude } 0.99 \\
0.99(0.99-1.0)\end{array}$} \\
\hline $\mathrm{N}_{2}-\mathrm{P}_{2}$ amplitude obs. 2 & $0.67(0.55-0.80)$ & & $0.67(0.53-0.80)$ & \\
\hline
\end{tabular}


Figure 2. Comparison of CHEP parameters at the forearm and ankle separately, using the area under the ROC curves.
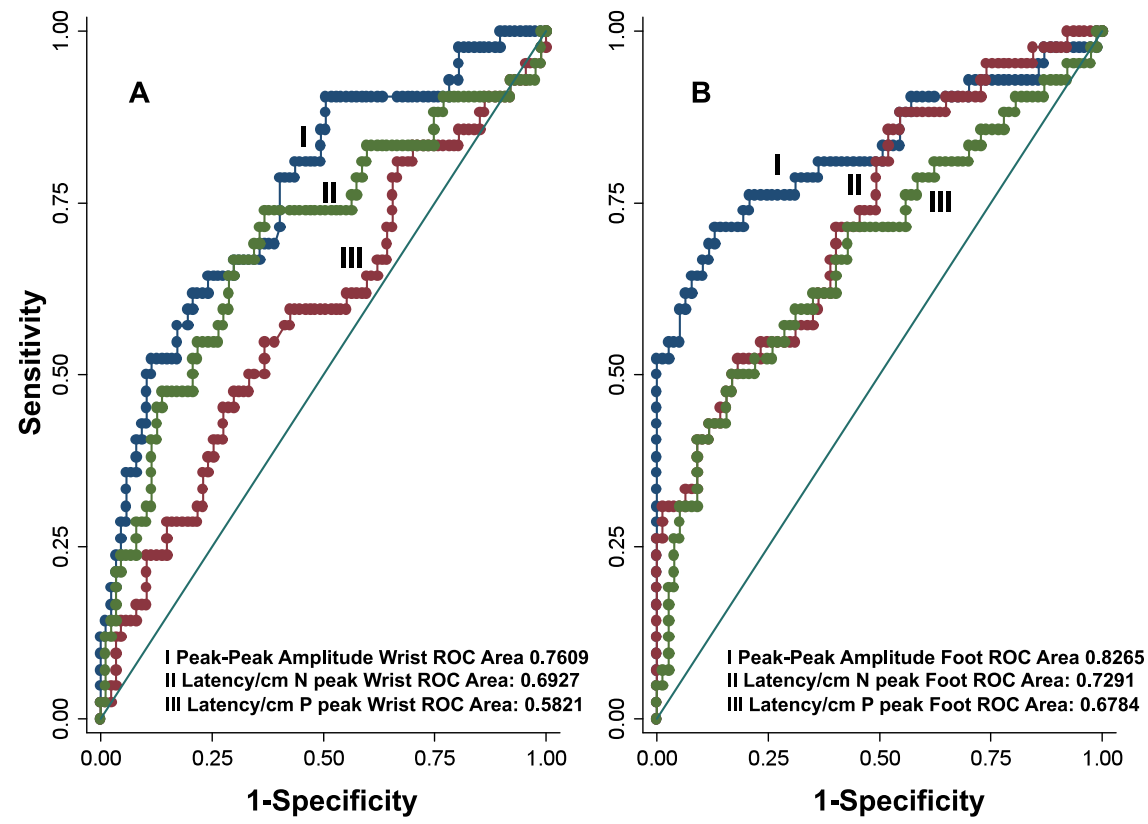

Chapter

A. CHEP parameter comparison at the wrist: the N2-P2 amplitude ROC-AUC was larger (0.7609) when compared to the AUC for N2 peak latency/cm (0.6927) and P2 peak latency/cm height (0.5821), respectively. Through ROC comparison (using Stata command "Roccomp"), we found a significant difference between the amplitude ROC-AUC values versus P2 peak latency/cm height scores ( $P=0.012$ ). No significant difference $(P=0.27)$ was obtained between the N2-P2 amplitude versus N2 peak latency/cm height values. B. CHEP parameter comparison at the ankle: the N2-P2 amplitude ROC-AUC was larger (0.8265) when compared to the AUC for N2 peak latency/cm (0.7291) and P2 peak latency/cm height (0.6784), respectively. The area under the ROC curve for N2-P2 amplitude yielded a favorable trend when compared with the other 2 methods (N2-P2 amplitude versus N2-Latency/cm: $X^{2}=3.63 ; P=0.056 ;$ N2-P2 Amplitude versus P2-Latency/ $\left.\mathrm{cm}: X^{2}=4.83 ; P=0.028\right)$. However, the differences were not significant after Bonferroni correction.

\section{Diagnostic accuracy and comparison between the various CHEP parameters}

The 42 patients with abnormal IENFD were considered the surrogate "gold standard" for calculation of sensitivity, specificity, and percentage of subjects correctly classified. Figure 2 shows the comparison of CHEP parameters ( $\mathrm{N}_{2}$-latency/cm height, $\mathrm{P}_{2}$-latency/ $\mathrm{cm}$ height, and $\mathrm{N}_{2}-\mathrm{P}_{2}$ amplitude) at the forearm and at the ankle, separately. The $\mathrm{N}_{2}-$ $\mathrm{P}_{2}$ amplitude at the ankle demonstrated the highest ROC-AUC (AUC: 0.83; sensitivity 69.1\%, specificity; $87 \%$; correctly classified: $80.7 \%$ ) followed by the $\mathrm{N}_{2}-\mathrm{P}_{2}$ amplitude at the wrist (AUC: 0.76 ; sensitivity 50\%; specificity $88.5 \%$; correctly classified $76 \%$ ). For the remaining parameters, the sensitivity, specificity, and correctly classified findings were lower (data not shown). Comparison between the $\mathrm{N}_{2}-\mathrm{P}_{2}$ amplitude values with the $\mathrm{N}_{2}$ - 
latency/cm and $\mathrm{P}_{2}$-latency/cm at the ankle demonstrated higher values for the $\mathrm{N}_{2}-\mathrm{P}_{2}$ amplitudes (Figure 2). Comparison between the $\mathrm{N}_{2}-\mathrm{P}_{2}$ amplitude values at the ankle versus the values at the wrist showed a trend in favor of the $\mathrm{N}_{2}-\mathrm{P}_{2}$ amplitude at the ankle, but the difference was not significant $(p=0.16)$. Using normative values for the TTT and CHEPs, a diagnostic accuracy of 73.8\% (31/42) was seen for both diagnostic techniques separately in patients with SFN with reduced IENFD. In 60\% of patients (25/42), both TTT and CHEPs were abnormal, and combining both techniques, 88\% (37/42) of patients show an abnormal test result.

\section{DISCUSSION}

This study provides clinically applicable normative values for various CHEP parameters recorded at the volar forearm and distal leg for normal Dutch volunteers. Observed amplitudes and latencies were comparable to reported values. ${ }^{13,17,18,30}$ Subject age showed a slight increase in latency/cm height and a substantial decrease in $\mathrm{N}_{2}-\mathrm{P}_{2}$ amplitude. Similar findings have been reported in a smaller Asian cohort. ${ }^{18}$ Since repeated stimulation at the same location may lead to amplitude reduction, ${ }^{31}$ we randomized the stimulus interval and moved the thermode, as has been advised in previous studies. ${ }^{26,27}$ This optimizes "stimulus novelty", which has been shown to influence amplitudes in laser evoked potentials. ${ }^{32}$ Nonetheless, amplitudes decreased in our subjects after repeated stimulation, suggesting central habituation, ${ }^{33}$ and/or nociceptor fatigue. ${ }^{34}$

This study also provides acceptable test-retest reproducibility for the curves and interobserver reliability values. Others have shown reproducibility in subjects tested twice (on average one month interval) to be "fair to substantial".35

Using our normative CHEP values, versus reported normative TTT values, ${ }^{23,}{ }^{24}$ both techniques capture $74 \%$ of patients with SFN and abnormal IENFD. In addition, CHEP $\mathrm{N}_{2}-\mathrm{P}_{2}$ amplitudes at the ankle more than at the wrist showed acceptable diagnostic accuracy in differentiating between our controls and SFN patients, with reasonable sensitivity and specificity scores. Several studies have demonstrated increased CHEP latencies and reduced amplitudes in various forms of peripheral neuropathies that involve small nerve fibers. ${ }^{10,16,36,37}$ Our ROC findings (Figure 2) and reproducibility values provide further support for CHEPs to be considered as a reliable non-invasive technique to investigate function of small nerve fibers, particularly since skin biopsy is not available widely.

There are some methodological issues that should be addressed. First, recordings were not obtained initially in 9 healthy subjects (9\%). However, after a slight increase of baseline temperature to $40^{\circ} \mathrm{C}$, there were only 2 subjects (2\%) in whom no CHEPs were obtained. Similar observations were reported in previous studies. ${ }^{17,27,38,39}$ A possible partial explanation for this effect could be that at higher temperature, the ultra-late 
C-fiber response does not influence the occurrence of the late $A \delta$ response. ${ }^{40}$ Due to the burden of the CHEP examination, no additional studies were performed at $40^{\circ} \mathrm{C}$ for the responsive subjects examined already examined at $35^{\circ} \mathrm{C}$. However, future studies should determine the best baseline temperature and optimum number of stimuli to improve test performance. Also, the question remains open whether some of these 'healthy' subjects may have subclinical nerve fiber deficits as a form of subclinical polyneuropathy. Secondly, we had difficulty recruiting healthy subjects aged $>70$ years. Most potential participants in this age category were rejected due to comorbidity and/or abnormalities on examination. Some caution is therefore suggested when using the normative values of subjects over age 70 years. Thirdly, the first CHEP responses obtained after the test stimuli were often the clearest responses. On the other hand, subjects did not usually react with movements to the stimulus, and it was very easy to avoid blinking during the actual delivery of the heat pulse. So, giving test stimuli and discarding the results of these stimuli to exclude "startle effects", may have negatively influenced the results. Fourth, a diagnostic accuracy of 74\% was obtained with the CHEPs in SFN patients diagnosed using abnormal IENFD as a surrogate "gold standard", but a lower sensitivity could be expected in an unselected SFN population, since skin biopsy does not capture all SFN patients.

Despite these shortcomings, this study presents clinically applicable, reliable normative values for CHEPs in the distal leg and volar forearm and demonstrates its clinical utility in patients with SFN. The use of CHEPs is suggested as an additional non-invasive tool in the diagnosis of SFN. 


\section{REFERENCES}

1. Gorson KC, Ropper AH. Idiopathic distal small fiber neuropathy. Acta Neurol Scand 1995;92(5):376-382.

2. Stewart JD, Low PA, Fealey RD. Distal small fiber neuropathy: results of tests of sweating and autonomic cardiovascular reflexes. Muscle Nerve 1992;15(6):661-665.

3. McCarthy BG, Hsieh ST, Stocks A, Hower P, Macko C, Cornblath DR et al. Cutaneous innervation in sensory neuropathies: evaluation by skin biopsy. Neurology 1995;45(10):1848-1855.

4. Tesfaye S, Boulton AJ, Dyck PJ, Freeman R, Horowitz M, Kempler P et al. Diabetic neuropathies: update on definitions, diagnostic criteria, estimation of severity, and treatments. Diabetes care 2010;33(10):22852293.

5. Lauria G, Hsieh ST, Johansson O, Kennedy WR, Leger JM, Mellgren SI et al. European Federation of Neurological Societies/Peripheral Nerve Society Guideline on the use of skin biopsy in the diagnosis of small fiber neuropathy. Report of a joint task force of the European Federation of Neurological Societies and the Peripheral Nerve Society. Eur J Neurol 2010;17(7):903-912, e944-949.

6. Nebuchennykh M, Loseth S, Lindal S, Mellgren SI. The value of skin biopsy with recording of intraepidermal nerve fiber density and quantitative sensory testing in the assessment of small fiber involvement in patients with different causes of polyneuropathy. J Neurol 2009;256(7):1067-1075.

7. Bakkers M, Faber CG, Peters MJ, Reulen JP, Franssen H, Fischer TZ et al. Temperature threshold testing: a systematic review. J Peripher Nerv Syst 2013;18(1):7-18.

8. Le Quesne PM, Fowler CJ, Parkhouse N. Peripheral neuropathy profile in various groups of diabetics. J Neurol Neurosurg Psychiatry 1990;53(7):558-563.

9. Le Pera D, Valeriani M, Niddam D, Chen AC, Arendt-Nielsen L. Contact heat evoked potentials to painful and non-painful stimuli: effect of attention towards stimulus properties. Brain Topogr 2002;15(2):115123.

10. Chao CC, Hsieh SC, Tseng MT, Chang YC, Hsieh ST. Patterns of contact heat evoked potentials (CHEP) in neuropathy with skin denervation: correlation of CHEP amplitude with intraepidermal nerve fiber density. Clin Neurophysiol 2008;119(3):653-661.

11. Magerl W, Ali Z, Ellrich J, Meyer RA, Treede RD. C- and A delta-fiber components of heat-evoked cerebral potentials in healthy human subjects. Pain 1999;82(2):127-137.

12. Truini A, Galeotti F, Romaniello A, Virtuoso M, lannetti GD, Cruccu G. Laser-evoked potentials: normative values. Clin Neurophysiol 2005;116(4):821-6.

13. Atherton DD, Facer P, Roberts KM, Misra VP, Chizh BA, Bountra C et al. Use of the novel Contact Heat Evoked Potential Stimulator (CHEPS) for the assessment of small fiber neuropathy: correlations with skin flare responses and intra-epidermal nerve fiber counts. BMC neurology 2007;7:21.

14. Chen AC, Niddam DM, Arendt-Nielsen L. Contact heat evoked potentials as a valid means to study nociceptive pathways in human subjects. Neuroscience letters 2001;316(2):79-82.

15. Casanova-Molla J, Grau-Junyent JM, Morales M, Valls-Sole J. On the relationship between nociceptive evoked potentials and intraepidermal nerve fiber density in painful sensory polyneuropathies. Pain 2011;152(2):410-418.

16. Chao CC, Tseng MT, Lin YJ, Yang WS, Hsieh SC, Lin YH et al. Pathophysiology of neuropathic pain in type 2 diabetes: skin denervation and contact heat-evoked potentials. Diabetes Care 2010;33(12):2654-2659.

17. Chen IA, Hung SW, Chen YH, Lim SN, Tsai YT, Hsiao CL et al. Contact heat evoked potentials in normal subjects. Acta Neurol Taiwan 2006;15(3):184-191.

18. Chao CC, Hsieh ST, Chiu MJ, Tseng MT, Chang YC. Effects of aging on contact heat-evoked potentials: the physiological assessment of thermal perception. Muscle Nerve 2007;36(1):30-38.

19. Truini A, Galeotti F, Pennisi E, Casa F, Biasiotta A, Cruccu G. Trigeminal small-fiber function assessed with contact heat evoked potentials in humans. Pain 2007;132(1-2):102-107.

20. Wydenkeller S, Wirz R, Halder P. Spinothalamic tract conduction velocity estimated using contact heat evoked potentials: what needs to be considered. Clin Neurophysiol 2008;119(4):812-821.

21. Lauria G, Bakkers M, Schmitz C, Lombardi R, Penza P, Devigili G et al. Intraepidermal nerve fiber density at the distal leg: a worldwide normative reference study. J Peripher Nerv Syst 2010;15(3):202-207.

22. Reulen JP, Lansbergen MD, Verstraete E, Spaans F. Comparison of thermal threshold tests to assess small nerve fiber function: limits vs. levels. Clin Neurophysiol 2003;114(3):556-63. 
23. Yarnitsky D, Sprecher E, Zaslansky R, Hemli JA. Heat pain thresholds: normative data and repeatability. Pain 1995;60(3):329-32.

24. Yarnitsky D, Sprecher E. Thermal testing: normative data and repeatability for various test algorithms. J Neurol Sci 1994;125(1):39-45.

25. Bakkers M, Merkies IS, Lauria G, Devigili G, Penz P, Lombardi R et al. Intraepidermal nerve fiber density and its application in sarcoidosis. Neurology 2009;73(14):1142-1148.

26. Greffrath W, Baumgartner U, Treede RD. Peripheral and central components of habituation of heat pain perception and evoked potentials in humans. Pain 2007;132(3):301-311.

27. WarbrickT, Derbyshire SW, Bagshaw AP. Optimizing the measurement of contact heat evoked potentials. J Clin Neurophysiol 2009;26(2):117-122.

28. Hosmer DW, Lemeshow S. Applied Logistic Regression. New York: John Wiley \& Sons, inc., 2000.

29. DeLong ER, DeLong DM, Clarke-Pearson DL. Comparing the areas under two or more correlated receiver operating characteristic curves: a nonparametric approach. Biometrics 1988;44(3):837-845.

30. Granovsky Y, Granot M, Nir RR, Yarnitsky D. Objective correlate of subjective pain perception by contact heat-evoked potentials. J Pain 2008;9(1):53-63.

31. Harkins SW, Price DD, Roy A, Itskovich WV, Fei DY. Somatosensory evoked potentials associated with thermal activation of type II Adelta mechanoheat nociceptive afferents. Int J Neurosci 2000;104(1-4):93111.

32. Wang AL, Mouraux A, Liang M, lannetti GD. Stimulus novelty, and not neural refractoriness, explains the repetition suppression of laser-evoked potentials. J Neurophysiol;104(4):2116-24.

33. Agostinho CM, Scherens A, Richter H, Schaub C, Rolke R, Treede RD et al. Habituation and short-term repeatability of thermal testing in healthy human subjects and patients with chronic non-neuropathic pain. Eur J Pain 2009;13(8):779-785.

34. Treede RD. Peripheral acute pain mechanisms. Ann Med 1995;27(2):213-216.

35. Kramer JL, Taylor P, Haefeli J, et al. Test-retest reliability of contact heat-evoked potentials from cervical dermatomes. J Clin Neurophysiol 2012;29(1):70-75.

36. Wong MC, Chung JW. Feasibility of contact heat evoked potentials for detection of diabetic neuropathy. Muscle Nerve 2011;44(6):902-906.

37. Parson HK, Nguyen VT, Orciga MA, Boyd AL, Casellini CM, Vinik Al. Contact heat-evoked potential stimulation for the evaluation of small nerve fiber function. Diabetes Technol Ther 2013;15(2):150-157.

38. Itskovich WV, Fei DY, Harkins SW. Psychophysiological and psychophysical responses to experimental pain induced by two types of cutaneous thermal stimuli. Int J Neurosci 2000;105(1-4):63-75.

39. Kramer JL, Haefeli J, Curt A, Steeves JD. Increased baseline temperature improves the acquisition of contact heat evoked potentials after spinal cord injury. Clin Neurophysiol 2012;123(3):582-589.

40. Truini A, Galeotti F, Cruccu G, Garcia-Larrea L. Inhibition of cortical responses to Adelta inputs by a preceding C-related response: testing the "first come, first served" hypothesis of cortical laser evoked potentials. Pain 2007;131(3):341-347. 



\section{PART III}

\section{CLINICAL AND}

\section{CLINIMETRIC ASPECTS OF}

SMALL FIBER NEUROPATHY 

Chapter 5

\section{INCIDENCE AND \\ PREVALENCE OF}

SMALL FIBER NEUROPATHY:

A SURVEY IN THE

NETHERLANDS

${ }^{1}$ Department of Neurology, Maastricht University Medical Center, Maastricht, the Netherlands

2 Department of Neurology, Spaarne Hospital, Hoofddorp, the Netherlands

Neurology 2013;81:1-5. Wolters Kluwer Health Lippincott Williams \& Wilkins $\odot$ 


\section{ABSTRACT}

Objective: To determine the minimum incidence and minimum prevalence rates of small-fiber neuropathy (SFN) in a well-defined region in the southern part of the Netherlands.

Methods: In this cross-sectional study with retrospective data collection, we used data of patients diagnosed with pure SFN at our Small Fiber Neuropathy Center between January 2006 and December 2011 to calculate minimum incidence and prevalence rates.

Results: A total of 88 patients were diagnosed with SFN (mean age 56.9 years, SD 11.8, range 34- 81; 44.3\% women, 55.7\% men). The overall minimum incidence over 2010 and 2011 was 11.73 (95\% confidence interval 7.12-18.22) cases/100,000 inhabitants/ year. The overall minimum prevalence was 52.95 (95\% confidence interval 42.47-65.23) cases/100,000. Incidence and prevalence rates were higher in men than in women, as were the rates in elderly patients compared with younger patients.

Conclusions: The minimum incidence and prevalence rates of SFN are presented. We found that SFN is more frequently seen in men and more often diagnosed in elderly patients. These rates probably are an underestimation and are expected to increase in the coming years, since the awareness of SFN is increasing worldwide. 


\section{INTRODUCTION}

Small-fiber neuropathy (SFN) is characterized by neuropathic pain, sensory symptoms, and autonomic dysfunction.' Thinly myelinated Ad-fibers and unmyelinated C-fibers are selectively involved.'

SFN is diagnosed based on typical SFN-related symptoms, normal nerve conduction tests, reduced intraepidermal nerve fiber density (IENFD), and/or abnormal temperature threshold tests., 2 To assess autonomic function, the Thermoregulatory Sweat Test, Sympathetic Skin Responses, and Quantitative Sudomotor Axon Reflex Test have been used, but their definite role in diagnosing SFN needs to be established. . $^{3-5}$ SFN is increasingly recognized as a distinctive entity. The percentage of patients with idiopathic SFN, in which no underlying cause is identified, varies considerably from $24 \%$ to $93 \% .^{6-8}$ Recently, a substantial portion was found to carry novel mutations in the SCN9A gene. ${ }^{9}$

No conclusive data on incidence or prevalence rates of SFN have been published. Until recently, ${ }^{2,7}$ various diagnostic criteria and skin biopsy techniques were used, , 10, 11 and no comprehensive age-and sex-adjusted normative reference values for IENFD were available. ${ }^{12,13}$ Also, the frequency of SFN in various case series varied and was most likely biased by different selection criteria. ${ }^{2,7}$ Therefore, the aim of the current cross-sectional study with retrospective data collection was to determine the minimum incidence and minimum prevalence rates of SFN in a predefined region in the southern part of the Netherlands.

\section{METHODS}

The current study was performed at the Small Fiber Neuropathy Center of the Maastricht University Medical Center (MUMC). Since 2005, after establishing a skin biopsy laboratory, ${ }^{12}$ our center has been serving as a tertiary referral center for patients with (suspected) SFN. The MUMC has a unique position because it is the only hospital in Maastricht and surroundings (most southern part of the Netherlands) providing regional secondary care for patients and thus having an ideal geographical position for epidemiologic research. This region, the basic catchment area of the MUMC, is defined by post-code (zip-code) regions.

We collected data of all patients seen between January 2006 and December 2011 at our institute with (suspected) SFN. Subsequently, all patients living in the basic catchment area of our hospital were selected. For these patients, a validated symptoms inventory questionnaire, 12,14 and results of all additional tests were checked and only those with a definite diagnosis of pure SFN were eventually included in this study. SFN was diagnosed based on the presence of at least 2 of the following symptoms not otherwise explained: neuropathic pain (burning, shooting, prickling, or itching), sheet 
or sock intolerance, restless legs syndrome, autonomic dysfunction (sicca syndrome, accommodation problems, hyperhidrosis or hypohidrosis, micturition disturbances, impotence and/or diminished ejaculation or lubrication, bowel disturbances, hot flushes, orthostatic dizziness, cardiac palpitations) and clinical signs of small-fiber damage (e.g., pinprick loss, thermal sensory loss, allodynia, or hyperalgesia), normal nerve conduction study, and reduced IENFD at the ankle and/or abnormal quantitative sensory testing thermal thresholds at the foot.1,2,13

Worldwide normative values for age and sex for IENFD measurement with PGP9.5 staining are available for the distal leg. ${ }^{13}$ A reduction in IENFD below the fifth percentile is considered confirmatory for a diagnosis of SFN. ${ }^{5,13}$

For Temperature Threshold Testing, we used published normative values according to Yarnitsky. ${ }^{15}$ We assessed thresholds at the foot dorsum and the thenar eminence on both sides, using the method of levels and the method of limits. Heat pain was recorded with the method of limits and patients had to immediately react to pain sensation. ${ }^{4}$ Abnormality of a measurement was based on z-value statistics, and was considered abnormal when its z value exceeded $2.5 .^{4}$

We obtained population numbers for the basic catchment area of the MUMC by sex and age (in the age categories of 0-20, 20-65, and 65 years or older) from Statistics Netherlands, an organization responsible for official Dutch statistics and producing European (community) statistics. ${ }^{16}$ On January 1, 2011, there were 205,424 inhabitants in our catchment area, of which 166,203 were 20 years or older (80,350 male). These adults were considered the reference population at risk for the current study. Of these, 126,091 were 20-65 years (62,488 male), whereas 40,112 were 65 years or older (17,862 male). Using these population numbers from January 1, 2011, we calculated minimum incidence rates for the patients from our basic catchment area with newly diagnosed SFN in 2010 or 2011, and for minimum prevalence rates, we used the data of all patients from our basic catchment area diagnosed with SFN between January 2006 and December 2011. Standard protocol approvals, registrations, and patient consents. The MUMC's Medical Ethics Committee and Board of Directors approved this study. According to the Code of Conduct for the use of data in Health Research, ${ }^{17}$ for this type of retrospective study, informed consent does not need to be obtained if the data are used anonymously and patients are given the opportunity to object against the use of their medical and personal data for research (which is the case in the MUMC).

\section{RESULTS}

Between January 2006 and December 2011, a total of 508 patients have been examined at our SFN center for suspected SFN. Of these, 117 originated from the basic catchment area of our hospital. Twenty-nine patients did not meet the criteria for the diagnosis SFN, leaving 88 patients for this study. Among these 88 eligible patients (mean age 
56.9 years, SD 11.8, range 34-81), 49 were males (55.7\%; mean age 55.7 years, SD 12.4, range 34-81) and 39 females (44.3\%; mean age 58.3 years, SD 10.3, range 39-79). Most patients (98\%) were Caucasian. In 52\%, an underlying cause for SFN was identified (table 1).

Table 1. Causes of small fiber neuropathy in this cohort of 88 patients.

\begin{tabular}{cc}
\hline Causes & Number of patients (\%) \\
\hline Idiopathic & $42(47.7)$ \\
Sarcoidosis & $22(25.0)$ \\
Diabetes Mellitus & $6(6.8)$ \\
Drug related* & $5(5.7)$ \\
Vitamin B6 intoxication & $4(4.5)$ \\
Hyperthyroidism & $3(3.4)$ \\
MGUS & $2(2.3)$ \\
SCN9A mutation & $2(2.3)$ \\
Alcohol overuse & $1(1.1)$ \\
Hypertriglyceridemia & $1(1.1)$ \\
\hline
\end{tabular}

\section{Chapter}

* all were related to chemotherapeutic drugs

MGUS= monoclonal gammopathy of unknown significance

Upon neurologic consultation, nearly $8 \%$ of these 88 patients reported having symptoms for more than 10 years. Thirty-five percent of patients experienced burning pain, and autonomic symptoms were reported in $47.7 \%$ of the cases (table 2). Most frequently mentioned autonomic symptoms were sicca symptoms (dry eyes and/or dry mouth) (19.3\%), hyperhidrosis (18.2\%), and defecation disturbances (13.6\%).

Table 2. Reported autonomic symptoms in this cohort of 88 patients.

\begin{tabular}{cc}
\hline Autonomic symptoms & Number of patients (\%) \\
\hline Sicca symptoms (dry eyes and/or dry mouth) & $17(19.3)$ \\
Hyperhidrosis & $16(18.2)$ \\
Defecation disturbances & $12(13.6)$ \\
Orthostatic dizziness & $10(11.4)$ \\
Micturation disturbances & $9(10.2)$ \\
Hot flushes & $5(5.7)$ \\
Sexual dysfunction & $5(5.7)$ \\
Gastroparesis & $2(2.3)$ \\
Accomodation problems & $2(2.3)$ \\
Hypohidrosis & $1(1.1)$ \\
Cardiac palpitations & $1(1.1)$ \\
\hline
\end{tabular}

In 2010 and 2011, 216 patients were examined; 57 of these patients lived in our basic catchment area of which 39 were diagnosed with definite SFN. Among these 39 patients (mean age 57.5 years, SD 12.3, range 38-81), there were 25 males (64.1\%; mean age 57.8 years, SD 12.6, range 38-81) and 14 females (35.9\%; mean age 56.8 years, SD 12.1, range 39-79). The overall minimum incidence over 2010 and 2011 was 11.73 (95\% confidence interval 7.12-18.22) cases/ 100,000 inhabitants/year. The overall 
minimum prevalence was 52.95 (95\% confidence interval 42.47-65.23) cases/100,000 inhabitants (see table 3 for additional data on incidence and prevalence).

Table 3. Minimum incidence and minimum prevalence rates of small fiber neuropathy.

\begin{tabular}{lccc}
\hline & All adults & Males & Females \\
\hline Incidence: cases/1000.000/y (95\% Cl) & & & \\
Overall ( $\mathrm{n}=39 ; 25$ males, 14 females) & $11.73(7.12-18.22)$ & $15.56(8.17-26.88)$ & $8.15(3.28-16.80)$ \\
20-65 year old ( $\mathrm{n}=28 ; 18$ males, 10 females) & $11.10(6.07-18.63)$ & $14.40(6.59-27.34)$ & $7.86(2.55-18.34)$ \\
65 year or older ( $\mathrm{n}=11 ; 7$ males, 4 females) & $13.71(4.77-30.82)$ & $19.59(4.78-53.21)$ & $8.99(1.09-32.47)$ \\
Prevalence: cases/100.000 (95\% Cl) & & & \\
Overall ( $\mathrm{n}=88 ; 49$ males, 39 females) & $52.95(42.47-65.23)$ & $60.98(45.12-80.62)$ & $45.43(32.30-62.09)$ \\
20-65 year old $(\mathrm{n}=65 ; 38$ males, 27 females) & $51.55(39.79-65.70)$ & $60.81(43.04-83.46)$ & $42.45(27.98-61.76)$ \\
65 year or older $(\mathrm{n}=23 ; 11$ males, 12 females) & $57.34(36.35-86.02)$ & $61.58(30.75-110.16)$ & $53.93(27.87-94.19)$ \\
\hline Cl= confidence interval & & &
\end{tabular}

\section{DISCUSSION}

The current study presents estimated minimum incidence and minimum prevalence rates for the diagnosis of SFN. As shown in table 3, minimum incidence and prevalence rates showed a trend toward higher values in men and in patients aged 65 years and older. The general annual incidence of 11.73 cases/ 100,000 for SFN is significantly higher than the reported incidence rates in inflammatory large-fiber neuropathies, such as Guillain-Barré syndrome (GBS) and chronic inflammatory demyelinating polyneuropathy (CIDP). For example, the reported incidence of GBS in Western countries ranges from 0.89 to 1.89 cases (median 1.11)/100,000 person-years. ${ }^{18}$ For CIDP, incidence rates between 1 in 100,000 in southeast United Kingdom and 7.7 in 100,000 in northern Norway have been reported..$^{19}$ Because the impact of SFN on quality of life can be as devastating as seen in patients with large-fiber neuropathies, its recognition is equally important. SFN has been receiving increasing attention in the last 15 years, since the introduction of IENFD measurements. ${ }^{20}$ The number of patients diagnosed with this general painful neuropathy is increasing rapidly because of better recognition of the disease. ${ }^{2,7,21,22}$ This in part explains the number of patients with a long-standing history of symptoms before diagnosis. Where prevalence rates are not influenced by this fact, incidence rates are. We therefore defined an incident case as being newly diagnosed at our SFN center in 2010 or 2011. Our findings represent minimum incidence and minimum prevalence rates because by far not every patient with SFN will have been recognized and referred to our SFN center. However, we believe that we have estimated the best possible reliable rates by restricting this study to the population of the basic catchment area of our center for which we are the only hospital and therefore also provide regional secondary care. In addition, this population is known to be stable because few people move in and out of this area and because of the nonlethal nature of SFN. 
There are some methodological issues that should be addressed. First, in our study population, sarcoidosis seems to be a common cause of SFN. The recognition of the relation between sarcoidosis and $\mathrm{SFN}^{12,23}$ has led to an increased awareness, particularly at our center, serving as a tertiary referral center for both sarcoidosis and SFN. Therefore, a selection bias of sarcoidosis as an underlying cause of SFN is conceivable. In our diagnostic workup for SFN, tests for sarcoidosis are included. However, most patients with sarcoidosis were already diagnosed with this disease before being evaluated for suspected SFN. As all other patients, they also underwent testing for other underlying causes of SFN. Sarcoidosis was considered the most likely cause of SFN if no other causes were found. The prevalence of sarcoidosis is known to be higher among African Americans and northern European Caucasians (especially Scandinavians). Because approximately $98 \%$ of our SFN patients and $99 \%$ of the sarcoidosis patients were western European Caucasian, we believe this did not contribute to the bias toward sarcoidosis. However, interpretation of these points should be done with great caution because the primary aim of the current study was not directed toward the etiology of SFN. Second, the "idiopathic" group is considered a potential limitation because (yet undetermined) hereditary causes should be strongly considered. We did not routinely test for all known hereditary sensory and autonomic neuropathies (because of cost limitations). However, many of these neuropathies have specific clinical features generally not compatible with the pure SFN clinical spectrum. ${ }^{24,25}$ In addition, the "idiopathic" group may also contain patients unrecognized as having autoimmune-mediated neuropathies such as Sjögren's syndrome or neuropathy associated with sicca complex, as many of our patients mentioned sicca symptoms. We routinely performed serology testing (SS-A, SS-B, antinuclear antibodies) as part of the diagnostic workup in all of our patients. If systemic disease was suspected, patients subsequently underwent Schirmer test and lip biopsy. A negative serology does not rule out these conditions, but makes it less likely, especially when combined with a negative Schirmer test and lip biopsy. Under these criteria, none of our patients were diagnosed with Sjögren's syndrome. However, it is still possible that an inflammatory condition (including Sjögren's syndrome) could be an underlying cause. Also, "dry eyes" might actually be corneal hyperalgesia; sensory neuropathy involving the corneal nociceptive system may lead to neuropathic pain, felt and reported as dry eyes. ${ }^{26}$

Despite these limitations, the current study presents minimum incidence and minimum prevalence rates of SFN in a well-defined region in the southern part of the Netherlands. These rates are probably an underestimation and are expected to increase in the coming years, since the awareness of SFN is increasing worldwide. 


\section{REFERENCES}

1. Hoeijmakers JG, Faber CG, Lauria G, Merkies IS, Waxman SG. Small-fibre neuropathies-advances in diagnosis, pathophysiology and management. Nat Rev Neurol 2012;8:369-379.

2. Tesfaye S, Boulton AJ, Dyck PJ, et al. Diabetic neuropathies: update on definitions, diagnostic criteria, estimation of severity, and treatments. Diabetes Care 2010;33:2285-2293.

3. Low VA, Sandroni P, Fealey RD, Low PA. Detection of small-fiber neuropathy by sudomotor testing Muscle Nerve 2006;34:57-61.

4. Hoitsma $E$, Drent M, Verstraete $E$, et al. Abnormal warm and cold sensation thresholds suggestive of small-fibre neuropathy in sarcoidosis. Clin Neurophysiol 2003;114:2326-2333.

5. Lauria G, Hsieh ST, Johansson O, et al. European Federation of Neurological Societies/Peripheral Nerve Society Guideline on the use of skin biopsy in the diagnosis of small fiber neuropathy. Report of a joint task force of the European Federation of Neurological Societies and the Peripheral Nerve Society. Eur $J$ Neurol 2010;17:903-912, e944-909.

6. Lacomis D. Small-fiber neuropathy. Muscle Nerve 2002;26:173-188.

7. Devigili G, Tugnoli V, Penza P, et al. The diagnostic criteria for small fibre neuropathy: from symptoms to neuropathology. Brain 2008;131:1912-1925.

8. Bednarik J, Vlckova-Moravcova E, Bursova S, Belobradkova J, Dusek L, Sommer C. Etiology of small-fiber neuropathy. J Peripher Nerv Syst 2009;14:177-183.

9. Faber CG, Hoeijmakers JG, Ahn HS, et al. Gain of function Nanu1.7 mutations in idiopathic small fiber neuropathy. Ann Neurol 2012;71:26-39.

10. Sumner CJ, Sheth S, Griffin JW, Cornblath DR, Polydefkis M. The spectrum of neuropathy in diabetes and impaired glucose tolerance. Neurology 2003;60:108-111.

11. Vlckova-Moravcova E, Bednarik J, Dusek L, Toyka KV, Sommer C. Diagnostic validity of epidermal nerve fiber densities in painful sensory neuropathies. Muscle Nerve 2008;37:50-60.

12. Bakkers M, Merkies IS, Lauria G, et al. Intraepidermal nerve fiber density and its application in sarcoidosis. Neurology 2009;73:1142-1148.

13. Lauria G, Bakkers M, Schmitz C, et al. Intraepidermal nerve fiber density at the distal leg: a worldwide normative reference study. J Peripher Nerv Syst 2010;15:202-207.

14. Bakkers M, Faber CG, Drent $M$, et al. Pain and autonomic dysfunction in patients with sarcoidosis and small fibre neuropathy. J Neurol 2010;257:2086-2090.

15. Yarnitsky D, Sprecher E. Thermal testing: normative data and repeatability for various test algorithms. J Neurol Sci 1994;125:39-45.

16. Statistics Netherlands [online]. Available at: <http://www.cbs.nl/en-GB/menu/home/default.htm>. Accessed February 13

17. Federa [online]. Available at: www.federa.org/codes-conduct. Accessed February 13.

18. Yuki N, Hartung HP. Guillain-Barre syndrome. N Engl J Med 2012;366:2294-2304.

19. Vallat JM, Sommer C, Magy L. Chronic inflammatory demyelinating polyradiculoneuropathy: diagnostic and therapeutic challenges for a treatable condition. Lancet neurology 2010;9:402-412.

20. McCarthy BG, Hsieh ST, Stocks A, et al. Cutaneous innervation in sensory neuropathies: evaluation by skin biopsy. Neurology 1995;45:1848-1855

21. Sorensen L, Molyneaux L, Yue DK. The relationship among pain, sensory loss, and small nerve fibers in diabetes. Diabetes Care 2006;29:883-887.

22. Walk D, Wendelschafer-Crabb G, Davey C, Kennedy WR. Concordance between epidermal nerve fiber density and sensory examination in patients with symptoms of idiopathic small fiber neuropathy. J Neurol Sci 2007;255:23-26.

23. Hoitsma E, Marziniak M, Faber CG, et al. Small fibre neuropathy in sarcoidosis. Lancet 2002;359:20852086.

24. Houlden H, Blake J, Reilly MM. Hereditary sensory neuropathies. Curr Opin Neurol 2004;17:569-577.

25. Pareyson D. Diagnosis of hereditary neuropathies in adult patients. J Neurol 2003;250:148-160.

26. Rosenthal P, Borsook D. The corneal pain system. Part I: the missing piece of the dry eye puzzle. Ocul Surf 2012;10:2-14. 
Chapter

5 

Chapter 6

\section{PAIN AND AUTONOMIC}

DYSFUNCTION IN PATIENTS

WITH SARCOIDOSIS AND

SMALL FIBER NEUROPATHY

M. Bakkers ${ }^{1 *}$, C.G. Faber ', M. Drent ${ }^{2}$, M.C.E. Hermans ', S.I. van Nes ${ }^{3}$, G. Lauria ${ }^{4}$, M. De Baets ${ }^{5}$, I.S.J Merkies ${ }^{1,3,6}$

'Department of Neurology, Maastricht university medical centre, Maastricht, the Netherlands

${ }^{2}$ Department of Respiratory Medicine, Interstitial Lung Disease care team, Maastricht University

Medical Centre, P.O. Maastricht, the Netherlands

${ }^{3}$ Department of Neurology, Erasmus Medical Centre Rotterdam, Rotterdam, the Netherlands

${ }^{4}$ Neuromuscular diseases unit, IRCCS Foundation, National Neurological Institute

"Carlo Besta", Milan, Italy

${ }^{5}$ Department of Neuroscience, School for Mental Health and Neuroscience,

Maastricht, the Netherlands

${ }^{6}$ Department of Neurology, Spaarne Hospital, Hoofddorp, the Netherlands 


\section{ABSTRACT}

Small fiber neuropathy (SFN) has been demonstrated in sarcoidosis. However, a systematic analysis of neuropathic pain and autonomic symptoms, key features of SFN, has not been performed. Clinimetric evaluation of pain and autonomic symptoms using the neuropathic pain scale (NPS) and the modified Composite Autonomic Symptoms Scale (mCOMPASS) was used in sarcoidosis patients for this study. A total of 91 sarcoidosis patients ( $n=23$ without SFN symptoms, $n=43$ with SFN symptoms but normal intraepidermal nerve fiber density (IENFD), $n=25$ with SFN symptoms and reduced IENFD) were examined. NPS and mCOMPASS were assessed twice (reliability studies). Severity of pain was compared between the subgroups. Correlation between NPS and a visual analogue pain scale (VAS) was assessed (validity studies). Healthy controls $(n=105)$ completed the mCOMPASS for comparison with patients' scores. Patients with sarcoidosis, SFN complaints, and reduced IENFD demonstrated more severe pain scores on the NPS. The mCOMPASS differentiated between subjects with and without SFN symptoms. A significant correlation was obtained between the NPS and VAS, indicating good construct validity. Good reliability values were obtained for all scales. The use of the NPS to evaluate SFN symptoms is suggested, as it shows differences between patients with SFN symptoms with normal or reduced IENFD values. The mCOMPASS might be used to select patients for further testing. 


\section{INTRODUCTION}

Small fiber neuropathy (SFN) is a disorder with selective involvement of small-caliber myelinated ( $\mathrm{Ad}$ ) and unmyelinated (C) nerve fibers, characterized by neuropathic pain and autonomic symptoms. Diagnosis is usually made on the basis of clinical features (neuropathic pain, loss of pinprick and temperature sensation), in combination with abnormal specialized tests such as the assessment of intraepidermal nerve fiber density (IENFD) in skin biopsy, temperature sensation tests for sensory fibers, and sudomotor and cardiovagal testing for autonomic fibers. ${ }^{1}$

Pain is a frequent complaint in sarcoidosis, an inflammatory multisystem disorder of unknown etiology which may involve any part of the nervous system, ${ }^{2}$ and pain is related to lower quality of life. ${ }^{3}$ We recently demonstrated SFN to be frequent in sarcoidosis. ${ }^{4}$ However, neither the type or intensity of pain, nor the extent to which autonomic symptoms may occur in SFN in sarcoidosis have been studied systematically in this condition. The current study describes the various aspects of pain and autonomic symptoms in patients with sarcoidosis using the neuropathic pain scale (NPS) and a modified Composite Autonomic Symptoms Scale (mCOMPASS), and assesses validity and reliability of these outcome measures.

Chapter

\section{PARTICIPANTS AND METHODS}

\section{Participants}

One hundred and five healthy volunteers were recruited from hospital personnel, relatives and friends of patients, at sports accommodations, and informal meetings for the elderly. Inclusion criteria were: no pain or other neurological complaints, no history of alcohol abuse, no diseases that may cause sensory deficit or pain sensation, and normal findings at neurological examination. ${ }^{5}$

Patients referred to the Maastricht Sarcoidosis Management Centre, a referral center for sarcoidosis in The Netherlands, were screened for eligibility. We included 91 patients in the study. Inclusion criteria were: diagnosis of sarcoidosis, ${ }^{6}$ lucid consciousness, no alcohol abuse, no usage of immunosuppressant drugs in the past 6 months, no diseases that may cause sensory deficit, and no signs of central nervous system involvement or large fiber neuropathy (no abnormal nerve conduction studies). After inclusion, patients were categorized as either "no SFN", or, when having SFN symptoms as "possible SFN". A patient was classified as having SFN symptoms when he or she reported at least one of the following symptoms, not otherwise explained: burning pain in extremities, dry mouth or eyes, changes in sweating, flushes, gastrointestinal dysfunction (constipation, diarrhea), cardiac complaints (palpitation, dizziness at standing up), urogenital dysfunction (sexual dysfunction, incontinence). ${ }^{5}$ 


\section{Scale selection}

The NPS was designed to assess distinct pain qualities associated with neuropathic pain and has been used in peripheral neurological conditions. ${ }^{7}$ The questionnaire rates ten different aspects of pain on a numerical 0-10 scale. Addressed are intensity and unpleasantness of pain in general; intensity of sharpness, hotness, dullness, coldness, skin sensitivity, itching; and intensity of surface and deep pain.

The horizontal visual analogue pain scale (VAS) is a $10 \mathrm{~cm}$ horizontal line, depicting no pain at the left and worst pain ever at the right side. Patients mark their pain intensity at the line. It is considered to reliably assess a patient's experience. ${ }^{8}$ The original Composite Autonomic Symptom Scale (COMPASS), validated in patients with autonomic failure and non-autonomic neuropathies, correlates well with autonomic function tests. ${ }^{9}$ Its 73 questions concern different aspects of the autonomic system: orthostatic intolerance and reflex syncope, secretomotor, vasomotor, pupillomotor, urogenital, gastrointestinal, and sleep difficulties.

\section{Translation and modification}

The translation of the NPS and mCOMPASS was performed according to the international guidelines [10]. ${ }^{10}$ Some domains of the COMPASS were simplified by a clinimetrician (ISJM), and questions on female sexuality were added, reflecting an equivalent score for male sexuality, resulting in a 65 item modified scale (mCOM-PASS), with a sum score of 200 for both men and women.

\section{Skin biopsies}

All participants underwent skin biopsy for intraepidermal nerve fiber density (IENFD) determination according to European guidelines. ${ }^{11}$ Biopsies were taken $10 \mathrm{~cm}$ above the lateral malleolus. Normative values were used to determine normal versus impaired IENFD findings. ${ }^{5}$ Patients were further divided into subgroups according to the presence of symptoms combined with skin biopsy results: group $A$, patients without SFN symptoms; B, patients with SFN symptoms but normal IENFD; and C, patients with SFN symptoms and reduced IENFD. We expected those with both symptoms and reduced IENFD to be most affected, IENFD being considered an objective tool to diagnose SFN. ${ }^{11,12}$

\section{Study design and statistical analysis}

The study was approved by the medical ethical committee of the Maastricht University Medical Centre (Central Committee for Human Related Research, identifier number p06.0066L/MEC 05-224), in accordance with the ethical standards laid down in the 1964 Declaration of Helsinki. Informed consent was obtained from all participants prior to the study. All patients were examined at the Maastricht University Medical Centre. Examination took take place in a comfortable, temperature-controlled room. Questionnaires were provided with written instructions. Healthy controls were requested to 
complete the mCOMPASS. Data collection, entry, and management were performed using the Teleform automated processing system.

\section{Reliability and validity studies}

Patients completed the NPS and mCOMPASS twice, within 2-4 weeks, without having access to their previous answers (test-retest reliability; weighted kappa-statistic ( $\mathrm{K}$ ) measures). ${ }^{13}$ We examined the discriminatory validity of the NPS in the various patients' subgroups in relation to severe pain, defined as a numerical rating score $>5$ on a NPS question (Chi-square test). Correlation studies between the NPS (item pain intensity) and VAS pain scales were also performed (convergent validity of NPS; Spearman's Rank tests). For the mCOMPASS domains, subgroups comparison (one-way ANOVA + Bonferonni corrections) was performed. All analyses were performed using Stata 10.0 for Windows XP.

\section{RESULTS}

From October 2006 to July 2008, 105 healthy controls (54 women and 51 men; mean age 45.1 (SD: 14.6; range: 20-79) years), and 91 sarcoidosis patients (41 women, 50 men; age 45.5 (SD: 9.1; range: 27-70) years) were included in the study. The patients with sarcoidosis were subdivided into three groups: Group A, patients without SFN symptoms ( $n=23$ ); B, patients with SFN symptoms but normal IENFD ( $n=43)$; and C, patients with SFN symptoms and impaired IENFD $(n=25)$. In group A, 35\% of patients reported severe pain, in group B 65\%, in group C $76 \%$. Not only intensity of pain, but also various other qualities of pain were significantly more severe in group C compared to the other subgroups: "sharpness", "burning", "dullness", "unpleasant pain sensation", and "intense surface pain" (Figure 1). More severe pain was also seen for the qualities "sensitivity of skin" and "intense deep pain" sensations, but the differences did not reach significance.

Good correlation was obtained between the NPS and VAS pain scales (NPS (intensity) versus VAS: Spearman's rho 0.73).

The mCOMPASS demonstrated higher scores (more autonomic dysfunction related symptoms) for all domains and sum scores in the patients with SFN complaints (groups B and C) compared to group A and healthy controls, but did not discriminate between the groups B and C (Figure 2). For the sexual disorders and syncope domains, no discrimination between the subgroups and healthy controls was obtained.

Reliability studies showed good test-retest scores for all domains of the NPS and mCOMPASS (weighted kappa 0.60-0.95), except for the mCOMPASS syncope domain (0.39). The mCOMPASS sum score had a kappa of 0.88. 
Figure 1. Graphical representation of several items of the neuropathic pain scale (NPS) in patients with sarcoidosis.

No pain sensation (score $=0$ )

Minor to moderate pain sensation (score $=1-4$ )

Severe pain sensation (score $\geq 5$ )
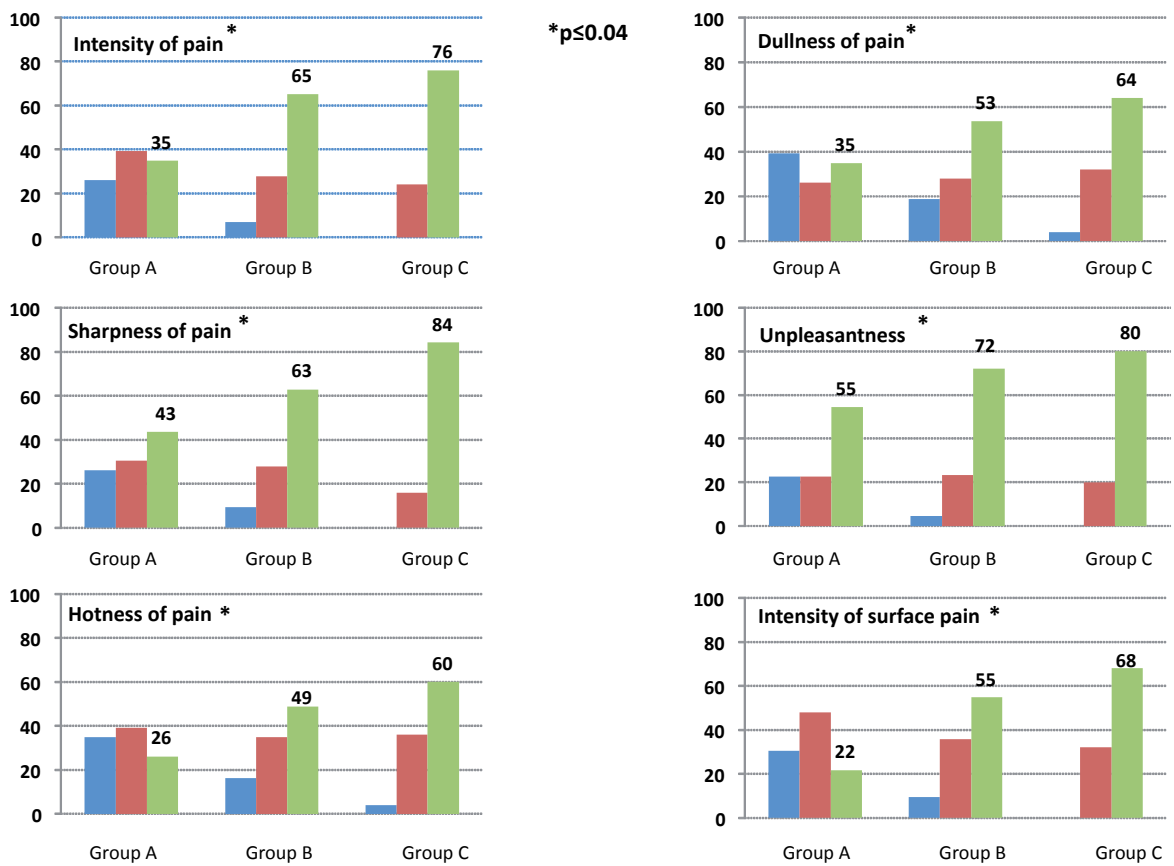

Those pain qualities with significant differences between subgroups are shown $(p \leq 0.04)$. Bars show percentage of patients in the three patient groups with (in blue) no, (red) minor to moderate, or (green) severe pain. Group A ( $n=23)$, sarcoidosis patients without small fiber neuropathy (SFN) symptoms. Group B $(n=43)$, sarcoidosis patients with SFN symptoms but normal intrapeidermal nerve fiber density (IENFD).

Group C $(n=25)$, sarcoidosis patients with SFN symptoms and reduced IENFD.

Figure 2. Box plots showing median and interquartile ranges of the modified version of the Composite Autonomic Symptoms Scale (mCOMPASS) for healthy controls and various subgroups of patients with sarcoidosis.

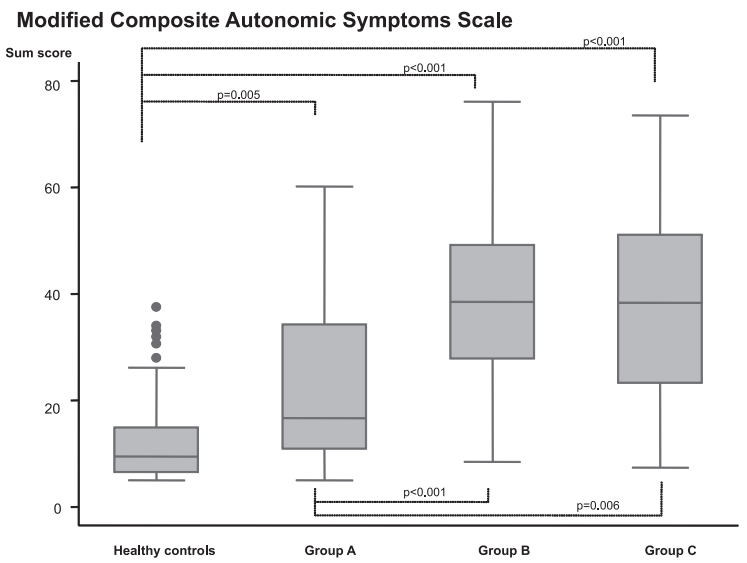

Comparison of subgroups by one way Anova+Bonferonni corrrections.

Group A ( $n=23)$, patients without small fiber neuropathy (SFN) symptoms. Group B $(n=43)$, patients with SFN symptoms but normal intrapeidermal nerve fiber density (IENFD).

Group C $(n=25)$, patients with SFN symptoms and reduced IENFD. Between groups $B$ and $C$, differences were not significant. 


\section{DISCUSSION}

The current study describes the various aspects of pain and autonomic dysfunction in patients with sarcoidosis using the NPS and the mCOMPASS. Various neuropathic pain modalities measured with the NPS were more severe in patients with SFN complaints and even more so in those with reduced IENFD values (group C). This tool may be of use in interventional studies; for example, in evaluating the efficacy of diseasemodifying therapies. ${ }^{14}$ As expected, higher mCOMPASS scores were found in patients with SFN symptoms. Nevertheless, no significant difference in sum scores was seen between group $B$ and $C$. Autonomic nerve fibers are of a different type compared to the measured ones in skin, and agreement between IENFD and autonomic deficits varies. ${ }^{15}$ Autonomic tests can be very time consuming though, with a high burden to patients. ${ }^{16}$ Therefore, the use of this scale is suggested to select patients in whom further testing for SFN might be warranted.

The NPS correlated well with the VAS, indicating good construct validity. Both the NPS and the mCOMPASS demonstrated good test-retest reliability.

There are some methodological issues that should be addressed. Some caution is requested when interpreting the results, since the sample sizes of the various subgroups were relatively small. Also, we did not exclude patients with painful sarcoidosis related problems such as arthritis. It may have been difficult for patients to differentiate between their pain origins, and it might explain why such a large proportion of patients in group A reports severe pain. However, the qualities regarded as typically neuropathic (sharp, hot, superficial pain) were especially more severe in patients with SFN symptoms and reduced IENFD. Furthermore, the mCOMPASS is a composite ordinal scale based on the classic test theory, which may have disadvantages. ${ }^{17}$ In conclusion, the neuropathic pain scale shows differences between patients with SFN symptoms with normal or reduced IENFD values, and its use is therefore suggested in future (interventional) studies of this condition. The presence of autonomic symptoms as measured by the mCOMPASS might be used to select patients for further testing with regard to possible SFN. 


\section{REFERENCES}

1. Hoitsma E, Reulen JP, de Baets M, Drent M, Spaans F, Faber CG. Small fiber neuropathy: a common and important clinical disorder. J Neurol Sci 2004;227:119-130.

2. Iannuzzi MC, Rybicki BA, Teirstein AS. Sarcoidosis. N Engl J Med 2007;357:2153-2165.

3. Hoitsma E, De Vries J, van Santen-Hoeufft M, Faber CG, Drent M. Impact of pain in a Dutch sarcoidosis patient population. Sarcoidosis Vasc Diffuse Lung Dis 2003;20:33-39.

4. Hoitsma E, Marziniak M, Faber CG, et al. Small fibre neuropathy in sarcoidosis. Lancet 2002;359:20852086.

5. Bakkers M, Merkies IS, Lauria G, et al. Intraepidermal nerve fiber density and its application in sarcoidosis. Neurology 2009;73:1142-1148.

6. Hunninghake GW, Costabel U, Ando M, et al. ATS/ERS/WASOG statement on sarcoidosis. American Thoracic Society/European Respiratory Society/World Association of Sarcoidosis and other Granulomatous Disorders. Sarcoidosis Vasc Diffuse Lung Dis 1999;16:149-173.

7. Galer BS, Jensen MP. Development and preliminary validation of a pain measure specific to neuropathic pain: the Neuropathic Pain Scale. Neurology 1997;48:332-338.

8. Ohnhaus EE, Adler R. Methodological problems in the measurement of pain: a comparison between the verbal rating scale and the visual analogue scale. Pain 1975;1:379-384.

9. Suarez GA, Opfer-Gehrking TL, Offord KP, Atkinson EJ, O'Brien PC, Low PA. The Autonomic Symptom Profile: a new instrument to assess autonomic symptoms. Neurology 1999;52:523-528.

10. Streiner D.L. GRN. Health measurement scales. A practical guide to their development and use, second ed. New York: Oxford University Press, 1998.

11. Lauria G, Cornblath DR, Johansson O, et al. EFNS guidelines on the use of skin biopsy in the diagnosis of peripheral neuropathy. Eur J Neurol 2005;12:747-758.

12. Lauria G, Lombardi R. Skin biopsy: a new tool for diagnosing peripheral neuropathy. Bmj 2007;334:11591162.

13. Cohen J. Weighted kappa: Nominal scale agreement with provision for scal disagreement or part credit. Psychol Bull 1968;70:213-230.

14. Baughman RP, Lower EE, du Bois RM. Sarcoidosis. Lancet 2003;361:1111-1118.

15. Singer W, Spies JM, McArthur J, et al. Prospective evaluation of somatic and autonomic small fibers in selected autonomic neuropathies. Neurology 2004;62:612-618.

16. Ravits JM. AAEM minimonograph \#48: autonomic nervous system testing. Muscle Nerve 1997;20:919937.

17. DeVellis RF. Classical test theory. Med Care 2006;44:S50-59. 
Chapter 6 

Chapter 7

\section{SMALL FIBERS, \\ LARGE IMPACT: QUALITY \\ OF LIFE IN SMALL \\ FIBER NEUROPATHY}

M. Bakkers', C. G. Faber', J.G.J. Hoeijmakers', G. Lauria MD², I.S.J. Merkies³

'Department of Neurology, Maastricht University Medical Centre, Maastricht, the Netherlands

${ }^{2}$ Neuromuscular diseases unit, IRCCS Foundation, National Neurological Institute

"Carlo Besta", Milan, Italy

${ }^{3}$ Department of Neurology, Spaarne Hospital, Hoofddorp, the Netherlands

Muscle \& Nerve 2014, 49(3):329-336. Wiley @ 


\section{ABSTRACT}

Introduction: The impact of small-fiber neuropathy (SFN) on patients' quality of life (QOL) has not been studied extensively. Our aim was to determine the impact of SFN on QOL and examine possible determinants. Methods: We examined a total of 265 patients diagnosed with SFN. The SFN Symptoms Inventory Questionnaire (SFN-SIQ), the pain Visual Analog Scale (VAS), and the generic SF-36 Health Survey were assessed. Regression studies were undertaken to evaluate determinants of functioning. Results: SFN patients demonstrated a severe overall reduction in QOL. The biggest deficits were in Role Functioning-Physical, Body Pain, and Physical Component Summary (PCS) scores. VAS scores, changed sweating pattern, dry mouth, and age were the strongest predictors for PCS, explaining 32\% of the QOL decrease. Conclusions: SFN leads to a reduction in overall QOL. The presence of pain and some autonomic symptoms explained only a small portion of the findings. 


\section{INTRODUCTION}

Small-fiber neuropathy (SFN), a disorder of thinly myelinated A-d and unmyelinated C fibers, is characterized by chronic and severe complaints, such as neuropathic pain and autonomic symptoms. SFN is associated with various disorders, such as metabolic (diabetes mellitus), infectious (human immunodeficiency virus), inflammatory (Sjögren's syndrome), and genetic (Fabry disease) diseases, but the cause is often idiopathic. The incidence and prevalence of SFN is unknown, ${ }^{1,2}$ but it is probably not rare. In patients with diabetes mellitus, for example, a disease with increasing incidence and prevalence, ${ }^{3}$ an estimated $16-20 \%$ have painful neuropathy., ${ }^{4,5}$ This has been largely attributed to small-fiber involvement. ${ }^{6}$

The condition and its diagnosis has been gaining interest in the last 15 years, since the introduction of quantification of intraepidermal nerve fiber density (IENFD) in skin biopsies. ${ }^{7}$ Although some have suggested that a reduced IENFD is a mandatory criterion for diagnosis of SFN, 89 various studies have proposed different definitions., 10-14 To date, the diagnosis of SFN relies on clinical features (neuropathic pain and autonomic symptoms not otherwise explained; loss of pinprick and temperature sensation without signs of large-fiber dysfunction) combined with abnormal quantification of IENFD and/or deficit in temperature threshold testing(TTT). $7,13,15-17$

Skin biopsy findings are considered the strongest contributors to the diagnosis of SFN, because higher diagnostic accuracy has been demonstrated when compared with clinical features and quantitative sensory testing (QST) results. ${ }^{18,19}$

The impact of SFN on quality of life (QOL) and its possible explicatory determinants have not been examined systematically in patients with SFN. The primary aims of this study were to determine QOL in a cohort of patients with SFN using the Medical Outcomes Study 36-item Short Form Health Status (SF-36) and to compare the results with reported normative data for the healthy Dutch population. ${ }^{20-23} \mathrm{Also}$, in this study we aimed to determine which SFN-related complaints would explain QOL findings through regression studies in this condition.

\section{METHODS}

\section{Patients and Eligibility}

The Maastricht University Medical Centre (MUMC) has experience in the diagnosis and management of patients with SFN. The MUMC serves as a tertiary referral center for patients with possible SFN, offering standardized evaluation. Between January 2009 and August 2011, all patients with a definite clinical diagnosis of SFN were approached for participation in this study. ${ }^{15}$ Eligibility was based on the following criteria: age 18 years and older; definite clinical diagnosis of SFN based on: (1) the presence of at least 2 of the following complaints not otherwise explained: burning feet, redness of 
the skin, dry eyes or mouth, orthostatic dizziness, bowel disturbances (constipation, diarrhea, irritability, gastroparesis, cramps), micturation disturbances, sweat changes (hyper/hypohidrosis), accommodation problems, impotence, diminished ejaculation or lubrication, flushes, and palpitations; (2) reduced IENFD when compared with ageand gender-matched normative values ${ }^{24}$ and/or (3) abnormal temperature threshold testing (TTT) when compared with normative values, ${ }^{25,26}$ (4) absence of large nerve fiber involvement on neurological examination (e.g., weakness, vibration threshold abnormalities as determined by Rydel- Seiffer graduated tuning fork $)^{27}$ and nerve conduction studies (examining at least median, fibular, tibial, and sural nerves, including late responses); and (5) possible presence of hyperpathia, diminished pain, and temperature sensation on examination. IENFD and TTT examinations were performed as previously reported ${ }^{28,29}$ and in accordance with the available guidelines. ${ }^{7}$ ${ }^{30}$ Briefly, a 3-mm punch biopsy was taken $10 \mathrm{~cm}$ above the lateral malleolus, and the number of individual nerve fibers crossing the dermal-epidermal junction were counted in 3 randomly taken sections $(50 \mathrm{~lm})$ after immunostaining with polyclonal rabbit anti-protein gene product 9.5 antibody (PGP-9.5; Ultraclone; Wellow, Isle-ofWight, UK). The linear density of IENF was calculated (IENF/mm of epidermal length, 1 of 2 observers, interobserver values 0.9). Findings were compared with normative data. ${ }^{24}$ TTTs for warm, cool, and heat pain modalities were assessed (TSA2001; Medoc, Ramat-Yishai, Israel) on the dorsum of both feet and thenar eminences. Results were considered abnormal if both method-of-limits and method-of-levels values were outside normative references. . $^{2,29,31}$

\section{Medical Ethics Approval}

The study was performed in accordance with ethics standards established by the 1964 Declaration of Helsinki and its later amendments. The investigation was approved by the medical ethics committee of the MUMC. The intentions of the study were explained to all patients, and informed consent was obtained from all patients before their inclusion in the study.

\section{Additional Investigations}

Additional investigations were performed to determine possible etiology of SFN. This included a complete history (including the use of alcohol, vitamins, or neurotoxic drugs), chest X-ray, and blood tests [blood cell counts, electrolytes, liver enzymes, creatinine, urea, lipids, fasting blood glucose, thyroid-stimulating hormone (TSH), free $T 4$, vitamin B6, serum immunofixation, autoantibodies for antinuclear antibodies (ANA), anti-neutrophil cytoplasmic antibodies (ANCA), SS-A/SS-B, and anti-sulfatide]; in selected patients, we also screened for Fabry disease (alfagalactosidase, in women combined with sequencing of the GLA gene) and tested for infection with human immunodeficiency virus (HIV) and Borrelia burgdorferi.

Assessment Scales. The Dutch V1 version of the SF-36 was used to assess aspects 
of QOL. This generic scale consists of 36 items assigned to the domains of Physical Functioning (10 items), Role Functioning-Physical (4), Role Functioning-Emotional (3), Social Functioning (2), Body Pain (2), Mental Health (5), Vitality (4), General Health Perception (5), and Change in Health, which is scored separately. ${ }^{20}{ }^{22}$ Each domain has a scoring range of $0-100$, with a high score indicating better health or less body pain. The corresponding Physical (PCS) and Mental Component Summary (MCS) scores were also calculated. ${ }^{23}$ All findings were compared with reported SF-36 mean (SD) domains and summary values among healthy Dutch individuals (n 5 1742, including 976 men and 766 women). ${ }^{20,21}$

The 13-item Small-Fiber Neuropathy and Symptoms Inventory Questionnaire (SFN$\mathrm{SIQ}$ ), which assesses changes in sweating pattern, presence of diarrhea, constipation, urinary tract problems like hesitation and incontinence, dry eyes, dry mouth, dizziness when standing up, palpitations, hot flashes, sensitive leg skin, burning feet, sheet intolerance, and restless legs at night, was administered to each patient. Each item was scored on a 4-point Likert scale (0-never present; 1 - sometimes, 2-often, and 3-always present). A very similar scale has demonstrated discriminatory validity in patients with and without SFN suffering from sarcoidosis. ${ }^{28}$

Pain intensity was examined using a pain Visual Analog Scale (VAS pain current: the current presence of pain intensity; VAS pain minimum: the minimum level of pain intensity during the day; VAS pain maximum: the maximum level of pain intensity during the day). ${ }^{32}$

\section{Statistical Analysis}

Comparison Studies.

For the whole group and for the various subgroups, the mean SF-36 subscales and summary (PCS/ MCS) values were compared between the subgroups and with the reported mean normal values for the Dutch population (Student t-test for independent groups). ${ }^{20,21}$ Subgroups were based on test results: subgroup A, both IENFD and TTT abnormal; subgroup B, IENFD abnormal and TTT normal; subgroup C, IENFD normal and TTT abnormal; or etiological grouping: sarcoidosis subgroup (subgroup Sarc') versus other etiologies (subgroup Sarc 2); and finally "idiopathic" subgroup versus "nonidiopathic" subgroup. Subgroups of patients were examined to determine whether the patient group was homogeneous, and whether the etiology of SFN biased the study outcome.

Univariate and Multivariate Regression Studies.

Linear regression analyses were performed to determine which variables (age, gender, duration of symptoms prior to start of the study, SFN symptoms measured with SFN$S I Q$, VAS scores, explanatory variables) would have the greatest impact on patients' own PCS and MCS measures (dependent variables). The PCS was chosen, because it captures limitations in care, physical, social and role activities, amount of pain, and level of energy as a comprehensive overall score. The MCS measures the frequency of 
psychological distress and limitations in usual social and role activities due to emotional problems. ${ }^{23}$ Prior to the regression studies, the distribution patterns of the dependent PCS and MCS variables were examined and, if necessary, transformed to obtain a normal distribution pattern. Univariate regressions were performed subsequently, striving for the best fit between the dependent and each explanatory variable, separately. This was accomplished through systematic evaluation of constructed graphs with linear regression studies, including a restricted cubic spline function on the independent variable where possible. ${ }^{33}$ In addition, multivariate linear regressions were performed using a forward-adding stepwise strategy (significant level for adding set at 0.10 ) of explanatory variables on both the PCS and MCS scores, separately. The strength of association between the dependent variable and explanatory variables was presented as $R^{2}$, which is the fraction of variance explained by the independent variables from the regression model. The impact of age, gender, duration of symptoms, SFN-SIQ questions, and VAS scores was examined (in the multivariate setting) on each SF-36 domain separately (for the whole group of patients) to determine the strongest correlating domain of interest. All analyses were performed using Stata (version 12.0) for Windows XP. P $<0.05$ was considered significant.

\section{RESULTS}

\section{Patients}

A total of 315 patients were screened. Of these, 9 had large-fiber involvement at examination or on nerve conduction studies, 7 had incomplete data (refused skin biopsy or TTT, or poor compliance to TTT), and 34 had normal IENFD and TTT values. These 50 patients were excluded from the study. Eventually, 265 patients were diagnosed with SFN and entered in the study. The demographic and clinical features of these patients are presented in Tables 1 and 2. Approximately one-third (35.1\%) of the patients had both abnormal IENFD and TTT values (subgroup A, n 5 93). Most patients (57.7\%) had normal IENFD and abnormal TTT (subgroup C, n 5 153). No differences were seen between the subgroups regarding age, duration of symptoms, and number and severity of SFN complaints. The probable etiologies are also listed in Table 1. In most patients, SFN was associated with a systemic illness (particularly sarcoidosis) or remained idiopathic after thorough etiological screening. 
Table 1. Basic characteristics of patients with small-fiber neuropathy.

\begin{tabular}{|c|c|}
\hline & $\begin{array}{l}\text { Patients } \\
(n=265)\end{array}$ \\
\hline $\begin{array}{l}\text { Gender, n (\%) } \\
\text { Women } \\
\text { Men }\end{array}$ & $\begin{array}{l}137(51.7) \\
128(48.3)\end{array}$ \\
\hline Age in years: mean (SD), range & $50.2(12.6), 17-81$ \\
\hline Duration of symptoms in years: mean (SD), range & $7.6(9.9), 0-70$ \\
\hline $\begin{array}{l}\text { Etiology, } \mathrm{n}(\%) \\
\text { Idiopathic } \\
\text { Sarcoidosis/SLE1/Sjögren's syndrome } \\
\text { SCN9A² mutation } \\
\text { Diabetic } \\
\text { B6 intoxication } \\
\text { Paraproteinemia } \\
\text { Lyme } \\
\text { Alcohol induced } \\
\text { Fabry } \\
\text { Medication induced } \\
\text { Thyroid dysfunction } \\
\text { Celiac disease } \\
\text { Chronic Lymphatic Leukemia }\end{array}$ & $\begin{array}{c}102(38.5) \\
96 / 1 / 1(37.0) \\
17(6.4) \\
12(4.5) \\
6(2.3) \\
8(3.0) \\
3(1.1) \\
2(0.8) \\
1(0.4) \\
2(0.8) \\
6(2.3) \\
2(0.8) \\
1(0.4) \\
\end{array}$ \\
\hline VAS $^{3}$ pain score (amount of current pain), mean (SD), range & $47.8(28.8), 0-100$ \\
\hline VAS $^{3}$ pain score (lowest pain intensity), mean (SD), range & $26.6(22.2), 0-100$ \\
\hline VAS $^{3}$ pain score (highest pain intensity), mean (SD), range & $70.9(26.6), 0-100$ \\
\hline
\end{tabular}

\section{Chapter}

Table 2. SFN symptom inventory questionnaire findings in $n=265$ patients, scores $\%$ of responders.

\begin{tabular}{lcccc}
\hline & Never & Sometimes & Often & Always \\
\hline Changed sweating pattern & 53.2 & 62.3 & 16.6 & 6.4 \\
Diarrhea & 62.3 & 24.2 & 12.1 & 1.5 \\
Constipation & 62.6 & 21.5 & 13.2 & 2.6 \\
Micturation problems & 55.9 & 24.2 & 14.3 & 5.7 \\
Dry eyes & 60.8 & 18.5 & 15.5 & 5.3 \\
Dry mouth & 50.9 & 22.3 & 20.4 & 6.4 \\
Dizziness upon standing & 54.0 & 32.8 & 11.3 & 1.9 \\
Palpitations & 60.0 & 32.8 & 6.4 & 0.8 \\
Hot flashes & 57.0 & 23.4 & 17.4 & 2.3 \\
Sensitive skin & 48.3 & 14.3 & 15.5 & 21.9 \\
Burning feet & 41.1 & 11.7 & 20.8 & 26.4 \\
Sheet intolerance & 50.2 & 19.6 & 17.4 & 12.8 \\
Restless legs & 43.4 & 18.9 & 22.3 & 15.5 \\
\hline
\end{tabular}

\section{SF-36 Findings}

All SF-36 domains and summary scores were significantly $(P<0.0001)$ lower for the whole group of patients when compared with reported normative values for the healthy Dutch population (Figure 1). The domains Physical Functioning, Role Functioning-Physical, and Body Pain, and PCS demonstrated the lowest values in the SFN group (Figure 1). 
Figure 1. SF-36 findings in 265 patients compared to healthy Dutch controls.

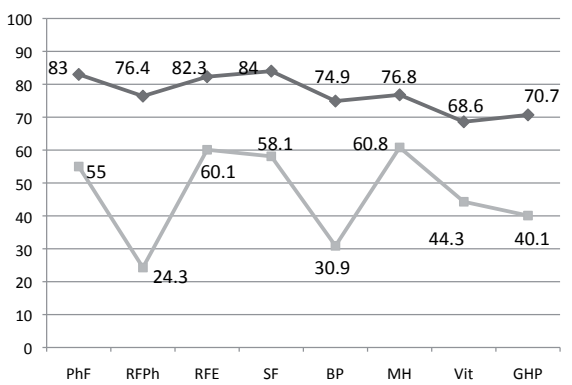

$\sim$ Healthy Dutch controls $(\mathrm{n}=1742) \quad-$ SFN patients $(\mathrm{n}=265)$

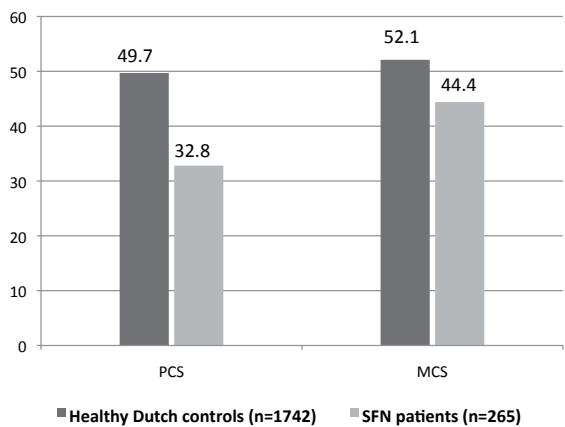

- Healthy Dutch controls $(\mathrm{n}=1742) \quad$ SFN patients $(\mathrm{n}=265)$

All domains and component summary of SF-36 findings were significantly lower in patients with small fiber neuropathy (SFN) when compared to corresponding values in healthy Dutch controls $(\mathrm{P}<0.0001$ for all comparisons). ${ }^{20,21}$

PhF, Physical Functioning; RFPh, Role Functioning-Physical; RFE, Role Functioning-Emotional; SF, Social Functioning; BP, Body Pain; MH, Mental health; Vit, Vitality; GHP, General Health perception; PCS, Physical Component Summary score, MCS, Mental Component Summary score.

Table 3. SF-36 health-related quality of life in patients with small fiber neuropathy.

\begin{tabular}{|c|c|c|c|c|c|c|}
\hline SF-36 Domain & \multicolumn{3}{|c|}{ Physical component summary score (PCS) } & \multicolumn{3}{|c|}{ Mental component summary score (MCS) } \\
\hline \multirow[t]{2}{*}{ A } & \multicolumn{6}{|c|}{ Subgroup $A$ versus $B$ versus $C$} \\
\hline & \multicolumn{3}{|c|}{ PCS } & \multicolumn{3}{|c|}{ MCS } \\
\hline Group & Mean & SD & Range & Mean & SD & Range \\
\hline$A(n=93)$ & 32.3 & 8.7 & $12.2-50.3$ & 43.2 & 11.3 & $22.6-72.5$ \\
\hline$B(n=19)$ & 32.1 & 11.8 & $13.8-60.3$ & 43.6 & 9.8 & $23-57.4$ \\
\hline$C(n=153)$ & 33.2 & 9.2 & $13.8-55.9$ & 45.3 & 10.7 & $20-75.8$ \\
\hline \multirow[t]{2}{*}{ B } & \multicolumn{6}{|c|}{$\begin{array}{l}\text { Patients with SFN related to sarcoidosis (Sarc+) versus } \\
\text { SFN patients with non-sarcoidosis etiology (Sarc-) }\end{array}$} \\
\hline & \multicolumn{3}{|c|}{ PCS } & \multicolumn{3}{|c|}{ MCS } \\
\hline Group & Mean & SD & Range & Mean & SD & Range \\
\hline Sarc $+(n=96)$ & 34.1 & 9.5 & $13.2-60.3$ & 45.3 & 10.5 & $20-72.5$ \\
\hline Sarc- $(n=169)$ & 31.9 & 9 & $12.2-53.3$ & 43.6 & 10.8 & $20.8-64.6$ \\
\hline \multirow[t]{2}{*}{ C } & \multicolumn{6}{|c|}{$\begin{array}{l}\text { Patients with idiopathic SFN (idSFN) versus } \\
\text { non-idiopathic SFN patients (nidSFN) }\end{array}$} \\
\hline & \multicolumn{3}{|c|}{ PCS } & \multicolumn{3}{|c|}{ MCS } \\
\hline Group & Mean & SD & Range & Mean & SD & Range \\
\hline idSFN $(n=102)$ & 32.3 & 8.4 & $13.8-49.5$ & 43.2 & 11.4 & $20.8-62.1$ \\
\hline nidSFN $(n=163)$ & 33 & 9.6 & $12.2-60.3$ & 45.1 & 10.5 & $20-75.8$ \\
\hline
\end{tabular}

PCS: Physical component summary score; MCS: Mental component summary score; SD: standard deviation Subgroup A: abnormal intra epidermal nerve fiber density (IENFD) and temperature threshold testing (TTT) ; Subgroup B: abnormal IENFD and normal TTT; Subgroup C: normal IENFD and abnormal TTT

Sarc+: small fiber neuropathy (SFN) associated with sarcoidosis; Sarc-: idiopathic SFN or associated with other etiology than sarcoidosis

idSFN: idiopathic SFN; nidSFN: non-idiopathic SFN

When the results of the various subgroups of patients (A, B, and C; subgroup $\operatorname{Sarc}^{1}$ and subgroup Sarc'; idiopathic vs. non-idiopathic subgroup) were compared with reported Dutch normative data, the scores remained significantly lower in the subgroups. No 
differences between subgroups A (both IENFD and TTT abnormal), B (only IENFD abnormal), and C (only TTT abnormal) were seen for the SF-36 domains and summary scores (Table 3, table 4 A). No differences in summary (PCS and MCS) scores were seen between the Sarc ${ }^{1}$ and $\operatorname{Sarc}^{2}$ subgroups. However, at the domain level, subgroup Sarc $^{2}$ demonstrated a lower mean score in the domains of Physical Functioning, Social Functioning, Body Pain (indicating more pain), and Mental Health when compared with subgroup Sarc ${ }^{1}$ (Table 4 B). The remaining domains and summary scores did not differ between these 2 subgroups. For all domains except 2 (Body Pain and General Health Perception) and for both summary (PCS and MCS) scores, no differences were seen between the idiopathic SFN subgroup and the non-idiopathic SFN subgroup (Table 4 C). The mean score for Body Pain was significantly lower in the idiopathic SFN subgroup. The mean score for General Health Perception was significantly lower in the non-idiopathic SFN subgroup (Table 4 C).

Table 4. SF-36 health-related quality of life in patients with small fiber neuropathy.

\begin{tabular}{|c|c|c|c|c|c|c|}
\hline A & \multicolumn{3}{|c|}{ Subgroups: A vs B vs C } & \multicolumn{3}{|c|}{ Subgroups: A vs B vs C } \\
\hline \multirow[t]{2}{*}{ SF-36 domain } & \multicolumn{3}{|c|}{ Mean } & \multicolumn{3}{|c|}{$\mathrm{SE}+95 \% \mathrm{Cl}$} \\
\hline & $A(n=93)$ & $B(n=19)$ & $C(n=153)$ & $A(n=93)$ & $B(n=19)$ & $C(n=153)$ \\
\hline Physical Functioning & 50.5 & 52.3 & 58.3 & $2.2(54-62.6)$ & $6.6(39.1-66.7)$ & $2.2(54-62.6)$ \\
\hline Role Functioning Physical & 22.5 & 30.3 & 24.7 & $3(18.8-30.5)$ & $9.7(9.9-50.6)$ & $3(18.8-30.5)$ \\
\hline Role Functioning Emotional & 53.7 & 64.9 & 63.4 & $3.6(56.4-70.5)$ & $9.7(44.5-85.3)$ & $3.6(56.4-70.5)$ \\
\hline Social Functioning & 56.2 & 46.7 & 60.9 & $2.3(56.2-65.4)$ & $6.7(32.5-60.9)$ & $2.3(56.2-65.4)$ \\
\hline Body Pain & 29.1 & 31.7 & 31.8 & $1.7(28.4-35.2)$ & $5.3(20.5-42.9)$ & $1.7(28.4-35.2)$ \\
\hline Mental Health & 59.7 & 62.2 & 61.2 & $1.4(58.5-64)$ & $3.5(54.9-69.6)$ & $1.4(58.5-64)$ \\
\hline Vitality & 43.1 & 41.9 & 43.9 & $1.9(41.6-49.2)$ & $3.7(34.2-49.7)$ & $1.9(41.6-49.2)$ \\
\hline General Health Perception & 39.8 & 42.9 & 40 & $1.5(37-43)$ & $5.4(31.5-54.3)$ & $1.5(37-43)$ \\
\hline $\begin{array}{l}\text { Physical Component } \\
\text { Summary Score }\end{array}$ & 32.3 & 32.1 & 33.2 & $0.8(31.6-34.8)$ & $2.8(26.3-38)$ & $0.8(31.6-34.8)$ \\
\hline $\begin{array}{l}\text { Mental Component } \\
\text { Summary Score }\end{array}$ & 43.2 & 43.6 & 45.3 & $0.9(43.5-47.2)$ & $2.3(38.7-48.4)$ & $0.9(43.5-47.2)$ \\
\hline A & Subgrc & oups: A vs & B vs $C$ & \multicolumn{3}{|c|}{ Subgroups: A vs B vs C } \\
\hline \multirow[t]{2}{*}{ SF-36 domain } & \multicolumn{3}{|c|}{ SD } & & Range & \\
\hline & $A(n=93)$ & $B(n=19)$ & $C(n=153)$ & $A(n=93)$ & $B(n=19)$ & $C(n=153)$ \\
\hline Physical Functioning & 26.3 & 28.6 & 25.3 & $0-100$ & $5-90$ & $0-100$ \\
\hline Role Functioning Physical & 34.6 & 42.1 & 35.6 & $0-100$ & $0-100$ & $0-100$ \\
\hline Role Functioning Emotional & 43.5 & 42.3 & 42.9 & $0-100$ & $0-100$ & $0-100$ \\
\hline Social Functioning & 31.1 & 29.4 & 28.1 & $0-100$ & $0-100$ & $0-100$ \\
\hline Body Pain & 19 & 22.6 & 20.7 & $0-100$ & $0-100$ & $0-100$ \\
\hline Mental Health & 16.7 & 14.8 & 16.5 & $24-88$ & $20-84$ & $12-88$ \\
\hline Vitality & 16.5 & 15.6 & 14.2 & $10-90$ & $10-75$ & $10-75$ \\
\hline General Health Perception & 20.8 & 22.9 & 18.3 & $0-87$ & $5-77$ & $5-97$ \\
\hline $\begin{array}{l}\text { Physical Component } \\
\text { Summary Score }\end{array}$ & 8.7 & 11.8 & 9.2 & $12.2-50.3$ & $13.8-60.3$ & $13.8-55.9$ \\
\hline $\begin{array}{l}\text { Mental Component } \\
\text { Summary Score }\end{array}$ & 11.3 & 9.8 & 10.7 & $22.6-72.5$ & $23-57.4$ & $20-75.8$ \\
\hline
\end{tabular}

Subgroup A: abnormal intra epidermal nerve fiber density (IENFD) and temperature threshold testing (TTT) Subgroup B: abnormal IENFD and normal TTT

Subgroup C: normal IENFD and abnormal TTT 
Table 4. Continued

\begin{tabular}{|c|c|c|c|c|}
\hline \multirow{3}{*}{$\begin{array}{l}\text { B } \\
\text { SF-36 domain }\end{array}$} & \multicolumn{4}{|c|}{$\begin{array}{l}\text { Patients with SFN related to sarcoidosis (Sarc+; } n=96 \text { ) versus } \\
\text { SFN patients with non-sarcoidosis etiology (Sarc-; } n=169 \text { ) }\end{array}$} \\
\hline & \multicolumn{2}{|c|}{ Subgroup Sarc $_{\text {pos }}$} & \multicolumn{2}{|c|}{ SubgroupSarc $_{\text {neg }}$} \\
\hline & Mean & SE $(95 \% \mathrm{Cl})$ & Mean & SE $(95 \% \mathrm{Cl})$ \\
\hline Physical Functioning ${ }^{1}$ & 60.2 & $2.5(55.3-65.0)$ & 51.7 & $2.2(47.3-56.0)$ \\
\hline Role Functioning Physical & 23.7 & $3.4(16.9-30.5)$ & 24.6 & $3(18.9-30.6)$ \\
\hline Role Functioning Emotional & 63.5 & $4.1(55.3-71.7)$ & 58.0 & $3.6(51-64.0)$ \\
\hline Social Functioning ${ }^{2}$ & 64.7 & $2.8(59.2-70.3)$ & 54.1 & $2.4(49.4-58.8)$ \\
\hline Body Pain ${ }^{3}$ & 38.2 & $2.2(33.8-42.7)$ & 26.2 & $1.4(23.4-29)$ \\
\hline Mental Health ${ }^{4}$ & 63.8 & $1.5(60.7-66.8)$ & 58.9 & $1.4(56.1-61.6)$ \\
\hline Vitality & 43.9 & $1.7(40.6-47.1)$ & 43.2 & $1.2(40.9-45.6)$ \\
\hline General Health Perception & 38.9 & $1.8(35.2-42.5)$ & 40.9 & $1.7(37.7-44.2)$ \\
\hline Physical Component Summary Score & 34.1 & $1(32.1-36)$ & 31.9 & $0.8(30.3-33.4)$ \\
\hline Mental Component Summary Score & 45.3 & $1.1(43.2-47.4)$ & 43.6 & $0.9(42-45.7)$ \\
\hline
\end{tabular}

\begin{tabular}{|c|c|c|c|c|}
\hline \multirow{3}{*}{$\begin{array}{l}\text { B } \\
\text { SF-36 domain }\end{array}$} & \multicolumn{4}{|c|}{$\begin{array}{l}\text { Patients with SFN related to sarcoidosis (Sarc }+; n=96) \text { versus } \\
\text { SFN patients with non-sarcoidosis etiology (Sarc-; } n=169 \text { ) }\end{array}$} \\
\hline & \multicolumn{2}{|c|}{ Subgroup Sarc $_{\text {pos }}$} & \multicolumn{2}{|c|}{ SubgroupSarc $_{\text {neg }}$} \\
\hline & SD & Range & SD & Range \\
\hline Physical Functioning ${ }^{1}$ & 24.1 & $5-100$ & 26.9 & $0-100$ \\
\hline Role Functioning Physical & 33.5 & $0-100$ & 37.0 & $0-100$ \\
\hline Role Functioning Emotional & 40.5 & $0-100$ & 44.7 & $0-100$ \\
\hline Social Functioning ${ }^{2}$ & 27.4 & $0-100$ & 23 & $0-100$ \\
\hline Body Pain ${ }^{3}$ & 21.9 & $0-100$ & 17.6 & $0-100$ \\
\hline Mental Health ${ }^{4}$ & 14.9 & $16-88$ & 17.1 & $12-88$ \\
\hline Vitality & 16.3 & $15-90$ & 14.4 & $10-75$ \\
\hline General Health Perception & 18 & $0-87$ & 20.5 & $5-97$ \\
\hline Physical Component Summary Score & 9.5 & $13.2-60.3$ & 9 & $12.2-53.5$ \\
\hline Mental Component Summary Score & 10.5 & $20.0-72.5$ & 10.8 & $20.8-64.6$ \\
\hline
\end{tabular}

SubgroupSarc $_{\text {pos }}$ :SFN associated with sarcoidosis versus SubgroupSarc : idiopathic SFN or associated with other etiology than sarcoidosis

T-tests performed: ${ }^{1} p=0.007 ;{ }^{2} p=0.003 ;{ }^{3} p<0.0001 ;{ }^{4} p=0.01$. P-values indicate significantly lower mean scores in the corresponding domains for the SubgroupSarc $c_{\text {neg }}$ when compared to the SubgroupSarc ${ }_{\text {pos }}$

\begin{tabular}{l|c|c|c|c}
\hline C & \multicolumn{3}{|c}{$\begin{array}{c}\text { Patients with idiopathic SFN (n=102) versus } \\
\text { non-idiopathic SFN patients (n=163) }\end{array}$} \\
\hline SF-36 domain & \multicolumn{3}{|c|}{ Idiopathic SFN subgroup } & \multicolumn{1}{c}{ Non-idiopathic SFN subgroup } \\
\hline & Mean & SE (95\% CI) & Mean & SE (95\% CI) \\
\hline Physical Functioning & 53.6 & $2.9(47.9-59.3)$ & 55.8 & $2.1(51.7-59.8)$ \\
\hline Role Functioning Physical & 26.4 & $4(18.4-34.3)$ & 23.1 & $2.7(17.8-28.5)$ \\
\hline Role Functioning Emotional & 58.1 & $4.7(48.7-67.4)$ & 61.3 & $3.3(54.7-67.8)$ \\
\hline Social Functioning & 54.4 & $3.3(47.9-60.9)$ & 60.2 & $2.2(55.9-64.6)$ \\
\hline Body Pain & 25.7 & $1.7(22.4-29)$ & 33.7 & $1.7(30.3-37.1)$ \\
\hline Mental Health & 58.9 & $1.9(55.2-62.7)$ & 61.8 & $1.2(59.4-64.3)$ \\
\hline Vitality & 44.1 & $1.7(40.8-47.4)$ & 43.1 & $1.2(40.8-45.5)$ \\
\hline General Health Perception ${ }^{1}$ & 43.3 & $2.2(38.8-47.7)$ & 38.4 & $1.5(35.5-41.3)$ \\
\hline Physical Component Summary Score & 32.3 & $0.9(30.4-34.2)$ & 33 & $0.8(31.5-34.5)$ \\
\hline Mental Component Summary Score & 43.2 & $1.3(40.7-45.8)$ & 45.1 & $0.8(43.4-46.7)$ \\
\hline
\end{tabular}


Table 4. Continued

\begin{tabular}{l|c|c|c|c}
\hline C & \multicolumn{3}{|c}{$\begin{array}{c}\text { Patients with idiopathic SFN (n=102) versus } \\
\text { Non-idiopathic SFN patients (n=163) }\end{array}$} \\
\hline SF-36 domain & \multicolumn{2}{|c|}{ Idiopathic SFN subgroup } & Non-idiopathic SFN subgroup \\
\hline & SD & Range & SD & Range \\
\hline Physical Functioning & 26.6 & $5-100$ & 25.9 & $0-100$ \\
\hline Role Functioning Physical & 37.9 & $0-100$ & 34.4 & $0-100$ \\
\hline Role Functioning Emotional & 45.3 & $0-100$ & 42 & $0-100$ \\
\hline Social Functioning & 31.3 & $0-100$ & 28.2 & $0-100$ \\
\hline Body Pain & 15.7 & $0-100$ & 21.9 & $0-100$ \\
\hline Mental Health & 17.7 & $20-88$ & 15.6 & $12-88$ \\
\hline Vitality & 15.5 & $10-75$ & 15 & $15-90$ \\
\hline General Health Perception ${ }^{2}$ & 21 & $5-87$ & 18.5 & $0-97$ \\
\hline Physical Component Summary Score & 8.4 & $13.8-49.5$ & 9.6 & $12.2-60.3$ \\
\hline Mental Component Summary Score & 11.4 & $20.8-62.1$ & 10.5 & $20-75.8$ \\
\hline
\end{tabular}

Patients with idiopathic SFN versus non-idiopathic SFN:

T-test performed: ${ }^{1} p=0.001$, meaning significantly more pain experienced in the idiopathic-SFN subgroup when compared to the non-idiopathic-SFN subgroup; ${ }^{2} \mathrm{p}=0.03$, indicating a lower mean general health perception in the non-idiopathic-SFN subgroup when compared to the idiopathic-SFN findings.

\section{Univariate Regression Studies on PCS and MCS}

The distribution patterns for the PCS and MCS were fairly normal. Age and duration of symptoms did not have a significant impact on PCS and MCS scores. Gender had a very minor impact on PCS (explaining 2\%) (Table 5). All SFN-SIQ inquiries demonstrated a very weak association with PCS, with each question explaining $<10 \%$ of PCS, except for Q6 (dry mouth; 13\% explained by this question) (Table 5). The pain VAS scores showed a slightly better explanation for PCS values (VAS pain current: $R^{2}=0.23$; VAS pain minimum: $R^{2}=0.21$; VAS pain maximum: $\left.R^{2}=0.21 ; P \leq 0.007\right)$. The VAS scores and some SFN-SIQ items (Q1, Q3, Q6, Q9, and Q10) were very weakly related to MCS, each explaining $<5 \%$ of the MCS findings (Table 5 ). The remaining items did not have any significant impact on MCS. Multivariate Regression Analyses on PCS and MCS. Through a stepwise-adding approach, a total of $32 \%\left(R^{2}=0.32\right)$ of PCS scores were explained by SFN-SIQ inquiry 06 (dry mouth), inquiry 01 (changes in sweating pattern), all VAS scores, and age (Table 5). The strongest predictor was SIQ 06 (dry mouth), showing a significant inverse correlation with PCS findings. Only 7\% of MCS could be explained by gender, VAS 03, and SFN-SIQ Q9, Q10, and Q12. The remaining items did not contribute to model on MCS (Table 5).

Multivariate Regression Analyses on SF-36 Domains. A multivariate regression stepwise adding analyses approach was performed on each individual SF-36 domain separately to determine which domain would have the strongest association with the explanatory variables (age, gender, duration of symptoms, SFN-SIQ, and VAS scores). The domain Body Pain showed a very strong association: a total of $43 \%\left[R^{2}=0.43, F(3.214)=55.95\right.$, $P<0.0001$ ] was explained by VAS 03 (the maximum level of pain intensity during the day; $\beta-7.71, P<0.001$ ), as did VAS 02 (the minimum level of pain intensity during the day; $\beta-3.55, P<0.001$ ) and $Q 7$ of the SFN-SIQ (dizziness when standing up; $\beta-2.41, P=$ 
0.017). The second best domain of interest being explained was Physical Functioning $\left[R^{2}=0.31, F(4,208)=25.24, P<0.0001\right.$ ], explained by Q1 (sweating pattern; $\beta 2.32, P=$ 0.022 ) and Q6 (dry mouth; $\beta-3.98, P<0.001)$ ) of the SFN-SIQ plus VAS $02(\beta-6.41, P<$ $0.001)$ and gender $(\beta 2.25, P=0.026)$.

Table 5. Uni- and Multi-variate regression studies on SF-36 Physical and Mental Component Summary Scores in patients with small fiber neuropathy.

\begin{tabular}{l|c|c|c|c|c|c}
\hline A & \multicolumn{5}{c}{ Physical Component Summary Score } \\
\hline SF-36 & \multicolumn{5}{c}{ Univariate regressions } & \multicolumn{2}{c}{$\begin{array}{c}\text { Multivariate regressions* } \\
\mathbf{R}^{*} \mathbf{0} \mathbf{0 . 3 2} \quad \mathbf{F}(\mathbf{6 . 2 0 0})=\mathbf{1 7} ; \boldsymbol{P}<\mathbf{0 . 0 0 0 1}\end{array}$} \\
\hline & $\mathbf{R}^{2}$ & $\mathbf{F}$ & $\boldsymbol{\beta}$ & $\boldsymbol{\beta}$ & $\boldsymbol{P}$-value & $\mathbf{R}^{\mathbf{2}}$ \\
\hline Age & 0.0001 & $(1,232)=0.02$ & $0.14, p=0.89$ & 2.07 & 0.04 & 0.004 \\
\hline Gender & 0.02 & $(1,232)=5.01$ & $2.24, p=0.026$ & & & 0.004 \\
\hline Symptom duration & 0.003 & $(1,218)=0.38$ & $-0.62, p=0.537$ & & & 0.003 \\
\hline VAS pain current & 0.23 & $(1,228)=69.06$ & $-8.31, p<0.001$ & -1.46 & 0.147 & 0.04 \\
\hline VAS pain minimum & 0.20 & $(1,224)=56.20$ & $-7.50, p<0.001$ & -2.12 & 0.036 & 0.04 \\
\hline VAS pain maximum & 0.21 & $(1,222)=59.37$ & $-7.71, p<0.001$ & -2.68 & 0.008 & 0.04 \\
\hline Changed sweating & 0.02 & $(1,232)=6.10$ & $-2.47, p=0.014$ & 2.90 & 0.004 & 0.02 \\
\hline Diarrhea & 0.02 & $(1,232)=5.71$ & $-2.39, p=0.018$ & & & 0.001 \\
\hline Constipation & 0.03 & $(1,232)=9.43$ & $-3.07, p=0.002$ & & & 0.03 \\
\hline Micturation problem & 0.06 & $(1,232)=15.78$ & $-3.97, p<0.001$ & & & 0.006 \\
\hline Dry eyes & 0.05 & $(1,232)=13.41$ & $0.05, p<0.001$ & & & 0.002 \\
\hline Dry mouth & 0.13 & $(1,232)=36.08$ & $-6.01, p<0.001$ & -3.53 & 0.001 & 0.01 \\
\hline Dizziness standing & 0.07 & $(1,232)=17.53$ & $-4.19, p<0.001$ & & & 0.0002 \\
\hline Palpitations & 0.08 & $(1,232)=20.75$ & $-4.56, p<0.001$ & & & 0.0002 \\
\hline Hot flashes & 0.05 & $(1,232)=12.28$ & $-3.5, p=0.001$ & & & 0.03 \\
\hline Sensitive skin & 0.06 & $(1,232)=16.74$ & $-4.09, p<0.001$ & & & 0.03 \\
\hline Burning feet & 0.05 & $(1,232)=13.90$ & $-3.73, p<0.001$ & & & 0.009 \\
\hline Sheet intolerance & 0.07 & $(1,232)=19.73$ & $-4.44, p<0.001$ & & & 0.005 \\
\hline Restless legs & 0.06 & $(1,232)=15.34$ & $-3.92, p<0.001$ & & & 0.01 \\
\hline
\end{tabular}

\begin{tabular}{|c|c|c|c|c|c|c|}
\hline \multirow{4}{*}{$\begin{array}{l}\text { B } \\
\text { SF-36 }\end{array}$} & \multicolumn{6}{|c|}{ Mental Component Summary Score } \\
\hline & \multirow{2}{*}{\multicolumn{3}{|c|}{ Univariate regressions }} & \multirow{2}{*}{\multicolumn{3}{|c|}{$\begin{array}{l}\text { Multivariate regressions* } \\
\mathrm{R}^{2}: 0.07 \quad \mathrm{~F}(5,201)=4.26, P=0.001\end{array}$}} \\
\hline & & & & & & \\
\hline & $\mathbf{R}^{2}$ & $\mathrm{~F}$ & $\mathbf{R}^{2}$ & $\mathrm{~F}$ & $\mathbf{R}^{2}$ & $\mathrm{~F}$ \\
\hline Age & 0.004 & $(1,232)=0.02$ & 0.004 & $(1,232)=0.02$ & 0.004 & $(1,232)=0.02$ \\
\hline Gender & 0.004 & $(1,232)=0.05$ & 0.004 & $(1,232)=0.05$ & 0.004 & $(1,232)=0.05$ \\
\hline Symptom duration & 0.003 & $(1,218)=0.27$ & 0.003 & $(1,218)=0.27$ & 0.003 & $(1,218)=0.27$ \\
\hline VAS pain current & 0.04 & $(1,228)=11.17$ & 0.04 & $(1,228)=11.17$ & 0.04 & $(1,228)=11.17$ \\
\hline VAS pain minimum & 0.04 & $(1,224)=10.53$ & 0.04 & $(1,224)=10.53$ & 0.04 & $(1,224)=10.53$ \\
\hline VAS pain maximum & 0.04 & $(1,222)=9.54$ & 0.04 & $(1.222)=9.54$ & 0.04 & $(1.222)=9.54$ \\
\hline Changed sweating & 0.02 & $(1,232)=5.74$ & 0.02 & $(1,232)=5.74$ & 0.02 & $(1,232)=5.74$ \\
\hline Diarrhea & 0.001 & $(1,232)=0.68$ & 0.001 & $(1,232)=0.68$ & 0.001 & $(1,232)=0.68$ \\
\hline Constipation & 0.03 & $(1,232)=9.40$ & 0.03 & $(1,232)=9.40$ & 0.03 & $(1,232)=9.40$ \\
\hline Micturation problem & 0.006 & $(1,232)=2.31$ & 0.006 & $(1,232)=2.31$ & 0.006 & $(1,232)=2.31$ \\
\hline Dry eyes & 0.002 & $(1,232)=0.47$ & 0.002 & $(1,232)=0.47$ & 0.002 & $(1,232)=0.47$ \\
\hline Dry mouth & 0.01 & $(1,232)=3.99$ & 0.01 & $(1,232)=3.99$ & 0.01 & $(1,232)=3.99$ \\
\hline Dizziness standing & 0.0002 & $(1,232)=1.04$ & 0.0002 & $(1,232)=1.04$ & 0.0002 & $(1,232)=1.04$ \\
\hline Palpitations & 0.0002 & $(1,232)=0.96$ & 0.0002 & $(1,232)=0.96$ & 0.0002 & $(1,232)=0.96$ \\
\hline Hot flashes & 0.03 & $(1,232)=7.44$ & 0.03 & $(1,232)=7.44$ & 0.03 & $(1,232)=7.44$ \\
\hline Sensitive skin & 0.03 & $(1,232)=8.34$ & 0.03 & $(1,232)=8.34$ & 0.03 & $(1,232)=8.34$ \\
\hline Burning feet & 0.009 & $(1,232)=2.99$ & 0.009 & $(1,232)=2.99$ & 0.009 & $(1,232)=2.99$ \\
\hline Sheet intolerance & 0.005 & $(1,232)=2.06$ & 0.005 & $(1,232)=2.06$ & 0.005 & $(1,232)=2.06$ \\
\hline Restless legs & 0.01 & $(1,232)=3.85$ & 0.01 & $(1,232)=3.85$ & 0.01 & $(1,232)=3.85$ \\
\hline
\end{tabular}

*Only those items that remained in the multivariate model are reported.

VAS = visual analogue scale; VAS pain current: the current pain intensity; VAS pain minimum: the minimum pain intensity during the day, VAS pain maximum: the maximum pain intensity during the day. 


\section{DISCUSSION}

We have examined the impact of SFN on QOL. Using the SF-36 generic tool, there was a generally severe reduction in all domains and component summary scores compared with reported normative healthy Dutch control values (Figure 1). In particular, the domains Role Functioning-Physical, Body Pain, and PCS had lower scores, indicating a worse physical condition. ${ }^{20,23}$ The disability caused by polyneuropathy has been shown to correlate with a decrease in QOL. ${ }^{34}$ The body pain scores were also significantly lower, thus demonstrating severe SFN-related neuropathic pain, which conforms with earlier reports., 13, 15,35 It is known that painful polyneuropathy leads to a more severe reduction in QOL when compared with painless neuropathy. ${ }^{36-39}$

Also, patients with a disease complicated by the presence of peripheral polyneuropathy may show a reduced QOL that cannot be explained merely by the disease alone..40,41 In fact, the idiopathic SFN subgroup hardly showed differences from the non-idiopathic subgroup of patients, implying that the underlying illness that may lead to SFN did not have much impact on reduction of QOL (Table 3 and Tables $4 \mathrm{~A}-\mathrm{C}$ ). In a recent study involving various forms of peripheral neuropathies, similar SF-36 Body Pain findings were seen in patients classified as having pain. However, the PCS values in our patients were notably lower when compared with the reported scores. 42

The SF-36 values in our SFN population were also substantially lower than in other chronic diseases, such as myocardial infarction and angina pectoris with hypertension, as has been reported in the USA population by those who established the SF-36. ${ }^{22}$ Even the mental condition in our SFN patients was significantly reduced. This is in contrast to literature findings, which suggests an unaltered mental state in chronic conditions, including various forms of peripheral neuropathy. ${ }^{42-45}$ This is usually explained by adaptation or development of coping mechanisms over the years. Apparently, patients with SFN have ongoing, sometimes excruciating pain and experience continuous difficulty dealing with the consequences of their illness.

With respect to the objectives of this study, there are some methodological issues that should be addressed. First, because only $32 \%$ of PCS values were explained by SFN-related complaints, including pain, future studies should focus on other factors that may contribute to lower QOL in to contribute to decreased QOL in these patients. Factors like anxiety, depression, disturbed sleep, and fatigue have been demonstrated to contribute to decreased QOL in peripheral neuropathies. ${ }^{42,46,47}$ Second, because the MUMC is referral center for patients with sarcoidosis, our study population was biased toward this illness. The SF-36 component summary findings in the sarcoidosis group versus the other etiological SFN patients did not differ; however, some differences were seen at the domain level (Table 4 B). Despite this etiological bias, the findings in the sarcoidosis subgroup demonstrated approximately the same differences with the healthy Dutch community as seen in Figure 1. Similar findings were seen for the idiopathic versus non-idiopathic subgroup of patients. Therefore, we consider the 
findings from the total SFN group representative of SFN in general. Understanding the disabling complaints leading to a reduced QOL is essential in optimizing the guidance and hopefully the therapeutic approach in this condition. Third, we used a generic QOL metric, because our aim was to compare the findings with reported normative healthy community scores. The SF-36 is not a disease-specific tool, and perhaps a more specific questionnaire, such as the Vickrey 97-QoL scale, would have shown better targeting with stronger associations with the SFN complaints in our study population. ${ }^{48}$ The percentage of the physical summary score explained is, however, is slightly lower than that the reported explanation (40\%) in other peripheral neuropathies with more physical impair ments..$^{49,50}$

Because QOL may complement traditional outcome measures, a limited correlation is not unexpected. ${ }^{50}$ In conclusion, SFN has an overall severe impact on QOL, both physically and mentally. Some SFN symptoms, including pain, had an inverse correlation with QOL scores, explaining about one-third of physical QOL findings. Future studies are warranted to determine which additional factors may influence QOL in SFN. 


\section{REFERENCES}

1. Hoeijmakers JG, Faber CG, Lauria G, Merkies IS, Waxman SG. Small-fibre neuropathies-advances in diagnosis, pathophysiology and management. Nat Rev Neurol 2012;8:369-379.

2. Lacomis D. Small-fiber neuropathy. Muscle Nerve 2002;26:173-188.

3. Danaei G, Finucane MM, Lu Y, et al. National, regional, and global trends in fasting plasma glucose and diabetes prevalence since 1980: systematic analysis of health examination surveys and epidemiological studies with 370 country-years and 2.7 million participants. Lancet 2011;378:31-40.

4. Schmader KE. Epidemiology and impact on quality of life of postherpetic neuralgia and painful diabetic neuropathy. Clin J Pain 2002;18:350-354.

5. Daousi C, MacFarlane IA, Woodward A, Nurmikko TJ, Bundred PE, Benbow SJ. Chronic painful peripheral neuropathy in an urban community: a controlled comparison of people with and without diabetes. Diabet Med 2004;21:976-982.

6. Vlckova-Moravcova E, Bednarik J, Belobradkova J, Sommer C. Small-fibre involvement in diabetic patients with neuropathic foot pain. Diabet Med 2008;25:692-699.

7. Lauria G, Hsieh ST, Johansson O, et al. European Federation of Neurological Societies/Peripheral Nerve Society Guideline on the use of skin biopsy in the diagnosis of small fiber neuropathy. Report of a joint task force of the European Federation of Neurological Societies and the Peripheral Nerve Society. Eur J Neurol 2010;17:903-912, e944-909.

8. Holland NR, Stocks A, Hauer P, Cornblath DR, Griffin JW, McArthur JC. Intraepidermal nerve fiber density in patients with painful sensory neuropathy. Neurology 1997;48:708-711.

9. Holland NR, Crawford TO, Hauer P, Cornblath DR, Griffin JW, MCArthur JC. Small-fiber sensory neuropathies: clinical course and neuropathology of idiopathic cases. Ann Neurol 1998:44:47-59.

10. Said G. Small fiber involvement in peripheral neuropathies. Curr Opin Neurol 2003;16:601-602.

11. Sommer C, Lauria G. Chapter 41 Painful small-fiber neuropathies. Handbook of Clinical neurology2006: 621-633.

12. Lauria G. Small fibre neuropathies. Curr Opin Neurol 2005;18:591-597.

13. Devigili G, Tugnoli V, Penza P, et al. The diagnostic criteria for small fibre neuropathy: from symptoms to neuropathology. Brain 2008;131:1912-1925

14. Hoitsma E, Reulen JP, de Baets M, Drent M, Spaans F, Faber CG. Small fiber neuropathy: a common and important clinical disorder. J Neurol Sci 2004;227:119-130.

15. Tesfaye S, Boulton AJ, Dyck PJ, et al. Diabetic neuropathies: update on definitions, diagnostic criteria, estimation of severity, and treatments. Diabetes Care 2010;33:2285-2293.

16. Lauria G, Lombardi R. Skin biopsy: a new tool for diagnosing peripheral neuropathy. Bmj 2007;334:11591162.

17. Jamal GA, Hansen S, Weir Al, Ballantyne JP.The neurophysiologic investigation of small fiber neuropathies. Muscle Nerve 1987;10:537-545.

18. Nebuchennykh M, Loseth S, Lindal S, Mellgren SI. The value of skin biopsy with recording of intraepidermal nerve fiber density and quantitative sensory testing in the assessment of small fiber involvement in patients with different causes of polyneuropathy. J Neurol 2009;256:1067-1075.

19. Vlckova-Moravcova E, Bednarik J, Dusek L, Toyka KV, Sommer C. Diagnostic validity of epidermal nerve fiber densities in painful sensory neuropathies. Muscle Nerve 2008;37:50-60.

20. Aaronson NK, Muller M, Cohen PD, et al. Translation, validation, and norming of the Dutch language version of the SF-36 Health Survey in community and chronic disease populations. J Clin Epidemiol 1998;51:1055-1068.

21. Ware JE, Jr., Gandek B, Kosinski M, et al. The equivalence of SF-36 summary health scores estimated using standard and country-specific algorithms in 10 countries: results from the IQOLA Project. International Quality of Life Assessment. J Clin Epidemiol 1998;51:1167-1170.

22. Ware JE, jr., Snow KK, Kosinski M, Gandek B. SF-36 health survey. Manual and interpretation guide. Boston: The health institue, New England Medical Center, 1993.

23. Ware JE, Kosinski M, Keller SD. SF-36 physical and mental health summary scales: a user's manual. Boston: The Health Assessment Lab, New England Medical Center, 1994. 
24. Lauria G, Bakkers M, Schmitz C, et al. Intraepidermal nerve fiber density at the distal leg: a worldwide normative reference study. J Peripher Nerv Syst 2010;15:202-207.

25. Yarnitsky D, Sprecher E. Thermal testing: normative data and repeatability for various test algorithms. J Neurol Sci 1994;125:39-45.

26. Hoitsma E, Drent M, Verstraete $E$, et al. Abnormal warm and cold sensation thresholds suggestive of small-fibre neuropathy in sarcoidosis. Clin Neurophysiol 2003;114:2326-2333.

27. Martina IS, van Koningsveld R, Schmitz PI, van der Meche FG, van Doorn PA. Measuring vibration threshold with a graduated tuning fork in normal aging and in patients with polyneuropathy. European Inflammatory Neuropathy Cause and Treatment (INCAT) group. J Neurol Neurosurg Psychiatry 1998;65:743-747.

28. Bakkers M, Merkies IS, Lauria G, et al. Intraepidermal nerve fiber density and its application in sarcoidosis Neurology 2009;73:1142-1148.

29. Reulen JP, Lansbergen MD, Verstraete E, Spaans F. Comparison of thermal threshold tests to assess small nerve fiber function: limits vs. levels. Clin Neurophysiol 2003;1 14:556-563.

30. Shy ME, Frohman EM, So YT, et al. Quantitative sensory testing: report of the Therapeutics and Technology Assessment Subcommittee of the American Academy of Neurology. Neurology 2003;60:898-904.

31. Yarnitsky D, Sprecher E, Zaslansky R, Hemli JA. Heat pain thresholds: normative data and repeatability. Pain 1995;60:329-332

32. Maxwell C. Sensitivity and accuracy of the visual analogue scale: a psycho-physical classroom experiment. Br J Clin Pharmacol 1978;6:15-24.

33. Herndon JE, Jr. FEH. The restricted cubic spline hazard model. Communications in statistics: theory and methods 1990:639-663.

34. Padua L, Schenone A, Aprile I, et al. Quality of life and disability assessment in neuropathy: a multicentre study. J Peripher Nerv Syst 2005;10:3-10.

35. Tavee J, Zhou L. Small fiber neuropathy: A burning problem. Cleve Clin J Med 2009;76:297-305.

36. Van Acker K, Bouhassira D, De Bacquer D, et al. Prevalence and impact on quality of life of peripheral neuropathy with or without neuropathic pain in type 1 and type 2 diabetic patients attending hospital outpatients clinics. Diabetes Metab 2009;35:206-213.

37. Erdmann PG, van Genderen FR, Teunissen LL, et al. Pain in patients with chronic idiopathic axonal polyneuropathy. Eur Neurol 2010;64:58-64.

38. Hughes RA, Umapathi T, Gray IA, et al. A controlled investigation of the cause of chronic idiopathic axonal polyneuropathy. Brain 2004;127:1723-1730.

39. Liedberg GM, Vrethem M. Polyneuropathy, with and without neurogenic pain, and its impact on daily life activities--a descriptive study. Disabil Rehabil 2009;31:1402-1408.

40. Kim BJ, Park HR, Roh HJ, et al. Chemotherapy-related polyneuropathy may deteriorate quality of life in patients with B-cell lymphoma. Qual Life Res 2010;19:1097-1103.

41. Ku do Y, Park YS, Chang HJ, Kim SR, Ryu JW, Kim WJ. Depression and life quality in chronic renal failure patients with polyneuropathy on hemodialysis. Ann Rehabil Med 2012;36:702-707.

42. Poliakov I, Toth C. The impact of pain in patients with polyneuropathy. Eur J Pain 2011;15:1015-1022.

43. Kempen Gl, Ormel J, Brilman El, Relyveld J. Adaptive responses among Dutch elderly: the impact of eight chronic medical conditions on health-related quality of life. Am J Public Health 1997;87:38-44.

44. Lindh J, Tondel M, Persson B, Vrethem M. Health-related quality of life in patients with cryptogenic polyneuropathy compared with the general population. Disabil Rehabil 2011;33:617-623.

45. Teunissen LL, Eurelings M, Notermans NC, Hop JW, van Gijn J. Quality of life in patients with axonal polyneuropathy. J Neurol 2000;247:195-199.

46. Merkies IS, Schmitz PI, Samijn JP, van der Meche FG, van Doorn PA. Fatigue in immune-mediated polyneuropathies. European Inflammatory Neuropathy Cause and Treatment (INCAT) Group. Neurology 1999;53:1648-1654.

47. Viala-Danten M, Martin S, Guillemin I, Hays RD. Evaluation of the reliability and validity of the Medical Outcomes Study sleep scale in patients with painful diabetic peripheral neuropathy during an international clinical trial. Health Qual Life Outcomes 2008;6:113.

48. Vickrey BG, Hays RD, Beckstrand M. Development of a health-related quality of life measure for peripheral neuropathy. Neurorehabil Neural Repair 2000;14:93-104. 
49. Merkies IS, Bril V, Dalakas MC, et al. Health-related quality-of-life improvements in CIDP with immune globulin IV 10\%: the ICE Study. Neurology 2009;72:1337-1344.

50. Merkies IS, Schmitz PI, van der Meche FG, Samijn JP, van Doorn PA. Quality of life complements traditional outcome measures in immune-mediated polyneuropathies. Neurology 2002;59:84-91. 

PART IV

\section{SUMMARY, GENERAL DISCUSSION, AND \\ FUTURE PERSPECTIVES}




\section{Chapter 8.1}

\section{SUMMARY AND \\ GENERAL DISCUSSION}

This thesis comprises of a series of studies on small fiber neuropathy (SFN). The following will summarize the findings of the studies presented in this thesis. We will discuss the potential impact of these findings, as well as implications for future research.

\section{Introduction}

Chapter one is a general introduction, presenting general and clinical information on SFN, as well as the outline of this thesis. Patients with SFN may experience spontaneous pain and/or autonomic symptoms due to dysfunction of A-delta and C fibers. A combination of symptoms, signs and neurophysiologic and pathologic findings is used to confirm suspected SFN. There are numerous diseases associated with the occurrence of SFN, such as toxic, metabolic, inflammatory, and neoplastic disorders. Mutations in voltage gated sodium channels may also lead to SFN. ${ }^{1-3}$

\section{Diagnosis of small fiber neuropathy}

No gold standard for diagnosing SFN exists, unfortunately. Pathologic changes of both large and small nerve fibers can be examined in sural nerve biopsies, but this is an invasive procedure and is generally only recommended when amyloidisis or vasculitis is suspected. ${ }^{4}$ Various diagnostic tests have therefore been developed to examine specific functions of the nervous system (sensory threshold tests, evoked potentials, sudomotor and cardiovascular reflex testing). However, these may be subjective, difficult to perform or have limited sensitivity or specificity. ${ }^{5}$

In SFN, skin biopsy to determine the intraepidermal nerve fiber density (IENFD) is considered an objective diagnostic test. With a small $3 \mathrm{~mm}$ punch biopsy, skin from an affected or standard skin area (10 cm above the lateral malleolus) can be examined. This is a minimally invasive procedure, with little to no side effects on patients. ${ }^{6}$

In chapter 2.1, normative data for intraepidermal nerve fiber density (IENFD) are presented. First, data from a healthy Dutch and Italian cohort of subjects were 
gathered and examined. An age-related decrease in IENFD was found with lower densities in men compared to women. Clinical applicability was demonstrated in a cohort of sarcoidosis patients. Subsequently, an international collaborative effort was performed between eight skin biopsy laboratories from Europe, USA, and Asia. This has resulted in reference values, applicable world-wide with validation of the findings at our institute (chapter 2.2). In conclusion, the use of IENFD in clinical practice is made possible through studies that provide age- and gender-matched normative values.

Though the technique of taking a skin biopsy is fairly simple and minimally invasive, the fixation, staining and counting procedure is somewhat time consuming, and not available in all pathological laboratories. Moreover, due to the normal variability of IENFD, the sensitivity of this test is probably moderate. Therefore, the diagnosis of SFN usually requires a combination of IENFD with other tests.

Chapter 3.1 reviews the current use of temperature threshold testing (TTT), a quantified technique, more widely available, and often used in the evaluation of SFN. Great variety is seen, for example, in use of equipment, test modalities, testing algorithms, and reported values. Thus, the review shows lack of international standardization when applying this technique. Practical literature-based recommendations are made: to test for warm and cold sensation thresholds, the use of age- and gender-stratified normative values, testing with a reaction time-independent technique. Additionally, the responsiveness of this technique needs to be determined in clinical studies.

Using patients' data, the optimization of an extensive TTT protocol with high burden for patients and technicians showed a possible reduction of the protocol used, maintaining high sensitivity and specificity (chapter 3.2). The best reduced protocol found was testing both warm and cold, feet and hands bilaterally with the method of levels and in a reaction time independent manner. Of course, a patient is still required to be cooperative, highly concentrated and the test result is subjective so malingering cannot be excluded completely?

Contact heat and laser evoked potentials theoretically overcome some of these drawbacks as one would not easily expect an influence of the patients preferred outcome on evoked potentials. Chapter $\mathbf{4}$ addresses the recruitment of normative values for contact heat evoked potentials (CHEPs) in a large Dutch cohort. Latencies were influenced by height, amplitudes by gender. An age related decrease was seen for amplitudes with slightly increasing latencies. Stratified normative values were obtained that can be used to evaluate CHEP results in patients.

Unfortunately, CHEPs shows some practical problems. In some healthy subjects no potentials could be recorded, as was also previously described by other authors, 
resulting in a lower specificity. It is however, conceivable that some "healthy" subjects may have subclinical sensory deficit leading to abnormalities found when examining them with the CHEPS. Also, in our patients we noticed a more readily visible evoked potential when using a higher starting temperature, but a varying starting temperature may induce changes in latency and possibly amplitude. Standardization of the CHEPS test for clinical use should therefore be part of future studies, aiming to standardize the use of these devices, and determine the additional value of CHEPS in the diagnosis of SFN. We showed a diagnostic accuracy of 74\% for both TTT and CHEPS in patients with abnormal IENFD, but the relation of CHEPS to IENFD and TTT has to be elucidated in more detail. Furthermore, measuring CHEPs is experienced by the patients as being more painful than for example a skin biopsy, which may also have a disadvantage in clinical practice.

\section{Clinical and clinimetric aspects of small fiber neuropathy}

The existence of peripheral neuropathies (predominantly) involving small nerve fibers has been recognized for decades now, ${ }^{8}$ but focus has often been on diabetic neuropathies, probably because of the sheer volume of patients affected. The past years, interest in and the search for underlying causes including the need for more specific treatment has evolved. Small fiber neuropathy probably is not uncommon and patients may benefit from a diagnosis, even if no underlying cause is identified and no specific treatment is yet available. We have experienced patients being treated for a wrong diagnosis (e.g. psychiatric behavioral therapy) for years before being classified as having small fiber neuropathy.

Chapter

8.1

An estimated minimum incidence and prevalence of SFN was presented in chapter $\mathbf{5}$, for the catchment area of the Maastricht University Medical Center. This showed a minimum estimated incidence of $12 / 100.000$ and prevalence of 53/100.000, which is a substantial number, especially taking into account that true incidence and prevalence are probably higher as these were all referred patients. Awareness of SFN and related complaints hopefully improve in future.

Better identification of painful neuropathies and proper recognition of autonomic complaints, as part of the spectrum of polyneuropathies, is important. Both for patients and doctors, as this may influence diagnostic and treatment strategies and provide adequate explanation of symptoms.

In chapter $\mathbf{6}$ symptoms of SFN, pain and autonomic dysfunction, were evaluated by questionnaires in a group of sarcoidosis patients with and without symptoms indicative of small fiber neuropathy. Autonomic symptoms were reported in all patients, but more in those suspected of SFN (as this was based on a history of pain and autonomic complaints). Pain was also reported by all patients, but most severe in patients with reduced IENFD and more specifically for typically neuropathic subtypes. This shows 
that scales may help to appreciate the extent of patients' problems and may even help identifying those with SFN.

Chapter seven investigates the impact of SFN on quality of life. All patient experience a severe overall reduction in quality of life, and pain only explains part of this reduction. In this study, the SF36, a generic health-related quality of life scale was used and compared with reported Dutch normative data. The impact of having small fiber neuropathy is generally substantial, showing low scores that can be compared with patients that recently had a myocardial infarction. Pain and autonomic symptoms explained 32\% of the reduction in quality of life. This implies other important aspects such as disturbed sleep, fatigue, mood disorders, as well.

Overall, we can conclude that interest in (patients with) SFN is not a luxury, but a necessity. 


\section{Chapter 8.2}

\section{FUTURE PERSPECTIVES}

\section{Diagnosis of SFN}

The lack of gold standard for diagnosing SFN will possibly remain a challenge. Though clinicians nowadays adopt each other's criteria for definition and diagnosis, ${ }^{9}$ current use of TTT and skin biopsy as confirmatory tests both have limited sensitivity and specificity. There is a need for additional reliable, valid and responsive instruments to diagnose SFN.

Nerve conduction studies are only applicable to determine the presence or absence of large fiber involvement, and by definition NCS should be normal in SFN. As discussed above, there are several drawbacks to TTT: it is subjective, hard to compare between centers that use different protocols, reliability depends on testing method and location, and validity and responsiveness still need to be determined. Though evoked potentials, such as laser evoked potentials and CHEPS, seem a promising alternative, the examination is sometimes painful, and technique and results may be more difficult to implement in daily practice.

Microneurography is a specialized technique that provides information on small sensory and autonomic nerve fibers. With a metal microelectrode, inserted directly into a peripheral nerve, afferent and efferent nerve discharges can be recorded. A lot of research has been dedicated to sympathetic microneurography as an investigative tool in autonomic neural control. ${ }^{10,11}$ Afferent nociceptive discharges from C-fibers can also be recorded and hyper-excitability and sensitization of C-fibers has been shown in painful neuropathy. ${ }^{12}$ This technique may provide insight in nerve (dis)function, as in other chronic pain states such as fibromyalgia, in which hyper-excitability and sensitization have also been shown. ${ }^{13}$ However, the technique is not widely known in the neurology field and only a handful of medical doctors are trained to perform these recordings. Also, the examination is time consuming and today, not many patients have been examined yet for this purpose. It is also unknown what normative values would be in relation to age and gender. Therefore at current stage, the value of this test in the diagnosis of SFN is uncertain, but proves in interesting tool in a research setting. Though IENFD determination through skin biopsy is reliable and valid, responsiveness needs to be determined if this technique is considered as an outcome measure in 
clinical studies. Also, it is a slightly invasive test procedure with limited availability. Corneal confocal microscopy may be a suitable alternative. After local anesthesia, a lens of the confocal microscope is placed on the cornea. With a digital camera, a set of images can be captured and analyzed afterwards. This technique does require a specialized microscope for in vivo examination of the eye. However, such a microscope has several purposes in ophthalmologic practice and it allows for non-invasive examination of corneal nerve fiber density. Encouraging data are emerging suggesting corneal fiber density to be equally useful to IENFD. ${ }^{14-16}$

Probably, a combination of tests, possibly with a stepwise approach, will eventually be advised to confirm SFN diagnosis. Plans are underway to address these issues in an international workshop on the diagnostic approach in small fiber neuropathy, aiming for general consensus.

\section{Clinical and clinimetric aspects of small fiber neuropathy}

As stated before, even when no treatment is available (yet), patients may benefit from timely recognition of having SFN. ${ }^{17}$ Therefore, valid and reliable screening instruments and questionnaires are needed to identify patients that may potentially have SFN. These measures should preferably also be responsive if they are planned to be used in clinical studies. ${ }^{18}$ Most scales used in peripheral neuropathy are mainly composite multi-item measures at the ordinal level and are still being used, despite their generally known shortcomings. ${ }^{19-21}$

In essence, ordinal based measures are qualitative descriptive data with no numerical value. Modern methods like the Rasch methodology is gaining interest as a way to examine, improve, and create questionnaires. ${ }^{22,23}$ "The Rasch model states that the probability of a patient being able to correctly answer or complete an item or task is a logistic function of the difficulty of the task and the ability of the patient to acclompish it". For several neuromuscular diseases, specific outcome scales have been produced this way. ${ }^{24-26}$ Using the same frame, a Rasch-built overall disability scale specifically designed for SFN will be constructed fulfilling all clinimetrical requirements.

Pain experiences can be further differentiated to evaluate the effect of pain on daily living and also to study the effect of treatment. The occurrence of pain at night may have a bigger impact than pain during the day. Other aspects of pain like the various qualifications, locations, intensity, activities related are also of great importance to determine first before proceeding to interventional medical studies. Interviewing focused groups of patients and recruiting patients' information on the daily and social aspects where their SFN related complaints may interfere with is also be of great value to develop a pain-driven Rasch-built functional measure.

Also, we do not only want to be able to tell patients what is wrong, but also to be able to explain what to expect, as we can tell in other peripheral neuropathies like in 
Guillain-Barré syndrome (GBS) and chronic idiopathic axonal polyneuropathy(CIAP). ${ }^{27}$,

28 The natural course in SFN should be evaluated at a multi-level approach (at the pathology level: IENFD; impairment level: symptoms questionnaires; at the activity and participation level; functional questionnaires). All this will ultimately contribute to future clinical interventional studies.

It is also important to question what is important for patients. On the one hand, there may be no clinically relevant difference for a patient between for example an IENFD of 1 or 2 fibers/mm, although this might be informative to understand pathological/ impairments dynamics of an illness. On the other hand, pain is considered a better outcome determinant as has been addressed by the IMMPACT group. ${ }^{29}$

Quality of life is not just something to be documented. A multidisciplinary approach to treatment, seeking improvement in quality of life, is essential. Both drug treatment programs as rehabilitation programs need to be implemented and evaluated using appropriate quality of life instruments.

\section{Future studies}

Small fiber neuropathy is not really a disease entity but a syndrome, a set of signs and symptoms associated with several underlying diseases. As such, it is a starting point to search for a possible cause and possible solution. However, a common denominator may be genetic abnormalities since so many different problems (see introduction, table 1) give rise to a more or less similar syndrome. Currently, lots of efforts have been put by our group in unraveling the relationship between SFN and variants found in voltage gated sodium channels. ${ }^{1-3,30-33}$

The search for genetic abnormalities as the source of abnormal pain sensation is not only a possible answer to why different conditions lead to similar symptoms, but may also pave the way to development of targeted treatment.

Of the nine known isoforms of the human voltage gated sodium channel, five are expressed in the peripheral and autonomic nervous system and play a critical role of in the generation and conduction of action potentials. More specifically, their presence and function imply a key position in the nociceptive pathway, which make them a target for neuropathic pain treatment..$^{34}$

A large international multicenter multidisciplinary study (PROPANE) has been initiated, to provide translational research data on painful neuropathies. This project aims, amongst other goals: to explore genetic abnormalities in the different sodium channels by whole exome sequencing; to evaluate the effect of these variations on function and therefore mechanisms of neuropathic pain; to identify drug targets; to be able to identify patients at risk and explain the source of the nerve damage in different conditions. Both extensive studies in patients with painful neuropathies, and translational studies using zebrafish models will be performed. 
Besides this exciting research project, two other studies need to be mentioned. First, based on the mechanism of action of lacosamide (a novel anti-epileptic drug that dampens the over-activity of sodium channels by increasing slow inactivation), the painkilling effects of this drug in patients with proven genetic sodium channel abnormalities are currently investigated. Second, based on a bio-pschycho-social model, a tailored rehabilitation program with graded activity therapy will be offered to SFN patients and evaluated prospectively.

As usual, the conclusion of this study is that we are in need for more research. Luckily, there are enthusiastic and talented people striving to unravel SFN together, internationally! 


\section{REFERENCES}

1. Hoeijmakers JG, Faber CG, Lauria G, Merkies IS, Waxman SG. Small-fibre neuropathies-advances in diagnosis, pathophysiology and management. Nat Rev Neurol 2012;8:369-379.

2. Faber CG, Hoeijmakers JG, Ahn HS, et al. Gain of function Nanu1.7 mutations in idiopathic small fiber neuropathy. Ann Neurol 2012;71:26-39.

3. Huang J, Yang Y, Zhao P, et al. Small-fiber neuropathy Nav1.8 mutation shifts activation to hyperpolarized potentials and increases excitability of dorsal root ganglion neurons. J Neurosci 2013;33:14087-14097.

4. England JD, Gronseth GS, Franklin G, et al. Evaluation of distal symmetric polyneuropathy: the role of autonomic testing, nerve biopsy, and skin biopsy (an evidence-based review). Muscle Nerve 2009;39:106-115.

5. Hoitsma E, Reulen JP, de Baets M, Drent M, Spaans F, Faber CG. Small fiber neuropathy: a common and important clinical disorder. J Neurol Sci 2004;227:119-130.

6. Lauria G, Lombardi R. Skin biopsy: a new tool for diagnosing peripheral neuropathy. Bmj 2007:334:11591162.

7. Freeman R, Chase KP, Risk MR. Quantitative sensory testing cannot differentiate simulated sensory loss from sensory neuropathy. Neurology 2003;60:465-470.

8. Stewart JD, Low PA, Fealey RD. Distal small fiber neuropathy: results of tests of sweating and autonomic cardiovascular reflexes. Muscle Nerve 1992;15:661-665.

9. Tesfaye S, Boulton AJ, Dyck PJ, et al. Diabetic neuropathies: update on definitions, diagnostic criteria, estimation of severity, and treatments. Diabetes Care 2010;33:2285-2293.

10. Mano T, Iwase S, Toma S. Microneurography as a tool in clinical neurophysiology to investigate peripheral neural traffic in humans. Clin Neurophysiol 2006;117:2357-2384.

11. Lambert E, Hering D, Schlaich M, Lambert G. Advances in sympathetic nerve recording in humans. Front Physiol 2012;3:5.

12. Kleggetveit IP, Namer B, Schmidt R, et al. High spontaneous activity of C-nociceptors in painful polyneuropathy. Pain 2012;153:2040-2047.

13. Serra J, Collado A, Sola R, et al. Hyperexcitable C nociceptors in fibromyalgia. Ann Neurol 2014;75:196208.

14. Quattrini C, Tavakoli M, Jeziorska M, et al. Surrogate markers of small fiber damage in human diabetic neuropathy. Diabetes 2007;56:2148-2154.

15. Papanas N, Ziegler D. Corneal confocal microscopy: a new technique for early detection of diabetic neuropathy. Curr Diab Rep 2013;13:488-499.

16. Ziegler D, Papanas N, Zhivov A, et al. Early Detection of Nerve Fiber Loss by Corneal Confocal Microscopy and Skin Biopsy in Recently Diagnosed Type 2 Diabetes. Diabetes 2014.

17. Asch DA, Patton JP, Hershey JC. Knowing for the sake of knowing: the value of prognostic information. Medical decision making : an international journal of the Society for Medical Decision Making 1990;10:47-57.

18. Streiner D.L. GRN. Health measurement scales. A practical guide to their development and use, second ed. New York: Oxford University Press, 1998.

19. Merbitz C, Morris J, Grip JC. Ordinal scales and foundations of misinference. Arch Phys Med Rehabil 1989;70:308-312.

20. DeVellis RF. Classical test theory. Med Care 2006:44:S50-59.

21. Grimby G, Tennant A, Tesio L. The use of raw scores from ordinal scales: time to end malpractice? Journal of rehabilitation medicine 2012;44:97-98.

22. Tennant A, Conaghan PG. The Rasch measurement model in rheumatology: what is it and why use it? When should it be applied, and what should one look for in a Rasch paper? Arthritis Rheum 2007:57:1358-1362.

23. Rasch G. Probabilistic models for some intelligence and attainment tests. Chicago: University of Chicago Press, 1960.

24. van Nes SI, Vanhoutte EK, van Doorn PA, et al. Rasch-built Overall Disability Scale (R-ODS) for immunemediated peripheral neuropathies. Neurology 2011;76:337-345. 
25. Hermans MC, Faber CG, De Baets MH, de Die-Smulders CE, Merkies IS. Rasch-built myotonic dystrophy type 1 activity and participation scale (DM1-Activ). Neuromuscul Disord 2010;20:310-318.

26. Binda D, Vanhoutte EK, Cavaletti G, et al. Rasch-built Overall Disability Scale for patients with chemotherapy-induced peripheral neuropathy (CIPN-R-ODS). European journal of cancer 2013;49:29102918.

27. van den Berg B, Walgaard C, Drenthen J, Fokke C, Jacobs BC, van Doorn PA. Guillain-Barre syndrome: pathogenesis, diagnosis, treatment and prognosis. Nat Rev Neurol 2014;10:469-482.

28. Vrancken AF, Franssen H, Wokke JH, Teunissen LL, Notermans NC. Chronic idiopathic axonal polyneuropathy and successful aging of the peripheral nervous system in elderly people. Arch Neurol 2002;59:533-540.

29. Dworkin RH, Turk DC, McDermott MP, et al. Interpreting the clinical importance of group differences in chronic pain clinical trials: IMMPACT recommendations. Pain 2009;146:238-244.

30. Hoeijmakers JG, Merkies IS, Gerrits MM, Waxman SG, Faber CG. Genetic aspects of sodium channelopathy in small fiber neuropathy. Clinical genetics 2012;82:351-358.

31. Faber CG, Lauria G, Merkies IS, et al. Gain-of-function Nav1.8 mutations in painful neuropathy. Proc Natl Acad Sci U S A 2012;109:19444-19449.

32. Han C, Hoeijmakers JG, Ahn HS, et al. Nav1.7-related small fiber neuropathy: impaired slow-inactivation and DRG neuron hyperexcitability. Neurology 2012;78:1635-1643.

33. Huang J, Han C, Estacion M, et al. Gain-of-function mutations in sodium channel NaV1.9 in painful neuropathy. Brain 2014.

34. J.G.J. H. Small fiber neuropathy and sodium channels, a paradigm shift. Datawyse/Universitaire pers Maastricht: Maastricht University Medical Center, 2014. 
Chapter

8.2 

Chapter 9

\section{NEDERLANDSE SAMENVATTING}




\section{Chapter 9.1}

\section{SAMENVATTING EN ALGEMENE DISCUSSIE}

Dit proefschrift bevat een serie studies betreffende dunne vezel neuropathie (small fiber neuropathy, SFN). Hieronder wordt een samenvatting geschetst van hetgeen in deze thesis is gepresenteerd. Het potentieel belang alsook de eventuele implicaties voor toekomstig onderzoek wordt besproken.

\section{Inleiding}

Hoofdstuk 1 bevat een algemene inleiding, met zowel klinische informatie over SFN als de opzet van het proefschrift. Patiënten met SFN hebben spontane pijn en/ of autonome stoornissen door disfunctie van de A-delta en C vezels. Een combinatie van klachten, afwijkingen bij neurologisch-, neurofysiologisch- en pathologisch onderzoek wordt gebruikt om de diagnose te bevestigen. Er zijn vele aandoeningen geassocieerd met het optreden van SFN, van intoxicaties, metabole-, inflammatoireen neoplastische aandoeningen. Mutaties in spanningsafhankelijke natrium kanalen kunnen ook SFN veroorzaken. ${ }^{1-3}$

\section{Diagnosticeren van dunne vezel neuropathie}

Een gouden standaard voor de diagnose SFN ontbreekt, helaas. Veranderingen van dikke en dunne vezels kunnen pathologisch onderzocht worden in nervus suralis biopten, echter dit is een invasieve procedure en wordt over het algemeen alleen aanbevolen wanneer aan amyloïdose of vasculitis wordt gedacht. ${ }^{4}$ Diverse diagnostische testen zijn derhalve ontwikkeld om specifieke functies van het zenuwstelsel te onderzoeken (gevoelsdrempel testen, opgewekte potentialen, sudomotore en cardiovasculaire reflex testen). Echter, deze onderzoeken zijn vaak subjectief, moeilijk uit te voeren of hebben een beperkte sensitiviteit of specificiteit. ${ }^{5}$

De intra-epidermale zenuwvezeldichtheid (IENFD) van een huidbiopt wordt beschouwd als een objectieve diagnostische test voor SFN. Met een klein $3 \mathrm{~mm}$ stansbiopt kan de huid van een aangedaan, of gestandaardiseerd huidgebied (10 $\mathrm{cm}$ boven de laterale malleolus) onderzocht worden. Dit is een minimaal invasieve procedure, met weinig tot geen nadelige neveneffecten voor de patiënt. ${ }^{6}$ 
In hoofdstuk2.1 worden normaalwaarden voor intra-epidermalezenuwvezeldichtheid (intraepidermal nerve fiber density, IENFD) gepresenteerd. Allereerst werden data verzameld van gezonde Nederlandse en Italiaanse vrijwilligers. Er bleek sprake van een leeftijdsafhankelijke afname van IENFD, met lagere vezeldichtheden in mannen ten opzichte van vrouwen. Klinische toepasbaarheid werd getoond in een cohort sarcoïdose patiënten. Vervolgens werd een internationale collaboratie gevormd, tussen acht huidbiopt laboratoria in Europa, de VS en Azië. Dit resulteerde in wereldwijd toepasbare referentiewaarden en validatie van de bevindingen van ons instituut (hoofdstuk 2.2). Al met al is het gebruik van IENFD in de klinische praktijk mogelijk geworden door de (voor leeftijd en geslacht) gestratificeerde normaalwaarden die deze onderzoeken hebben opgeleverd.

Hoewel de afname techniek van een huidbiopt vrij simpel is en weinig invasief, zijn de handelingen erna (fixeren, kleuren, tellen) tijdsintensief en is dit niet in elk pathologisch laboratorium mogelijk. Bovendien is de sensitiviteit van IENFD vermoedelijk beperkt, vanwege de grote variatie in vezeldichtheid bij gezonde mensen. Daardoor is het meestal nodig de diagnose SFN te stellen door middel van een combinatie van IENFD en andere onderzoeken.

Hoofstuk 3.1 bevat een review over temperatuur drempel onderzoek (temperature threshold testing, TTT), een breder beschikbare, kwantitatieve, techniek, die vaak gebruikt wordt voor SFN evaluatie. Er is een grote variatie in gebruikte apparatuur, onderzochte modaliteiten, test methodologie en wijze van rapportage van uitkomsten. Er blijkt een gebrek aan internationale standaardisatie bij gebruik van TTT. Enkele praktische, op beschikbare literatuur gebaseerde, aanbevelingen zijn bijvoorbeeld: testen van warmte en koude sensatie drempels; gestratificeerde normaalwaarden gebruiken; gebruik van een reactietijd onafhankelijk test algoritme. De responsiviteit van TTT moet nog middels klinische studies onderzocht worden.

Middels onderzoeksgegevens van vele patiënten werd een uitgebreid, voor patiënt en onderzoeker belastend, TTT protocol geoptimaliseerd, met behoud van sensitiviteit en specificiteit (hoofdstuk 3.2).

Het beste resultaat werd verkregen door warmte en koude drempel te meten aan voeten en handen op een reactie tijd onafhankelijke wijze. Uiteraard dient een patiënt hierbij coöperatief en goed geconcentreerd te zijn, en is het resultaat subjectief waardoor simulatie niet volledig uitgesloten kan worden.?

In theorie worden deze beperkingen omzeild door het meten van door hitte-contact opgewekte cerebrale potentialen (contact heat evoked potentials, CHEPs), aangezien men niet verwacht dat de patiënt de uitkomst hiervan kan bepalen. Hoofdstuk 4 beschrijft het verkrijgen van CHEPs van een groot cohort gezonde Nederlanders. Latenties bleken gerelateerd aan lengte, amplitudes beïnvloed door geslacht. Er is een 
leeftijdsafhankelijke afname van amplitudes met geringe toename van latenties bij toenemende leeftijd. Er werden gestratificeerde normaalwaarden verkregen waarmee CHEP resultaten van patiënten geëvalueerd kunnen worden.

Helaas zijn er enkele praktische problemen verbonden aan CHEPs. In sommige gezonde mensen kon geen reactie opgewekt worden, eerder ook door anderen beschreven, hetgeen leidt tot een lage specificiteit. Het is mogelijk dat sommige "gezonde mensen" subklinische sensibele stoornissen hebben waardoor deze CHEP afwijkingen veroorzaakt worden. Daarnaast werd een duidelijkere respons gezien bij een hogere start temperatuur, echter een andere start temperatuur kan leiden tot andere latentie en mogelijk amplitude. Standaardisatie voor de klinische praktijk is dan ook belangrijk en moet verder onderzocht worden zodat gebruik van CHEPs en toegevoegde waarde hiervan bij de diagnostiek van SFN duidelijk wordt. Wij zagen in $74 \%$ van de patiënten met een verlaagde IENFD een afwijkende CHEP of TTT waarde, maar de relatie tussen CHEPS, TTT en IENFD dient verder opgehelderd te worden. Bovendien vinden patiënten CHEPs een pijnlijk onderzoek, erger dan bijvoorbeeld een huidbiopt, waardoor praktische toepasbaarheid mogelijk beperkt wordt.

\section{Klinische en klinimetrische aspecten van dunne vezel neuropathie}

Het bestaan van polyneuropathieën met (overwegend) dunne vezel betrokkenheid wordt reeds tientallen jaren onderkend, ${ }^{8}$ maar de aandacht gaat overwegend uit naar diabetische polyneuropathieën, vermoedelijk vanwege de grote aantallen aangedane patiënten. De afgelopen jaren is de interesse in en de zoektocht naar onderliggende oorzaken toegenomen, alsook de behoefte aan meer specifieke behandelingen. SFN is geen ongebruikelijke aandoening en zelfs als geen oorzaak of behandeling bestaat kunnen patiënten gebaat zijn bij een syndroomdiagnose. Het komt voor dat klachten jarenlang miskend worden (en bijvoorbeeld behandeld worden met cognitieve gedragstherapie) voordat duidelijk wordt dat SFN de oorzaak is.

Een schatting van de (minimale) incidentie en prevalentie wordt gepresenteerd in hoofdstuk 5, betreffende het verzorgingsgebied van het Maastricht Universitair Medisch Centrum. Hier is sprake van een incidentie van minimaal 12/100.000/jaar en prevalentie van 53/1000.000, aanzienlijke getallen, met name wanneer rekening wordt gehouden met het gegeven dat de werkelijke cijfers naar alle waarschijnlijkheid hoger uitvallen wanneer niet alleen naar verwezen patiënten gekeken wordt. Hopelijk neemt bewustwording van SFN en hieraan gerelateerde klachten toe in de toekomst.

Verbeterde herkenning van pijnlijke polyneuropathieën en autonome verschijnselen, als onderdeel van het brede spectrum van de polyneuropathieën, is belangrijk. Zowel voor patiënt als arts, aangezien dit de diagnostische en behandelstrategieën bepaalt en kan leiden tot gepaste uitleg omtrent de klachten. 
In hoofdstuk $\mathbf{6}$ worden pijn en autonome disfunctie middels vragenlijsten geëvalueerd in een groep sarcoïdose patiënten met en zonder mogelijke SFN. Autonome klachten werden gerapporteerd door alle patiënten, maar duidelijk meer in patiënten die werden verdacht van SFN (hetgeen voor de hand ligt, de verdenking SFN is gebaseerd op het anamnestisch bestaan van pijn en autonome disfunctie). Pijn werd eveneens door iedereen gerapporteerd, maar was ernstiger in patiënten met een verlaagde IENFD, en vooral bij de meer specifiek neuropathische pijntypes. Het gebruik van vragenlijsten kan de ernst van problemen verhelderen en mogelijk bijdragen aan de herkenning van SFN.

Hoofdstuk 7 onderzoekt de invloed van SFN op kwaliteit van leven. Patiënten met SFN ervaren een ernstige algehele reductie van kwaliteit van leven, pijn verklaart slechts een deel hiervan. In dit onderzoek, de SF-36, een generieke gezondheid gerelateerde kwaliteit van leven vragenlijst, werd gebruikt en vergeleken met gepubliceerde normaalwaarden voor de Nederlandse bevolking. De invloed van SFN is aanzienlijk, waarbij de scores vergelijkbaar zijn met die van patiënten die recent een hartinfarct hebben doorgemaakt. Pijn en autonome verschijnselen verklaarden 32\% van de afname in kwaliteit van leven. Dit impliceert dat andere aspecten, zoals verstoorde slaap, vermoeidheid en stemmingsstoornissen, ook van belang zullen zijn.

\section{Al met al kunnen we concluderen dat interesse in (patiënten met) SFN geen luxe maar noodzaak is.}




\section{Chapter 9.2}

\section{TOEKOMST PERSPECTIEVEN}

\section{Diagnosticeren van SFN}

Het gebrek aan een gouden standaard voor de diagnose SFN zal een uitdaging blijven. Hoewel clinici tegenwoordig elkaars criteria voor de definitie en diagnose overnemen, ${ }^{9}$ hebben TTT en IENFD als bevestigende onderzoeken beperkte sensitiviteit en specificiteit. Er is behoefte aan betrouwbare, valide en responsieve instrumenten om SFN te diagnosticeren.

Zenuwgeleidingsonderzoek is alleen bruikbaar om dikke vezel betrokkenheid te bepalen, in SFN is dit onderzoek per definitie normaal. Zoals eerder beschreven zijn er enkele beperkingen van TTT: het is subjectief, moeilijk te vergelijken tussen centra die verschillende protocollen gebruiken, betrouwbaarheid hangt af van testmethode en testlocatie, validiteit en responsiviteit zijn onbekend. Hoewel opgewekte potentialen, door laser of hitte-contact, een veelbelovend alternatief lijkt, is dit een pijnlijk en mogelijk lastig te implementeren onderzoek.

Microneurografie is een gespecialiseerde techniek die informatie levert over dunne sensibele en autonome vezels. Met een metalen micro-electrode, die in een perifere zenuw ingebracht wordt, kunnen afferente en efferente ontladingen worden geregistreerd. Veel onderzoek is gewijd aan sympathische microneurografie, ter beoordelingen van de neurale autonome regulatie. ${ }^{10,11}$ Afferente nociceptieve ontladingen van C-vezels kunnen ook geregistreerd worden, bij pijnlijke polyneuropathie zijn hyperexcitatie en sensitisatie van C-vezels aangetoond. ${ }^{12}$ Deze techniek kan inzicht bieden in zenuw (dis)functie en in chronische pijnsyndromen zoals fibromyalgie, waarbij hyperexcitatie en sensitisatie ook zijn aangetoond. ${ }^{13}$ Echter, deze techniek is niet wijdverbreid en weinig artsen zijn in staat deze registraties uit te voeren. Het is bovendien een tijdrovend onderzoek en tot nog toe zijn weinig patiënten onderzocht met het oog op SFN en vooralsnog zijn er geen gestratificeerde normaalwaarden. Momenteel is de waarde hiervan voor het diagnosticeren van SFN nog onzeker, maar het is voor wetenschappelijk onderzoek een interessante techniek. Hoewel IENFD bepaling middels een huidbiopt betrouwbaar en valide is gebleken, moet responsiviteit nog onderzocht worden om te kunnen beoordelen of dit als 
uitkomstmaat kan dienen in klinische studies. Het is verder (minimaal) invasief met beperkte beschikbaarheid. Confocale microscopie van de cornea zou een geschikt alternatief kunnen zijn. Na toedienen van lokale verdoving wordt een microscooplens op de cornea gepositioneerd. Met een digitale camera wordt een reeks foto's vervaardigd die nadien geanalyseerd kunnen worden. Een speciale microscoop die geschikt is voor in vivo oogonderzoek is noodzakelijk, maar een dergelijke microscoop heeft verschillende toepassingen in de oftalmologische praktijk. Op non-invasieve wijze kan hiermee zenuwvezeldichtheid van de cornea onderzocht worden. Het lijkt erop dat corneale vezeldichtheid net zo informatief is als IENFD. ${ }^{14-16}$

Vermoedelijk is een combinatie van onderzoeken, wellicht stapsgewijs uitgevoerd, uiteindelijk de beste methode om de diagnose SFN te bevestigen. Er zijn plannen deze aspecten rondom de diagnostische aanpak van SFN in een internationale bijeenkomst aan te kaarten, in de hoop tot een consensus te komen.

\section{Klinische en klinimetrische aspecten van dunne vezel neuropathie}

Zoals eerder aangegeven, zelfs als (nog) geen behandeling voorhanden is, kunnen patiënten gebaat zijn bij tijdige (h)erkenning van de diagnose SFN. ${ }^{17}$ Daarom zijn valide en betrouwbare screeningsinstrumenten en vragenlijsten nodig om mogelijke SFN patiënten te identificeren. Deze middelen dienen bij voorkeur responsief te zijn indien ze gebruikt gaan worden bij klinische studies. ${ }^{18}$ De meeste vragenlijsten die bestaan voor patiënten met een polyneuropathie zijn ordinale multi-item composiet schalen, die ondanks algemeen bekende tekortkomingen nog steeds gebruikt worden. ${ }^{19-21}$

Ordinale schalen leveren in essentie descriptieve data zonder numerieke waarde. Moderne middelen zoals de Rasch methodologie worden toenemend gebruikt om vragenlijsten te onderzoeken, verbeteren en creeëren. ${ }^{22,23 ~ " H e t ~ R a s c h ~ m o d e l ~ g e e f t ~ a a n ~}$ dat de kans dat een patiënt een vraag correct kan beantwoorden of een taak kan uitvoeren is een logistische functie van de moeilijkheid van de taak en de mogelijkheid van de patiënt die te volbrengen". Voor verschillende neuromusculaire ziekten, zijn zo specifieke uitkomstmaten vervaardigd.24-26 Op diezelfde manier zal een op Raschgebaseerde beperkingen vragenlijst worden geconstrueerd, specifiek voor SFN, die aan de diverse klinimetrische eisen voldoet.

Differentiatie van pijn ervaringen kunnen verder gedifferentieerd worden om de effecten van pijn op het dagelijks leven te evalueren en de effecten van therapie te bestuderen. Het optreden van nachtelijke pijn heeft wellicht een grotere impact dan pijn overdag. Andere aspecten zoals de verschillende soorten pijn, locaties, intensiteit, relatie tot activiteiten, zijn eveneens van belang om vast te stellen alvorens over te gaan tot medicatie studies. Om een adequate pijn vragenlijst volgens Rasch methodologie te ontwikkelen, kunnen focus groep interviews en het vergaren van informatie over hoe SFN gerelateerde problemen interfereren met het dagelijkse en sociale leven van patiënten, van groot belang zijn. 
Niet alleen willen we patiënten kunnen vertellen wat er met ze aan de hand is, maar ook wat ze kunnen verwachten, zoals mogelijk is voor andere polyneuropathieën zoals Guillain-Barré syndroom (GBS) en chronische idiopathische axonale polyneuropathie (CIAP). ${ }^{27,28}$ Het natuurlijke beloop van SFN moet op meerdere niveaus ontrafeld worden (pathologie: IENFD; beperkingen: klachten vragenlijsten; activiteiten en participatie: functionele vragenlijsten). Dit alles zal uiteindelijk bijdragen aan toekomstige interventie studies.

Het blijft belangrijk je af te vragen wat voor patiënten belangrijk is. Zo kan er klinisch geen relevant verschil zijn voor een patiënt tussen bijvoorbeeld een IENFD van 1 of 2 vezels/mm, hoewel dit kan bijdragen aan het begrip van de dynamiek tussen pathologie en beperking. Pijn wordt daarentegen beschouwd als een geschikte uitkomstmaat, zoals ook vastgesteld door de IMMPACT groep. ${ }^{29}$ Kwaliteit van leven is niet iets om alleen maar vast te leggen. Een multidisciplinaire aanpak die tot doel heeft kwaliteit van leven te bevorderen is essentieel. Zowel medicamenteuze interventies als revalidatie trajecten moeten ingezet worden en geëvalueerd worden met geschikte meetinstrumenten ter evaluatie van kwaliteit van leven.

\section{Blik in de toekomst}

Dunne vezel neuropathie is in essentie geen ziekte maar een syndroomdiagnose, een combinatie van klachten en afwijkingen met diverse onderliggende aandoeningen. Als zodanig is het beginpunt voor een zoektocht naar mogelijke oorzaken en oplossingen. Echter, een genetische afwijking zou een gemeenschappelijke factor kunnen zijn, aangezien zoveel uiteenlopende aandoeningen (zie Tabel 1, introductie) min of meer hetzelfde syndroom veroorzaken.

Op dit moment wordt binnen onze onderzoeksgroep veel aandacht besteed aan het ontrafelen van de relatie tussen SFN en varianten in spanningsafhankelijke natrium kanalen. ${ }^{1-3,30-33}$ Deze zoektocht naar genetische afwijkingen als oorzaak van afwijkende pijnsensatie leidt wellicht niet alleen naar een antwoord waarom deze verschillende aandoeningen tot dezelfde symptomen lijden, maar ook tot ontwikkeling van een gerichte behandeling.

Van de negen bekende isovormen van het humane spanningsafhankelijke natriumkanaal komen er vijf voor in het perifere sensibele of autonome zenuwstelsel. Deze kanalen spelen een belangrijke rol bij het ontstaan en voort geleiden van actie potentialen. Hun aanwezigheid en functioneren doen een sleutelpositie vermoeden in nociceptie, waardoor ze een potentieel aangrijpingspunt zijn voor behandeling van neuropathische pijn. ${ }^{34}$

Een grote internationale multicenter multidisciplinaire studie (PROPANE) is opgezet om translationeel onderzoek te verrichten naar pijnlijke polyneuropathieën. Dit project heeft onder andere tot doel: genetische afwijkingen in de diverse natriumkanalen te exploreren middels "whole exome sequencing"; de effecten van deze afwijkingen op 
functioneren te evalueren, en daarmee het mechanisme van neuropathische pijn; aangrijpingspunten voor medicatie te identificeren; risico patiënten te herkennen en de oorzaak van zenuwschade in verschillende condities te verklaren. Hiertoe zullen patiënten met pijnlijke polyneuropathie worden onderzocht, maar ook zal translationeel onderzoek plaatvinden met behulp van zebravis modellen.

Behalve dit enorme onderzoeksproject zijn er nog andere studies die genoemd dienen te worden. Ten eerste wordt, gebaseerd op het werkingsmechanisme van lacosamide (een nieuw anti-epilepticum dat trage inactivatie van natriumkanalen doet toenemen en daardoor overactiviteit dempt), het pijnstillende effect van dit middel onderzocht bij patiënten met een bewezen genetische natrium kanalopathie. Ten tweede zal een revalidatieprogramma, gebaseerd op een bio-psycho-sociaal model, aangeboden worden aan een groep SFN patiënten en prospectief geëvalueerd worden.

Zoals gebruikelijk is de conclusie van dit proefschrift dat meer onderzoek nodig is. Gelukkig zijn er internationaal enthousiaste en getalenteerde mensen samen bezig de geheimen van dunne vezel neuropathie te ontrafelen! 


\section{REFERENTIES}

1. Hoeijmakers JG, Faber CG, Lauria G, Merkies IS, Waxman SG. Small-fibre neuropathies-advances in diagnosis, pathophysiology and management. Nat Rev Neurol 2012;8:369-379.

2. Faber CG, Hoeijmakers JG, Ahn HS, et al. Gain of function Nanu1.7 mutations in idiopathic small fiber neuropathy. Ann Neurol 2012;71:26-39.

3. Huang J, Yang Y, Zhao P, et al. Small-fiber neuropathy Nav1.8 mutation shifts activation to hyperpolarized potentials and increases excitability of dorsal root ganglion neurons. J Neurosci 2013;33:14087-14097.

4. England JD, Gronseth GS, Franklin G, et al. Evaluation of distal symmetric polyneuropathy: the role of autonomic testing, nerve biopsy, and skin biopsy (an evidence-based review). Muscle Nerve 2009;39:106-115.

5. Hoitsma E, Reulen JP, de Baets M, Drent M, Spaans F, Faber CG. Small fiber neuropathy: a common and important clinical disorder. J Neurol Sci 2004;227:119-130.

6. Lauria G, Lombardi R. Skin biopsy: a new tool for diagnosing peripheral neuropathy. Bmj 2007;334:11591162.

7. Freeman R, Chase KP, Risk MR. Quantitative sensory testing cannot differentiate simulated sensory loss from sensory neuropathy. Neurology 2003;60:465-470.

8. Stewart JD, Low PA, Fealey RD. Distal small fiber neuropathy: results of tests of sweating and autonomic cardiovascular reflexes. Muscle Nerve 1992;15: 661-665.

9. Tesfaye S, Boulton AJ, Dyck PJ, et al. Diabetic neuropathies: update on definitions, diagnostic criteria, estimation of severity, and treatments. Diabetes Care 2010;33:2285-2293.

10. Mano T, Iwase S, Toma S. Microneurography as a tool in clinical neurophysiology to investigate peripheral neural traffic in humans. Clin Neurophysiol 2006;117:2357-2384.

11. Lambert E, Hering D, Schlaich M, Lambert G. Advances in sympathetic nerve recording in humans. Front Physiol 2012;3:5.

12. Kleggetveit IP, Namer B, Schmidt R, et al. High spontaneous activity of C-nociceptors in painful polyneuropathy. Pain 2012;153:2040-2047.

13. Serra J, Collado A, Sola R, et al. Hyperexcitable C nociceptors in fibromyalgia. Ann Neurol 2014;75:196208.

14. Quattrini C, Tavakoli M, Jeziorska M, et al. Surrogate markers of small fiber damage in human diabetic neuropathy. Diabetes 2007;56:2148-2154.

15. Papanas N, Ziegler D. Corneal confocal microscopy: a new technique for early detection of diabetic neuropathy. Curr Diab Rep 2013;13:488-499.

16. Ziegler D, Papanas N, Zhivov A, et al. Early Detection of Nerve Fiber Loss by Corneal Confocal Microscopy and Skin Biopsy in Recently Diagnosed Type 2 Diabetes. Diabetes 2014.

17. Asch DA, Patton JP, Hershey JC. Knowing for the sake of knowing: the value of prognostic information. Medical decision making : an international journal of the Society for Medical Decision Making 1990;10:47-57.

18. Streiner D.L. GRN. Health measurement scales. A practical guide to their development and use, second ed. New York: Oxford University Press, 1998.

19. Merbitz C, Morris J, Grip JC. Ordinal scales and foundations of misinference. Arch Phys Med Rehabil 1989;70:308-312.

20. DeVellis RF. Classical test theory. Med Care 2006;44:S50-59.

21. Grimby G, Tennant A, Tesio L. The use of raw scores from ordinal scales: time to end malpractice? Journal of rehabilitation medicine 2012;44:97-98.

22. Tennant A, Conaghan PG. The Rasch measurement model in rheumatology: what is it and why use it? When should it be applied, and what should one look for in a Rasch paper? Arthritis Rheum 2007;57:1358-1362.

23. Rasch G. Probabilistic models for some intelligence and attainment tests. Chicago: University of Chicago Press, 1960.

24. van Nes SI, Vanhoutte EK, van Doorn PA, et al. Rasch-built Overall Disability Scale (R-ODS) for immunemediated peripheral neuropathies. Neurology 2011;76:337-345. 
25. Hermans MC, Faber CG, De Baets MH, de Die-Smulders CE, Merkies IS. Rasch-built myotonic dystrophy type 1 activity and participation scale (DM1-Activ). Neuromuscul Disord 2010;20:310-318.

26. Binda D, Vanhoutte EK, Cavaletti G, et al. Rasch-built Overall Disability Scale for patients with chemotherapy-induced peripheral neuropathy (CIPN-R-ODS). European journal of cancer 2013;49:29102918.

27. van den Berg B, Walgaard C, Drenthen J, Fokke C, Jacobs BC, van Doorn PA. Guillain-Barre syndrome: pathogenesis, diagnosis, treatment and prognosis. Nat Rev Neurol 2014;10:469-482.

28. Vrancken AF, Franssen $H$, Wokke JH, Teunissen LL, Notermans NC. Chronic idiopathic axonal polyneuropathy and successful aging of the peripheral nervous system in elderly people. Arch Neurol 2002;59:533-540.

29. Dworkin $\mathrm{RH}$, Turk DC, McDermott MP, et al. Interpreting the clinical importance of group differences in chronic pain clinical trials: IMMPACT recommendations. Pain 2009;146:238-244.

30. Hoeijmakers JG, Merkies IS, Gerrits MM, Waxman SG, Faber CG. Genetic aspects of sodium channelopathy in small fiber neuropathy. Clinical genetics 2012;82:351-358.

31. Faber CG, Lauria G, Merkies IS, et al. Gain-of-function Nav1.8 mutations in painful neuropathy. Proc Natl Acad Sci U S A 2012;109:19444-19449.

32. Han C, Hoeijmakers JG, Ahn HS, et al. Nav1.7-related small fiber neuropathy: impaired slow-inactivation and DRG neuron hyperexcitability. Neurology 2012;78:1635-1643.

33. Huang J, Han C, Estacion M, et al. Gain-of-function mutations in sodium channel NaV1.9 in painful neuropathy. Brain 2014

34. J.G.J. H. Small fiber neuropathy and sodium channels, a paradigm shift. Datawyse/Universitaire pers Maastricht: Maastricht University Medical Center, 2014. 


\section{Chapter 10}

\section{VALORISATION}

\section{Introduction}

Science serves a purpose, even when not immediately apparent. As researchers continuously question and examine current knowledge of every part of existence, this knowledge will expand and ultimately have impact on daily practice/life. Who would have thought Nikola Tesla's at the time "crazy experiments" would lead to the wireless radio, indoor lighting and electroshock therapy of today?

However, society has a right to ask anyone that wants support (either financial or otherwise) from that society, to justify that support. In the next chapter, the results of this thesis will be looked at from a more general point of view than just a medical one.

\section{Valorization}

Valorization means "determining value". More specifically in the context of research: "translation of knowledge into a (commercial) product, service or process".

How does this apply to the research presented in this thesis? We have presented new insight into the diagnosis of small fiber neuropathy (SFN), showed minimum incidence and prevalence of SFN in a defined population and shown several clinical characteristics of SFN and implications on patients' quality of life. Some more general implications of the results have been touched upon in previous chapters, but will be discussed in more detail in this chapter.

\section{Diagnosis of small fiber neuropathy}

The possibilities and limitations of several diagnostic techniques for SFN have been presented. By doing so, guidance can be created for clinicians, but also for policymakers, to determine what tests are of value in the diagnostic procedure. For example, standard neurological examination, nerve conduction studies (to examine other forms of polyneuropathy), and temperature threshold testing (using the presented optimized protocol) should be considered first step investigations in patients with possible (small fiber) neuropathy. This may lead to recognition of SFN and a directed search for underlying illnesses in a substantial amount of patients. Second, for those in whom a diagnosis is still elusive, determination of corneal or intra-epidermal nerve 
fiber density can be performed in a specialized center, where also other advanced techniques such as contact heat evoked potentials may be applied. Also, the decision to search more extensively for an etiological factor could be based on the results thereof. Microneurography might presently be reserved for research purposes only.

Following such a stepwise approach might not only lead to better diagnosis of patients with appropriate tests ("products") but may also lead to better recognition how to organize care for these patients ("services and process").

Also, the ongoing research into channelopathies is an important sequel, that may reveal the basis for pain and possibly provide targeted treatment, in a wider array of polyneuropathies.

\section{Clinical and clinimetric aspects of small fiber neuropathy}

With the presented minimum prevalence and incidence for SFN, better insight into the extent of the problem has been sketched. The socio-economic burden of small fiber neuropathy is better appreciated, and puts costs of research and interventions in perspective. Also, this improves the possibility to evaluate interventions on a population scale. A prevention program ("services and process") for diabetes mellitus (DM) may for example be evaluated also by incidence of SFN, as both glucose intolerance and DM are important associated diseases.

The delineation of symptoms of SFN and the evaluation of screening tools such as the questionnaires used in this thesis (see appendix) improves recognition of SFN. We hope earlier that appropriate diagnostic workup is initiated sooner with increasing awareness, possibly with the help of such questionnaires. As the impact on quality of life is substantial, this could be of importance for patients. The benefit of finding more appropriate treatment strategies are apparent when impact is appreciated correctly.

Not only patients and care workers are helped by better understanding of the extent of complaints and the impact on quality of life. Again, socio-economic burden is high in diseases with such a large impact on daily living and relevant outcome measures ("products") help improve evaluation of care both on an individual as on group level ("service and process").

It is equally important to realize what further research should focus on. Fortunately, a current large scale international study is dedicated to illuminate fundamental processes in SFN!

\section{Conclusion}

In this chapter, an effort has been made to put the thesis in a broader perspective. We think the steps made in this thesis lead beyond the doorway of the doctors' office. Though they may be small steps, they lead us forward. 


\section{Chapter}





\section{Dankwoord}

Mij is ooit verteld dat het dankwoord één van de (zo niet hèt) best gelezen hoofdstukken is van een proefschrift. Vast niet omdat het zo'n origineel stukje is, geen hoofdstuk dat zo inwisselbaar is als het dankwoord. Al variëren natuurlijk de namen van proefschrift tot proefschrift. Misschien omdat iedereen even wil controleren of hij/zij genoemd wordt? Wil eenieder vereeuwigd worden in een drukwerk? Dan zal dit hoofdstuk vermoedelijk een heleboel mensen teleurstellen...

Allereerst gaat mijn dank uit naar alle patiënten en vrijwilligers die bereid zijn geweest zich te onderwerpen aan diverse vragenlijsten, onderzoeken en ingrepen. Zonder hen was er niets (geen reden, geen resultaten, geen motivatie) geweest om artikelen over te schijven, laat staan een proefschrift samen te stellen. Dus duizendmaal dank!

Ooit nam ik mij voor, dat als ik het onzalige idee op zou vatten te promoveren, het wel over iets zinnigs moest gaan. Zou u menen dat dat gelukt is, dan is dat geheel te danken aan Karin en Ingemar. Zonder hun ideeën, navorsingen, protocollen, analyses, aanmoedigingen, correcties en ga zo maar door, was er niets van terecht gekomen. Ze zijn voor mij grote voorbeelden (altijd positief en enthousiast) en tevens schrikbeelden (nooit slapende "dag en nachtwerkers"). Bij deze wil ik jullie dus danken voor al jullie harde werk en de moeite die jullie hebben genomen mij hierdoorheen te slepen! Ook hierbij veel dank aan de leden van de corona, het kritisch doorlezen van een proefschrift is een hele klus!

Zonder Pieter was ik overigens nooit bij Karin en Ingemar terecht gekomen om onderzoek te gaan doen, dus naar mijn idee een terechte vermelding alhier. Dit onderzoek kon verricht worden mede dankzij Elske, die dunne vezel neuropathie bij sarcoïdose op de kaart zette. Ook zouden zonder Marjolein en haar ILD team (met Gé, Gerry, Sita, Anita) vast nooit zoveel patiënten hiervoor te vinden zijn geweest.

Crucial to this thesis was the work on intraepidermal nerve fiber density. This was made possible by Giuseppe, whose work in this field was and is essential. I want to thank you for your energetic and inspiring help in this project. Of course many thanks also to Rafaela, Gracia, and Paola, who have taught me much, and always with a smile. 
Ook heb ik uiteraard veel te danken aan Jan, met wie ik eindeloos veel "huidjes" heb gekleurd, Benoit en ook de andere laboranten op de histologie en immunologie, die mijn aanwezigheid en gebruik van ruimte, "kleurstoffen" en apparatuur volledig aanvaardden. Ilse en Aline dank ik voor hun inzet om verwerking en beoordeling van de huidbiopten zo secuur mogelijk over te nemen. Natuurlijk ook veel dank aan Marc, zonder een laptop, werkplek en microscoop was er heel wat minder uit mijn handen gekomen.

Om nog even een cliché erin te gooien, onderzoek doe je niet alleen. Maar dat niet alleen, je bent meestal niet als enige onderzoek aan het doen. Zo had ik gelukkig Mieke, om gezamenlijke frustraties mee te bespreken tijdens de lunch. Na een eerste korte kennismaking in het CZE kwamen we elkaar weer tegen als AIO in het MUMC, waar we vrijwel tegelijk begonnen. Ik ben blij dat ik jouw rust en vertrouwen in goede afloop als voorbeeld heb gehad, dank dat je mijn paranimf wil zijn! Ook als AIOS kon ik altijd op je rekenen als fijne collega, klankbord en kamergenoot tijdens nascholingen. Brigitte probeert voor iedereen een zonnetje te zijn en de dag wat extra warmte te geven. Geen wonder dat mijn (oud)oom Ad haar als "aangenomen kleindochter" had omarmd. En wat een geluk dat we elkaar in Rotterdam eindelijk leerden kennen, om vervolgens allebei in Maastricht te belanden. Dank voor je steun en zonnestralen, ik hoop dat ik over een poosje jouw paranimf kan zijn!

Ik word ook altijd vrolijk als ik Sonja weer eens zie. Nadat we tegelijk waren gestart "op Noord", liep ons pad uiteen en toch weer samen, aangezien we door het onderzoek weer wat bijeengebracht werden. Nu ben ik wel eens nukkig, maar gelukkig, jij wees me op een remedie, al heb ik niet altijd een geschikt potlood bij de hand! Janneke pluist met zoveel plezier het pijnmechanismen uit, die heeft alleen af en toe een Tony Chocelonely nodig om op gang te blijven. Dank je dat jij, waar ik ben blijven hangen in onderzoeken, hard vooruit bent gegaan naar oorzaken. Ook eeuwig energiek is Els, alles wordt rap aan Rasch analyses onderworpen. Dankzij jou zullen patiënten straks alleen nog geplaagd worden met nuttige vragenlijsten. En natuurlijk dank aan Martine en Anne voor de geleverde inzet voor het onderzoek. Veel dank aan de mensen die verschillende onderdelen van het onderzoek op diverse wijzen voortzetten (Bianca, Tim, Carla, Charlotte) waardoor het kans heeft ook in de praktijk een plek te krijgen.

Wat die vragenlijsten betreft, die hebben niet alleen de patiënten bij het invullen, maar ook Kris bij het voorbereiden veel tijd gekost. Zo mooi als jij ze maakt zijn ze niet vaak, dank!

Ook andere zaken hebben de nodige tijd en energie gekost, soms zonder dat het ergens toe leidde ("dubbel piek stimulatie..."), soms juist met zeer bruikbare resultaten tot gevolg. Hierbij is de hulp van onder andere Vera, Jos, de KNF laboranten in 
Maastricht en Spaarne onontbeerlijk geweest, waarbij ik vooral van Ineke meermaals misbruik heb mogen maken, dank daarvoor.

Nu was ik niet altijd met "mijn eigen" onderzoek bezig, maar raakte ik ook betrokken bij diverse andere projecten, hetgeen zeer leerzaam was en waarbij ik met plezier heb samen gewerkt met onder andere Marieke, Rachel en Danielle.

Dat ik na 3 jaar onderzoek niet klaar was, betekende dat ik tijdens de opleidingstijd die daarop volgde nog veel te doen had, maar ondertussen natuurlijk ook veel te leren had. Ik wil Robert, al mijn andere opleiders, Kitty, secretariaat, polidames en collega assistenten danken voor de leerzame lessen, de gegeven ruimte (in tijd, plaats, ontwikkeling) en ondersteuning, maar vooral dank voor de facilitatie van zowel onderzoek als differentiatiestages. Opdat ik een "goed product" ben geworden!

Mijn familie en vrienden wil ik danken voor het vergoelijken van mijn infrequente langskomen, het frequent aanhoren van gezeur, en het betrachten van een mix van voldoende interesse, opbeurende woorden en rustgevende onverschilligheid. Fijn dat ik nog altijd jullie dochter/zus/vriendin mag zijn!

Robert mijn lief, dank dat je alle frustraties, vanwege software sores of vileine reviewers, in stilte hebt verdragen. Dank dat je ondanks mijn gestamp en gegooi met deuren niet zelf de deur uit bent gestevend. Dank dat je, al die keren dat ik op het punt stond de computer door het raam te smijten, mij er niet door hebt gekieperd. Ik hou van je. 


\section{Curriculum Vitae}

Mayienne Bakkers was born on the 28th of June 1978 in Kilgoris, Narok district, Kenya. She graduated in 1996 from the "Stedelijk Gymnasium Arnhem". After obtaining a propaedeutic diploma in mechanical engineering at the "HAN university of applied sciences" in Arnhem, she started her medical training at Maastricht University in 1997, graduating (cum laude) in 2003. Subsequently she started working as a clinical resident in neurology, first at the Catharina Hospital Eindhoven, in 2005 at the Erasmus Medical Center Rotterdam. In 2006 she started on the research project regarding small fiber neuropathy at the Maastricht University Medical Center. Her neurology training started in 2009 and was completed at the start of 2015. Recently she started work as a neurologist at Sint Jans Gasthuis Weert.

\section{LIST OF PUBLICATIONS}

Bakkers M, Faber CG, Reulen JPH, Hoeijmakers JG, Vanhoutte EK, Merkies IS. Optimizing Temperature threshold testing in small fiber neuropathy. Muscle Nerve.2014; Oct 7 epub ahead of print.

Lagerberg V, Bakkers M, Bouwhuis A, Hoeijmakers JG, Smit AM, van den berg SJ, Hordijk-de Boer I, Brouwer van der Lee MD, Kranendonk D, Reulen JP, Faber CG, Merkies IS. Contact heat evoked potentials: normal values and us in small fiber neuropathy. Muscle Nerve 2014; Sep 26 epub ahead of print.

Peters MJ, Bakkers M, Merkies ISJ, Hoeijmakers JG, van Raak EP, Faber CG. Incidence and prevalence of small fiber neuropathy: A survey in the Netherlands. Neurology 2013; 81:1356-1360

Bakkers M, Faber CG, Hoeijmakers JGJ, Lauria G, Merkies IS. Small fibers, large impact: quality of life in small fiber neuropathy, Muscle Nerve.2014; 49(3):329-336.

Binda D, Vanhoutte EK, Cavaletti G, Cornblath DR, Postma TJ, Frigeni B, Alberti P, Bruna J, Velasco R, Argyriou AA, Kalofonos HP, Psimaras D, Ricard D, Pace A, Galiè E, Briani C, Dalla Torre C, Lalisang RI, Boogerd W, Brandsma D, Koeppen S, Hense J, Storey D, Kerrigan S, Schenone A, Fabbri S, Rossi E, Valsecchi MG, Faber CG, Merkies IS; CI-PeriNomS study group, Galimberti S, Lanzani F, Mattavelli L, Piatti ML, Bidoli P, Cazzaniga M, Cortinovis D, Lucchetta M, Campagnolo M, Bakkers M, Brouwer B, Boogerd W, Grant R, Reni L, Piras B, Pessino A, Padua L, Granata G, Leandri M, Ghignotti I, Plasmati R, Pastorelli F, Heimans JJ, Eurelings M, Meijer RJ, Grisold W, Lindeck Pozza E, Mazzeo A, Toscano A, Russo M, Tomasello C, Altavilla G, Penas Prado M, Dominguez Gonzalez C, Dorsey SG. Rasch-built Overall Disability Scale for patients with chemotherapy induced peripheral neuropathy (COPN-R-ODS).Eur J Cancer 2013; 49(13):2910-2918. 
Bakkers M, Faber CG, Peters MJH, Reulen JP, Franssen H, Fischer TZ, Merkies IS. Temperature threshold testing: a systematic review. J Peripher Nerv Syst.2013; 18:7-18.

Pluijms WA, Slangen R, Bakkers M, Faber CG, Merkies IS, Kessels AG, Dirksen CD, Joosten EA, Reulen JP, van Dongen RT, Schaper NC, van Kleef M. Pain relief and quality-of-life improvement after spinal cord stimulation in painful diabetic polyneuropathy: a pilot study. Br J Anaesth. 2012; 109(4):623-629. Erratum in: Br J Anaesth. 2012; 109(5):837.

Biegstraaten M, Hollak CE, Bakkers M, Faber CG, Aerts JM, van Schaik IN. Small fiber neuropathy in Fabry disease.Mol Genet Metab. 2012; 106(2):135-141.

Hoeijmakers JG, Bakkers M, Blom EW, Drenth JP, Merkies IS, Faber CG. Small fibre neuropathy: knowledge is power. Ned Tijdschr Geneeskd. 2012; 156(7):A4224.

Peters MJ, van Nes SI, Vanhoutte EK, Bakkers M, van Doorn PA, Merkies IS, Faber CG; PeriNomS Study group. Revised normative values for the Jamar dynamometer. JPNS 2011; 16(1):47-50.

Hermans MC, Faber CG, Vanhoutte EK, Bakkers M, De Baets MH, de Die-Smulders CE, Merkies IS. Peripheral neuropathy in myotonic dystrophy type 1. JPNS 2011; 16(1):24-29.

van Nes SI, Vanhoutte EK, van Doorn PA, Hermans M, Bakkers M, Kuitwaard K, Faber CG, Merkies IS. Rasch-built Overall Disability Scale (R-ODS) for immune-mediated peripheral neuropathies. Neurology 2011; 76(4):337-345.

Lauria G, Bakkers M, Schmitz C, Lombardi R, Penza P, Devigili G, Smith AG, Hsieh ST, Mellgren SI, Umapathi T, Ziegler D, Faber CG, Merkies IS. Intraepidermal nerve fiber density at the distal leg: a worldwide normative reference study. J Peripher Nerv Syst. 2010; 15(3):202-207.

Bakkers M, Faber CG, Drent M, Hermans MC, van Nes SI, Lauria G, De Baets M, Merkies IS. Pain and autonomic dysfunction in patients with sarcoidosis and small fibre neuropathy. J Neurol. 2010; 257(12):2086-2090.

Bakkers M, Merkies IS, Lauria G, Devigili G, Penza P, Lombardi R, Hermans MC, van Nes SI, De Baets M, Faber CG. Intraepidermal nerve fiber density and its application in sarcoidosis. Neurology 2009; 73:1142-1148.

van Nes SI, Faber CG, Hamers RM, Harschnitz O, Bakkers M, Hermans MC, Meijer RJ, van Doorn PA, Merkies IS; PeriNomS Study Group. Revising two-point discrimination assessment in normal aging and in patients with polyneuropathies. J Neurol Neurosurg Psychiatry. 2008; 79(7):832-834. 


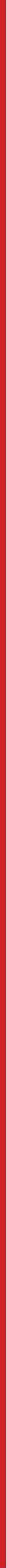

